\title{
STATUS OF THE FLORA AND FAUNA ON THE NEVADA TEST SITE, 1988
}

\section{Results of Continuing Basic Environmental Monitoring January through December 1988}

\author{
Compllod By \\ Richard B. Huntor \\ June 1992 \\ Work Performed Under Contract No. DE-AC08-89NV10630
}

Prepared for the

Unlted States Department of Energy

Nevada Field Otfice

Propared by

Reynolds Electrical \& Englnearing Co., Inc.

Post Offle Box 98521

Las Vogas, NV $89193-8521$ 


\begin{abstract}
In 1987 the U.S. Department of Energy (DOE) initiated a program to monitor the health of the Nevada Test Site (NTS) plants and animals in support of the National Environmental Protection Act. The program, part of DOE's Basic Environmental Compliance and Monitoring Program (BECAMP), monitors perennial and ephemeral plants, the more cornmon species of rodents and lizards, and the horses, deer, raptors and other large animals on the NTS. This is a report of data collected on these flora and fauna for the year 1988, the second year of monitoring.
\end{abstract}




$$
\text { -iv - }
$$$$
\text { - }
$$ 


\section{TABLE OF CONTENTS}

\section{Section}

Page

EXECUTIVE SUMMARY $\ldots \ldots \ldots \ldots \ldots \ldots \ldots \ldots \ldots \ldots \ldots \ldots \ldots$

1 EXTENT OF LAND DISTURBANCE ON THE NEVADA TEST SITE $\ldots \ldots \ldots \ldots \ldots$

2 STATUS OF DESERT EPHEMERALS ON THE NEVADA TEST SITE IN $1988 \ldots \ldots$

INTRODUCTION $\ldots \ldots \ldots \ldots \ldots \ldots \ldots \ldots \ldots \ldots \ldots \ldots \ldots \ldots$

STUDY SITES AND METHODS $\ldots \ldots \ldots \ldots \ldots \ldots \ldots \ldots \ldots \ldots \ldots$

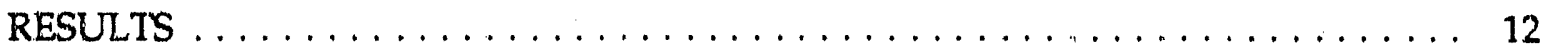

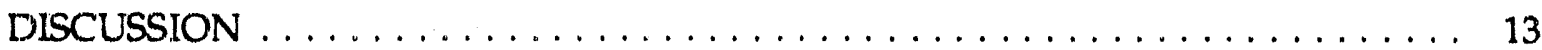

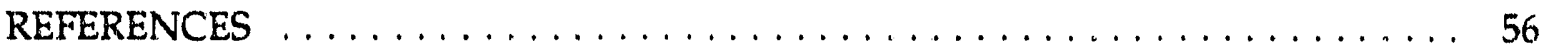

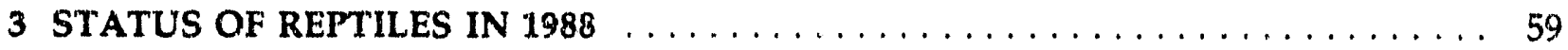

INTRDDUCTION $\ldots \ldots \ldots \ldots \ldots \ldots \ldots \ldots \ldots \ldots \ldots \ldots \ldots \ldots \ldots$

METHODS .................................. 59

Sampling Locations and Dates $\ldots \ldots \ldots \ldots \ldots \ldots \ldots \ldots \ldots \ldots \ldots$

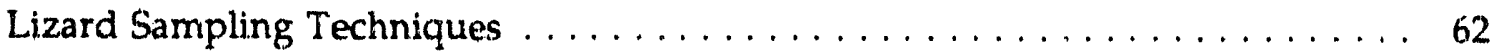

Adult Uta: Baseline Monitoring Plots . . . . . . . . . . . . . . 62

Hatchling Uta: Baseline Monitoring Plots . . . . . . . . . . . . . 66

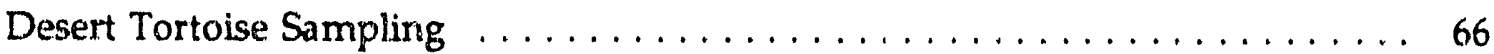

RESULTS AND DISCUSSION $\ldots \ldots \ldots \ldots \ldots \ldots \ldots \ldots \ldots \ldots \ldots$

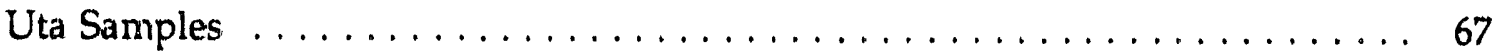

Baseiine Monitoring Plots: Spring $1988 \ldots \ldots \ldots \ldots \ldots \ldots \ldots$. . . . . . . . 67

Baseline Monitoring Plots: Summer $1988 \ldots \ldots \ldots \ldots \ldots \ldots$

Survivorship Of Adult Uta in $1988 \ldots \ldots \ldots \ldots \ldots \ldots \ldots \ldots$

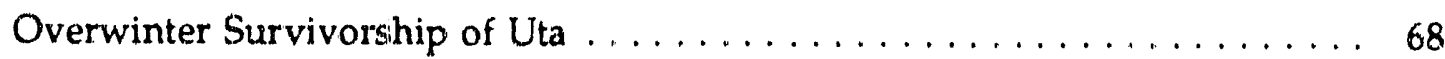

Subsidiary Study Plots in Yucca Flat . . . . . . . . . . . . . . . 69

Blast Area Study at $T 1 . \ldots \ldots \ldots \ldots \ldots \ldots \ldots \ldots \ldots \ldots$

Blast, Bladed and Undisturbed Plots in Area $3 \ldots \ldots \ldots \ldots \ldots$

Lizard Transects (Baselirie Monitoring Plots) . . . . . . . . . . . . . . 73

Feptile Species Observed on the Nevada Test Site $\ldots \ldots \ldots \ldots \ldots \ldots \ldots$. . . . . 74

Desert Tortoise Study . . . . . . . . . . . . . . . . . . . . . . 74

Rock Valley (Fenced Study Areas) $\ldots \ldots \ldots \ldots \ldots \ldots \ldots \ldots \ldots$

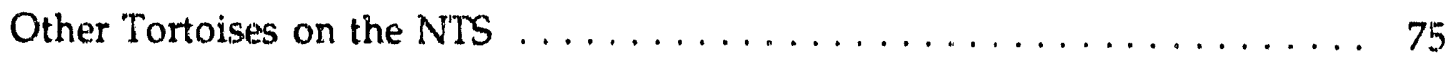

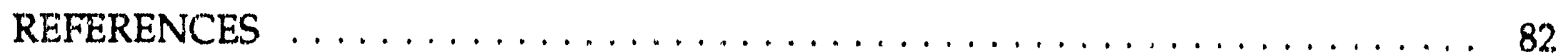




\section{TABLE OF CONTENTS (Continued)}

4 STATUS OF SMALL MAMMALS ON THE NTS IN $1988 \ldots \ldots \ldots \ldots \ldots$

INTRODUCTION $\ldots \ldots \ldots \ldots \ldots \ldots \ldots \ldots \ldots \ldots \ldots \ldots \ldots \ldots$

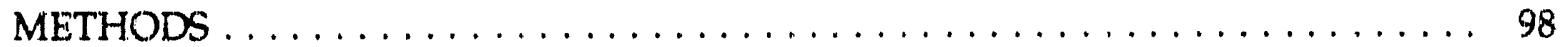

Small Mammal Sampling Techniques $\ldots \ldots \ldots \ldots \ldots \ldots \ldots \ldots \ldots$

Baseline Monitoring Plots . . . . . . . . . . . . . . . . . 98

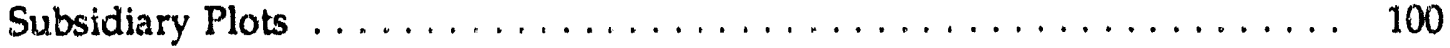

Trap Density Experiment . . . . . . . . . . . . . . . . . . 100

Density Estimation . . . . . . . . . . . . . . . . . . . . . 102

Diumal Rodents (Ground Squirrels) . . . . . . . . . . . . . . . . . . 102

Rabbit and Hare Sampling (Transect Lines) . . . . . . . . . . . . . 103

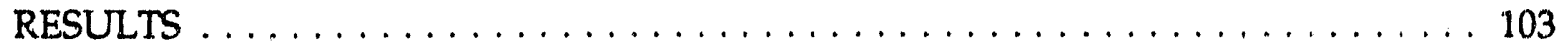

Baseline Monitoring Plots $\ldots \ldots \ldots \ldots \ldots \ldots \ldots \ldots \ldots \ldots \ldots \ldots$

Subsidiary Monitoring Sites - Disturbed and Undisturbed Plots . . . . . . . . 108

Disturbance Due To Fire $\ldots \ldots \ldots \ldots \ldots \ldots \ldots \ldots \ldots \ldots$

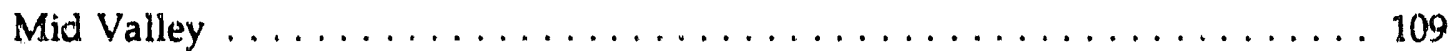

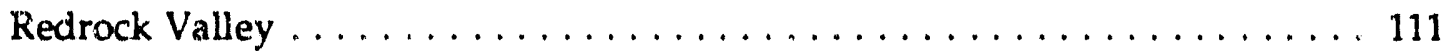

Rock Valley (Fenced, Previously lrradiated Plot and Control) . . . . . . . 115

Disturbance From Aboveground Testing and Blading . . . . . . . . . 118

Continued Monitoring of Disturbance From the Sedan Cratering Test . . . . 123

Disturbances from Naturally Occurring Gopher Populations . . . . . . . . . . 126

Trap Density Experiment . . . . . . . . . . . . . . . . . . . . . . . . . 127

Squirrel Trapping . . . . . . . . . . . . . . . . . . . . . . . . . . . . 129

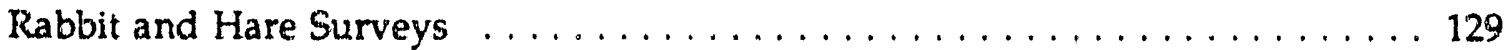

DISCUSSION AND RECOMMENDATIONS $\ldots \ldots \ldots \ldots \ldots \ldots \ldots \ldots \ldots$

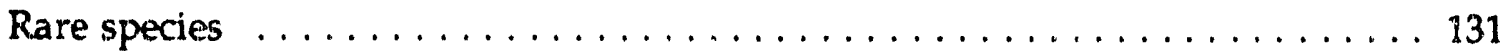

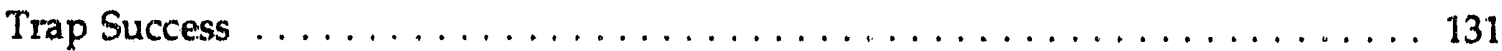

Type of Disturbance Related Differences . . . . . . . . . . . . . . 131

Future Monitoring Plan . . . . . . . . . . . . . . . . . . . . . . . . 133

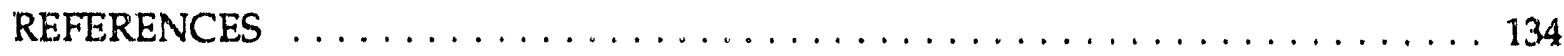

5 WILDLIFE UTILIZATION OF NATURAL SPRINGS AND MAN-MADE WATER SOURCES AT THE NEVADA TEST SITE $\ldots \ldots \ldots \ldots \ldots \ldots \ldots \ldots \ldots \ldots \ldots \ldots$

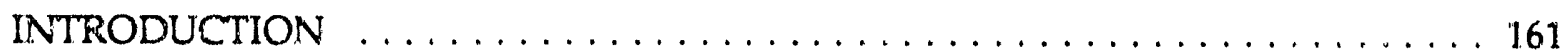

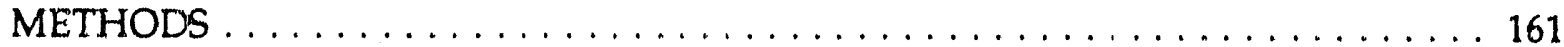

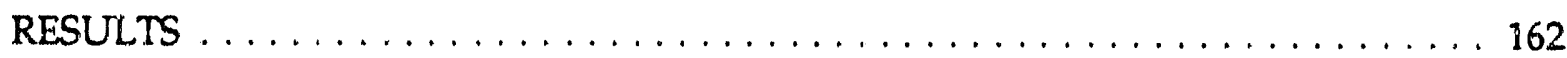

DISCUSSION $\ldots \ldots \ldots \ldots \ldots \ldots \ldots \ldots \ldots \ldots \ldots \ldots \ldots \ldots \ldots \ldots$ 


\section{TABLE OF CONTENTS (Continued)}

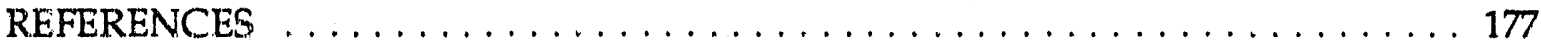

6 STATUS OF PERENNIAL PLANTS ON THE NEVADA TEST SITE IN $1988 \ldots \ldots$

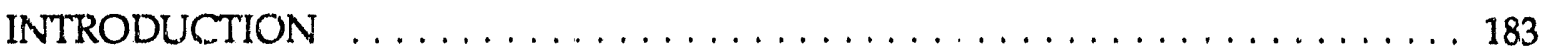

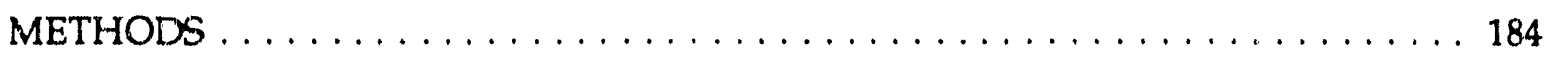

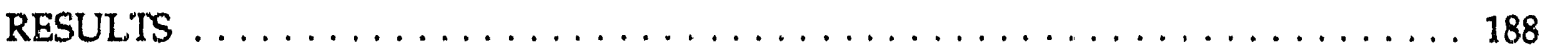

Measurements at Previously Sampled Locations $\ldots \ldots \ldots \ldots \ldots \ldots \ldots \ldots$

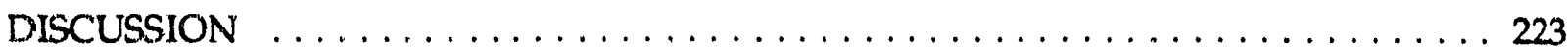

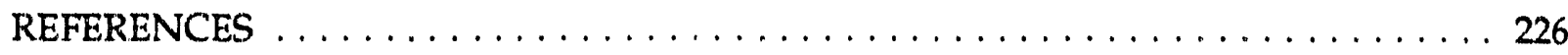

\section{FIGURES}

Figure 1.1 Area Designations on the Nevada Test Site $\ldots \ldots \ldots \ldots \ldots \ldots \ldots$

Figure 2.1 Ephemeral plant study sites for $1988 \ldots \ldots \ldots \ldots \ldots \ldots \ldots \ldots \ldots$

Figure 3.1 BECAMP study plots sampled for lizards in $1988 \ldots \ldots \ldots \ldots \ldots \ldots$

Figure 3.2 BECAMP pristine monitoring plot layout. $\ldots \ldots \ldots \ldots \ldots \ldots \ldots$

Figure 3.3 Dorsal view of lizard toes illustrating the toe numbering formula. . . . . . 63

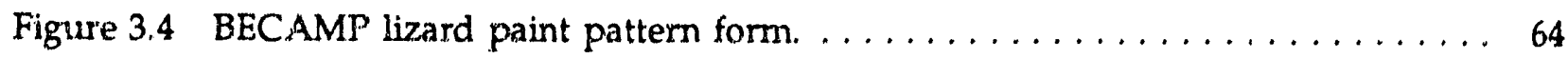

Figure 3.5 Symbols used to paint adult $\underline{\text { ta. }} \ldots \ldots \ldots \ldots \ldots \ldots \ldots \ldots \ldots$

Figure 3.6 Fireball as a result of the nuclear test Apple II (May 5, 1955) which enveloped the

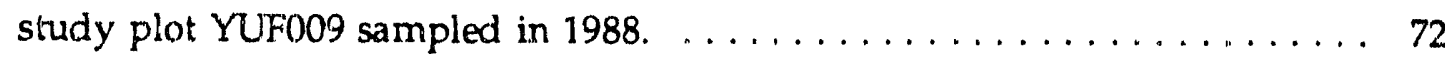

Figure 3.8 Tortoise capture locations on the NTS in $1988 \ldots \ldots \ldots \ldots \ldots \ldots 77$

Figure 4.1 Locations of 1988 BECAMP small mammal study plots. . . . . . . . . . . . . . 99

Figure 4.2 Placement of the $4 \times 4,5 \times 5,7 \times 7$, and $10 \times 10$ grids inside of the larger, $12 \times 12$, grids on the Jackass Flats and Frenchman Flat baseline monitoring plots. . . 101

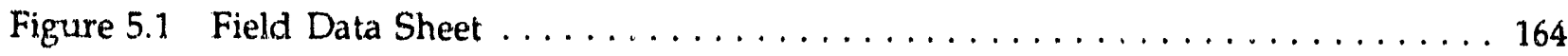

Figure 5.2 Natural springs at the Nevada Test Site $\ldots \ldots \ldots \ldots \ldots \ldots \ldots$

Figure 5.3 Man-made well and pond water sources at the Nevada Test Site . . . . . . 166

Figure 5.4 Sightings of individual raptors during $1988(n=87) \ldots \ldots \ldots \ldots 17$

Figure 5.5 Mule deer utilization of water sources at the Nevada Test Site . . . . . . 172

Figure 5.6 Feral horse utilization of water sources at the Nevada Test Site . . . . . . . 173

Figure 5.7 Feral horse sightings at the Nevada Test Site $(n=33) \ldots \ldots \ldots \ldots \ldots$

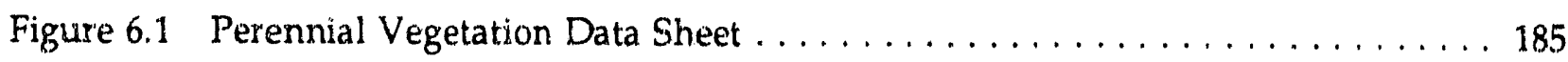

Figure 6.2 BECAMP study plots sampled for perennial plants in $1988 \ldots \ldots \ldots$. . . . . . 187 


\section{TABLE OF CONTENTS (Continued)}

\section{TABLES}

Table 1.1 Estimated extent of various disturbances on the Nevada Test Site $\ldots \ldots \ldots$

Table 2.1 Ephemeral population characteristics on Plot ROV005, Beatley Plot 3 in Rock Valley, measured April $11,1988 \ldots \ldots \ldots \ldots \ldots \ldots \ldots \ldots$

Table 2.2. Ephemeral population characteristics on Plot ROV006, Beatley Plot 4 in Rock

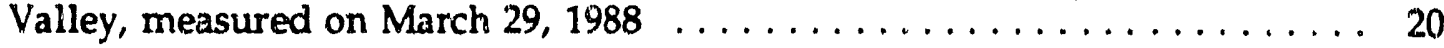

Table 2.3 Ephemeral population characteristics on Plot JAF001, Jackass Flats baseline plot,

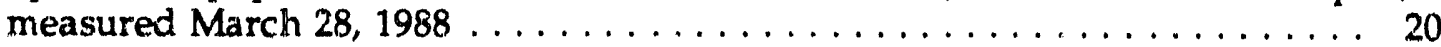

Table 2.4 Ephemeral population characteristics on Plot MER002, Mercury gopher area,

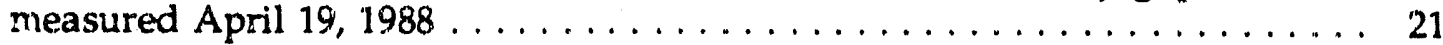

Table 2.5 Ephemeral population characteristics for Plot MER001a, Mercury water balance plot control, measured April $7,1988 . \ldots \ldots \ldots \ldots \ldots \ldots \ldots \ldots \ldots \ldots$

Table 2.6 Ephemeral population characteristics on Plot MER001b, Mercury water balance plot with shrubs removed, measured April 5, $1988 \ldots \ldots \ldots \ldots \ldots \ldots \ldots$

Table 2.7 Ephemeral population characteristics on Plot FRF001a, Frenchman Flat baseline plot (Beatley Plot 23), replicate 1, measured April 13, $1988 \ldots \ldots \ldots \ldots$

Table 2.8 Ephemeral population characteristics on Plot FRF001, replicate 2, measured April 13,1988

Table 2.9 Ephemeral population characteristics on Plot FRF002a, Frenchman Flat roadside,

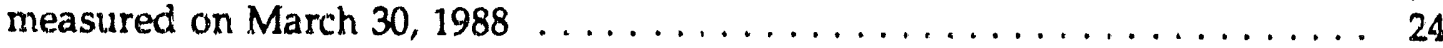

Table 2.10 Ephemeral population characteristics on Plot FRF002b, Frenchman Flat roadside control, measured on March 30, $1988 \ldots \ldots \ldots \ldots \ldots \ldots \ldots \ldots$

lable 2.11 Ephemeral population characteristics from Plot MID001a, Mid Valley burned area, measured on May 5, 1988.

Table 2.12 Ephemeral population characteristics on Plot MID001b, Mid Valley control area, May 5, 1938. (Herbaceous perennials are not included in the totals.) . . . . 27

Table 2.13 Ephemeral population characteristics on Plot YUF001, Yucca Flat baseline plot, replicate 1, measured April $14,1988 \ldots \ldots \ldots \ldots \ldots \ldots \ldots \ldots \ldots$

Table 2.14 Ephemeral population characteristics on Plot YUF001, replicate 2, measured on

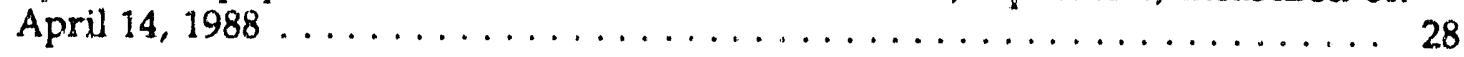




\section{TABLE OF CONTENTS (Continued)}

Table 2.15 Ephemeral population characteristics on Plot YUF009, T1 ground zero, measured

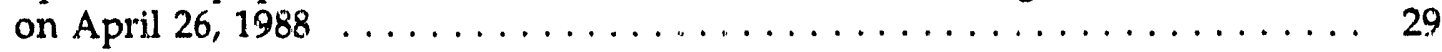

Table 2.16 Ephemeral population characteristics on Plot YUF010, T1 ground zero control,

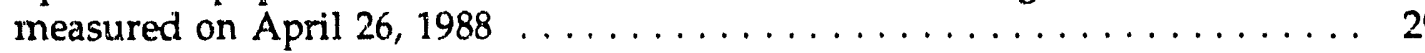

Table 2.17 Ephemeral population characteristics on Plot YUF011, 3B waste consolidation

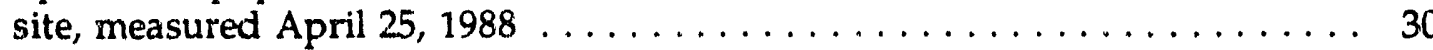

Table 2.18 Ephemeral population characteristics on Plot YUF012, 3B waste consolidation site control area, measured on April $25,1988 . \ldots \ldots \ldots \ldots \ldots \ldots \ldots \ldots \ldots \ldots \ldots$

Table 2.19 Ephemeral population characteristics on Plot YUF014, T2-1 ground zero,

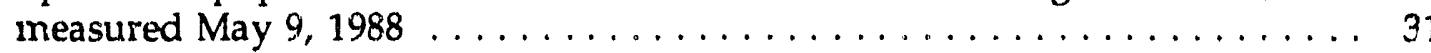

Table 2.20 Ephemeral population characteristics on Plot YUF015, T2-1 control area,

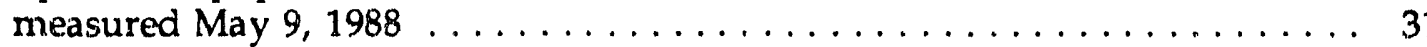

Table 2.21 Ephemeral population characteristics on Plot YUF016, Sedan, 305 meters from ground zero, measured on April 28, $1988 \ldots \ldots \ldots \ldots \ldots \ldots \ldots \ldots \ldots \ldots$

Table 2.22 Ephemeral population characteristics on Plot YUF017, Sedan, 914 meters from ground zero, measured on April $28,1988 \ldots \ldots \ldots \ldots \ldots \ldots \ldots \ldots \ldots$

Table 2.23 Ephemeral population characteristics on Plot YUF018, Sedan, 1524 meters from ground zero, measured May $2,1988 \ldots \ldots \ldots \ldots \ldots \ldots \ldots \ldots \ldots \ldots$

Table 2.24 Ephemeral plant population characteristics on Plot PAM001, Pahute Mesa baseline plot, measured on May 23, 1988

Table 2.25 Ephemeral and herbaceous perennial population characteristics on Plot RAM001, Rainier Mesa baseline plot, measured May 25, $1988 \ldots \ldots \ldots \ldots \ldots \ldots$

Table 2.26 Dead annuals on a site within a burned area in a valley within Yucca Mountain. The fire occurred June 21; dead grass was measured June 29, $1988 \ldots \ldots \ldots 34$

Table 2.27 Species presence-absence on plots sampled in 1988 for ephemeral plants. ( $\mathrm{A}=$ censused in quadrats (densities and biomasses are in Tables 2.1-2.26); $\mathrm{B}=$ present in $100 \mathrm{~m}^{2} ; \mathrm{C}=$ present in $1000 \mathrm{~m}^{2} ; \mathrm{D}=$ present only near the plot; - = not seen; * $=$ introduced species of ephemerals encountered in 1988$) \ldots \ldots \ldots \ldots$

Table 2.28 Species presence-absence on plots sampled in 1988 for ephemeral plants. $(\mathrm{A}=$ censused in quadrats (densities and biomasses are in Tables 2.1-2.26); $\mathrm{B}=$ present in $100 \mathrm{~m}^{2} ; C=$ present in $1000 \mathrm{~m}^{2} ; \mathrm{D}=$ present only near the plot; $-=$ not

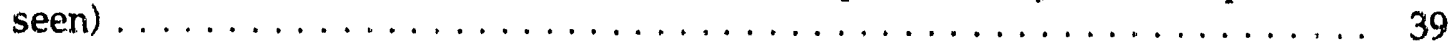




\section{TABLE OF CONTENTS (Continued)}

Table 2.29 Species presence-absence on plots sampled in 1988 for ephemeral plants. $(\mathrm{A}=$ censused in quadrats (densities and biomasses are in Tables 2.1-2.26); $\mathrm{B}=$ present in $100 \mathrm{~m}^{2} ; \mathrm{C}=$ present in $1000 \mathrm{~m}^{2} ; \mathrm{D}=$ present only near the plot; - = not

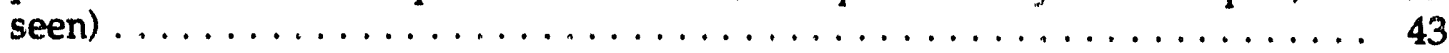

Table 2.30 Species presence-absence on plots sampled in 1988 for ephemeral plants. $(A=$ censused in quadrats (densities and biomasses are in Tables 2.1-2.26); $B=$ present in $100 \mathrm{~m}^{2} ; \mathrm{C}=$ present in $1000 \mathrm{~m}^{2} ; \mathrm{D}=$ present only near the plot; - = not

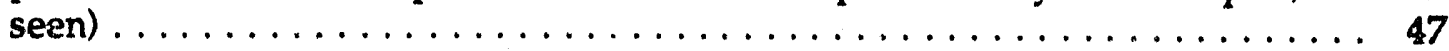

Table 2.31 Estimate 1 soil water (0-30 cm depth; total $\mathrm{mm}$ of water 1-15) and precipitation (mm) in Mercury, Nevada between July 1, 1987, and June 30, 1988

Table 2.32 Densities (number $/ \mathrm{m}^{2}$ ) of Bromus rubens on Beatley Plots 3 and 4 in Rock Valley (ROV005 and ROV006), 1963-1988. Errors are sem . . . . . . . . . . 52

Table 2.33 Average weights ( $\mathrm{mg}$ ) of Bromus plants when growing at the same location . 53

Table 2.34 Numbers of ephemeral species and percent introduced biomass on sites where ephemerals were sampled in 1988

Table 2.35 Densities of Bromus rubens and native species censused in several close plots in Rock Valley, 1963-1988 ....................... 55

Table 3.1 Dates BECAMP study plots were sampled for Uta stansburiana in $1988 \ldots \ldots 2$

Table 3.2 Estimated densities (number of lizards per hectare \pm two standard errors) of Uta stansburiana in the spring (March) of 1988 on three baseline monitoring plots (1.10 ha) on the Nevada Test Site. The number in parentheses is the total number of individuals enumerated $\ldots \ldots \ldots \ldots \ldots \ldots \ldots \ldots \ldots \ldots$

Table 3.3 Estimated densities (number of lizards per hectare \pm two standard errors) of Uta stansburiana in summer (August-September) 1988 on four baseline monitoring plots ( $1.10 \mathrm{ha})$ on the Nevada Test Site. Numbers in parentheses are the total numbers of individuals enumerated

Table 3.4 Number and percent survivorship of marked adult Uta stansburiana from spring (March 1988) to late summer (August - September 1988) on three baseline monitoring plots $(1.10 \mathrm{ha})$ on the Nevada Test Site $\ldots \ldots \ldots \ldots 6$

Table 3.5 Number and overwinter survivorship (\%) of adult and juvenile Uta stansburiana on three baseline monitoring plots between August 1987 and March 1988 . . 69

Table 3.6 Survivorship of adult Uta stansburiana between March and August 1988 on the baseline monitoring plots compared to ephemeral biomass and numbers/meter 


\section{TABLE OF CONTENTS (Continued)}

Table 3.7 Estimated densities (number per hectare \pm two standard errors) of Utu stansburiana on subsidiary ( $0.56 \mathrm{ha}$ ) study plots YUF002 (burned) and YUF003 (unburned) in spring and summer of 1988 . Numbers in parentheses are actual numbers of individuals enumerated $\ldots \ldots \ldots \ldots \ldots \ldots \ldots \ldots \ldots$

Table 3.8 Estimated densities (number per hectare \pm two standard errors) of Uta stansburiana in late April and August-September on subsidiary ( 0.56 ha) plots YUF009 (T1 Blast Area) and YUF010 (Control). Numbers in parentheses are actual numbers of individuals enumerated ................. 72

Table 3.9 Estimated densities (number per hectare \pm two standard errors) of Uta stansburiana in spring (early May) on subsidiary (0.56 ha) plots YUF011 (38 consolidation site), YUF012 (Undisturbed), and YUF013 (T3a Blast Area). Numbers in parentheses are actual numbers of individuals enumerated . . . 73

Table 3.10 Relative abundance of adult lizards observed on the BECAMP baseline monitoring plot transect lines surveyed in 1988. Estimated numbers/ha in parentheses

Table 3.11 Lizards that were observed on the BECAMP baseline monitoring plots in 1988 are indicated by $(X)$; those which probably were present but were not observed (P). Blanks indicate that the species probably does not occur on the plo! . . 78

Table 3.12 Desert tortoise and snakes that were observed on the BECAMP baseline monitoring plots in $1988(\mathrm{X})$; those which probably were present but were not observed (P), and species which probably did not occur (blank) . . . . . 79

Table 3.13 Desert tortoises (Gopherus agassizii) captured on the Nevada Test Site in 1988

Table 4.1 Abbreviation for scientific names of small mammals captured on NTS and the common name

Table 4.2 Estimated densities (number per hectare \pm two standard errors) of small mammals on BECAMP baseline monitoring plots on NTS in 1988 . Numbers in parentheses are individual animals captured. . . . . . . . . . 105

Table 4.3 Percent of total captured population (\% $\mathrm{T}$ ) and sex ratio (M/F = male:female) of small mammals on the BECAMP baseline monitoring plots on the NTS in 1988

Table 4.4 Adult spring mean weights (grams $\pm 2 \mathrm{SE}$ ) by sex of common rodent species on three BECAMP baseline monitoring plots in $1988 \ldots \ldots \ldots \ldots \ldots$

Table 4.5 Estimated densities (number/hectare $\pm 2 \mathrm{SE}$ ) of small mammals on the BECAMP plots (1.67 ha) at the Mid Valley burn site, MIDO02 and MIDO03, during June 1988. Numbers in parentheses are individual animals captured . . . . . . . 109 


\section{TABLE OF CONTENTS (Continued)}

Table 4.6 Percent of total captured population $(\% \mathrm{~T})$ and sex ratio $(\mathrm{M} / \mathrm{F}=$ male : female) of small mammals on the BECAMP plots in Mid Valley in $1988 \ldots \ldots \ldots \ldots 111$

Table 4.7 Estimated densities (number per hectare $\pm 2 \mathrm{SE}$ ) of small mammals on the BECAMP plots (1.58 ha) at the Redrock Valley burn site during trapping in July, August, and October 1988. Numbers in parentheses are individual animals

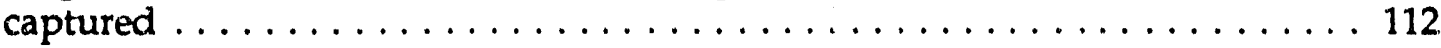

Table 4.8 Percent of total captured population (\% $\mathrm{T})$ and sex ratios $(\mathrm{M} / \mathrm{F}=$ male : female) of small mammals on the BECAMP plots in Redrock Valley on NTS in 1988 . . 114

Table 4.9 Estimated densities (number/ha $\pm 2 \mathrm{SE}$ ) of small mammals inside the 3.24-ha Rock Valley fenced plot (3.24 ha) in June 1988 and on IBP plot 16 in July 1972, April and August 1973, and June 1988. Numbers in parentheses are individual

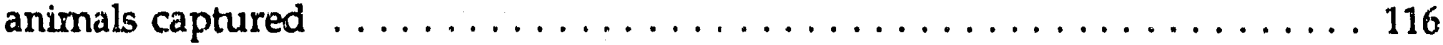

Table 4.10 Percent of total captured population (\%T) and sex ration $(\mathrm{M} / \mathrm{F}=$ male : female) of small mammals inside the Rock Valley fenced plot (ROVO08) in 1988 and on IBP plot 16 (ROV007) in 1972, 1973, and $1988 \ldots \ldots \ldots \ldots \ldots \ldots \ldots \ldots$

Table 4.11 Estimated densities (number/ha \pm 2 SE) of small mammals on the BECAMP subsidiary plots (1.44 ha) at the tower shot area, T1, in Yucca Flat in May 1988. Numbers in parentheses are individual animals captured . . . . . . . . . . 119

Table 4.12 Percent of total captured population $(\% \mathrm{~T})$ and sex ratio $(\mathrm{M} / \mathrm{F}=$ male : female) of small mammals on the BECAMP plots at T1, Yucca Flat in $1988 \ldots \ldots \ldots \ldots$

Table 4.13 Estimated densities (number/hectare \pm 2 SE) of small mammals on the BECAMP plots (1.58 ha) in Yucca Flat, YUF011, YUF012, and YUF013, in May 1988.

Numbers in parentheses are the number of individual animals captured . . . 121

Table 4.14 Percent of total captured population $(\% ' \Gamma)$ and sex ratio $(M / F=$ male : female) on BEC.AMP plots YUF011, YUF012, and YUF013 on Yucca Flat in $1988 \ldots \ldots \ldots 122$

Table 4.15 Estimated densities (number/hectare \pm 2 SE) of small mammals on the BECAMP plots (1.44 ha) at Sedan crater, YUF016, XUF017, and YUF018 during July, 1988. Numbers in parentheses are number of individuals captured $\ldots \ldots \ldots \ldots 123$

Table 4.16 Percent of total captured population (\% $\mathrm{T})$ and sex ratio $(\mathrm{M} / \mathrm{F}=$ male : female) of small mammals on the BECAMP plots at Sedan crater in Yucca Flat in 1988 . 124

Table 4.17 Population estimate of small mammals (number per hectare) along line 16A before and after detonation of Sedan. Densities were calculated from number per

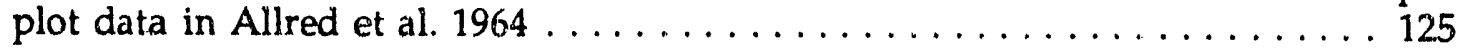




\section{TABLE OF CONTENTS (Continued)}

Table 4.18 Estimated densities (number/ha \pm 2 SE) of small mammals on the BECAMP plots (1.44 ha) at a gopher site in Mercury Valley in August 1988. Numbers in

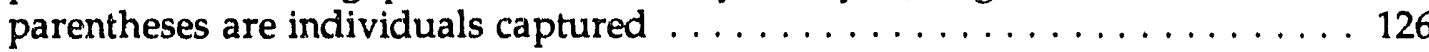

Table 4.19 Percent of total captured population (\%T) and sex ratios (M/F = male : female) of small mammals on the BECAMP plots at a gopher disturbed site in Mercury Valley on NTS in 1988

Table 4.20 Number of animals per hectare and percent trap success during one day of trapping on $4 \times 4,5 \times 5,7 \times 7$, and $10 \times 10$ grids and three days of trapping on a $12 \times 12$ grid. Densities were calculated using actual grid size without any

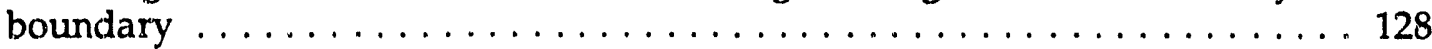

Table 4.21 Estimated densities ( $D=$ number/hectare $\pm 1 S E)$ and mean flushing distance $(F=$ meters $\pm 1 \mathrm{SE})$ of rabbits and hares on BECAMP baseline sites on NTS in June 1988

Table 4.22 Number of species, average capture rates, and percent trap success on baseline, disturbed, and control monitoring plots in 1988 on NTS . . . . . . . . . 132

Table 5.1 Approximate elevation and location of natural springs on the Nevada Test Site monitored for wildlife utilization . . . . . . . . . . . . . . . . . 167

Table 5.2 Approximate elevation and location of well reservoirs on the Nevada Test Site monitored for wildlife utilization $\ldots \ldots \ldots \ldots \ldots \ldots \ldots \ldots \ldots$

Table 5.3 Total numbers of wildlife observed at natural springs at the Nevada Test Site during 1988. $P$ indicates utilization by a species inferred from animal sign . . . . . . . . . . . . . . . . . . . . . . . . . 169

Table 5.4 Total numbers of wildlife observed at well reservoirs on the Nevada Test Site during 1988. $P$ indicates utilization by a species inferred from

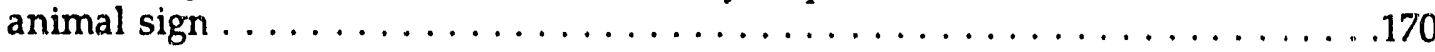

Table 6.1 Dominant species of perennial plants found on the Nevada Test Site. . . . 184

Table 6.2 Perennial plant species names, authorities, and the abbreviations used in

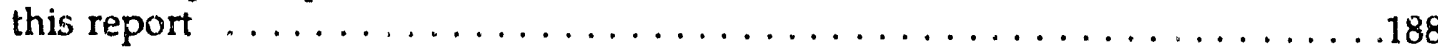

Table 6.3 Analysis of unmatched plants from 1987 and 1988 censuses on transect JAF001 V1. Missed plants in 1988 were present in 1987, and new 1988 plants were presumably missed in $1987 \ldots \ldots$. . . . . . . . . . . . . . . . . . . 190

Table 6.4 Perennial plant population characteristics on a $100 \mathrm{~m}^{2}$ transect (JAF001 V1) at an elevation of about $954 \mathrm{~m}$ sampled July 31, 1987 (top line), and July 13, 1988 (second line). Totals exclude dead plants 


\section{TABLE OF CONTENTS (Continued)}

Table 6.5 Perenrial plant population characterisícs on a $100 \mathrm{~m}^{2}$ transiect (JAFO0)1 V4), a baseline plot at an elevation of $954 \mathrm{~m}$, sampled July 23, 1987 (first line), and July 11, 1988 (second line). Totals exclude dead plants.

Table 6.6 Perennial plant population characteristics on a 100-m ${ }^{2}$ transect (FRF001 V1), at an elevation of $965 \mathrm{~m}$, sampled June 10, 1987 (top line) and June 23, 1988 (second line). Totals exclude dead plants ....................... 194

Table 6.7 Perennial plant population characteristics on a $100-\mathrm{m}^{2}$ transect (YUFO01 V2), at an elevation of $1237 \mathrm{~m}$ on a Yucca Flat baseline site. It was sampled on June 6, 1987 (first line) and July 19, 1988 (second line), Totals exclude dead plants . . . . $190^{\circ}$

Table 6.8 Perennial plant population characteristics on a $100-\mathrm{m}^{2}$ transect (XUFOO1 V3), at 1237 m elevation, sampled on August 7, 1987 (top line) and July 22, 1988 (second line). Totals exclude dead plants. . . . . . . . . . . . . . . . . 198

Table 6.9 Perennial plant population characteristics on a 200- $\mathrm{m}^{2}$ transect, YUF012, at an elevation of $1239 \mathrm{~m}$, sampled May 14, 1986 (top line) and August 8, 1988 (second line). Totals are not adjusted to $100 \mathrm{~m}^{2}$ area, and exclude dead plants. . . . 200

Table 6.10 Growth coefficients $\left(k=\left[\ln \left(V_{2} / V_{1}\right)\right] /\right.$ time in years; mean \pm sem) of species inhabiting the six resampled transects, based on matched plants at two cens'ises. Plot YUF012 was sampled 1986 and 1988, and all others 1987 and $1988 \ldots \ldots 203$

Table 6.11 Times to double in size associated with different growth coefficients $(k=$ $\| \ln (\mathrm{V} 2 / \mathrm{V} 1)] /$ time in years). . . . . . . . . . . . . . . . . . . . 204

Table 6.12 Perennial plant population characteristics on transect FRF002 (Roadside), $100 \mathrm{~m}^{2}$ measured on the pavement edge of an abandoned road at an elevation of $977 \mathrm{~m}$,

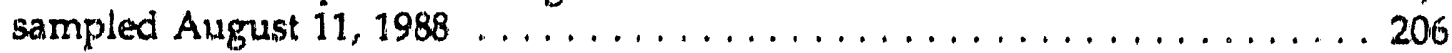

Table 6.13 Perennial plant population characteristics on a $100-\mathrm{m}^{2}$ transect on plot FRFOO2 (roadside control), at an elevation of $977 \mathrm{~m}$, sampled August 12, 1988. 'Totals exclude dead plants. . . . . . . . . . . . . . . . . . . . . . . . . . . . . . 207

Table 6.14 Perennial plant population characteristics on a $100 \mathrm{~m}^{2}$ transect in a rodentdenuded area (MEROO2) at an elevation of $1076 \mathrm{~m}$, sampled july 20, 1988. Totals exclude dead shrubs. . . . . . . . . . . . . . . . . . . . . . . . . . 208

Table 6.15 Perennial plant population characteristics on a $100-\mathrm{m}^{2}$ transect (MERO03), a cortrol for MEROO2, at an elevation of $1103 \mathrm{~m}$ sampled July 28, 1988. Totals exclude dead plants . . . . . . . . . . . . . . . . . . . . . 209

Table 6.16 Perennial plant population characteristics on a $100 \mathrm{~m} \mathrm{~m}^{2}$ transect on plot YUFO13, Area 3 ground zero, at an elevation of $1236 \mathrm{~m}$, sampled August $8,1988 . \ldots 210$ 


\section{TABLE OF CONTENTS (Continued)}

Table 6.17 Perennial plant population characteristics on a transect at T1 blast area (YUF009), at an elevation of about $1279 \mathrm{~m}$, sampled August 12, 1988. This transect covered $200 \mathrm{~m}^{2}$. Totals exclude dead plants, and were not adjusted to $100-\mathrm{m}^{2}$ area . 211

Table 6.18 Perennial plant population characteristics on a $100-\mathrm{m}^{2}$ transect in undisturbed vegetation east of $\mathrm{T} 1$ ground zero. The plot (YUFO10) is at an elevation of about $1267 \mathrm{~m}$, and was sampled August 9, 1988. Totals exclude dead plants . . . 212

Table 6.19 Grass seedlings marked following three germination events and censused the two succeeding years on $\mathrm{T} 1$ ground zero . . . . . . . . . . . . . 213

Table 6.20 Perennial plant numbers and percent cover on five $100-\mathrm{m}^{2}$ plots on the T1 blast area, west of ground zero, sampled in 1986 (A-C) and 1987 (D, E) by E. M. Romney and R. B. Hunter . . . . . . . . . . . . . . . . . . . . 214

Table 6.21 Perennial plant population characteristics on a $100-\mathrm{m}^{2}$ transect (YUF016) 305 meters northeast of Sedan ground zero, at an elevation of about $1318 \mathrm{~m}$, sampled

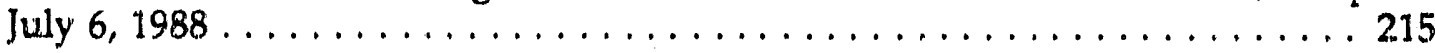

Table 6.22 Perennial plant characteristics on a 100- $\mathrm{m}^{2}$ transect (YUF017) 914 meters northeast of Sedan ground zero at an elevation of about $1327 \mathrm{~m}$, sampled

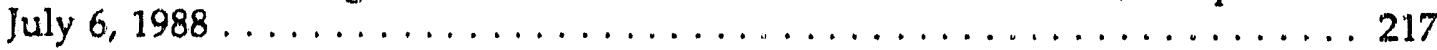

Table 6.23 Perennial plant population characteristics on a 100- $\mathrm{m}^{2}$ transect (YUF018) 1524 meters northeast of Sedan ground zero at an elevation of about $1335 \mathrm{~m}$, sampled July 6,1988 . Totals exclude dead plants. . . . . . . . . . . . . 218

Table 6.24 Perennial plant population characteristics on a $100-\mathrm{m}^{2}$ transect on plot PAM001, a Pahute Mesa baseline area at an elevation of $1923 \mathrm{~m}$, sampled August 16, 1988.

Totals exclude dead plants. . . . . . . . . . . . . . . . . . . . . . . . 219

Table 6.25 Perennial plant population characteristics on a Rainier Mesa baseline plot (RAM001), at an elevation of $2283 \mathrm{~m}$, sampled August 19, 1988. This transect covered only $54 \mathrm{~m}^{2}$. The Penstemon were not flowering and were therefore not identified. Totals are adjusted to $100 \mathrm{~m}^{2}$ area . . . . . . . . . . . . . 220

Table 6.26 Numbers of live plants, live cover (\%), total live volume $\left(\mathrm{m}^{3} / 100 \mathrm{~m}^{2}\right)$, and estimated biomass $\left(\mathrm{kg} / 100 \mathrm{~m}^{2}\right)$ and altitudes (meters) for sites sampled in 1988 


\section{TABLE OF CONTENT'S (Continued)}

\section{APPENDICES}

Appendix 3A BECAMP/Ecology Plot Locations (as of January 1989) . . . . . . . 83

Appendix 3B Density Estimates for Adult Uta stansburiana Sampled in Spring and Summer, and Hatchlings Sampled During the Summer of 1988 on the BECAMP Study Plots on the Nevada Test Site . . . . . . . . . 86

Appendix 4A Species and Number Captured During Three Days of Spring Sampling and One Day of Summer Sampling on Five Baseline Monitoring Plots on NTS in

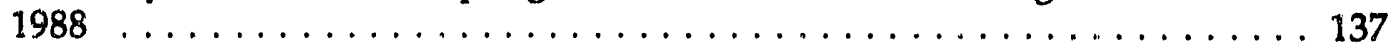

Appendix 4B Mean Weights of the Most Commonly Captured Species Found on BECAMP Monitoring Plots on NTS in $1988 \ldots \ldots \ldots \ldots \ldots \ldots \ldots$

Appendix 4C Results of Density and Standard Error Calculations Using Seber (1982:138) 142

Appendix 5 NTS Natural Springs Samples 1988 (Arranged by site and date, $\mathrm{mg} / \mathrm{liter}$ ) 178 


\section{ACKNOWLEDGEMENTS}

This project was initiated and supported by the Department of Energy, Nevada Field Office, under contract DEAC0889NV10630. The work required the help of many people, including D. D. Badger, L. L. Badger, J. Z. T. Basinger, K. D. Dyka, P. M. Hopkin, D. J. Lopez, J. D. Pinjuv, D. E. Swanson, and T. R. Webb (data collection); F. H. Au, R. O. Gilbert, R. R. Kinnison, R. D. McArthur, and T. P. O'Farrell (statistics and editing); and L. S. Richardson and R. Woolard (manuscript preparation). We are grateful for the help of all those involved. 


\section{ACRONYMS}

BECAMP - Basic Environmental Compliance and Monitoring Program

DOE - Department of Energy

FRF - Frenchman Flat

ha - hectare

MER - Mercury Valley

MID - Mid Valley

NTS - Nevada Test Site

PAM - Pahute Mesa

RAM - Rainier Mesa

RED - Redrock Valley

ROV - Rock Valley

YUF - Yucca Flat 


\section{EXECUTIVE SUMMARY}

This is a report of work performed by Reynolds Electrical \& Engineering Company (REECo) in support of the Department of Energy's (DOE's) Basic Environmental Compliance and Monitoring Program (BECAMIP) Task 3, to monitor the flora and fauna on the Nevada Test Site (NTS). It reports on the second year of efforts to monitor the status of plants and animals on the NTS.

Monitoring efforts were focused in two ways. The primary efforts were to sample ephemeral and perennial plants, resident small mammals, and common lizards on plots scattered throughout the NTS in both baseline and disturbed areas. In addition, wildlife usage was monitored at springs and other water sources on the NTS.

Plots sampled during 1988 included five baseline plots; two areas with vegetation removed by 1950 s' $^{\prime}$ atmospheric nuclear weapons tests; the area surrounding the 1962 Sedan nuclear cratering experiment; a gopher-denuded area; and an area scraped for radioactive waste cleanup. Plants were also sampled on an abandoned roadside in Frenchman Flat, and small mammals were censused on a site in Red Rock Valley where a range fire occurred in July 1988.

Perennial plants on the NTS were generally static from 1987 to 1988 . Shrubs neither grew nor shrank. The only changes were a decrease in numbers of herbaceous perennials such as bunchgrasses and desert globemallow (Sphaeralcea ambigua). These plants are short-lived in comparison to the dominant desert shrubs, and shrink and grow annually in response to weather patterns.

Ephemeral plants produced 0 to 36 grams per square meter on baseline sites, and 0.1 to 92 $\mathrm{g} / \mathrm{m}^{2}$ on disturbed sites. These plants consisted largely of weedy species introduced by man, particularly the grasses Bromus rubens and $B$. tectorum. The densest stands occurred on an area burned in 1986 in a lightning-initiated fire $\left(92 \mathrm{~g} / \mathrm{m}^{2}\right.$ of which $89 \mathrm{~g} / \mathrm{m}^{2}$ was B. tectorum) and on the gopher-denuded area $\left(72 \mathrm{~g} / \mathrm{m}^{2}\right.$, of which $70 \mathrm{~g} / \mathrm{m}^{2}$ was $B$. rubens). These grass densities, if ignited, would probably carry range fires, as an area near Yucca Mountain which did burn in June 1988 was found to have $47 \mathrm{~g} / \mathrm{mm}^{2}$ of dry grass in a protected enclave. A total of 114 species were recorded on 23 plots. The Bromus species occurred in densities of hundreds to thousands per square meter, which was a dramatic change from populations in the 1960 s and 1970 s.

Populations of the common sideblotch lizard (Uta stansburiana) on undisturbed areas ranged from 33 to 122 per hectare in the spring of 1988. Survivorship of adult lizards to August was 8 to 51 percent on control plots. On disturbed areas densities were 0 to 54 per hectare, and survivorship to late summer was lower, 0 and 14 percent on the two areas sampled both seasons. Evidence was that the reduced survivorship was due to increased predation where 
plants were absent. Lizard reproduction was good in 1988. Observations were also recorded on 14 other lizard species and the desert tortoise, which is found on the southern parts of the NTS.

Small mammals trapped on areas cleared of vegetation by nuclear blasts, fire, gophers or scraping all showed a tendency to reduced numbers of two common species, the Great Basin kangaroo rat (Dipodomys microps) and the little pocket mouse (Perognathus longimembris).

Merriam's kangaroo rat (D. merriami), in contrast, was as common on disturbed areas as on control plots. Sampling in the 1960s and 1970s (Sedan and Rock Valley) showed changes have occurred in dominance among the common species, but no consistent trends were apparent. Among rarer species, range extensions were found for weasels (Mustela frenata) from Yucca Flat to Rock Valley and the dark kangaroo mouse (Microdipodops megacephalous sabulonis) from north of the NTS onto Pahute Mesa.

Observations of utilization by wildlife of the natural and man-made water sources on the NTS documented the presence of many species not otherwise studied. The more common species seen were feral horses, chukar, gambels quail, mourning doves, mule deer, and unidentified ducks. Evidences for use of springs by the more secretive species such as mountain lion, bobcat, coyote, and kit fox were also recorded, as were sittings of raptors and migratory waterfowl.

Because 1988 was only the second year of the BECAMP monitoring program, trends in the condition of the NTS flora and fauna could not be identified. 


\title{
SECTION 1 \\ EXTENT OF LAND DISTURBANCE ON THE NEVADA TEST STTE
}

\author{
by \\ Richard B. Hunter and Philip A. Medica
}

The Nevada Test Site comprises approximately 3600 square kilometers divided into 27 geographical areas (Figure 1.1) that range in size from $13 \mathrm{~km}^{2}$ (Area 23, Mercury) to $649 \mathrm{~km}^{2}$ (Area 25, Nevada Research and Development Area, NRDA).

The types of disturbance considered here include burned areas resulting from lightninginitiated fires or man-caused fires; blast zones from nuclear tests radex areas (radiation exclusion areas contaminated by radiation from above-ground nuclear testing); alpha radex areas; waste disposal areas; subsidence craters, drill pads and cable runs; base camp facilities and staging areas; roads (paved and unpaved), pre-emplacement test holes; and drill pads and tests which have not cratered. The area estimates (Table 1.1) are approximations based upon maps and best-guess estimates by personnel who are familiar with the NTS. Fires were approximated by NTS Fire Chief Ray Gudeman, at the time of each fire. Though crude in accuracy, these estimates represent the best available figures as of January 1988. Table 1.1 has associated notes that list the sources (maps, guesses, and measurements made from maps) for the area estimations.

Burned areas comprise the largest single disturbance on the NTS, covering $4.3 \%$ of the total NTS geographical area. The available records only included data from 1978 through 1987. The majority of the fires were in Areas 14, 16, 25, 29 and 30 and in the Mid Valley, Shoshone Mountain and Buckboard Mesa region, which burned nearly 15,600 ha blast zones, at 1 . Altogether, radex areas amount to $0.8 \%$ of the entire area of the NTS; craters and associated activities, $0.6 \%$; and roads, $0.4 \%$. Most radex areas, and many craters and drill pads, are within areas whose vegetation was removed by atmospheric nuclear weapons tests in the 1950s (blast zones).

Virtually all of the NTS has been impacted by introduced plants (although this is difficult to document in hectares). These introduced plants include annual grasses (Bromus rubens, Bromus tectorum, and Schismus arabicus), and weedy ephemerals (Erodium cicutarium, Salsola australis, and Sisymbrium altissimum). The introduced species may considerably change the environment, as they often occur in dense stands. Small portions of the NTS may be affected by introduced mammals (horses) and birds (chukars). Horses may significantly impact areas around springs and other water sources. 
Gophers create large bare patches in the Mojave Desert sections of the NTS. A crude estimate of the total area of these is $400 \mathrm{ha}$, which would be largely in Areas 5, 22 and 25.

The area most heavily impacted by weapons testing is the Yucca Flat valley floor, composed of approximately 57,000 ha. Of that area, about 5724 ha (10\%) have been blasted free of vegetation, 2039 ha $(3.6 \%)$ are affected by subsidence craters and drill pads, and 1403 ha $(2.5 \%)$ are contaminated by radionuclides. Since many of these disturbances overlap, the total percent of the valley floor disturbed by testing must be somewhat less than $15 \%$. 

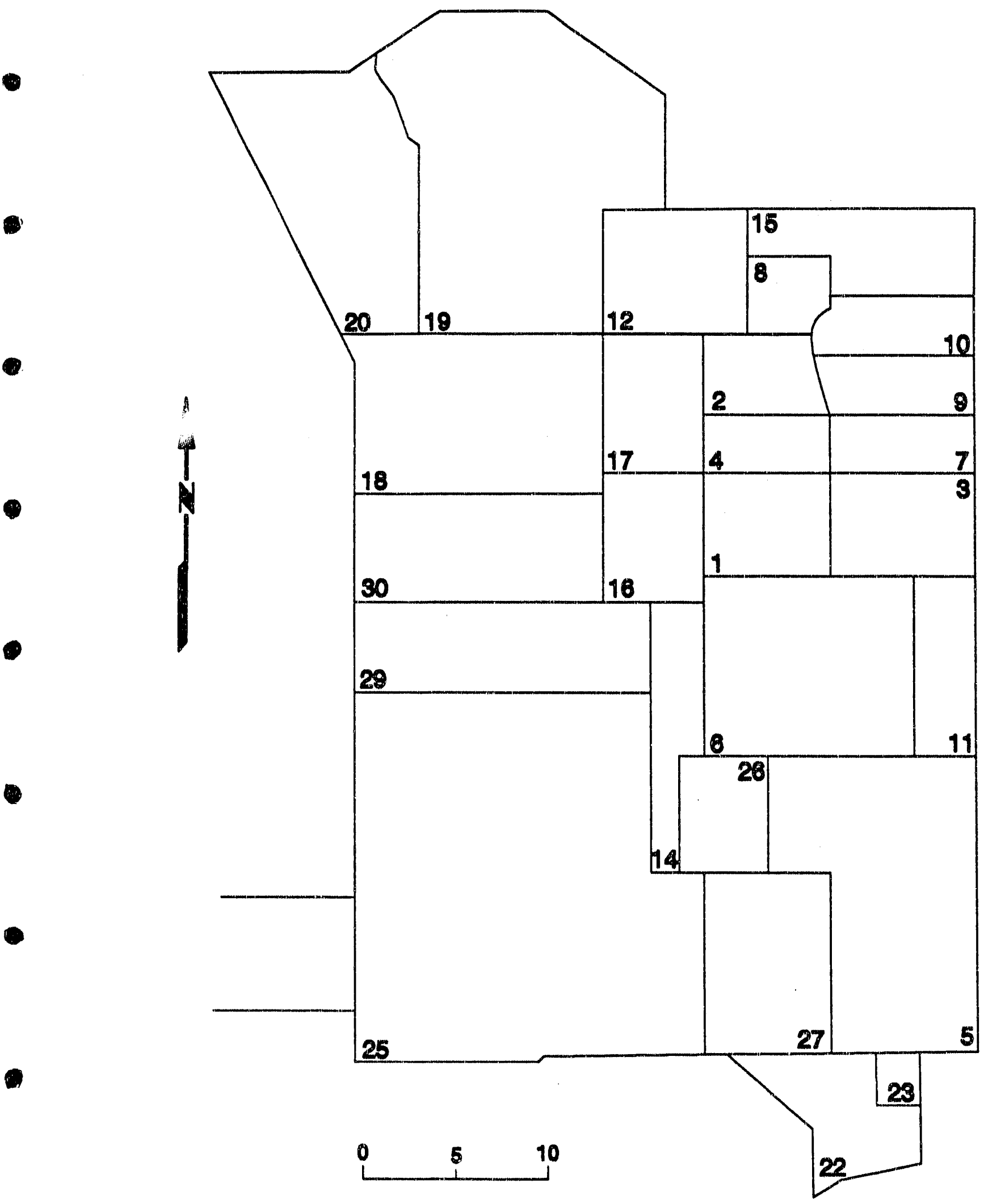

Figure 1.1 Area Designations on the Nevada Test Site 


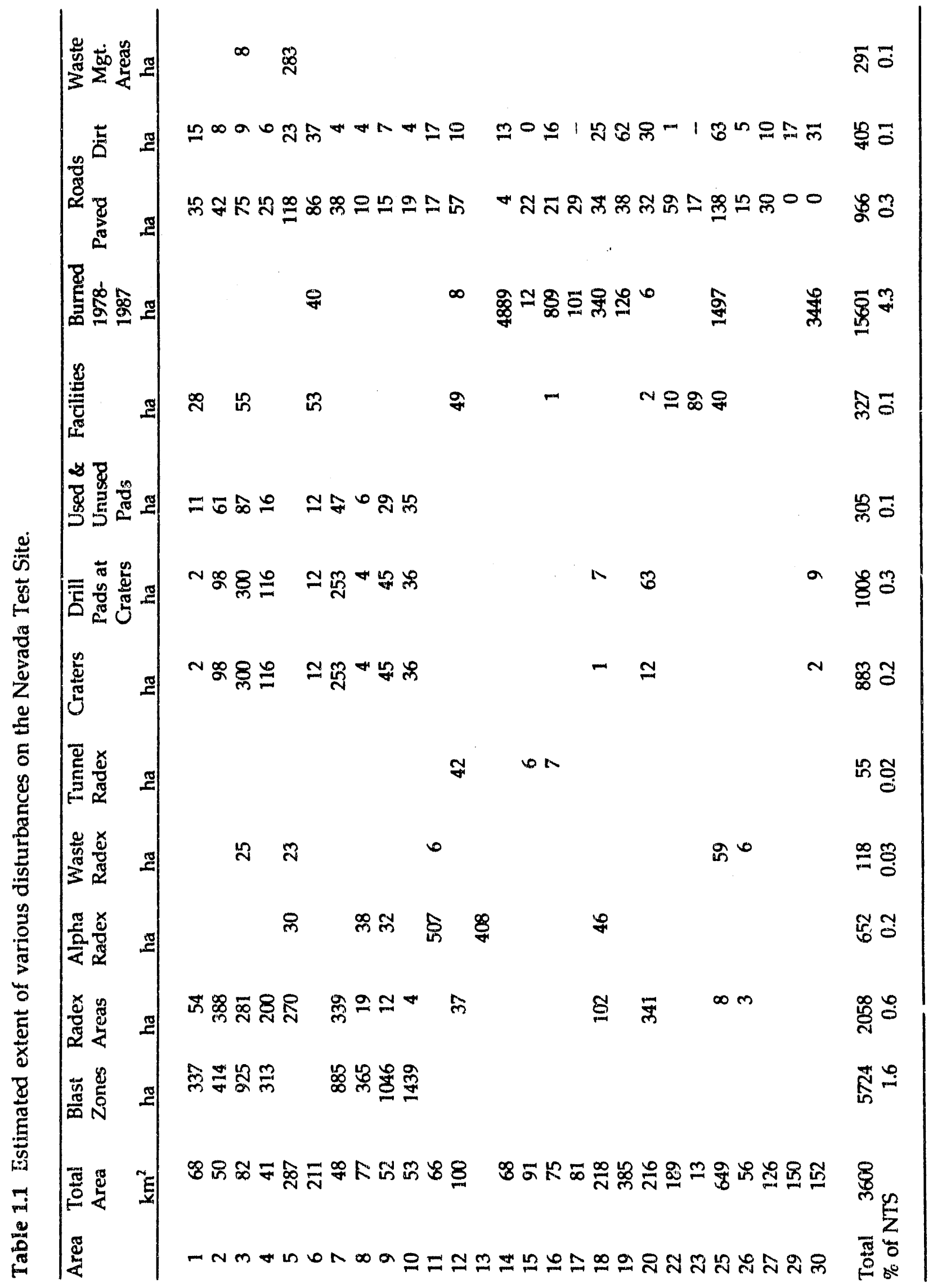


Table 1.1 (notes)

Area:

Area designation on NTS maps.

Total area:

Based upon independent measurements on the NTS Map.

Blast zones:

Based upon map in BYU report, Allred et al. 1963"

Radex areas:

Based upon Radsafe Map, June 1984.

Alpha radex areas:

Based upon Radsafe Map, June 1984. (Area 11 area estimate is based upon the area beyond Barricade 11-2R).

Waste radex areas: Based upon Radsafe Map, June 1984. Some radex areas may have been consolidated by now.

Tunnel radex areas: Includes tailings, ponds, etc., in and around portals.

Craters: $\quad$ Includes only the area within the crater (based upon approximations from NTS planning maps).

Drill pads at craters: An estimate of the disturbed area around the crater. This estimate was usually made by simply doubling the area of the crater. This is a rough approximation.

Used and unused

drill pads:

These include an estimate of one hectare at each mapped pad.

Estimated area of facilities:

Best guess by Medica and Hunter.

Burned Acres:

Fires since 1978; area estimated by Chief Gudeman, through January 1988.

Roads (Paved):

Measured on maps. Length $X(18.3 \mathrm{~m}$ width $)$.

(Dirt):

Measured on maps. Length $X(6.10 \mathrm{~m}$ width). Many small dirt roads are not on the maps used. Skid lanes are not included. We consider this an underestimate.

Waste Mgt. areas: $\quad$ Estimates by Neagle and Straight. January 1988.

\footnotetext{
All calculations are based upon measurements made from maps, and therefore should be considered crude estimates.

“Allred, D. M., D E. Beck, and C. D. Jorgensen. 1963. Biotic Communities of the Nevada Test Site. Brigham Young University Science Bulletin, Biological Series 2(2). 52 pp.
} 


\title{
SECTION 2 STATUS OF DESERT EPHEMERALS ON THE NEVADA TEST SITE IN 1988
}

by

\author{
Richard B. Hunter
}

\section{INTRODUCTION}

The desert areas of the Nevada Test Site (NTS) produce massive and beautiful displays of winter ephemerals every five to ten years (Went and Westergaard 1949). These floral displays result from germinating rainfall in late September through early November, followed by sufficient spring rainfall for good growth (Beatley 1974). In addition, each year there is a significant population of introduced grasses mixed with small numbers of native ephemerals, which germinate from fall through early spring, and grow a variable amount, depending upon weather conditions.

Winter ephemeral plants start as germinating seeds and produce virtually their entire substance using the resources available during growth. The densities are largely a function of seed reserves and rainfall/temperature conditions. Over the short term, densities are affected by human disturbances such as scraping and fires, which reduce seed reserves; salt, shading, and chemical pollutants, which reduce or enhance germination; and compaction and toxins, which reduce establishment. Following germination and establishment, final individual plant sizes and reproduction are affected by soil moisture; fertility and toxicity; shading; temperature fluctuations; and crowding.

Over a longer time span ephemeral populations are subject to competitive interactions with other species, including differential responses to grazing; disturbance; soil weathering; and minute additions of nutrients through rainfall, nitrogen fixation, and similar natural processes. The greatest in magnitude of these interactions are probably biological ones (Janzen 1986).

Ephemeral populations are, therefore, sensitive to many types of disturbance. One objective of the NTS Basic Environmental Compliance and Monitoring Program (BECAMP) is to determine and understand the changes in the fauna and flora with time. To accomplish this task BECAMP measures ephemeral population characteristics (species composition, densities, and sizes) at sites scattered around the NTS. Some are baseline sites with hurnan disturbances limited as much as possible to widespread airborne pollutants. BECAMP purposely selected a greater number of sample sites that are representative of human (Department of Energy related) disturbances on the NTS. Examples are aboveground blast areas from 1950s tests, subsidence craters, roadsides, construction areas, and areas contaminated by 
radionuclides. A few are disturbed by natural forces such as lightning-initiated fires and gophers.

The wirter of 1987 and spring of 1988 were relatively normal, in that rainfall was limired and sporadic, and temperatures were low. (See Turner and Randall [1989] on correlations of rainfall with ephemeral production.) Some germination of annuals occurred in the warmer areas in early to mid-November 1987, following rainfall in late October varying from 17 to 38 $\mathrm{mm}$. Only sparse rain fell in the best growing season, March-April 1988, however, so plants were small and fruited pooriy, if at all. Variations within that generalization occurred in a normal manner, such that more fertile sites and those with reduced evaporation and transpiration produced larger or more numerous plants.

BECAMP ecology efforts in 1988 were directed toward monitoring the status of ephemerals on five baseline sites, two burned areas, three blast zones of 1950 s atmospheric nuclear bomb tests, a roadside, and a site scraped in 1987 during clean-up operations on a 1950 s era radicactive waste dump.

\section{STUDY SITES AND METHODS}

The following study sites were sampled for ephemerals in 1988: FRFO01, FRF002, JAFO01, MER001, MER002, MID001, PAMO01, RAM001, ROV005, ROV006, YUF001, YUF009, YUF010, YUF011, YUF012, YUF014, YUF015, YUF016, YUF017, YUF018. The Nevada State Coordinates of these locations are given in Appendix. $3 A$, and their approximate locations are shown in Figure 2.1.

Procedures for studying annuals were the same as those used in 1987 (Hunter and Medica 1989). The basic procedure was to harvest all ephemerals from 20 randomly placed $0.025-\mathrm{m}^{2}$ quadrats, arranged along a $50-\mathrm{m}$ steel tape. Species were then identified within a $100-\mathrm{m}^{2}$ areit surrounding the harvested quadrats, and agair from a $1000-\mathrm{m}^{2}$ area surrounding that. All harvested specimens were dried and weighed to determine weight per plant and per square meter. For identification purposes specimens were taken from the 100 - and $1000-\mathrm{m}^{2}$ areas. Those species which were not found in the harvested quadrats were deemed too rare to significantly affect the biomass of ephemerals on a given site.

Deviations from this procedure occurred at several sites. At RAM001 20- $\times 50$ - $\mathrm{mm}$ quadrats were used due to the low density of ephemerals, and at YUMO01 (Yucca Mountain burn) 10$\times 10-\mathrm{cm}$ quadrats were used due to the high density. At YUMO01 and YUFOD9 ten instead of 20 quadrats were harvested because of the low diversity seen at those sites, and at MER001 25 quadrats randomized on a grid were used, following an historical sainpling method.

When an herbaceous perennial or biennial occurred on a plot, the species was censused with ephemerals if it normally appeared alive above ground only in the spring. Bunch grasses 


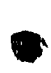

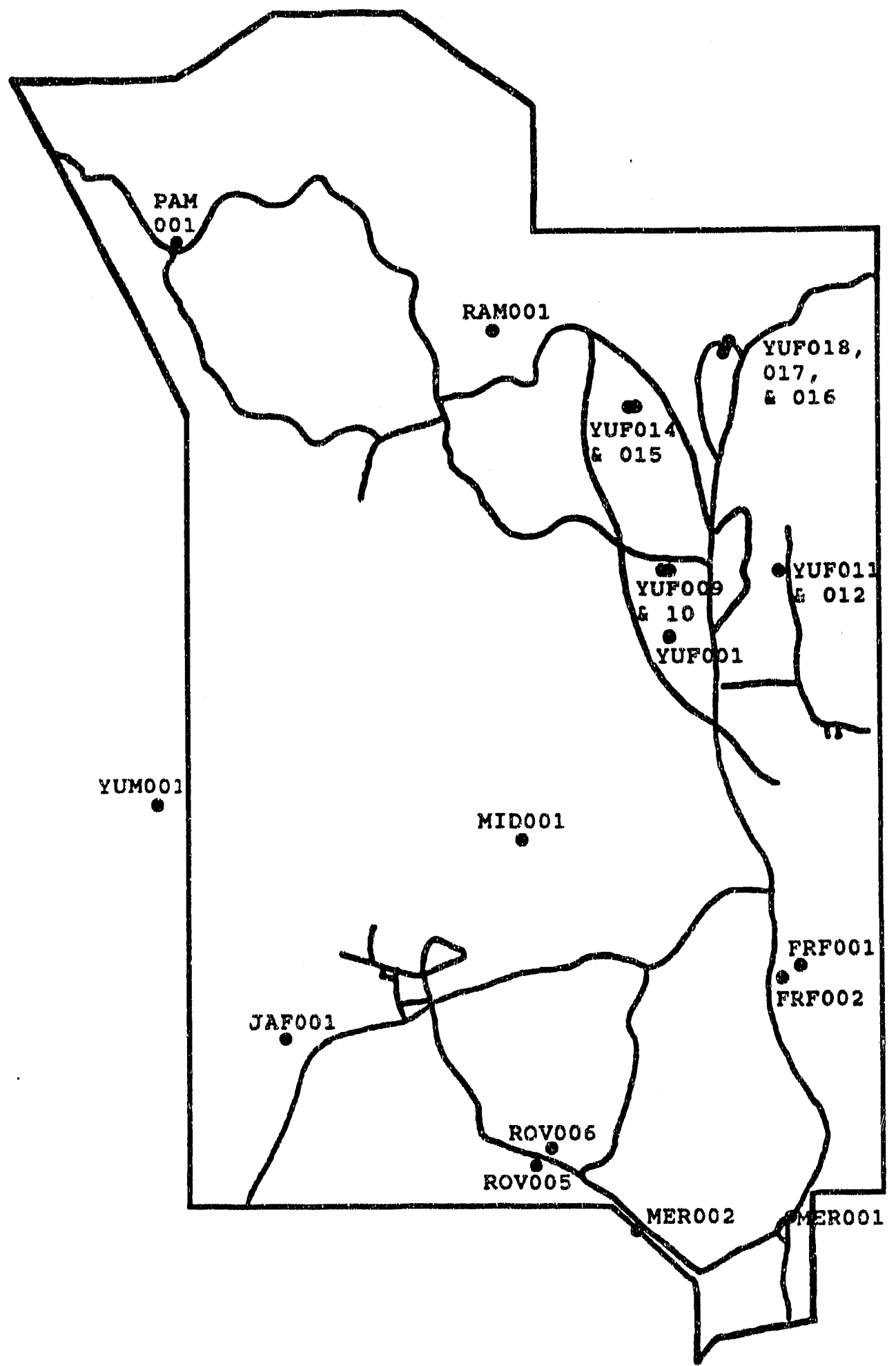

Figure 2.1 Ephemeral plant study sites for 1988 
and other herbaceous perennials were sampled with perennials if they could be conveniently measured during the summer. Summer annial plants were sometimes measured with the perennials on a site, but never harvested. Their presence was noted on perennial data sheets.

Taxonomy of ephemerals followed Munz (1974) and/or Cronquist et al. (1977), with synonymy following Kartesz and Kartesz (1980). Specimens were compared to those in the NTS/DOE herbarium curated by BECAMP personnel, and a voucher for each species collected in 1988 was deposited in that herbarium.

The error terms on density and biomass in Tables 1-26 are standard errors of the mean (sem). The average \pm sem of the total number of plants per quadrat and $\mathrm{g} / \mathrm{m}^{2}$ per quadrat are also given. They were calculated without checking for normal distribution, and should be used with caution when comparing data from different points and times. They are included to give a representation of variability. Species which occurred in only one of twenty quadrats had standard errors equal to the mean, and only the most common species had standard errors less than half the mean.

Soil moisture was measured on Plots MER001a and MER001b using fiberglass-block electrical resistance sensors (Soil Test, Incorporated; Colman and Hendrix 1949) calibrated as described by Hunter and Greger 1986. The calibration technique involved equilibration to a constant reading of a soil and sensor in a closed container with periodic additions of water. Estimation of volumetric soil moisture was based on an assumed soil bulk density of $1 \mathrm{~g} / \mathrm{cc}$ (particles $<2 \mathrm{~mm}$ ), and available soil moisture was estimated as that greater than $5 \%$ moisture.

\section{RESULTS}

Results for the sites examined in 1988 appear in Tables 2.1 though 2.26 for the quadrats for which biomass was sampled. For rarer species presence-absence data are presented in Tables 2.27 through 2.30. The original data sheets are stored in the BECAMP Data Repository, and the data are expected eventually to be in the BECAMP data base managed by the Desert Research Institute (DRI).

Tables 2.1 through 2.26 are presented roughly in order of altitude, beginning with the Mojave Desert valleys and ending on the mesas. On two sites (FRF001, YUF001) replicate transects were done while training personnel to use the technique. I believe the differences between replicates reflect actual variations in ephemeral populations between adjacent sample locations, rather than errors made during training, with the possible exception of the number of rare species recognized (Tables $2.27,2.28$ ).

Estimates of available soil moisture in the top $30 \mathrm{~cm}$ of soil are given in Table 2.31 for Plot MER001a in Mercury, NV. The precipitation reported by NOAA/USWB in the periods between readings of soil moisture sensors is included. 


\section{DISCUSSION}

It appears there are three modes with which man is interacting with annual populations on the Nevada Test Site. The greatest in terms of effect is not the activities of the Department of Energy; it is the introduction of non-native species to the Western United States in years prior to the establishment of the NTS. The second is the interaction between man and range fires, which have burned significant areas on the NTS. Since many range fires are caused by lightning, and all are controlled to some extent by DOE-supported fire-suppression efforts, the primary effert of the DOE proprietorship is probably to minimize the areas affected by burns. The third mode is the scraping of land for NTS construction and cleanup activities. The underground testing of nuclear weapons entails a small amount of land surface clearing for road construction and drilling activities. General support for NTS personnel also includes some clearing activities. In 1988 no observations were made to study the possibility that radiation from previous testing or toxic gas spills from current testing had any effects on germination, growth, or reproduction of ephemeral plants. There is a continuing possibility, not studied, that nitrogen oxides from air pollution might increase fertility of the desert soils, and thus growth of desert ephemerals (Heil et al. 1988).

Other agents affecting ephemeral plants on the NTS include the major influence on their growth - the weather - and also pocket gophers (Thomomys umbrinus) and other small burrowing rodents; granivorous rodents (e.g., Dipodomys merriami) and granivorous birds (e.g., Gambel's quail, chukar); and grazing animals (feral horses, deer, and rabbits).

Introduced species of ephemerals encountered in 1988 are indicated with asterisks in Table 2.27-2.30. Of those species, three are widespread on the NTS (Bromus rubens, $B$. tectorum, and Salsola sp.), and one (Sisymbrium altissimum) is apparently increasing in abundance (compare Tables 2.27-2.29 with Beatley (1965, 1966) and Rickard and Sauer 1982). The fifth, Erodium cicutarium, which was probably introduced into Mexico much earlier than the other species (Frenkel 1970), appears to be at an equilibrium status of being locally abundant on disturbed areas but largely absent from undisturbed sites.

The two abundant Bromus species now dominate most sites where annuals are common on the NTS. As discussed in Hunter and Medica (1989), they are associated with an increased fire frequency, due to the persistence of the dead plants after they die in the late spring. Note that the burned area in Mid Valley (MID001, Table 2.11) was dominated by B. tectorum the second spring after it burned. This suggested that fire was considerably more detrimental to the perennials and native ephemerals than to the Bromus species, and that the adaptation to fire propagation was advantageous to the brome grasses. $E$ tectorum spread through the Pacific Northwest in the late 19th century (Mack 1981) with the introduction of grazing animals. Both B. tectorum and B. rubens were absent from Southern California and the Death Valley area in 1891, when the vegetation was surveyed by the Dept. of Agriculture (Coville, 1893). 
Two sites repeatedly surveyed for Bromus species for which we have past data are plots in Rock Valley censused by the late Dr. Janice Beatley. A comparison of BECAMP dara taken next to those plots (Table 2.32) shows a dramatic increase in the densities of $B$. rubens in Rock Valley. These densities were representative of other Mojave Desert sections of the NTS.

A comparison of annual net production by ephemerals on Beatley Plot 3 with an equation derived by Turner and Randall (1989) shows $36 \pm 8 \mathrm{~g} / \mathrm{m}^{2}$ measured (Table 2.1), versus 26 $\mathrm{g} / \mathrm{m}^{2}$ predicted. For Beatley Plot 4 (Table 2.2), however, measured production was just $14 \pm$ $4 \mathrm{~g} / \mathrm{m}^{2}$, and averaging the quadrat data from the two BECAMP Plots from Rock Valley then closely resemble the predicted values $\left(25 \pm 5 \mathrm{~g} / \mathrm{m}^{2}\right)$. The two Beatley plots represent two fairly different habitats, while Turner and Randall's data were from a wide variety of sites in Rock Valley.

The causes of the dramatic increase in Bromus rubens densities were not clear. Experiments in the late 1960s and mid-1970s showed a marked tendency for B. rubens to increase dramatically when undisturbed desert was irrigated (Medica, personal communication; Romney et al. 1978), and the increase was enhanced significantly by added nitrogen (Romney et al. 1978; Hunter et al. unpublished data). Precipitation (September through March) during the major period of increase, 1975-1988, was $141 \pm 13 \mathrm{~mm}$ (sem), versus $103 \pm 19$ between 1964 and 1974. It is also possible, but purely speculative, that $\mathrm{NO}_{\mathbf{x}}$ pollutants have increased the supply of nitrogen in the upper surface of desert soils and thereby increased growth and reproduction of these weedy species (Heil et al. 1988). These factors might have abetted the increase, but it is also reasonable that these grasses have not yet reached an equilibrium concentration since their introduction to the Mojave Desert in the early twentieth century.

The dense and heavy populations of $B$. rubens on the gopher area in Mercury Valley (MER002, Table 2.4) are an extreme population related to gopher removal of shrubs and soil disturbance associated with their burrowing activities. A similar situation was sampled in Frenchman Flat in 1987 (Hunter and Medica, 1989), with densities of $2644 \pm 716$ and biomass of $37 \pm 8 \mathrm{~g} / \mathrm{m}^{2}$. The 1987 site was also an area with considerable gopher activity and reduced shrub densities.

Historical data are less available for Bromus tectorum (as opposed to Bromus rubens) on the NTS, but it appears to be increasing dramatically in abundance in some areas. Rickard and Sauer (1982), for example, found only B. rubens at the T2C site of the Shasta test in the northwestern part of Yucca Flat in 1957. It was absent from the area around Sedan crater in censuses of 1962, 1965, and 1975-6, appearing only in censuses of 1983 and 1986 (Hunter, unpublished data). In 1988 it was abundant around Sedan crater, most dramatically in the areas bared by the Sedan blast (YUF016, YUF017; Tables 2.21, 2.22), where it dominated the vegetation. In the area undisturbed by the Sedan event (YUF018, Table 2.23) B. rubens was the most common ephemeral.

A similar pattern occurred at the T1 ground zero. The blast area, in which only sparse perennials have reappeared, was dominated in 1988 by a mixed population of $B$. tectorum and 
B. rubens (YUF009, Table 2.15). In contrast, a control plot just outside the tlast area was dominated by B. rubens alone (YUF010, Table 2.16). A photograph of a revegetation site within the T1 blast area taken in 1983 (Figure 2.2) shows a good population of B. rubens with a few specimens of $B$. tectorum. In the interim it appears that Bromus tectorum increased significantly while $B$. rubens suffered from the competition. A comparison of information on the two Bromus species when they occurred together suggests some differences (Table 2.33). First, there was a tendency in disturbed areas for Bromus rubens to be smaller than in a shrub-dominated microhabitat. There was an opposite tendency in Bromus tectorum. It appeared, then, that disturbance favored the larger species, B. tectorum, while the absence of disturbance favored $B$. rubens. Thus a pattern appeared in which $B$. tectorum was dominant on roadsides and disturbed areas, while $B$. rubens was dominant within a shrub community.

As noted above, the dominance of the ephemeral flora on the NTS by the Bromus species is a relatively new phenomenon. The ecological consequences are therefore just beginning to be seen. One consequence noted in other areas is an increase in the extent of fires. To determine what densities of grass were needed to carry a fire, a sample was taken at a site within an area that burned in June 1988, in a valley between Forty Mile Canyon and Yucca Mountain. The area sampled was protected from the fire by a knoll and a dirt road, (Figure 2.3), and was very likely representative of the vegetation which had burned. The average densities were $1460 \pm 380$ plants per square meter of $B$. rubens and $700 \pm 40$ of $B$. tectorum. Total biomass of dead grass was $47 \pm 13 \mathrm{~g} / \mathrm{m}^{2}$. Comparison of these data with those from areas sampled earlier in the spring suggested several areas would easily burn, if ignited. In particular, the Mid Valley burned area (Table 2.11) and the Mercury Valley gopher area (Table 2.11) should have had enough dry grass to carry a fire. These conditions appeared to be common on the N"TS in 1988, and it seemed reasonable that the absence of range fires in most areas was not due to lack of fuel, but rather to the absence of factors causing ignition. A record of fire causes from 1978 to the present is maintained by the NTS fire protection services, and their data suggest most fires are caused by lightning and cigarettes thrown out of car windows, with a few ignited by tests of ammunition.

One area which has burned frequently in the history of the test site is Mid Valley. As noted above, the presence of heavy concentrations of B. tectorum on the site burned in 1986 (MID001b, Table 2.11) suggested that the fires there have favored that species.

Another plant species which may be adapted to fire is the Joshua Tree, Yucca brevifolia. By sticking up above the level of surrounding vegetation, it seems to attract lightning, while the long persistence of dried leaves along its stems makes the plant easily burred. As we showed in the reports of 1987 studies (Hunter and Medica 1989; Table 10, Figure 5), many $Y$. brevifolia of intermediate size produced new shoots at the base following a fire.

Another significant question relating to the Bromus species is whether they are competitively excluding the native desert "wildflowers" from their historic habitat by their dense growth. However, the nature of ephemerals in the Mojave Desert seems to be an adaptation to 


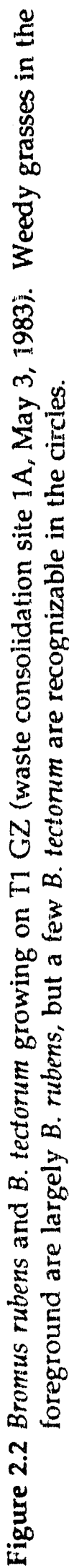

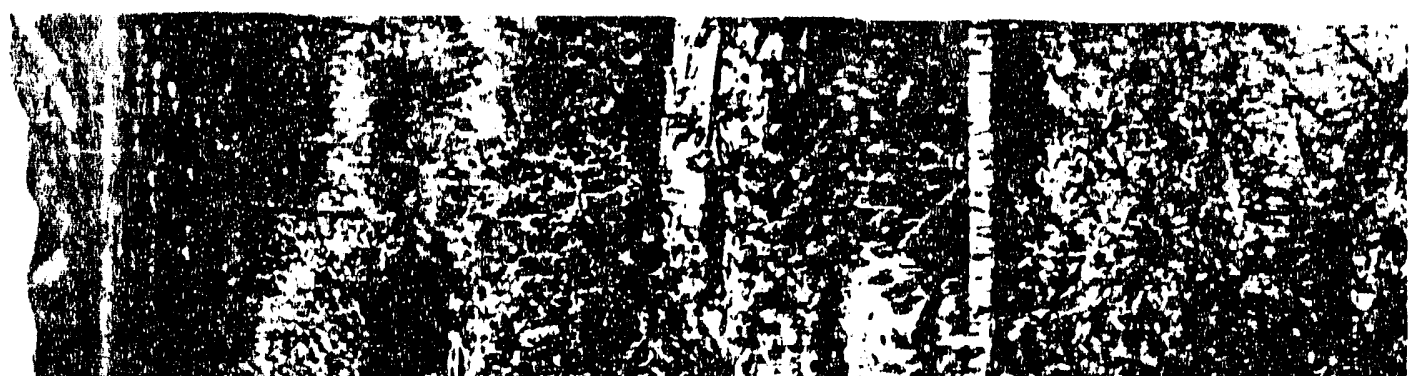

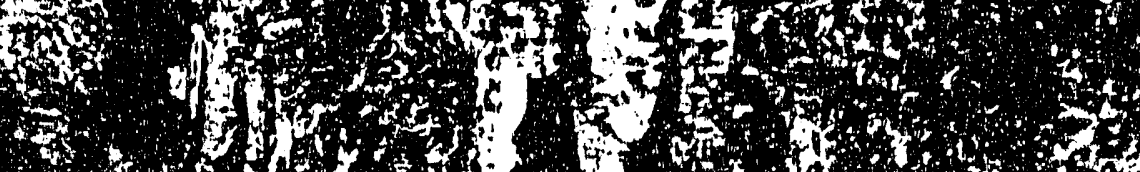

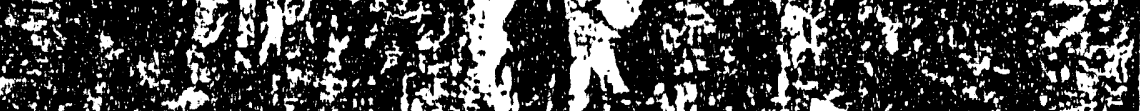

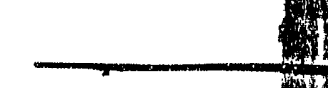

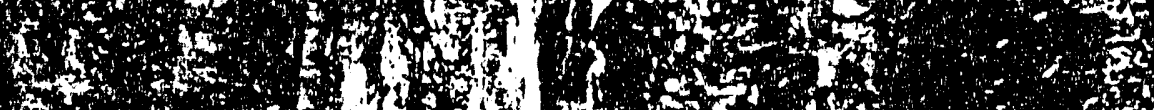

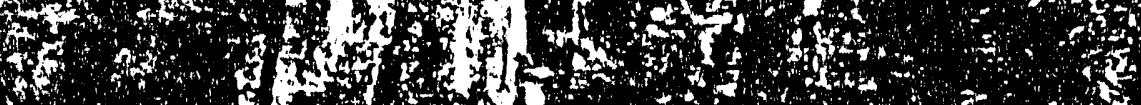

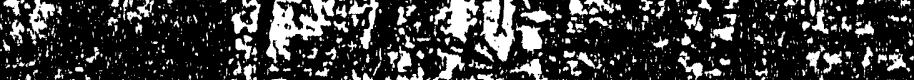

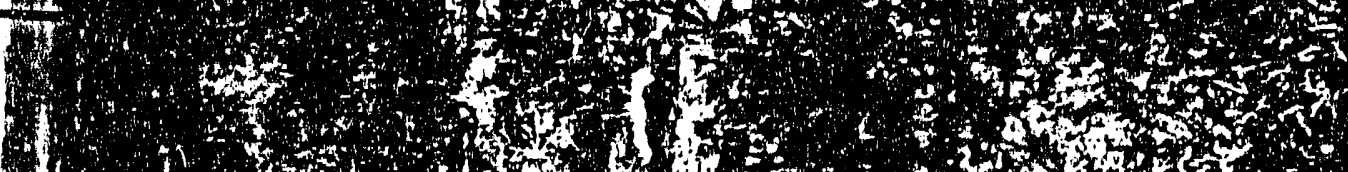

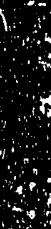

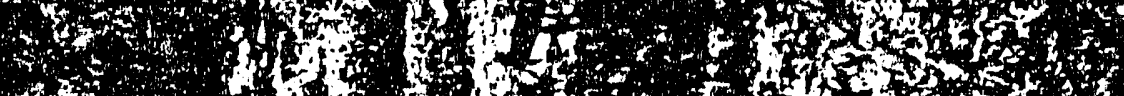

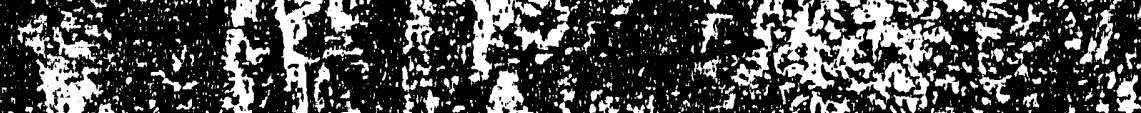

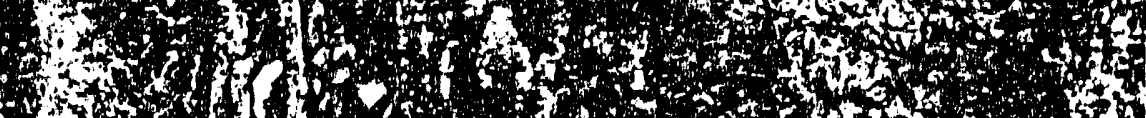

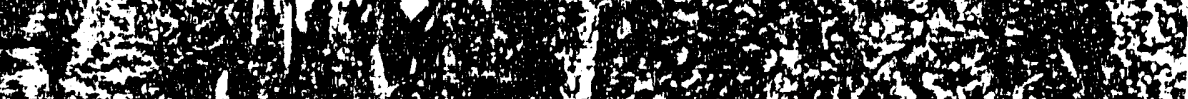

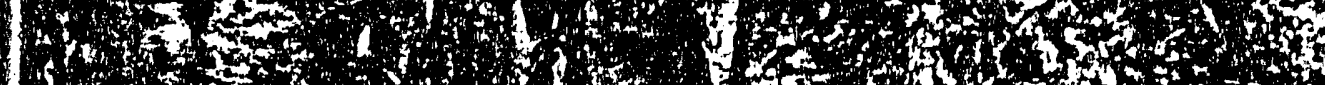

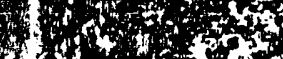

$40,4+5$

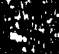

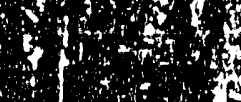

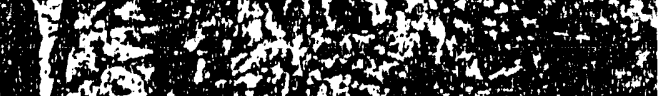

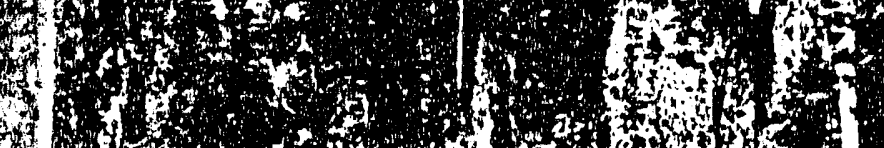

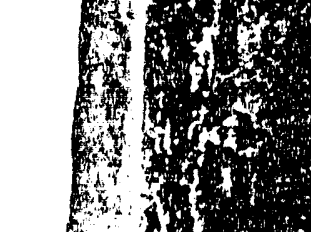
$+4$ oryons (1) 3. $13,2,+4$

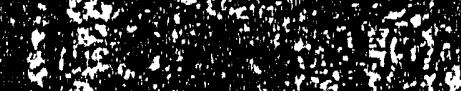

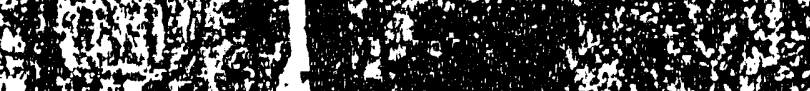

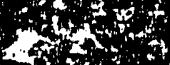
10150

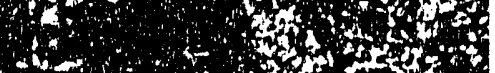

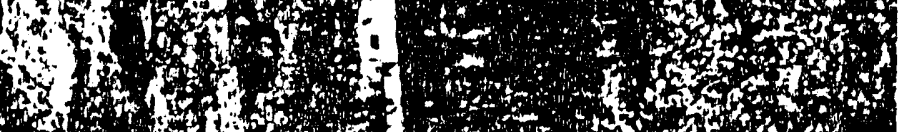

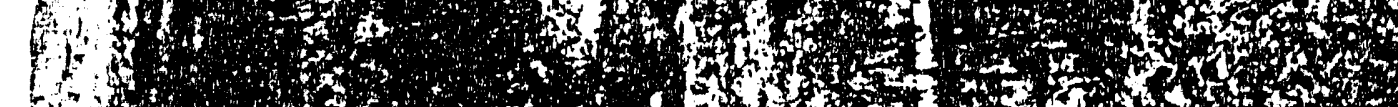

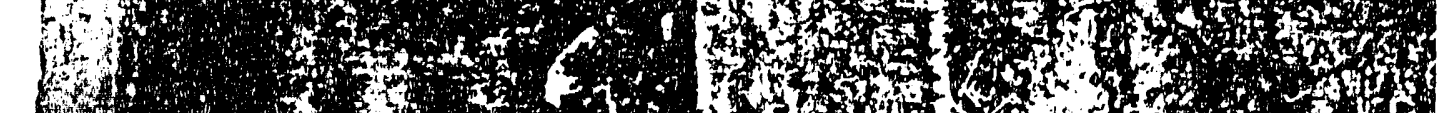

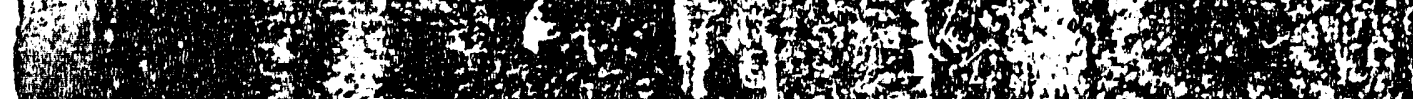

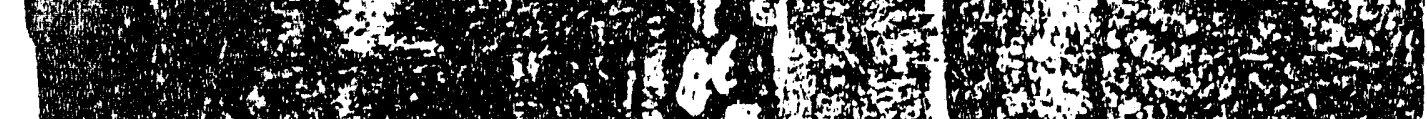

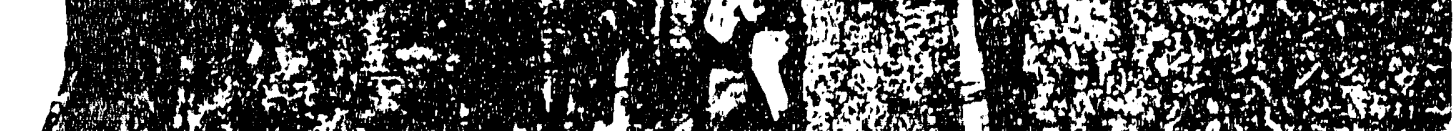

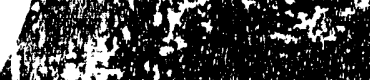
10. $3 x+4$ $3(x+2)$

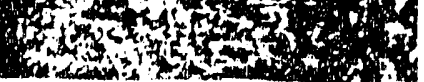


massive germination and growth only in rare years, during which many species grow and reproduce much better than normal. Those events have occurred on the NTS in 1958, 1966, $1968,1969,1973$, and 1978, but not in the 1980 s. They require a wet fall as well as a wet spring (Beatley 1974). It is probable that the native ephemerals growing in "normal" dry years are not part of the main, successfully adapted populations of their respective species; therefore, comparisons of diversities in "normal" years would be misleading. Available data are probably not yet sufficient to document a decrease in diversity which might be caused by the introduced species. Some experimental work elucidating the nature of the nativeintroduced species interactions is probably warranted to answer this question.

The third major introduced species which continues to be of significance on the NTS is the tumbleweed, Russian thistle, Salsola sp. The taxonomy of this species is problematic, and we have therefore not given it a specific epithet in this report. Dr. Beatley used S. paulsenii and $S$. iberica to describe two intergrading morphological types present on the NTS (Beatley 1973; Wallace and Romney 1972, p. 152). It invaded the "ground zeros(GZ)" shortly after the atmospheric testing in the 1950s (Shields et al. 1963), and was still dominant on some of those sites until the late 1970s. It now occurs in abundance on disturbed areas in the year following the disturbance, as was seen in 1988 on one site studied, Waste Management Consolidation Site 3B (Table 2.17).

Russian thistle germinates in the winter or spring, but grows in the summer. The population density on XUF011 in April 1988 was $12 \pm 5 / \mathrm{m}^{2}$ and biomass was $0.7 \mathrm{~g} / \mathrm{m}^{2}$. In August the site had a Russian thistle density still $14 \pm 2 / \mathrm{m}^{2}$ with biomass of $334 \mathrm{~g} / \mathrm{m}^{2}$, showing a great deal of growth and essentially no occurrence of mortality. This cannot be taken as a representative productivity of Russian thistle for other sites where many seedlings germinated, because in other locations it appeared the seedlings died rather than grew, as at T1 GZ (YUF009, Table 2.15) and Sedan (YUF016, Table 2.21) and T2 GZ (YUF014).

At the present time it seems likely that a successional process is occurring on the "ground zeros" that is very similar to a sequence described by Piemeisel for farmland in Idaho (Piemeisel 1938, 1951). Over a period of several years he documented first invasion by Salsola, then its replacement by Bromus species; Bromus was itself replaced by the mustards Sisymbrium altissimum and a Descurainia sp. On the NTS the first step of that process apparently required about 20 to 25 years, and we are now in the Bromus-dominated phase. Sisymbrium altissimum appears now to be increasing in abundance but is not yet really common (Table 2.29).

Some of the introduced species are adapted to growing on disturbed sites, in particular Salsola, Bromus tectorum, and Sisymbrium altissimum. Nevertheless, because Bromus rubens is adapted to a shrub-dominated environment, the introduced species make up a large proportion of the ephemeral biomass even on undisturbed areas (Table 2.34). One cannot therefore suggest that the environmental effects of the introduced species might be mitigated by limiting the extent of disturbance. In 1988 the only introduced plant species with a significant population in general habitats was Bromus rubens. 
Historical data from Rock Valley (Table 2.35) suggest that though Bromus rubens has greatly increased in density, the native annuals have not at the same time decreased in density.

One must bear in mind always that the native species may only be represented accurately in unusual years when the majority of seeds might germinate, but at least we may judge the trend as suggesting that the increase in the introduced species has not caused a decrease in the native species.

Other introduced species may interact with desert ephemerals. A tree, Tamarix ramosissima, is present on the NTS and has caused problems for aquatic ecosystems in Death Valley. Introduced burros and horses are also degrading environments in other areas of Nevada, and may particularly affect small herbaceous plant species like the desert ephemerals by grazing and trampling.

The native ephemerals censused in 1988 included many more species than seen in 1987 . We believe this was an artifact of the increased sampling effort in the second year of the BECAMP monitoring program. In 1987 forty-four species were seen on 4 sites, and in 1988, 113 species were found on 23 sites. Not only was the number of sites increased, but also the range of sampled environments was much greater in 1988 than in 1987.

Table 2.1 Ephemeral population characteristics on Plot ROV005, Beatley Plot 3 in Rock Valley, measured April 11, 1988.

\begin{tabular}{lccc}
\hline Species & $\mathrm{n} / \mathrm{m}^{2}$ & $\mathrm{~g} / \mathrm{m}^{2}$ & $\mathrm{mg} /$ plant \\
\hline Bromus rubens & $2034 \pm 632$ & $34 \pm 8$ & $24 \pm 3$ \\
B. trinii & $28 \pm 24$ & $1 \pm 1$ & $74 \pm 70$ \\
Camissonia claviformis & $2 \pm 2$ & $.02 \pm .02$ & 10 \\
Chaenactis stevioides & $2 \pm 2$ & $.05 \pm .05$ & 25 \\
Descurainia pinnata & $2 \pm 2$ & $.01 \pm .01$ & 5 \\
Eriogonum glandulosum & $10 \pm 8$ & $.11 \pm .09$ & $26 \pm 16$ \\
Eriophyllum pringlei & $16 \pm 14$ & $.17 \pm .12$ & $31 \pm 27$ \\
Linanthus demissus & $2 \pm 2$ & $.004 \pm .002$ & 2 \\
Pectocarya platycarpa & $4 \pm 4$ & $.1 \pm .1$ & 38 \\
Phacelia fremontii & $10 \pm 10$ & $.03 \pm .03$ & 13 \\
Streptanthella longirostris & $6 \pm 4$ & $.13 \pm .11$ & $30 \pm 23$ \\
Vulpia octoflora & $32 \pm 11$ & $.3 \pm .1$ & $10 \pm 2$ \\
& $2140 \pm 630$ & $36 \pm 8$ & \\
Mean Quadrat Totals & & & \\
\hline
\end{tabular}


Table 2.2 Ephemeral population characteristics on Plot ROV006, Beatley Plot 4 in Rock Valley, measured on March 29, 1988.

\begin{tabular}{lrcc}
\hline Species & $\mathrm{n} / \mathrm{m}^{2}$ & $\mathrm{~g} / \mathrm{m}^{2}$ & $\mathrm{mg} / \mathrm{plant}$ \\
\hline Bromus rubens & $514 \pm 158$ & $9 \pm 2$ & $24 \pm 4$ \\
Chaenactis fremontii & $171 \pm 34$ & $4 \pm 2$ & $145 \pm 100$ \\
Cryptantha circumscissa & $2 \pm 2$ & $.002 \pm .002$ & 1 \\
C. micrantha & $38 \pm 12$ & $.22 \pm .08$ & $6 \pm 1$ \\
Descurainia pinnata & $2 \pm 2$ & $.01 \pm .01$ & 4 \\
Eriogonum maculatum & $2 \pm 2$ & $.05 \pm .05$ & 23 \\
Eriophyllum pringlei & $30 \pm 10$ & $.41 \pm .27$ & $10 \pm 4$ \\
Gilia transmontana & $2 \pm 2$ & $.01 \pm .01$ & 5 \\
Malacothrix glabrata & $2 \pm 2$ & $.01 \pm .01$ & 5 \\
Monoptilon bellidiforme & $4 \pm 3$ & $.010 \pm .007$ & $2 \pm 1$ \\
Nama demissum & $2 \pm 2$ & $.01 \pm .01$ & 7 \\
Rafinesquia neomexicana & $2 \pm 2$ & $.14 \pm .14$ & 68 \\
Vulpia octoflora & $2 \pm 2$ & $.3 \pm .1$ & 3 \\
Mean Quadrat Totals & $662 \pm 175$ & $14 \pm 4$ &
\end{tabular}

Table 2.3 Ephemeral population characteristics on Plot JAF001, Jackass Flats baseline plot, measured March 28, 1988.

\begin{tabular}{lrcc}
\hline Species & $\mathrm{n} / \mathrm{m}^{2}$ & $\mathrm{~g} / \mathrm{m}^{2}$ & $\mathrm{mg} /$ plant \\
\hline Astragalus didymocarpus & $2 \pm 2$ & $.04 \pm .04$ & 18 \\
Bromus rubens & $16 \pm 14$ & $1 \pm 1$ & $36 \pm 35$ \\
Cryptantha angustifolia & $6 \pm 4$ & $.08 \pm .07$ & $13 \pm 3$ \\
C. micrantha & $66 \pm 15$ & $.20 \pm .05$ & $4 \pm 1$ \\
Eriogonum maculatum & $4 \pm 3$ & $.02 \pm .01$ & $5 \pm 2$ \\
Mulacothrix glabrata & $4 \pm 3$ & $.03 \pm .03$ & $8 \pm 7$ \\
Streptanthella longirostris & $10 \pm 5$ & $.2 \pm .1$ & $12 \pm 2$ \\
Vulpia octoflora & $854 \pm 103$ & $8.2 \pm 0.8$ & \\
Mean Quadrat Totals & $962 \pm 109$ & $10 \pm 1$ & \\
\end{tabular}


Table 2.4 Ephemeral population characteristics on Plot MER002, Mercury gopher area, measured April 19, 1988.

\begin{tabular}{lccc}
\hline Species & $\mathrm{n} / \mathrm{m}^{2}$ & $\mathrm{~g} / \mathrm{m}^{2}$ & $\mathrm{mg} / \mathrm{plant}$ \\
\hline Amsinckia tessellata & $4 \pm 4$ & $.2 \pm .2$ & 42 \\
Bromus rubens & $3550 \pm 366$ & $70 \pm 6$ & $24 \pm 3$ \\
Chaenactis fremontii & $14 \pm 8$ & $.2 \pm .1$ & $23 \pm 9$ \\
Eriogonum (unidentifiable) & $8 \pm 5$ & $.006 \pm .003$ & $.8 \pm .2$ \\
Euphorbia albomarginata & $106 \pm 32$ & $1.4 \pm .4$ & $12 \pm 6$ \\
Gilia transmontana & $4 \pm 4$ & $.006 \pm .006$ & 3 \\
Vulpia octoflora & $6 \pm 6$ & $.04 \pm .04$ & 21 \\
& $3692 \pm 369$ & $72 \pm 6$ & \\
Mean Quadrat Totals & & & \\
\hline
\end{tabular}

Table 2.5 Ephemeral population characteristics for Plot MER001a, Mercury water balance plot control, measuriu April 7, 1988.

\begin{tabular}{lccc}
\hline Species & $\mathrm{n} / \mathrm{m}^{2}$ & $\mathrm{~g} / \mathrm{m}^{2}$ & $\mathrm{mg} / \mathrm{plant}$ \\
\hline & $43 \pm 130$ & $9 \pm 2$ & $25 \pm 4$ \\
Bromus rubens & $2 \pm 2$ & $.04 \pm .04$ & 24 \\
B. tectorum & $3 \pm 2$ & $.07 \pm .06$ & $22 \pm 12$ \\
Chorizanthe rigida & $2 \pm 2$ & $.02 \pm .02$ & 15 \\
Cryptantha nevadensis & $3 \pm 2$ & $.02 \pm .01$ & 6 \\
Descurainia pinnata & $2 \pm 2$ & $.02 \pm .02$ & 11 \\
Eriogonum deflexum & $6 \pm 4$ & $.34 \pm .26$ & $44 \pm 19$ \\
Erodium cicutarium & $10 \pm 7$ & $.20 \pm .14$ & $20 \pm 8$ \\
Gilia transmontana & $24 \pm 13$ & $.24 \pm .14$ & $9 \pm 2$ \\
Ipomopsis polycladon & $2 \pm 2$ & $.02 \pm .02$ & $9 \pm 3$ \\
Langloisia setosissima & $85 \pm 32$ & $.5 \pm .2$ & \\
Vulpia actoflora & $581 \pm 153$ & $10 \pm 2$ & \\
Mean Quadrat Totals & & & \\
& & & \\
\hline
\end{tabular}


Table 2.6 Ephemeral population characteristics on Pict MER001b, Mercury water balance plot with shrubs removed, measured April 5, 1988.

\begin{tabular}{lccc}
\hline Species & $\mathrm{n} / \mathrm{m}^{2}$ & $\mathrm{~g} / \mathrm{m}^{2}$ & $\mathrm{mg} / \mathrm{plant}$ \\
\hline Bromus rubens & & $16 \pm 5$ & $12 \pm 2$ \\
B. tectorum & $1912 \pm 475$ & $.34 \pm .26$ & $52 \pm 14$ \\
Chaenactis stevioides & $8 \pm 6$ & $3 \pm .2$ & $18 \pm 8$ \\
Chorizanthe rigida & $29 \pm 14$ & $.04 \pm .03$ & $8 \pm 2$ \\
Cryptantha circumscissa & $6 \pm 5$ & $.6 \pm .5$ & $13 \pm 2$ \\
C. recurcata & $54 \pm 42$ & $.07 \pm .07$ & 45 \\
Descurainia pinnata & $2 \pm 2$ & $.09 \pm .07$ & $6 \pm 4$ \\
Eriastrum eremicum & $14 \pm 7$ & $.05 \pm .04$ & $15 \pm 11$ \\
Eriogonum deflexum & $5 \pm 4$ & $.3 \pm .1$ & $5 \pm 2$ \\
Erodium cicutarium & $72 \pm 17$ & $10 \pm 1$ & $47 \pm 7$ \\
Gilia transmontana & $278 \pm 42$ & $.003 \pm .003$ & 2 \\
Ipomopsis polycladon & $2 \pm 2$ & $.19 \pm .06$ & $8 \pm 1$ \\
Langloisia setosissima & $30 \pm 12$ & $.09 \pm .07$ & $30 \pm 1$ \\
Linanthus demissus & $3 \pm 2$ & $.002 \pm .002$ & 1 \\
Pectocarya platycarpa & $2 \pm 2$ & $.09 \pm .06$ & $18 \pm 10$ \\
Schismus sp. & $6 \pm 4$ & $1.7 \pm 0.8$ & $4.4 \pm 0.7$ \\
Vulpia octoflora & $294 \pm 103$ & $1.5 \pm 0.2$ & $6 \pm 2$ \\
Mean Quadrat Totals & $365 \pm 78$ & $32 \pm 6$ & \\
& $3070 \pm 522$ & & \\
\hline
\end{tabular}


Table 2.7 Ephemeral population characteristics on Plot FRF001a, Frenchman Flat baseline plot (Beatley Plot 23), replicate 1, measured April 13, 1988.

\begin{tabular}{lrrr}
\hline Species & $\mathrm{n} / \mathrm{m}^{2}$ & $\mathrm{~g} / \mathrm{m}^{2}$ & $\mathrm{mg} /$ plant \\
\hline Amsinckia tessellata & $4 \pm 4$ & $2 \pm 2$ & 402 \\
Bromus rubens & $258 \pm 153$ & $13 \pm 6$ & $145 \pm 114$ \\
B. tectorum & $4 \pm 4$ & $.2 \pm .2$ & 39 \\
Chaenactis fremontii & $8 \pm 6$ & $.7 \pm .6$ & $92 \pm 62$ \\
Cryptantha circumscissa & $16 \pm 9$ & $.4 \pm .2$ & $30 \pm 12$ \\
C. micrantha & $18 \pm 6$ & $.4 \pm .1$ & $19 \pm 2$ \\
C. recurvata & $2 \pm 2$ & $.03 \pm .03$ & 15 \\
Descurainia pinnata & $12 \pm 12$ & $2 \pm 2$ & 134 \\
Eriophyllum pringlei & $4 \pm 4$ & $.08 \pm .08$ & 20 \\
Gilia sinuata & $16 \pm 6$ & $.3 \pm .1$ & $20 \pm 6$ \\
Malacothrix glabrata & $4 \pm 3$ & $13 \pm 13$ & $3172 \pm 3152$ \\
Monoptilon bellidiforme & $2 \pm 2$ & $.05 \pm .05$ & 25 \\
& $348 \pm 156$ & $31 \pm 17$ & \\
Mean Quadrat Totals & & & \\
\hline
\end{tabular}

Table 2.8 Ephemeral population characteristics on Plot FRF001, replicate 2, measured April 13, 1988.

\begin{tabular}{lrrr}
\hline Species & $\mathrm{n} / \mathrm{m}^{2}$ & $\mathrm{~g} / \mathrm{m}^{2}$ & $\mathrm{mg} / \mathrm{plant}$ \\
\hline & $152 \pm 74$ & $5 \pm 2$ & $50 \pm 11$ \\
Bromus rubens & $2 \pm 2$ & $2 \pm 2$ & 748 \\
Chaenactis fremontii & $6 \pm 4$ & $.05 \pm .04$ & $8 \pm 1$ \\
Cryptantha circumscissa & $36 \pm 15$ & $.6 \pm .2$ & $20 \pm 5$ \\
C. micrantha & $2 \pm 2$ & $.05 \pm .05$ & 24 \\
C. recurvata & $2 \pm 2$ & $3 \pm 3$ & 1560 \\
C. pterocarya & $12 \pm 5$ & $.5 \pm .3$ & $46 \pm 24$ \\
Descurainia pinnata & $2 \pm 2$ & $.2 \pm .2$ & 82 \\
Eriogonum maculatum & $6 \pm 3$ & $.19 \pm .11$ & $32 \pm 3$ \\
Eriophyllum pringlei & $4 \pm 3$ & $.08 \pm .05$ & $20 \pm 2$ \\
Gilia sinuata & $8 \pm 5$ & $.7 \pm .5$ & $113 \pm 67$ \\
Malacothrix glabrata & $2 \pm 2$ & $.2 \pm .2$ & 82 \\
Mentzelia albicaulis & $2 \pm 2$ & $.05 \pm .05$ & 24 \\
Monoptilon bellidiforme & $238 \pm 80$ & $12 \pm 5$ & \\
Mean Quadrat Totals & & & \\
\hline
\end{tabular}


Table 2.9 Ephemeral population characteristics on Plot FKFO02a, Frenchman Flat roadside, measured on March 30, 1988.

\begin{tabular}{lrcc}
\hline Species & $\mathrm{n} / \mathrm{m}^{2}$ & $\mathrm{~g} / \mathrm{rn}^{2}$ & $\mathrm{mg} / \mathrm{plant}$ \\
\hline Bromus nubens & $4 \pm 3$ & $.2 \pm .1$ & $53 \pm 5$ \\
Chorizanthe rigida & $2 \pm 2$ & $.01 \pm .01$ & 5 \\
Cryptantha micrantha & $88 \pm 25$ & $2.0 \pm 0.8$ & $20 \pm 8$ \\
Cryptantha recuriata & $8 \pm 6$ & $.09 \pm .07$ & $10 \pm 2$ \\
Eriogonum (unidentifiable) & $4 \pm 3$ & $.06 \pm .06$ & $16 \pm 14$ \\
Eriopinyllum pringlei & $2 \pm 2$ & $.03 \pm .03$ & 16 \\
Erodium cicutarium & $2 \pm 2$ & $.02 \pm .02$ & 101 \\
Gilia cana & $2 \pm 2$ & $.04 \pm .04$ & 19 \\
Linanthus arenicola & $2 \pm 2$ & $.002 \pm .002$ & 1 \\
Nama demissum & $24 \pm 8$ & $.3 \pm .1$ & $16 \pm 6$ \\
Schismus sp. & $2 \pm 2$ & $.03 \pm .03$ & 16 \\
Mean Quadrat Totals & $210 \pm 78$ & $5 \pm 2$ & \\
& & & \\
\hline
\end{tabular}


Table 2.10 Ephemeral population characteristics on Plot FRF002b, Frenchman Flat roadside control, measured on March 30, 1988.

\begin{tabular}{|c|c|c|c|}
\hline Species & $\mathrm{n} / \mathrm{m}^{2}$ & $\mathrm{~g} / \mathrm{m}^{2}$ & $\mathrm{mg} /$ plant \\
\hline Amsinckia tessellata & $8 \pm 4$ & $1.7 \pm 1.5$ & $228 \pm 166$ \\
\hline Astragalus acutirostris & $2 \pm 2$ & $.07 \pm .07$ & 37 \\
\hline Bromus rubens & $34 \pm 22$ & $2.5 \pm 1.6$ & $116 \pm 33$ \\
\hline Camissonia pterosperma & $8 \pm 4$ & $.03 \pm .02$ & $4 \pm 1$ \\
\hline Chaenactis stevioides & $38 \pm 25$ & $.85 \pm .69$ & $15 \pm 9$ \\
\hline Chaenactis xantiana & $2 \pm 2$ & $.06 \pm .06$ & 33 \\
\hline Cryptantha circumscissa & $2 \pm 2$ & $.02 \pm .02$ & 12 \\
\hline C. pterocaryaa & $11 \pm 8$ & $.14 \pm .10$ & $12 \pm 1$ \\
\hline Descurainia pinnata & $131 \pm 92$ & $3.5 \pm 2.0$ & $93 \pm 35$ \\
\hline Eriogonum maculatum & $2 \pm 2$ & $.03 \pm .03$ & $14^{-}$ \\
\hline Eriophyllum pringlei & $21 \pm 17$ & $.07 \pm .05$ & $5 \pm 3$ \\
\hline Gilia sinuata & $15 \pm 7$ & $.17 \pm .08$ & $14 \pm 5$ \\
\hline Langloisia setosissima & $2 \pm 2$ & $.002 \pm .002$ & 1 \\
\hline Lepidium lasiocarpum & $46 \pm 46$ & $1.5 \pm 1.5$ & 34 \\
\hline Linanthus arenicola & $4 \pm 4$ & $.006 \pm .006$ & 2 \\
\hline Lupinus flavoculatus & $2 \pm 2$ & $.01 \pm .01$ & 5 \\
\hline Malacothrix glabrata & $17 \pm 8$ & $1.0 \pm 0.6$ & $50 \pm 17$ \\
\hline Mentzelia albicaulis & $8 \pm 6$ & $.7 \pm .5$ & $88 \pm 1$ \\
\hline Nama demissum & $4 \pm 4$ & $.004 \pm .003$ & $1 \pm 0$ \\
\hline Phacelia fremontii & $2 \pm 2$ & $.01 \pm .01$ & 7 \\
\hline P. vallis-mortae & $2 \pm 2$ & $.4 \pm .4$ & 235 \\
\hline Rafinesquia neomexicana & $2 \pm 2$ & $.2 \pm .2$ & 103 \\
\hline Streptanthella longirostris & $2 \pm 2$ & $.04 \pm .04$ & 22 \\
\hline Vulpia octoflora & $11 \pm 11$ & $.16 \pm .16$ & 14 \\
\hline Mean Quadrat Totals & $375 \pm 151$ & $13 \pm 5$ & \\
\hline
\end{tabular}


Table 2.11 Ephemeral population characteristics from Plot MID001a, Mid Valley burned area, measured on May 5, 1988. (Herbaceous perennials are not included in the totals.)

\begin{tabular}{lccc}
\hline Species & $\mathrm{n} / \mathrm{m}^{2}$ & $\mathrm{~g} / \mathrm{m}^{2}$ & $\mathrm{mg} / \mathrm{plant}$ \\
\hline Allium nevadense & $2 \pm 2$ & $.008 \pm .008$ & 4 \\
Bromus rubens & $114 \pm 37$ & $1.7 \pm 0.7$ & $26 \pm 11$ \\
B. tectorum & $3916 \pm 752$ & $89 \pm 16$ & $33 \pm 7$ \\
Chaenactis stevioides & $38 \pm 25$ & $.85 \pm .69$ & $15 \pm 9$ \\
Chaenactis xantiana & $2 \pm 2$ & $.06 \pm .06$ & 33 \\
Cryptantha micrantha & $6 \pm 6$ & $.002 \pm .002$ & $<1$ \\
Descurainia pinnata & $24 \pm 13$ & $.11 \pm .08$ & $3 \pm 1$ \\
Eriastrum eremicum & $32 \pm 32$ & $.09 \pm .09$ & 29 \\
Gilia sinuata & $44 \pm 25$ & $.29 \pm .13$ & $11 \pm 7$ \\
Gilia transmontana & $44 \pm 42$ & $.25 \pm .19$ & $19 \pm 14$ \\
(Phlox stansburyi) & $(86 \pm 19)$ & $(16 \pm 4)$ & $(231 \pm 57)$ \\
Salsola spp. & $2 \pm 2$ & $.008 \pm .008$ & 4 \\
& & & \\
Mean Quadrat Totals & $4182 \pm 768$ & $92 \pm 17$ & \\
& & & \\
\hline
\end{tabular}


Table 2.12 Ephemeral population characteristics on Plot MID001b, Mid Valley control area, May 5, 1988. (Herbaceous perennials are not included in the totals.)

\begin{tabular}{lccc}
\hline Species & $\mathrm{n} / \mathrm{m}^{2}$ & $\mathrm{~g} / \mathrm{m}^{2}$ & $\mathrm{mg} / \mathrm{plant}$ \\
\hline Astragalus purshii & $2 \pm 2$ & $.02 \pm .02$ & 12 \\
Bromus rubens & $362 \pm 84$ & $4.3 \pm 1.2$ & $11 \pm 1$ \\
B. tectorum & $414 \pm 152$ & $8 \pm 3$ & $20 \pm 2$ \\
Bromus unidentifiable & $100 \pm 39$ & $.7 \pm .4$ & $6 \pm 1$ \\
Chorizanthe thurberi & $6 \pm 3$ & $.02 \pm .02$ & $4 \pm 3$ \\
Cryptantha circumscissa & $16 \pm 11$ & $.03 \pm .02$ & $2 \pm 0$ \\
C. pterocarya & $30 \pm 20$ & $.17 \pm .11$ & $6 \pm 1$ \\
Descurainia pinnata & $4 \pm 4$ & $.002 \pm .002$ & $<1$ \\
Eriastrum eremicum & $6 \pm 3$ & $.03 \pm .02$ & $5 \pm 2$ \\
Erodium cicutarium & $2 \pm 2$ & $.05 \pm .05$ & 27 \\
Euphorbia albomarginata & $4 \pm 4$ & $.01 \pm .01$ & 6 \\
Gilia sinuata & $20 \pm 10$ & $.07 \pm .04$ & $4 \pm 1$ \\
Gilia transmontana & $2 \pm 2$ & $.03 \pm .03$ & 13 \\
(Hilaria jamesii) & $22 \pm 13$ & $5 \pm 4$ & $226 \pm 140$ \\
Linanthus dichotomus & $20 \pm 7$ & $.2 \pm .1$ & $8 \pm 3$ \\
Microseris linearis & $2 \pm 2$ & $.07 \pm .07$ & 35 \\
Phacelia fremontii & $2 \pm 2$ & $.02 \pm .02$ & 8 \\
P. vallis-mortae & $4 \pm 4$ & $.3 \pm .3$ & 160 \\
(Phlox stansburyi) & $36 \pm 15$ & $5.4 \pm 2.4$ & $160 \pm 48$ \\
Vulpia microstachys & $142 \pm 77$ & $1.4 \pm 0.8$ & $10 \pm 2$ \\
V. octoflora & $28 \pm 16$ & $.12 \pm .08$ & $3 \pm 1$ \\
Mean Quadrat Totals & $1166 \pm 229$ & $15 \pm 4$ & \\
& & & \\
\hline
\end{tabular}


Table 2.13 Ephemeral population characteristics on Plot YUF001, Yucca Flat baseline plot, replicate 1, measured April 14, 1988.

\begin{tabular}{lccc}
\hline Species & $\mathrm{n} / \mathrm{m}^{2}$ & $\mathrm{~g} / \mathrm{m}^{2}$ & $\mathrm{mg} /$ plant \\
\hline Astragalus lentiginosus & $2 \pm 2$ & $2 \pm 2$ & 1221 \\
Bromus rubens & $1872 \pm 556$ & $18 \pm 4$ & $14 \pm 1$ \\
B. tectorum & $20 \pm 11$ & $.5 \pm .3$ & $23 \pm 6$ \\
Chaenactis stevioides & $16 \pm 7$ & $.14 \pm .06$ & $9 \pm 2$ \\
Cryptantha pterocarya & $2 \pm 2$ & $.05 \pm .05$ & 24 \\
Descurainia pinnata & $2 \pm 2$ & $.008 \pm .008$ & 4 \\
Eriogonum nidularium & $10 \pm 10$ & $.04 \pm .04$ & 4 \\
Eriophyllum pringlei & $8 \pm 6$ & $.03 \pm .02$ & $4 \pm 0 \mathrm{y}$ \\
Gilia transmontana & $2 \pm 2$ & $.01 \pm .01$ & 5 \\
Lupinus flavoculatus & $2 \pm 2$ & $.09 \pm .09$ & $2 \pm 0$ \\
Malacothrix glabrata & $8 \pm 6$ & $.02 \pm .01$ & 1 \\
Phacelia fremontii & $2 \pm 2$ & $.002 \pm .002$ & \\
& $1956 \pm 557$ & $21 \pm 5$ & \\
Mean Quadrat Totals & & & \\
& & & \\
\hline
\end{tabular}

Table 2.14 Ephemeral population characteristics on Plot YUF001, replicate 2, measured on April 14, 1988.

\begin{tabular}{lccc}
\hline Species & $\mathrm{n} / \mathrm{m}^{2}$ & $\mathrm{~g} / \mathrm{m}^{2}$ & $\mathrm{mg} / \mathrm{plant}$ \\
\hline Bromus rubens & $1434 \pm 304$ & $22 \pm 4$ & $16 \pm 2$ \\
B. tectorum & $4 \pm 3$ & $.2 \pm .1$ & $38 \pm 23$ \\
Chaenactis carphoclinia & $2 \pm 2$ & $.1 \pm .1$ & 68 \\
C. stevioides & $8 \pm 4$ & $.2 \pm .2$ & $20 \pm 8$ \\
Cryptantha circumscissa & $2 \pm 2$ & $.004 \pm .004$ & 2 \\
C. recurvala & $4 \pm 4$ & $.02 \pm .02$ & 10 \\
Descurainia sophia & $2 \pm 2$ & $.01 \pm .01$ & 6 \\
Gilia transmontana & $2 \pm 2$ & $.01 \pm .01$ & $22 \pm 7$ \\
Lupinus flavoculatus & $8 \pm 5$ & $.2 \pm .1$ & $1 \pm 0$ \\
Machaeranthera canescens & $4 \pm 3$ & $.004 \pm .004$ & \\
Mean Quadrat Totals & $1470 \pm 306$ & $23 \pm 5$ & \\
& & & \\
\hline
\end{tabular}


Table 2.15 Ephemeral population characteristics on Plot YUF009, T1 ground zero, measured on April 26, 1988.

\begin{tabular}{lcrr}
\hline Species & $\mathrm{n} / \mathrm{m}^{2}$ & $\mathrm{~g} / \mathrm{m}^{2}$ & $\mathrm{mg} / \mathrm{plant}$ \\
\hline Bromus rubens & $1472 \pm 356$ & $14 \pm 4$ & $11 \pm 2$ \\
B. tectorum & $1884 \pm 396$ & $26 \pm 5$ & $17 \pm 4$ \\
B. unidentifiable & $268 \pm 142$ & $1.7 \pm 0.9$ & $7 \pm 2$ \\
Euphorbia albomarginata & $28 \pm 15$ & $.04 \pm .03$ & $6 \pm 2$ \\
Salsola sp. & $128 \pm 34$ & $.3 \pm .1$ & $2 \pm 0$ \\
Mean Quadrat Totals & $3880 \pm 340$ & $42 \pm 4$ & \\
& & & \\
\hline
\end{tabular}

Table 2.16 Ephemeral population characteristics on Plot YUF010, T1 ground zero control, measured on April 26, 1988.

\begin{tabular}{lrrr}
\hline Species & $\mathrm{n} / \mathrm{m}^{2}$ & $\mathrm{~g} / \mathrm{m}^{2}$ & $\mathrm{mg} /$ plant \\
\hline Bromus rubens & $188 \pm 49$ & $5 \pm 2$ & $42 \pm 21$ \\
B. tectorum & $2 \pm 2$ & $.01 \pm .01$ & 5 \\
Chaenactis stevioides & $4 \pm 3$ & $.03 \pm .02$ & $7 \pm 1$ \\
Chorizanthe thurberi & $6 \pm 3$ & $.2 \pm .1$ & $30 \pm 8$ \\
Cryptantha circumscissa & $12 \pm 6$ & $.11 \pm .08$ & $11 \pm 8$ \\
Eriogonum nidularium & $4 \pm 3$ & $.05 \pm .04$ & $12 \pm 8$ \\
Eriophyllum pringlei & $32 \pm 10$ & $.4 \pm .1$ & $15 \pm 4$ \\
Euphorbia albomarginata & $4 \pm 4$ & $.008 \pm .008$ & $2 \pm 2$ \\
Langloisia schottii & $4 \pm 3$ & $.01 \pm .01$ & 1 \\
Salsola sp. & $2 \pm 2$ & $.03 \pm .02$ & \\
Mean Quadrat Totals & $258 \pm 55$ & $6 \pm 2$ & \\
& & & \\
\hline
\end{tabular}


Table 2.17 Ephemeral population characteristics on Plot YUF011, 3B waste consolidation site, measured April 25, 1988.

\begin{tabular}{lccc}
\hline Species & $\mathrm{n} / \mathrm{m}^{2}$ & $\mathrm{~g} / \mathrm{m}^{2}$ & $\mathrm{mg} / \mathrm{plant}$ \\
\hline Salsola sp. & $12 \pm 5$ & $.7 \pm .4$ & $65 \pm 22$ \\
Mean Quadrat Totals & $12 \pm 5$ & $.7 \pm .4$ & \\
\hline
\end{tabular}

Table 2.18 Ephemeral population characteristics on Plot YUF012, 3B waste consolidation site control area, measured on April 25, 1988. (The unknown grass seedlings were probably perennials, and are excluded from the totals.)

\begin{tabular}{lrrc}
\hline Species & $\mathrm{n} / \mathrm{m}^{2}$ & $\mathrm{~g} / \mathrm{m}^{2}$ & $\mathrm{mg} / \mathrm{plant}$ \\
\hline Bromus rubens & $652 \pm 358$ & $15 \pm 2$ & $42 \pm 7$ \\
Cryptantha pterocarya & $2 \pm 2$ & $.2 \pm .2$ & 89 \\
C. recurvata & $12 \pm 7$ & $.2 \pm .1$ & $22 \pm 6$ \\
Descurainia pinnata & $2 \pm 2$ & $.04 \pm .04$ & 21 \\
D. sophia & $4 \pm 3$ & $.09 \pm .06$ & $54 \pm 50$ \\
Eriastrum eremicum & $4 \pm 3$ & $.2 \pm .1$ & $50 \pm 8$ \\
Eriophyllum pringlei & $2 \pm 2$ & $.03 \pm .03$ & 13 \\
Salsola sp. & $18 \pm 7$ & $.09 \pm .06$ & $4 \pm 1$ \\
(Unknown grass seedlings) & $24 \pm 24$ & $.03 \pm .03$ & 1 \\
& $696 \pm 334$ & $16 \pm 6$ & \\
Mean Quadrat Totals & & & \\
\hline
\end{tabular}


Table 2.19 Ephemeral population characteristics on Plot YUF014, T2-1 ground zero, measured May 9, 1988.

\begin{tabular}{lccr}
\hline Species & $\mathrm{n} / \mathrm{m}^{2}$ & $\mathrm{~g} / \mathrm{m}^{2}$ & $\mathrm{mg} / \mathrm{plant}$ \\
\hline & $774 \pm 169$ & $8 \pm 2$ & $13 \pm 2$ \\
Bromus rubens & $20 \pm 13$ & $.12 \pm .08$ & $5 \pm 2$ \\
Cryptantha circumscissa & $140 \pm 33$ & $1.7 \pm 0.4$ & $13 \pm 2$ \\
Erodium cicutarium & $44 \pm 14$ & $.10 \pm .04$ & $2 \pm 1$ \\
Salsola sp. & $978 \pm 151$ & $12 \pm 2$ & \\
Mean Quadrat Totals & & & \\
\hline
\end{tabular}

Table 2.20 Ephemeral population characteristics on Plot YUF015, T2-1 control area, measured May 9, 1988.

\begin{tabular}{lccc}
\hline Species & $\mathrm{n} / \mathrm{m}^{2}$ & $\mathrm{~g} / \mathrm{m}^{2}$ & $\mathrm{mg} / \mathrm{plant}$ \\
\hline & $658 \pm 344$ & $9 \pm 4$ & $22 \pm 5$ \\
Bromus rubens & $4 \pm 4$ & $.08 \pm .08$ & 20 \\
B. tectorum & $2 \pm 2$ & $.06 \pm .06$ & 29 \\
Chorizanthe rigida & $8 \pm 8$ & $.02 \pm .02$ & 3 \\
Cryptantha micrantha & $2 \pm 2$ & $.05 \pm .05$ & 27 \\
Eriogonum nidularium & $82 \pm 33$ & $5 \pm 2$ & $132 \pm 85$ \\
Erodium cicutarium & $2 \pm 2$ & $.1 \pm .1$ & 54 \\
Gilia transmontana & $2 \pm 2$ & $.004 \pm .004$ & 2 \\
Salsola sp. & $2 \pm 2$ & $.03 \pm .03$ & 17 \\
Sisymbrium altissimum & & $14 \pm 4$ & \\
Mean Quadrat Totals & $762 \pm 360$ & & \\
\hline
\end{tabular}


Table 2.21 Ephemeral population characteristics on Plot YUF016, Sedan, 305 meters from ground zero, measured on April 28, 1988.

\begin{tabular}{lccc}
\hline Species & $\mathrm{n} / \mathrm{m}^{2}$ & $\mathrm{~g} / \mathrm{m}^{2}$ & $\mathrm{mg} / \mathrm{plant}$ \\
\hline Astragalus lentiginosus & $3 \pm 3$ & $9 \pm 9$ & 3514 \\
Bromus rubens & $19 \pm 11$ & $.4 \pm .2$ & $23 \pm 8$ \\
B. tectorum & $1155 \pm 431$ & $29 \pm 9$ & $47 \pm 12$ \\
B. unidentifiable & $29 \pm 15$ & $.3 \pm .2$ & $26 \pm 20$ \\
Cryptantha circumscissa & $5 \pm 5$ & $.2 \pm .2$ & 28 \\
Descurainia sophia & $3 \pm 3$ & $.2 \pm .2$ & 86 \\
Eriogonum nidularium & $3 \pm 3$ & $.003 \pm .003$ & 1 \\
Gilia sinuata & $3 \pm 3$ & $.06 \pm .06$ & 24 \\
Mentzelia albicaulis & $3 \pm 3$ & $.3 \pm .3$ & 100 \\
Salsola sp. & $557 \pm 217$ & $2 \pm 1$ & $5 \pm 2$ \\
& $1779 \pm 525$ & $42 \pm 12$ & \\
Mean Quadrat Totals & & & \\
\hline
\end{tabular}

Table 2.22 Ephemeral population characteristics on Plot YUF017, Sedan, 914 meters from ground zero, measured on April 28, 1988.

\begin{tabular}{lccc}
\hline Species & $\mathrm{n} / \mathrm{m}^{2}$ & $\mathrm{~g} / \mathrm{m}^{2}$ & $\mathrm{mg} /$ plant \\
\hline Bromus rubens & $324 \pm 154$ & $4 \pm 2$ & $9 \pm 2$ \\
B. tectorum & $2004 \pm 424$ & $26 \pm 7$ & $19 \pm 6$ \\
B. unidentifiable & $36 \pm 24$ & $.5 \pm .3$ & $18 \pm 12$ \\
Chaenactis carphoclinia & $4 \pm 4$ & $.004 \pm .004$ & 1 \\
Cryptantha circumscissa & $28 \pm 12$ & $.11 \pm .05$ & $4 \pm 1$ \\
Erodium cicutarium & $8 \pm 8$ & $.5 \pm .5$ & 66 \\
Euphorbia albomarginata & $8 \pm 5$ & $.13 \pm .09$ & $16 \pm 4$ \\
Salsola sp. & $60 \pm 27$ & $.12 \pm .06$ & $2 \pm 0$ \\
Mean Quadrat Totals & $2469 \pm 493$ & $31 \pm 8$ & \\
\hline
\end{tabular}


Table 2.23 Ephemeral population characteristics on Plot YUF018, Sedan, 1524 meters from ground zero, measured May 2, 1988.

\begin{tabular}{lrrc}
\hline Species & $\mathrm{n} / \mathrm{m}^{2}$ & $\mathrm{~g} / \mathrm{m}^{2}$ & $\mathrm{mg} /$ plant \\
\hline & $142 \pm 69$ & $5 \pm 3$ & $33 \pm 13$ \\
Bromus rubens & $2 \pm 2$ & $.02 \pm .02$ & 10 \\
B. tectorum & $10 \pm 6$ & $.2 \pm .1$ & $17 \pm 2$ \\
Chaenactis stevioides & $2 \pm 2$ & $.002 \pm .002$ & 1 \\
Cryptantha circumscissa & $4 \pm 4$ & $.2 \pm .2$ & 36 \\
Descurainia pinnata & $4 \pm 3$ & $.10 \pm .07$ & $24 \pm 1$ \\
Gilia sinuata & $2 \pm 2$ & $.08 \pm .08$ & 40 \\
Ipomopsis polycladon & $10 \pm 5$ & $.04 \pm .02$ & $4 \pm 1$ \\
Lepidium lasiocarpum & $2 \pm 2$ & $.006 \pm .006$ & 3 \\
Oxytheca perfoliata & $2 \pm 2$ & $.05 \pm .05$ & 27 \\
Sisymbrium altissimum & $180 \pm 71$ & $5 \pm 3$ & \\
Mean Quadrat Totals & & & \\
\hline
\end{tabular}

Table 2.24 Ephemeral plant population characteristics on Plot PAM001, Pahute Mesa baseline plot, measured on May 23, 1988. (Phlox stansburyi, an herbaceous perennial, is excluded from the totals.)

\begin{tabular}{lrrr}
\hline Species & $\mathrm{n} / \mathrm{rn}^{2}$ & $\mathrm{~g} / \mathrm{m}^{2}$ & $\mathrm{mg} /$ plant \\
\hline Astragalus calycosus & $1.0 \pm 0.7$ & $.02 \pm .02$ & $23 \pm 5$ \\
Cryptantha pterocarya & $1.0 \pm 0.7$ & $.01 \pm .01$ & $10 \pm 6$ \\
Descurainia pinnata & $2 \pm 1$ & $.03 \pm .02$ & $14 \pm 5$ \\
Gilia transmontana & $28 \pm 12$ & $.5 \pm .2$ & $16 \pm 3$ \\
Phacelia fremontii & $.5 \pm .5$ & $.004 \pm .004$ & 9 \\
(Phlox stansburyi) & $(2 \pm 1)$ & $(.4 \pm .2)$ & $102 \pm 30$ \\
Mean Quadrat Totals & $32 \pm 12$ & $.5 \pm .2$ & \\
\hline
\end{tabular}


Table 2.25 Ephemeral and herbaceous perennial population characteristics on Plot RAM001, Rainier Mesa baseline plot, measured May 25, 1988. (Herbaceous perennials are excluded from the totals.)

\begin{tabular}{lccc}
\hline Species & $\mathrm{n} / \mathrm{m}^{2}$ & $\mathrm{~g} / \mathrm{m}^{2}$ & $\mathrm{mg} /$ plant \\
\hline (Chaenactis douglassii) & $(.5 \pm .5)$ & $(.01 \pm .01)$ & 21 \\
(Eriogonum umbellatum) & $(.5 \pm .5)$ & $(.1 \pm .1)$ & 194 \\
Gayophytum decipiens & $.5 \pm .5$ & $.004 \pm .004$ & 9 \\
(Poa sandbergii) & $(.5 \pm .5)$ & $(.07 \pm .07)$ & 140 \\
(Streptanthus cordatus) & $(.5 \pm .5)$ & $(.08 \pm .08)$ & 152 \\
Mean Quadrat Totals & $.5 \pm .5$ & $.004 \pm .004$ & \\
\hline
\end{tabular}

Table 2.26 Dead annuals on a site within a burned area in a valley within Yucca Mountain. The fire occurred June 21; dead grass was measured June 29, 1988.

\begin{tabular}{lcrc}
\hline Species & $\mathrm{n} / \mathrm{m}^{2}$ & $\mathrm{~g} / \mathrm{m}^{2}$ & $\mathrm{mg} / \mathrm{plant}$ \\
\hline Bromus rubens & $1460 \pm 380$ & $24 \pm 6$ & $14 \pm 2$ \\
B. tectorum & $700 \pm 140$ & $20 \pm 5$ & $26 \pm 5$ \\
B. unidentifiable & $60 \pm 60$ & $3 \pm 3$ & 49 \\
Mean Quadrat Totals & $2220 \pm 434$ & $47 \pm 13$ & \\
\hline
\end{tabular}


Table 2.27 Species presence-absence on plots sampled in 1988 for ephemeral plants. ( $\mathrm{A}=$ censused in quadrats (densities and biomasses are in Tables 2.1-2.26); $\mathrm{B}=$ present in $100 \mathrm{~m}^{2} ; \mathrm{C}=$ present in $1000 \mathrm{~m}^{2} ; \mathrm{D}=$ present only near the plot; - = not seen; ${ }^{*}=$ introduced species of ephemerals encountered in 1988).

\begin{tabular}{|c|c|c|c|c|c|c|c|}
\hline Location-> & FRF & FRF & FRF & FRF & JAF & MER & MER \\
\hline Plot $\rightarrow$ & 001 & 001 & 002 & 002 & 001 & 001 & 001 \\
\hline Replicate-> & $\mathbf{A}$ & B & & $\mathrm{C}$ & & $\mathrm{C}$ & \\
\hline \multicolumn{8}{|l|}{ Species } \\
\hline Allium nevadense & - & - & - & - & - & - & - \\
\hline Amsinckia tessellata & A & B & B & A & - & - & - \\
\hline Anisocoma acaulis & B & $\mathrm{C}$ & - & $\mathrm{C}$ & - & - & - \\
\hline Arabis pulchra & - & - & - & $\mathrm{C}$ & - & - & - \\
\hline Arenaria congesta & - & - & - & - & - & - & - \\
\hline Astragalus acutirostris & - & - & B & A & - & - & - \\
\hline A. calycosus calycosus & - & - & - & - & - & - & - \\
\hline A. didymocarpus & -. & - & - & - & A & - & - \\
\hline A. lentiginosus fremontii & - & - & - & - & $\ldots$ & - & - \\
\hline A. purshii tinctus & - & - & - & - & - & - & - \\
\hline A. tidestromii & - & - & - & - & - & - & C \\
\hline Baileya multiradiata & - & - & - & - & - & - & C \\
\hline Bromus rubens* & A & A & A & A & A & A & A \\
\hline B. tectorum ${ }^{*}$ & A & - & - & - & B & A & A \\
\hline B. trinii* & - & - & - & - & - & - & - \\
\hline B. unidentifiable* & - & - & - & - & - & - & - \\
\hline Calochortus flexuosus & - & - & - & - & - & - & - \\
\hline C. unidentified species & - & - & - & - & - & - & - \\
\hline Calycoseris wrightii & - & - & - & $\infty$ & - & - & - \\
\hline \multicolumn{8}{|l|}{ Camissonia boothii } \\
\hline ssp. condensata & - & - & - & - & - & - & - \\
\hline C. claviformis integrior & $B$ & - & - & - & - & C & $\mathrm{C}$ \\
\hline C. kernensis var. gilmanii & - & C. & B & - & - & - & - \\
\hline C. pterosperma & - & - & - & A & - & - & - \\
\hline Castilleja chromosa & - & - & - & - & - & - & - \\
\hline Caulanthus cooperi & - & - & - & - & - & $\mathrm{C}$ & $\mathrm{C}$ \\
\hline C. lasiophyllus & - & - & - & - & - & - & C \\
\hline Chaenactis carphoclinia & - & - & - & - & - & - & - \\
\hline C. douglassii & - & - & - & - & - & - & - \\
\hline C. fremontii & A & A & - & - & - & - & - \\
\hline
\end{tabular}


Table 2.27 Species presence-absence on plots sampled in 1988 for ephemeral plants. $(A=$ (Continued) censused in quadrats (densities and biomasses are Tables 2.1-2.26); $\mathrm{B}=$ present in $100 \mathrm{~m}^{2} ; \mathrm{C}=$ present in $1000 \mathrm{~m}^{2} ; \mathrm{D}=$ present only near the plot; - = not seen; ${ }^{*}=$ introduced species of ephemerals encountered in 1988).

Location $\rightarrow>$

Plot $\rightarrow$

Replicate->

Species
C. stevioides
C. xantiana

Chorizanthe brevicornu

C. rigida

C. thurberi

Crepis intermedia

Cryptantha angustifolia

C. circumscissa

C. dumetorum

C. flavoculata

C. gracilis

C. micrantha

C. nevadensis

C. pterocarya

C. recurvata

C. virginensis

Cuscuta spp.

Cymopteris ripleyi

Delphinium parishii

Descurainia pinnata

D. sophia*

Eriastrum eremicum

Eriogonum brachypodum

E. glandulosum

E. inflatum

E. maculatum

E. nidularium

E. ovalifolium ovalifolium

E. $s p$. (unidentifiable)

E. umbellatum subardum

Eriophyllum pringlei

$\begin{array}{ccccccc}\text { FRF } & \text { FRF } & \text { FRF } & \text { FRF } & \text { JAF } & \text { MER } & \text { MER } \\ 001 & 001 & 002 & 002 & 001 & 001 & 001 \\ \text { A } & \text { B } & & \text { C } & & \text { C } & \end{array}$

$$
\begin{aligned}
& \mathrm{C} \\
& - \\
& - \\
& \hline
\end{aligned}
$$

\begin{tabular}{l}
$\mathrm{C}$ \\
- \\
- \\
\hline
\end{tabular}

A

B

B

B

A

A 
Table 2.27 Species presence-absence on plots sampled in 1988 for ephemeral plants. $(A=$ (Continued) censused in quadrats (densities and biomasses are in Tables 2.1-2.26); B = present in $100 \mathrm{~m}^{2} ; \mathrm{C}=$ present in $1000 \mathrm{~m}^{2} ; \mathrm{D}=$ present only near the plot; - = not seen ${ }^{*}=$ introduced species of ephemerals encountered in 1988).

Location->

Plot $\rightarrow$

Replicate->

Species

Erodium cicutarium ${ }^{*}$

Escholzia glyptosperma

Euphorbia albomarginata

Fritellaria atropurpurea

Gayophytum decipiens

Gilia cana

G. sinuata

G. transmontana

Glyptopleura marginata

Halogeton glomeratus*

Ipomopsis congesta

1. polycladon

Langloisia schottii

L. setosissima

Lepidium lasiocarpum

Lesquerella kingii

Linanthus arenicola

L. demissus

L. dichotomius

L. jonesii

L. nuttallii

Lomatium nevadense

Lupinus flavoculat us

L. shockleyi

Machaeranthera canescens

Malacothrix glabrata

M. sonchoides

Mentzelia albicaulis

$M$. congesta

Microseris linearifolia

Monoptilon bellidiforme

$\begin{array}{ccccccc}\text { FRF } & \text { FRF } & \text { FRF } & \text { FRF } & \text { JAF } & \text { MER } & \text { MER } \\ 001 & 001 & 002 & 002 & 001 & 001 & 001 \\ \text { A } & \text { B } & & \text { C } & & \text { C } & \end{array}$

$\begin{array}{lllllll}\text { C } & - & \text { A } & - & - & \text { A } & \text { A } \\ \text { B } & \text { B } & \text { B } & - & - & - & - \\ \text { C } & - & \text { C } & - & - & - & - \\ - & - & - & - & - & - & - \\ - & - & - & - & - & - & - \\ - & - & \text { C } & - & - & - & - \\ \text { A } & \text { A } & - & \text { A } & - & - & - \\ - & - & - & \text { B } & - & \text { A } & \text { A } \\ - & - & - & - & - & - & - \\ - & - & - & - & - & - & \text { C } \\ - & - & - & - & - & - & - \\ - & - & - & - & - & \text { A } & \text { A } \\ - & - & - & - & - & - & - \\ - & - & - & \text { A } & - & \text { A } & \text { A } \\ - & - & - & \text { A } & - & - & \text { A } \\ - & - & - & - & - & - & - \\ - & - & \text { A } & \text { A } & - & - & - \\ - & - & - & - & - & - & - \\ - & - & - & - & - & - & - \\ - & - & - & - & - & - & - \\ - & - & - & - & - & - & - \\ - & - & - & - & - & - & - \\ - & - & - & - & - & - & - \\ - & - & - & \text { B } & - & - & - \\ \text { C } & \text { C } & - & - & - & \text { C } & \text { D } \\ - & \text { C } & - & - & - & \text { C } & - \\ - & - & - & - & - & - & - \\ - & - & - & - & \text { A } & \text { B } & \text { B } \\ - & - & - & - & - & - & - \\ - & - & - & - & - & - & - \\ - & - & - & - & - & - & - \\ - & - & - & - & -\end{array}$


Table 2.27 Species presence-absence on plots sampled in 1988 for ephemeral plants. $A=$ (Continued) censused in quadrats (densities and biomasses are in Tables 2.1-2.26); B = present in $100 \mathrm{~m}^{2} ; \mathrm{C}=$ present in $1000 \mathrm{~m}^{2} ; \mathrm{D}=$ present only near the plot; - = not seeni).

Location->

Plot $\rightarrow$

Replicate->

Species

Nama demissum

N. densum densum

Nemacladus glanduliferus

var. orientalis

Oenothera caespitosa

O. primiveris

Oxytheca perfoliata

Pectocarya heterocarpa

$P$. platycarpa

P. setosa

Penstemon sp.

Phacelia crenulata

$P$. fremontii

P. vallis-mortae

Phlox stansburyi

Pou sandbergii

Rafinesquia neomexicana

Salsole spp.

Schismus sp.

Sisymbrium altissimum

Streptanthella longirostris

Stre,stanthus cordatus

Stylocline micropoides

Syntrichopappus fremontii

Viguiera multiflore var. nevadensis

Vulpia microstachys pruciflora

V. actoflora

$\begin{array}{ccccccc}\text { FRF } & \text { FRF } & \text { FRF } & \text { FRF } & \text { JAF } & \text { MER } & \text { MER } \\ 001 & 001 & 002 & 002 & 001 & 001 & 001 \\ \text { A } & \text { B } & & \text { C } & & \text { C } & \end{array}$

C

A

C - - A

-

c - - -

$-\quad-\mathrm{C}-{ }^{-}-\mathrm{B}$

- B - - -7 -

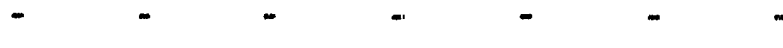

- $\quad-\quad-\quad-$

A A A A A A

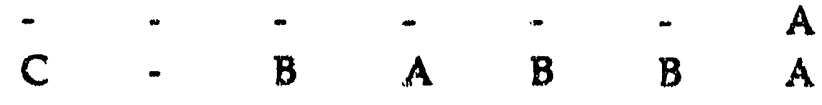

- $\quad-\quad-\quad-\quad-\quad-\quad-$ 
Table 2.28 Species presence-absence on plots sampled in 1988 for ephemeral plants. $(A=$ censused in quadrats (densities and biomasses are in Tables 2.1-2.26); $\mathrm{B}=$ present in $100 \mathrm{~m}^{2} ; \mathrm{C}=$ present in $100 \mathrm{~m} \mathrm{~m}^{2} ; \mathrm{D}=$ present only near the plot; - = not seen).

Location->

Plot $\rightarrow>$

Replicate->

Species

Allium nevadense

Amsinckia tessellata

Anisocoma acaulis

Arabis pulchra

Arenaria congesta

Astragalus acutirostris

A. calycosus calycosus

A. didymocarpus

A. lentiginosus fremontii

A. purshii tinctus

A. tidestromii

Baileya multiradiata

Bromus rubens

B. tectorum

B. trinii

B. unidentifiable

Calochortus flexuosus

C. unidentified species

Calycoseris wrightii

Camissonia boothii

ssp. condensata

C. claviformis integrior

C. kernensis var. gilmamii

C. pterosperma

Castilleja chromosa

Caulanthus cooperi

C. lasiophyllus

Chaenact is carphoclinia

C. douglassii

C. fremontii

C. stevioides

$\begin{array}{lllcccc}\text { MER } & \text { ROV } & \text { ROV } & \text { YUF } & \text { YUF } & \text { YUF } & \text { YUF } \\ 002 & 005 & 006 & 001 & 001 & 011 & 012 \\ & & & \text { A } & \text { B } & & \end{array}$


Table 2.28 Species presence-absence on plots sampled in 1988 for ephemeral plants." ( $\mathrm{A}=$ (Continued) censused in quadrats (densities and biomasses are in Tables 2.1-2.26); $\mathrm{B}=$ present in $100 \mathrm{~m}^{2} ; \mathrm{C}=$ present in $1000 \mathrm{~m}^{2} ; \mathrm{D}=$ present only near the plot; - = not seen).

Location->

Plot $\rightarrow$

Replicatem

Species

\section{C. xantiana}

Chorizanthe brevicomu

C. rigida

C. thurberi

Crepis intermedia

Cryptantha angustifolia

C. circumscissa

C. dumetorum

C. flavoculata

C. gracilis

C. micrantha

C. nevadensis

C. plerocarya

C. recurvata

C. virginensis

Cuscuta spp.

Cymopteris ripleyi

Delphinium parishii

Descurainia pinnata

D. sophia

Eriastrum eremicum

Eriogonum brachypodum

E. glandulosum

E. inflatum

E. maculatum

E. nidularium

E. ovalifolium ovalifolium

E. sp. (unidentifiable)

E. umbellatum subardum

Eriophyllum pringlei

Eradium cicutarium
MER ROV ROV YUF YUF YUF YUF

$\begin{array}{lllllll}002 & 005 & 006 & 001 & 001 & 011 & 012\end{array}$

A B
B

D

A

A

D

A

A

A

D

C

B

A

A

$\begin{array}{llllll}\text { A } & \text { A } & \text { A } & \text { C } & - & \text { A } \\ \text { C } & \text { C } & - & - & - & -\end{array}$ 
Table 2.28 Species presence-absence on plots sampled in 1988 for ephemeral plants. ( $A=$ (Continued) censused in quadrats (densities and biomasses are in Tables 2.1-2.26); $B=$ present in $100 \mathrm{~m}^{2} ; \mathrm{C}=$ present in $1000 \mathrm{~m}^{2} ; \mathrm{D}=$ present only near the plot; $-=$ not seen).

$\begin{array}{lllllllll}\text { Location }> & \text { MER } & \text { ROV } & \text { ROV } & \text { YUF } & \text { YUF } & \text { YUF } & \text { YUF } \\ \text { Plot }-> & 002 & 005 & 006 & 001 & 001 & 011 & 012 \\ \text { Replicate } \rightarrow & & & & & \text { A } & \text { B } & & \end{array}$

Escholzia glyptosperma

Euphorbia albomarginata

Fritellaria atropurpurea

Gayophytum decipiens

Gilia cana

G. sinuata

G. transmontana

Glyptopleura marginata

Halogeton glomeratus

Ipriniupsis congesta

I. polycladon

Inngloisia schottii

L. setosiisima

Lepidium lasiocarpum

Lesquerella kingii

Linanthus arenicola

L. demissus

L. dichotomus

L. jonesii

L. nuttallii

Lomatium nevadense

Lupinus flavoculatus

L. shockleyi

Machaeranthera canescens

Malacothrix glabrata

$M$. sonchoides

Mentzelia albicaulis

$M$. congesta

Microseris linearifolia

\begin{tabular}{|c|c|c|c|c|c|}
\hline - & B & - & - & - & - \\
\hline A & - & .* & B & - & - \\
\hline - & - & - & - & - & - \\
\hline - & - & - & - & - & - \\
\hline - & - & - & - & - & - \\
\hline - & - & - & - & - & - \\
\hline A & B & $A$ & A & A & - \\
\hline - & - & - & - & - & - \\
\hline - & - & - & - & - & - \\
\hline - & - & - & - & - & - \\
\hline - & B & - & $C$ & B & - \\
\hline- & - & B & B & - & - \\
\hline- & "- & " & - & - & - \\
\hline- & C & C & B & - & - \\
\hline - & - & - & - & - & - \\
\hline - & - & - & - & - & - \\
\hline- & $A$ & - & - & - & - \\
\hline - & - & - & - & ". & - \\
\hline - & - & $"$ & - & - & - \\
\hline- & - & - & - & - & - \\
\hline- & - & - & - & - & - \\
\hline - & - & - & A & A & - \\
\hline - & - & B & - & - & - \\
\hline B & - & - & B & A & - \\
\hline - & $C$ & A & A & - & - \\
\hline - & - & - & - & - & - \\
\hline - & - & B & - & - & - \\
\hline - & - & - & - & - & ". \\
\hline- & - & $\sim$ & - & - & $=$ \\
\hline
\end{tabular}


Table 2.28 Species presence-absence on plots sampled in 1988 for ephemeral plants. $(A=$ (Continued) censused in quadrats (densities and biomasses are in Tables 2.1-2.26); $\mathrm{B}=$ present in $100 \mathrm{~m}^{2} ; \mathrm{C}=$ present in $1000 \mathrm{~m}^{2} ; \mathrm{D}=$ present only near the plot; - = not seen).

Location->

Plot $\rightarrow$

Replicate->

Species

Monoptilon bellidiforme

Nama demissum

N. densum densum

Nemacladus glanduliferus var. orientalis

Oenothera caespitosa

O. primiveris

Oxytheca perfoliata

Pectocarya heterocarpa

$P$. platycarpa

$P$. setosa

Penstemon sp.

Phacelia crenulata

$P$. fremontii

$P$. vallis-mortae

Phlox stansburyi

Poa sardbergii

Rafinesquia neomexicana

Salsola spp.

Schismus sp.

Sisymbrium altissimum

Streptanthella longirostris

Streptanthus cordatus

Stylocline micropoides

Syntrichopappus fremontii

Viguiera multiflora var. nevadensis

Vulpia microstachys pauciflora

$V$. octoflora

$\begin{array}{lllcccc}\text { MER } & \text { ROV } & \text { ROV } & \text { YUF } & \text { YUF } & \text { YUF } & \text { YUF } \\ 002 & 005 & 006 & 001 & 001 & 011 & 012 \\ & & & \text { A } & \text { B } & & \end{array}$

\begin{tabular}{|c|c|c|c|c|c|c|}
\hline - & $C$ & $\mathbf{A}$ & - & - & - & - \\
\hline - & - & A & - & - & - & - \\
\hline - & - & - & - & - & - & - \\
\hline - & $C$ & - & - & - & - & - \\
\hline .. & - & - & - & - & - & - \\
\hline - & - & - & - & - & - & - \\
\hline- & $C$ & - & - & - & - & B \\
\hline - & B & - & - & - & - & - \\
\hline- & B & - & - & - & - & - \\
\hline - & - & - & - & - & - & - \\
\hline - & - & - & - & - & - & - \\
\hline - & $C$ & - & - & - & - & - \\
\hline - & $\mathbf{A}$ & - & A & - & - & C \\
\hline - & B & B & C & - & - & - \\
\hline - & - & - & - & - & - & - \\
\hline - & - & - & - & - & - & . \\
\hline - & - & $A$ & - & - & - & - \\
\hline - & - & - & - & - & A & $A$ \\
\hline - & - & - & - & - & - & - \\
\hline- & - & - & - & - & - & $B$ \\
\hline - & $\mathbf{A}$ & - & - & - & - & B \\
\hline 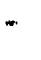 & - & - & - & - & - & - \\
\hline - & B & - & - & - & - & - \\
\hline - & $\cdot$ & - & - & - & - & \\
\hline - & - & - & - & - & - & - \\
\hline - & - & - & - & - & - & - \\
\hline$A$ & A & A & - & - & - & - \\
\hline
\end{tabular}


Table 2.29 Species presence-absence on plots sampled in 1988 for ephemeral plants. $(\mathrm{A}=$ censused in quadrats (densities and biomasses are in Tables 2.1-2.26); $\mathrm{B}=$ present in $100 \mathrm{~m}^{2} ; \mathrm{C}=$ present in $1000 \mathrm{~m}^{2} ; \mathrm{D}=$ present only near the plot; $-=$ not seen).

Location->

Plot $\rightarrow$

Replicate->

Species

Allium nevadense

Amsinckia tessellata

Anisocoma acaulis

Arabis pulchra

Arenaria congesta

Astragalus acutirostris

A. calycosus calycosus

A. didymocarpus

A. lentiginosus fremontii

A. purshii tinctus

A. tidestromii

Baileya multiradiata

Bromus rubens

B. tectorum

B. trinii

B. unidentifiable

Calochortus flexuosus

C. unidentified species

Calycoseris wrightii

Camissonia boothii

ssp. condensa

C. claviformis integrior

C. kernensis var. gilmanii

C. pterosperma

Castilleja chromosa

Caulanthus cooperi

C. lasiophyllus

Chaenactis carphoclinia

C. douglassii

C. fremontii

C. stevioides

$\begin{array}{lllllll}\text { YUF } & \text { YUF } & \text { YUF } & \text { YUF } & \text { YUF } & \text { YUF } & \text { YUF } \\ 009 & 010 & 014 & 015 & 016 & 017 & 018\end{array}$

$\begin{array}{lllllll}- & - & - & - & - & - & - \\ \text { C } & \text { B } & - & - & \text { C } & \text { B } & \text { C } \\ - & - & - & - & - & - & - \\ - & \text { C } & - & - & - & - & - \\ - & - & - & - & - & - & - \\ - & - & - & - & - & - & - \\ - & - & - & - & - & - & - \\ - & - & - & - & - & - & - \\ \text { B } & \text { B } & - & \text { B } & \text { A } & \text { C } & - \\ - & - & - & - & - & - & - \\ - & - & - & - & - & - & - \\ - & - & - & - & - & - & -\end{array}$

$\begin{array}{lllllll}\text { A } & \text { A } & \text { A } & \text { A } & \text { A } & \text { A } & \text { A }\end{array}$

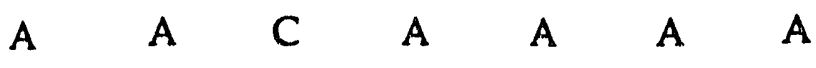

A

-

A

A

A -

$-$

B

- - -$$
\begin{array}{lllllll}
- & - & - & \text { C } & - & - & - \\
- & - & - & - & - & - & - \\
- & - & - & - & - & - & \text { B } \\
- & - & - & - & - & - & - \\
- & - & - & - & - & - & - \\
- & - & - & - & - & - & - \\
- & - & - & - & - & - & - \\
- & - & - & - & - & \text { A } & \text { C } \\
- & - & - & - & - & - & - \\
- & - & - & \text { B } & - & - & - \\
\text { C } & \text { A } & - & - & - & - & -
\end{array}
$$ 
Table 2.29 Species presence-absence on plots sampled in 1988 for ephemeral plants. $(A=$ (Continued) censused in quadrats (densities and biomasses are in Tables 2.1-2.26); $\mathrm{B}=$ present in $100 \mathrm{~m}^{2} ; \mathrm{C}=$ present in $1000 \mathrm{~m}^{2} ; \mathrm{D}=$ present only near the plot; - = not seen).

Location $\rightarrow>$

Plot ->

Replicate->

Species

C. xantiana

Chorizanthe brevicormu

C. rigida

C. thurberi

Crepis intermedia

Cryptantha angustifolia

C. circumscissa

C. dumetorum

C. flavoculata

C. gracilis

C. micrantha

C. nevadensis

C. pterocarya

C. recurvata

C. virginensis

Cuscuta spp.

Cymopteris ripleyi

Delphinium parishii

Descurainia pinnata

D. sophia

Eriastrum eremicum

Eriogonum brachypodum

E. glandulosum

E. inflatum

E. maculatum

E. nidularium

E. ovalifolium ovalifolium

E. sp. (unidentifiable)

E. umbellatum subardum

Eriophyllum pringlei

Erodium cicutarium
YUF YUF YUF YUF YUF YUF YUF

$\begin{array}{lllllll}009 & 010 & 014 & 015 & 016 & 017 & 018\end{array}$


Table 2.29 Species presence-absence on plots sampled in 1988 for ephemeral plants. ( $\mathrm{A}=$ (Continued) censused in quadrats (densities and biomasses are in Tables 2.1-2.26); $\mathrm{B}=$ present in $100 \mathrm{~m}^{2} ; \mathrm{C}=$ present in $1000 \mathrm{~m}^{2} ; \mathrm{D}=$ present only near the plot; .$=$ not seen).

\begin{tabular}{|c|c|c|c|c|c|c|c|}
\hline Location-> & YUF & YUF & YUF & YUF & YUF & YUF & YUF \\
\hline $\begin{array}{l}\text { Plot }-> \\
\text { Replicate-> } \\
\text { Species }\end{array}$ & 009 & 010 & 014 & 015 & 016 & 017 & 018 \\
\hline Escholzia glyptosperma & - & - & - & - & - & - & - \\
\hline Euphorbia albomarginata & A & A & $\mathrm{C}$ & - & - & A & - \\
\hline Fritellaria atropurpurea & - & - & - & - & - & - & - \\
\hline Gayophytum decipiens & - & - & - & - & - & - & - \\
\hline Gilia cana & - & - & - & - & - & - & - \\
\hline G. sinuata & - & - & - & - & A & A & $A$ \\
\hline G. transmontana & - & B & - & A & B & - & B \\
\hline Glyptopleura marginata & - & - & - & - & - & - & C \\
\hline Halogeton glomeratus & - & - & - & - & - & - & - \\
\hline Ipomopsis congesta & - & - & - & - & - & - & - \\
\hline I. polycladon & - & - & - & $\mathrm{C}$ & - & - & A \\
\hline Langloisia schottii & - & A & - & - & - & - & - \\
\hline L. setosissima & - & - & - & - & - & - & - \\
\hline Lepidium lasiocarpum & - & - & - & - & - & - & A \\
\hline Lesquerella kingii & - & - & - & - & - & - & - \\
\hline Linanthus arenicola & - & - & - & - & - & - & - \\
\hline L. demissus & - & - & - & - & - & - & - \\
\hline L. dichotomus & 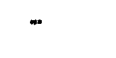 & - & - & - & - & - & - \\
\hline L. jonesii & - & - & - & - & - & - & - \\
\hline L. nuttallii & - & - & - & - & - & - & - \\
\hline Lomatium nevadense & - & - & - & - & - & - & - \\
\hline Lupinus flavoculatus & - & - & - & - & - & - & - \\
\hline L. shockleyi & - & - & - & B & - & - & - \\
\hline Machaeranthera canescens & C & $\mathrm{C}$ & - & - & - & $\mathrm{C}$ & $\mathrm{D}$ \\
\hline Malacothrix glabrata & - & C & $*$ & - & - & $\mathrm{C}$ & - \\
\hline M. sonchoides & - & - & - & - & - & - & - \\
\hline Mentzelia albicaulis & - & - & - & - & A & B & B \\
\hline M. congesta & - & - & - & - & - & - & - \\
\hline Microseris linearifolia & - & - & - & - & - & - & - \\
\hline
\end{tabular}


Table 2.29 Species presence-absence on plots sampled in 1988 for ephemeral plants. ( $A=$ (Continued) censused in quadrats (densities and biomasses are in Tables 2.1-2.26); $B=$ present in $100 \mathrm{~m}^{2} ; \mathrm{C}=$ present in $1000 \mathrm{~m}^{2} ; \mathrm{D}=$ present only near the plot; - = not seen).

Location->

Plot ->

Replicate->

Species

Monoptilon bellidiforme

Nama demissum

$N$. densum densum

Nemacladus glanduliferus var. orientalis

Oenothera caespitosa

0 . primiveris

Oxytheca perfoliata

Pectocarya heterocarpa

$P$. platycarpa

$P$. setosa

Penstemon sp.

Phacelia crenulata

P. fremontii

$P$. vallis-mortae

Phlox stansburyi

Poa sandbergii

Rafinesquia neomexicana

Salsola spp.

Schismus sp.

Sisymbrium altissimum

Streptanthella longirostris

Streptanthus cordatus

Stylocline micropoides

Syntrichopappus fremontii

Viguiera multiflora var.

nevadensis

Vulpia microstachys

pauciflora

V. octoflora

$\begin{array}{lllllll}\text { YUF } & \text { YUF } & \text { YUF } & \text { YUF } & \text { YUF } & \text { YUF } & \text { YUF } \\ 009 & 010 & 014 & 015 & 016 & 017 & 018\end{array}$

C

A

C - - B

$\begin{array}{llllll}- & \mathrm{B} & - & - & - & -\end{array}$

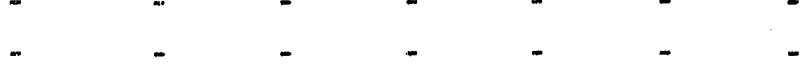

$\begin{array}{lllllll}- & - & - & - & - & - & - \\ \text { A } & \text { A } & \text { A } & \text { A } & \text { A } & \text { A } & -\end{array}$

$\begin{array}{llllll}A & A & A & A & A & A \\ - & - & - & - & - & -\end{array}$

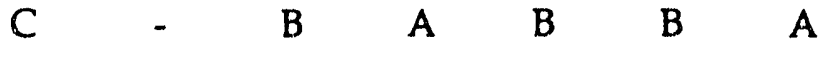

$\begin{array}{lllllllll}C & - & - & - & - & - & - & - & -\end{array}$

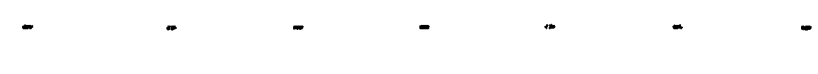

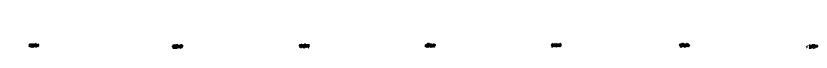


Table 2.30 Species presence-absence on plots sampled in 1988 for ephemeral plants. $(A=$ censused in quadrats (densities and biomasses are in Tables 2.1-2.26); B = present in $100 \mathrm{~m}^{2} ; \mathrm{C}=$ present in $1000 \mathrm{~m}^{2} ; \mathrm{D}=$ present only near the plot; - = not seen).

Location->

Plot $\rightarrow$

Replicate->

Species

Allium nevadense

Amsinckia tessellata

Anisocoma acaulis

Arabis pulchra

Arenaria congesta

Astragalus acutirostris

A. calycosus calycosus

A. didymocarpus

A. lentiginosus fremontii

A. purshii tinctus

A. tidestromii

Baileya multiradiata

Bromus rubens

B. tectorum

B. trinii

B. unidentifiable

Calochortus flexuosus

C. unidentified species

Calycoseris wrightii

Camissonia boothii

ssp. condensa

C. claviformis integrior

C. kernensis var.

C. pterosperma.

Castilleja chromosa

Caulanthus cooperi

C. lasiophyllus

Chaenactis carphoclinia

C. douglassii

C. fremontii

C. stevioides $\begin{array}{llll}\text { MID } & \text { MID } & \text { PAM } & \text { RAM } \\ 001 & 001 & 001 & 001\end{array}$

C

$\begin{array}{llll}- & \text { A } & - & - \\ - & \text { B } & - & - \\ - & - & - & - \\ - & \text { C } & \text { C } & - \\ - & - & - & \text { B } \\ - & \text { B } & - & - \\ - & - & \text { A } & - \\ - & - & - & - \\ \text { C } & \text { B } & \text { C } & - \\ \text { A } & \text { B } & - & - \\ - & - & \text { C } & - \\ - & - & - & -\end{array}$

A A C -

A A B -

A

- $\quad-$

$\begin{array}{llll}- & - & - & - \\ - & - & - & -\end{array}$

- $\quad-12$ 
Table 2.30 Species presence-absence on plots sampled in 1988 for ephemeral plants. $(A=$ (Continued) censused in quadrats (densities and biomasses are in Tables 2.1-2.26); $\mathrm{B}=$ present in $100 \mathrm{~m}^{2} ; \mathrm{C}=$ present in $1000 \mathrm{~m}^{2} ; \mathrm{D}=$ present only near the plot; - = not seen).

\section{Location $\rightarrow>$}

Plot $\rightarrow$

Replicate->

Species

C. xantiana

Chorizanthe brevicornu

C. rigida

C. thurberi

Crepis intermedia

Cryptantha angustifolia

C. circumscissa

C. dumetorum

C. flavoculata

C. gracilis

C. micrantha

C. nevadensis

C. pterocarya

C. recurvata

C. virginensis

Cuscuta spp.

Cymopteris ripleyi

Delphinium parishii

Descurainia pinnata

D. sophia

Eriastrum eremicum

Eriogonum brachypodum

E. glandulosum

E. inflatum

E. maculatum

E. nidularium

E. ovalifolium ovalifolium

E. sp. (unidentifiable)

E. umbellatum subardum

Eriophyllum pringlei

Erodium cicutarium

$\begin{array}{clll}\text { MID } & \text { MID } & \text { PAM } & \text { RAM } \\ 001 & 001 & 001 & 001 \\ \text { C } & & & \end{array}$

C

$\begin{array}{llll}- & - & - & - \\ B & - & - & - \\ - & - & - & - \\ A & - & - & - \\ - & - & - & \text { C } \\ - & - & - & - \\ A & - & - & - \\ - & - & - & - \\ - & - & \text { C } & \text { B } \\ - & - & - & - \\ - & \text { A } & - & - \\ - & - & - & - \\ \text { A } & \text { B } & \text { A } & - \\ - & - & - & - \\ - & - & - & - \\ - & - & - & - \\ - & - & - & - \\ - & - & - & -\end{array}$

A A A -

- $\quad-\quad C$ -

A A C -

A - - -

A $\quad-\quad-\quad-$

- -4

- $\quad-\quad$ -

$\begin{array}{llll}- & - & - & - \\ - & - & - & -\end{array}$

$\begin{array}{lll}- & - & -\end{array}$

- $\quad-\quad-$

- $\quad-\quad-\quad A$

$\begin{array}{llll}- & B & - & - \\ - & - & - & -\end{array}$ 
Table 2.30 Species presence-absence on plots sampled in 1988 for ephemeral plants. $(A=$ (Continued) censused in quadrats (densities and biomasses are in Tables 2.1-2.26); $\mathrm{B}=$ present in $100 \mathrm{~m}^{2} ; C=$ present in $1000 \mathrm{~m}^{2} ; \mathrm{D}=$ present only near the plot; $-=$ not seen).

$\begin{array}{lllll}\text { Location-> } & \text { MID } & \text { MID } & \text { PAM } & \text { RAM } \\ \text { Plot -> } & 001 & 001 & 001 & 001 \\ \text { Replicate-> } & \text { C } & & & \\ \text { Species } & & & & \\ \text { Escholzia glyptosperma } & - & - & - & - \\ \text { Euphorbia albomarginata } & \text { A } & - & - & - \\ \text { Fritellaria atropurpurea } & - & - & - & \text { B } \\ \text { Gayophytum decipiens } & - & - & - & \text { A } \\ \text { Gilia cana } & - & - & - & - \\ \text { G. sinuata } & \text { A } & \text { A } & - & - \\ \text { G. transmontana } & \text { A } & \text { A } & \text { A } & - \\ \text { Glyptopleura marginata } & - & - & - & - \\ \text { Halogeton glomeratus } & - & - & - & - \\ \text { Ipomopsis congesia } & - & - & \text { D } & \text { C } \\ \text { I. polycladon } & - & - & \text { C } & - \\ \text { Langloisia schottii } & - & - & - & - \\ \text { L. setosissima } & - & - & - & - \\ \text { Lepidium lasiocarpum } & - & - & - & - \\ \text { Lesquerella kingii } & - & - & - & \text { B } \\ \text { Linanthus arenicola } & - & - & - & - \\ \text { L. demissus } & - & - & - & - \\ \text { L. dichotomus } & \text { A } & - & - & - \\ \text { L. jonesii } & - & - & - & - \\ \text { L. nuttallii } & - & - & - & \text { B } \\ \text { Lomatium nevadense } & - & \text { C } & - & - \\ \text { Lupinus flavoculatus } & \text { C } & - & - & - \\ \text { L. shockleyi } & - & - & - & - \\ \text { Machaeranthera canescens } & - & \text { B } & - & - \\ \text { Malacothrix glabrata } & - & - & - & - \\ \text { M. sonchoides } & - & - & - & - \\ \text { Mentzelia albicaulis } & - & - & - & - \\ \text { M. congesta } & - & - & - & \text { B } \\ \text { Microseris linearifolia } & - & - & -\end{array}$


Table 2.30 Species presence-absence on plots sampled in 1988 for ephemeral plants. ( $A=$ (Continued) censused in quadrats (densities and biomasses are in Tables 2.1-2.26); $\mathrm{B}=$ present in $100 \mathrm{~m}^{2} ; \mathrm{C}=$ present in $1000 \mathrm{~m}^{2} ; \mathrm{D}=$ present only near the plot; - = not seen).

Location->

Plot $\rightarrow$

Replicate->

Species

Monoptilon bellidiforme

Nama demissum

$N$. densum densum

Nemacladus glanduliferus var. orientalis

Oenothera caespitosa

O. primiveris

Oxytheca perfoliata

Pectocarya heterocarpa

$P$. platycarpa

P. setosa

Penstemon sp.

Phacelia crenulata

P. fremontii

$P$. vallis-mortae

Phlox stansburyi

Poa sandbergii

Rafinesquia neomexicana

Salsola spp.

Schismus sp.

Sisymbrium altissimum

Streptanthella longirostris

Streptanthus cordatus

Stylocline micropoides

Syntrichopappus fremontii

Viguiera multiflora var.

nevadensis

Vulpia microstachys pauciflora

V. octoflora

$\begin{array}{clll}\text { MID } & \text { MID } & \text { PAM } & \text { RAM } \\ 001 & 001 & 001 & 001 \\ \text { C } & & & \end{array}$

C

$\begin{array}{llll}- & - & - & - \\ - & - & - & - \\ - & - & D & -\end{array}$

$-$

A - A -

A A - C

- $\quad-\quad-\quad A$

- $-\quad-\quad-$

A - -

$\begin{array}{llll}- & - & - & - \\ - & - & -\end{array}$

- -4

- - - -

- $\quad-\quad-\quad A$

C $\quad$ B -

$-2$

- B - -

$\begin{array}{llll}A & - & - & - \\ A & - & - & -\end{array}$ 
Table 2.31 Estimated soil water (0-30 cm depth; total $\mathrm{mm}$ of water 1-15) and precipitation $(\mathrm{mm})$ in Mercury, Nevada between July 1, 1987, and June 30, 1988.

\begin{tabular}{|c|c|c|c|c|c|}
\hline Date & Rain & $\begin{array}{c}\text { Soil } \\
\text { Water }\end{array}$ & Date & Rain & $\begin{array}{l}\text { Soil } \\
\text { Water }\end{array}$ \\
\hline 1 ЛU 87 & & -1.5 & 15 JAN 88 & 6.4 & +12.1 \\
\hline $10 \mathrm{JUL}$ & & -0.4 & $23 \mathrm{JAN}$ & 31.2 & +27.1 \\
\hline 17 JUL & 1.8 & -0.4 & $29 \mathrm{JAN}$ & & +16.9 \\
\hline $24 \pi \pi$ & 10.2 & +2.7 & 4 FEB & 0.8 & +12.7 \\
\hline 31 JUL & & +0.6 & 12 FEB & & +9.2 \\
\hline 11 AUG & & -0.5 & 19 FEB & & +6.6 \\
\hline 20 AUG & & -0.7 & $26 \mathrm{FEB}$ & & +0.7 \\
\hline $28 \mathrm{AUG}$ & & -0.7 & $4 \mathrm{MAR}$ & 6.8 & +8.0 \\
\hline 4 SEP & & -0.5 & $11 \mathrm{MAR}$ & & +8.6 \\
\hline 21 SEP & & -0.5 & $18 \mathrm{MAR}$ & & +6.3 \\
\hline 25 SEP & & -0.4 & $25 \mathrm{MAR}$ & & +4.7 \\
\hline 2 OCT & & -0.5 & $1 \mathrm{APR}$ & & +4.6 \\
\hline 9 OCT & & +0.1 & 8 APR & & +3.4 \\
\hline $16 \mathrm{OCT}$ & 6.9 & +10.6 & $18 \mathrm{APR}$ & 34.3 & +14.2 \\
\hline 23 OCT & 6.4 & +7.4 & 22 APR & 2.8 & +11.5 \\
\hline $04 \mathrm{NOV}$ & 25.4 & +13.8 & 29 APR & & +7.6 \\
\hline $06 \mathrm{NOV}$ & 15.5 & +15.2 & $6 \mathrm{MAY}$ & 2.0 & +7.5 \\
\hline $13 \mathrm{NOV}$ & & +14.0 & $13 \mathrm{MAY}$ & 2.0 & +3.6 \\
\hline $20 \mathrm{NOV}$ & 1.0 & +11.6 & $20 \mathrm{MAY}$ & & +1.3 \\
\hline $4 \mathrm{DEC}$ & & +11.8 & $27 \mathrm{MAY}$ & & +1.0 \\
\hline 17 DEC & 10.7 & +17.6 & $8 \pi \mathrm{UN}$ & 2.5 & +0.1 \\
\hline $23 \mathrm{DEC}$ & 7.1 & +19.3 & 17 JUN & & -0.2 \\
\hline 7 JAN 88 & 6.4 & +15.8 & $24 \pi U N$ & 3.0 & -0.5 \\
\hline
\end{tabular}


Table 2.32 Densities (number $/ \mathrm{m}^{2}$ ) of Bromus rubens on Beatley Plots 3 and 4 in Rock Valley (ROVO05 and ROV006), 1963-1988. Errors are seen.

\begin{tabular}{ccc}
\hline & $\underline{\text { Plot 3 }}$ & $\underline{\text { Plot 4 }}$ \\
\cline { 2 - 3 } 1963 & 5.0 & 0.0 \\
1964 & 6.2 & 0.6 \\
1965 & 2.2 & 0.0 \\
1966 & 3.2 & 0.0 \\
1967 & 3.6 & 0.2 \\
1968 & 7.6 & 0.2 \\
1969 & $1 . .0$ & 0.0 \\
1970 & 19.8 & 1.0 \\
1971 & 0.2 & 0.0 \\
1972 & 0.0 & 0.0 \\
$\sim$ & $\sim 245 \pm 298$ & $\sim$ \\
1987 & $2034 \pm 632$ & - \\
1988 & & $514 \pm 158$ \\
& & \\
\hline
\end{tabular}


Table 2.33 Average weights (mg) of Bromus plants when growing at the same location.

Plot

Bromus rubens

Bromus tectorum

(Disturbed)

Mid Valley

Sedan $1000^{\prime}$

$26 \pm 11$

$23 \pm 9$

$9 \pm 2$

$-7$

Sedan $3000^{\prime}$

$11 \pm 3$

$19 \pm 6$

$17 \pm 4$

(Undisturbed)

Mid Valley

Sedan $5000^{\prime}$

$11 \pm 1$

T1 Control

$34 \pm 13$

$42 \pm 21$

$14 \pm 2$

$16 \pm 2$

$22 \pm 5$

$20 \pm 2$

$10^{*}$

$5^{*}$

$23 \pm 6$

$38 \pm 24$

$20^{*}$

* Only one value is given; no error term is calculable. 
Table 2.34 Numbers of ephemeral species and percent introduced biomass on sites where ephrmerals were sampled in 1988.

\begin{tabular}{|c|c|c|c|}
\hline Site & Location & $\begin{array}{l}\text { Number of } \\
\text { Species }\end{array}$ & $\begin{array}{l}\text { Percent } \\
\text { Introduced }\end{array}$ \\
\hline \multicolumn{4}{|l|}{ Disturbed } \\
\hline YUF009 & T1 GZ & 12 & 99.8 \\
\hline YUF014 & $\mathrm{T} 2 \mathrm{GZ}$ & 8 & 84.2 \\
\hline FRFO02 & Roadside & 26 & 5.2 \\
\hline MERO02 & Gopher & 12 & 97.6 \\
\hline YUF016 & Sedan $1000^{\prime}$ & 15 & 77.0 \\
\hline YUF017 & Sedan $3000^{\prime}$ & 22 & 98.8 \\
\hline YUF011 & 3B Scraped & 2 & 100. \\
\hline MIDO01b Burned & & 23 & 83.7. \\
\hline means & & 15.0 & 80.8 \\
\hline \pm sem & & \pm 2.9 & \pm 11.2 \\
\hline \multicolumn{4}{|l|}{ Undisturbed } \\
\hline JAF001 & Baseline & 13 & 10.2 \\
\hline ROVOO5 & Beatley 3 & 38 & 97.5 \\
\hline ROV006 & Beatley 4 & 29 & 65.2 \\
\hline FRF001a Baseline & & 32 & 41.0 \\
\hline FRF001b Baseline & & 20 & 39.5 \\
\hline FRF002C Control & & 31 & 18.9 \\
\hline YUF001a Baseline & & 21 & 88.8 \\
\hline YUF001b Baseline & & 15 & 98.0 \\
\hline YUF010 & T1 Control & 16 & 87.5 \\
\hline XUF015 & T2 Control & 20 & 98.0 \\
\hline YUF018 & Sedan $5000^{\prime}$ & 23 & 88.3 \\
\hline YUF012 & 3B Control & 30 & 96.1 \\
\hline MIDO01a Burn Control & & 23 & 84.0 \\
\hline PAM001 & Baseline & 16 & 0.0 \\
\hline RAMO01 & Baseline & 16 & 0.0 \\
\hline means & & 22.9 & 60.9 \\
\hline \pm sem & & \pm 1.9 & \pm 9.9 \\
\hline
\end{tabular}


Table 2.35 Densities of Bromus rubens and native species censused in several close plots in Rock Valley, 1963-1988.

\begin{tabular}{ccc}
\hline Year & Bromus & Native \\
& & \\
\hline $1963^{1}$ & 5.0 & 10.0 \\
1964 & 6.2 & 27.6 \\
1965 & 2.2 & 2.4 \\
1966 & 3.2 & 69.6 \\
1967 & 3.6 & 7.0 \\
1968 & 7.6 & 99.6 \\
1969 & 14.0 & 109.8 \\
1970 & 19.8 & 14.0 \\
1971 & 0.2 & 2.0 \\
1972 & 0.0 & 3.0 \\
1973 & 0.4 & 118. \\
1974 & 11.2 & 101. \\
1975 & 13.0 & 216. \\
1976 & 90.9 & 327. \\
$\sim$ & $\sim$ & $\sim$ \\
1983 & 89. & 108. \\
1984 & 167. & 19. \\
1985 & 156. & 111. \\
1986 & 745. & 98. \\
1987 & 2045. & 106. \\
1988 & & \\
\hline & & \\
\hline
\end{tabular}

'Sources: 1963-1972, data of lanice Beatley (Plot 3) held by P. A. Medica; 1973-76 data of F. B. Turner, Rack Valley Validation Site Reports, U. S. IBP/Desert Biome; 1983.5 data of R. B. and K. B. Hunter, unpublished; 1987-88 BECAMP annual reports. 


\section{REFERENCES}

Allen, E. B., and D. H. Knight. 1984. The effects of introduced annuals on secondary succession in sagebrush-grassland, Wyoming. SW Natur. 29(4):407-422.

Beatley, J. C. 1965. Ecology of the Nevada Test Site. II. Status of introduced species. UCLA Report 12-554. 39pp.

1966. Winter annual vegetation following a nuclear detonation in the Northern Mojave Desert (Nevada Test Site). Radiation Botany 6:69-82.

. 1973. Russian thistle (Salsola) species in western United States. J. Range Manage. 26:225-226.

- 1974. Phenological events and their environmental triggers in Mojave Desert ecosystems. Ecology 5:856-863.

Colman, E. A., and T. M. Hendrix. 1949. The fiberglass electrical soil moisture instrument. Soil Sci. 67:425-438.

Coville, F. V. 1893. Botany of the Death Valley expedition. Contrib. U. S. National Herbarium vol. IV. 363p.

Cronquist, A., A. H. Holmgren, N. H. Holmgren, J. L. Reveal, and P. K. Holmgren. 1977. Intermountain Flora: Vascular plants of the intermountain West, U.S.A. Vol Six. Colum. Univ. Press, New York.

Frenkel, R. E. 1979. Ruderal vegetation along some California Roadsides. Univ. of California Press. Berkeley. 163p.

Heil, G. W., M. J. A. Werger, W. de Mol, D. van Dam and B. Heijne. 1988. Capture of atmospheric ammonium by grassland canopies. Science 239:764-765.

Hunter, R. B., and P. D. Greger. 1986. Desert water balance using a combination of psychometric and resistance sensors. In Proceedings of the Rainfall Simulator Workshop, Jan. 14-15, 1985, L. J. Lane, (Ed.). Society for Range Management, 2760 W. Fifth Ave., Denver, CO 80204. pp. 30-34.

E. M. Romney, J. W. Childress and J. E. Kinnear. 1975. Responses and interactions in desert plants as influenced by irrigation and nitrogen applications. US/IBP Desert Biome Res. Memo. 75-13. 14p. 
E. M. Romney, A. Wallace, H. O. Hill, T. A. Ackerman and J. E. Kinnear. 1976. Responses and interactions in desert plants as influenced by irrigation and nitrogen applications. US/IBP Desert Biome Res. Memo. 76-14. 7p.

E. M. Romney, J. E. Kinnear, T. L. Ackerman, and H. O. Hill. (Unpublished manuscript). Responses and interactions in desert plants as influenced by irrigation and applications. Report of 1976 progress. Manuscript in BECAMP Biological Data Repository. $43 p$.

and P. A. Medica. 1989. Status of the Flora and Fauna on the Nevada Test Site in 1987. Dept. of Energy Report DOE/NV/10630-2. NTIS, U. S. Dept. of Commerce, Springfield, VA 22161. 103p.

Janzen, D. H. 1986. The eternal external threat. Pp. 286-303 in M. E. Soule (Ed.), Conservation Biology: the science of scarcity and diversity. Sinauer Associates, Inc. Sunderland, Massachusetts.

Kartesz, J. T. and R. Kartesz. 1980. in synonomized checklist of the vascular flora of the United States, Canada, and Greenland. Univ. No. Carolina Press, Chapel Hill, NC. 500 pp.

Mack, R. N. 1981. Invasion of Bromus tectorum L. into western North Arnerica: an ecological chronicle. Agro-Eycosystems 7(2):145-165.

Munz, P. A. 1974. A Flora of Southern California. Univ. Calif. Press, Berkeley. 1086p.

Piemeisel, R. L. 1938. Changes in weedy plant cover on cleared sagebrush land and their prubable causes. U. S. Dept. of Agric. Tech. Bull. 654. 44p.

1951. Causes affecting change and rate of change in a vegetation of annuals in Idaho. Ecology 32:53-72.

Rickard, W. H. and R. H. Sauer. 1982. Self-revegetation of disturbed ground in the deserts of Nevada and Washington. Northw. Sci. 56(1):41-47.

Shields, L. M., P. V. Wells, and W. H. Rickard. 1963. Vegetational recovery on atomic target areas in Nevada. Ecology 44(4):697-705.

Turner, F. B. and D. C. Randall. 1989. Net production by shrubs and winter annuals in Southern Nevada. J. Arid Environ. 17(1):23-26. 
Wallace, A., E. M. Romney, with Collaborators. Radioecology and ecophysiology of desert plants at the Nevada Test Site. TID-25954, NTIS, U. S. Dept. of Commerce, Springfield, VA 22151. 439p.

Went, F. W. and M. Westergaard. 1949. Ecology of desert plants. III. Development of plants in the Death Valley National Monument, California. Ecology 30(1):26-38. 


\title{
SECTION 3 \\ STATUS OF REPTILES IN 1988
}

\author{
by \\ Phillp A. Medica
}

\section{INTRODUCTION}

Reptile studies under the Basic Environmental Compliance and Monitoring Program (BECAMP) were initiated in 1987 to document the relative number or density of the common lizards at various locations on the Nevada Test Site (NTS), and to document changes which may occur over time. Desert tortoise growth studies initiated in Rock Valley (NTS) are being continued, and free-ranging tortoises throughout NTS will be enumerated on an ongoing basis.

The BECAMP reptile sampling project in 1988 included surveys of natural populations of lizards in three major valleys (Jackass Flats, Frenchman Flat, and Yucca Flat) and on Pahute Mesa. The resident reptile species on the BECAMP baseline monitoring plots were sampled to provide data on species composition and relative density, which eventually will provide information on the stability and condition of the populations under baseline conditions. Uta sampling was conducted on seven subsidiary plots in Yucca Flat in 1988 (T1 Blast area and control; T3 Blast area, 3B Consolidation Site and control; and a natural burn area and nearby unburned area).

Desert tortoises (Gopherus agassizii) were recaptured in Rock Valley, maintaining records on animals first marked in the early 1960s (Medica et al. 1975; Turner et al. 1987). As part of the BECAMP studies at the NTS, the tortoises in the Rock Valley study area wili be recaptured at least yearly. Tortoises were also searched for, marked and released in Frenchman Flat, northern Jackass Flats, Mercury Valley and Rock Valley.

\section{METHODS}

\section{SAMPLING LOCATIONS AND DATES}

Baseline monitoring plots $300 \times 300 \mathrm{~m}$, with an inner grid of $165 \times 165 \mathrm{~m}$ ( $2.72 \mathrm{ha}$ ) for small mammal studies and a $105 \times 105 \mathrm{~m}$ (1.10 ha) area within the small marnmal grid for lizard studies, were established on Pahute Mesa (PAM001) and Rainier Mesa (RAM001) in 1988. Lizard transect lines $500 \mathrm{~m}$ long with stakes $50 \mathrm{~m}$ apart along each line were also established on the Pahute Mesa baseline monitoring plot. The geographic location of each plot sampled in 1988 is illustrated in Figure 3.1, and the design of the baseline monitoring plot is shown in Figure 3.2. Appendix 3A lists the plot designations and the Nevada State Grid Coordinates 


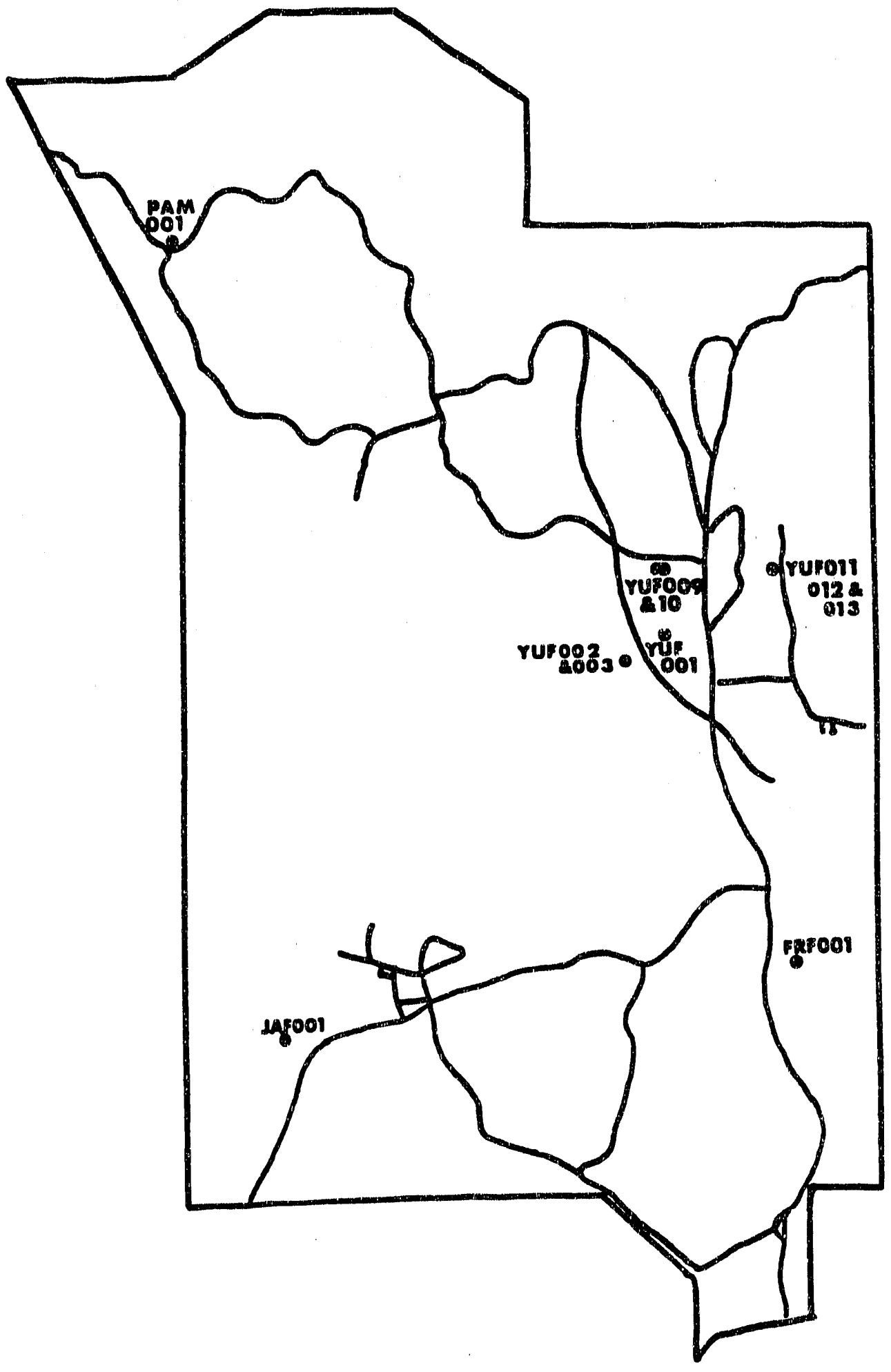

.

-

-

Figure 3.1 BECAMP study plots sampled for lizards in 1988. 
-

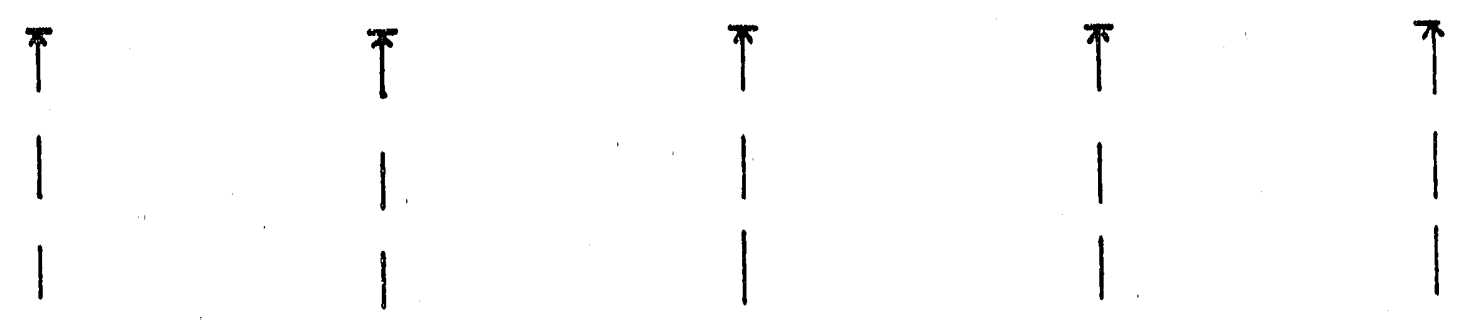

-

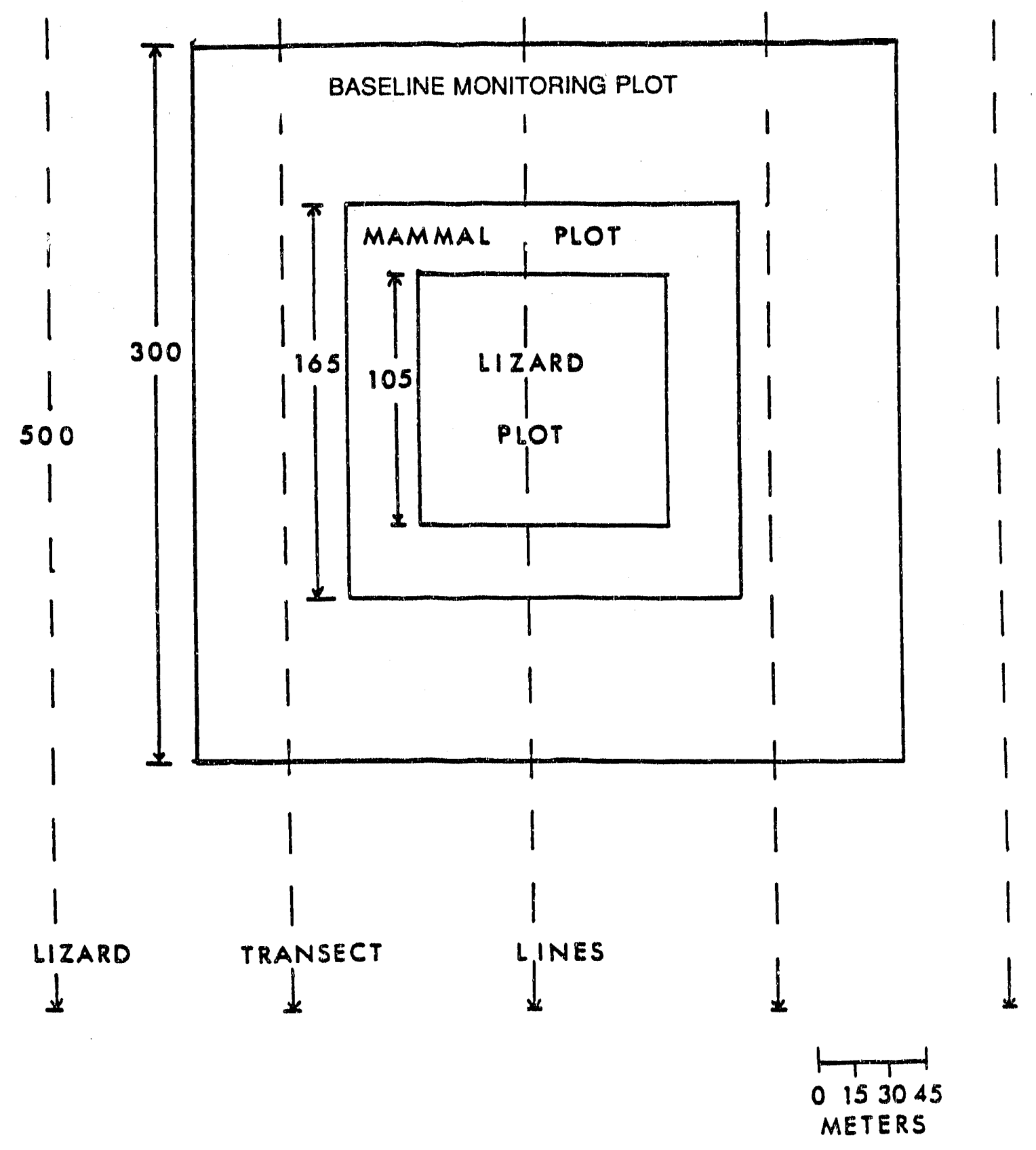

Figure 3.2 BECAMP pristine monitoring plot layout. 
for all plots established in 1987 and 1988. The lizard plots established on seven subsidiary sites in Yucca Flat were $75 \times 75 \mathrm{~m}(0.56 \mathrm{ha})$. Table 3.1 lists dates that the BECAMP lizard plots were sampled for Uta in 1988. The Pahute Mesa plot was not established soon enough to sample Uta in the spring. Uta sampling to estimate density was also conducted in the late summer (August 1-September 16) to obtain information on the number of hatchlings and surviving adults in eight study areas. Transect counts on four of the baseline monitoring plots for lizards other than Uta stansburiana were carried out between June 2 and June 30, 1988.

Table 3.1 Dates BECAMP study plots were sampled for Lta stansburiana in 1988.

\begin{tabular}{lllll}
\hline & \multicolumn{2}{c}{ Spring } & \multicolumn{2}{c}{ Summer } \\
\hline Plot & Started & Completed & Started & Completed \\
\hline JAFJ001 & March 4 & March 11 & August 1 & August 5 \\
FRF001 & March 14 & March 18 & August 22 & September 8 \\
YUF001 & March 21 & March 25 & August 8 & August 13 \\
PAM001 & - - & - & September 12 & September 16 \\
YUF002 & March 28 & April 5 & August 15 & August 26 \\
YUF003 & March 28 & April 5 & August 15 & August 26 \\
YUF009 & April 26 & April 29 & August 31 & September 6 \\
YUF010 & April 26 & April 29 & August 29 & September 6 \\
YUF011 & May 3 & May 18 & -- &.- \\
YUF012 & May 3 & May 18 & -- & - \\
YUF013 & May 3 & May 18 & -- & - \\
\hline
\end{tabular}

\section{LIZARD SAMPLING TECHNIQUES}

\section{Adult Uta: Baseline Monitoring Plots}

Uta stansburiana population densities were estimated during the spring (March) of 1988 on three baseline monitoring plots (JAF001, FRF001, and YUF001). Each plot was sampled during the early morning activity period for at least four days with two or three people capturing lizards by noosing. The density estimates used were from the final day of field sampling on which enough animals were recaptured to calculate an estimate (Appendix 3B).

All captured Uta were toe-clipped with a unique number so that individuals could be permanently identified (Figure 3.3). Lizards were painted with a unique paint pattern for short-term identification (Figures 3.4 and 3.5), weighed to the nearest $0.05 \mathrm{~g}$, measured 

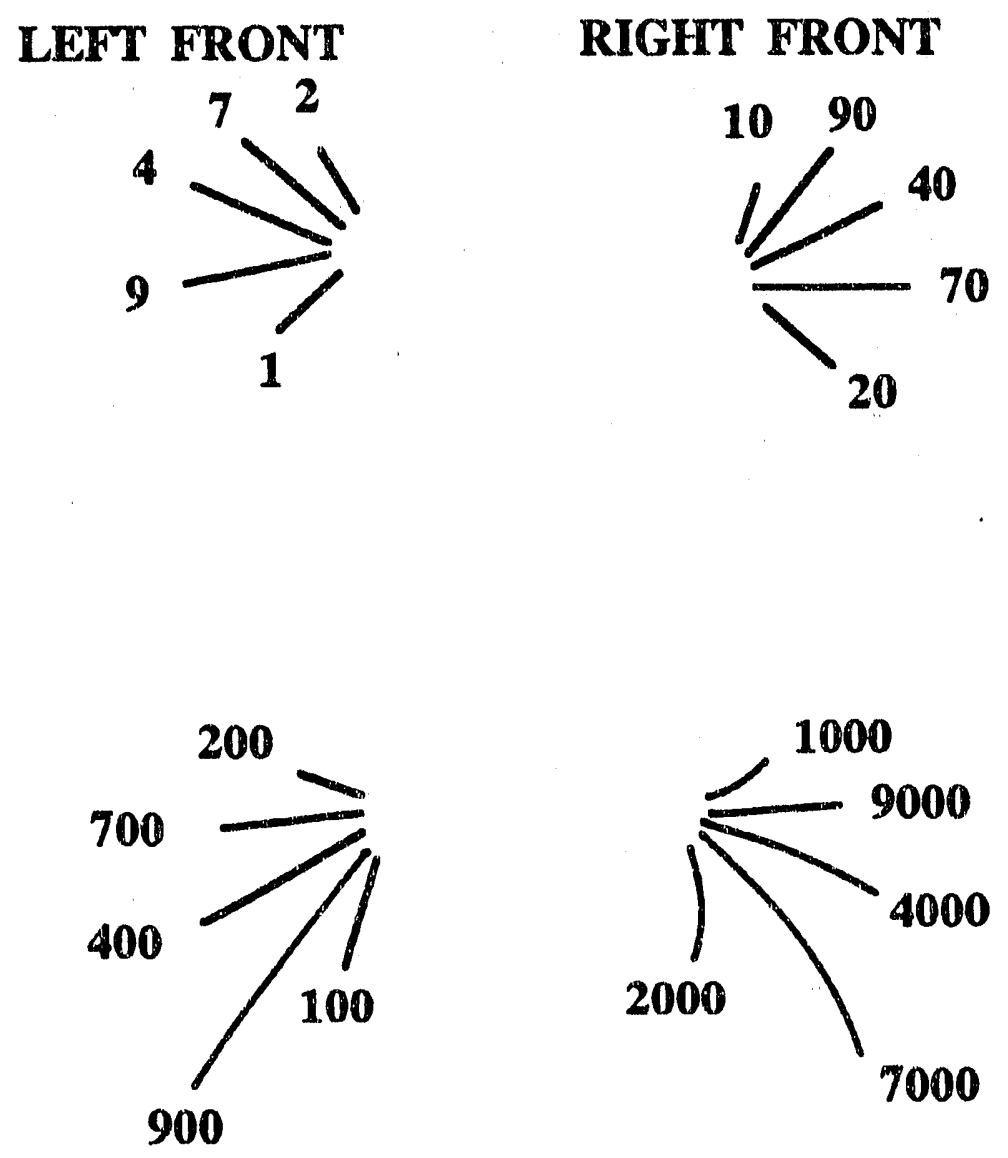

Figure 3.3 Dorsal view of lizard toes illustrating the toe numbering formula. 


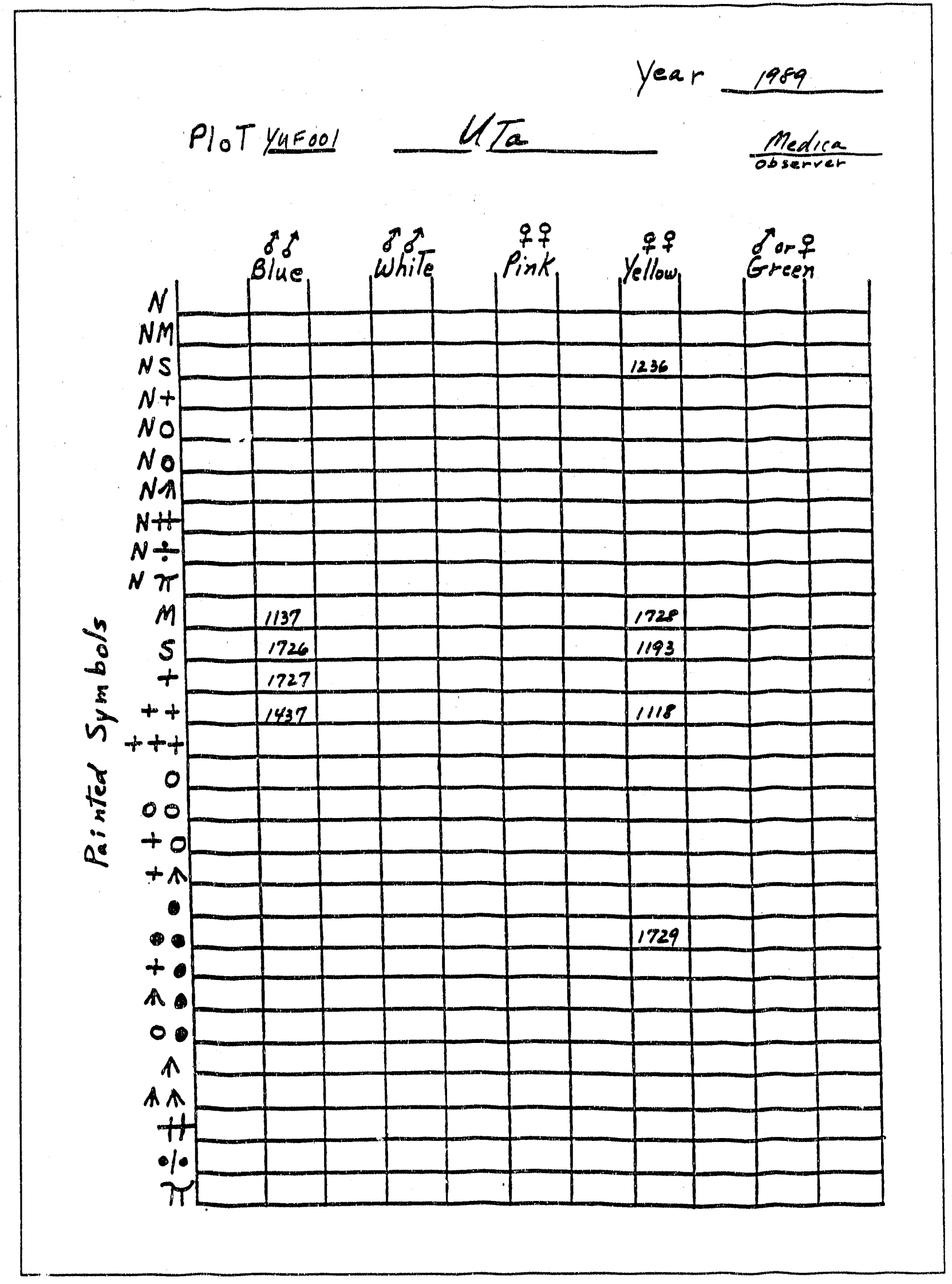

I igure 3.4 BECAMP lizard paint pattern form. 
9
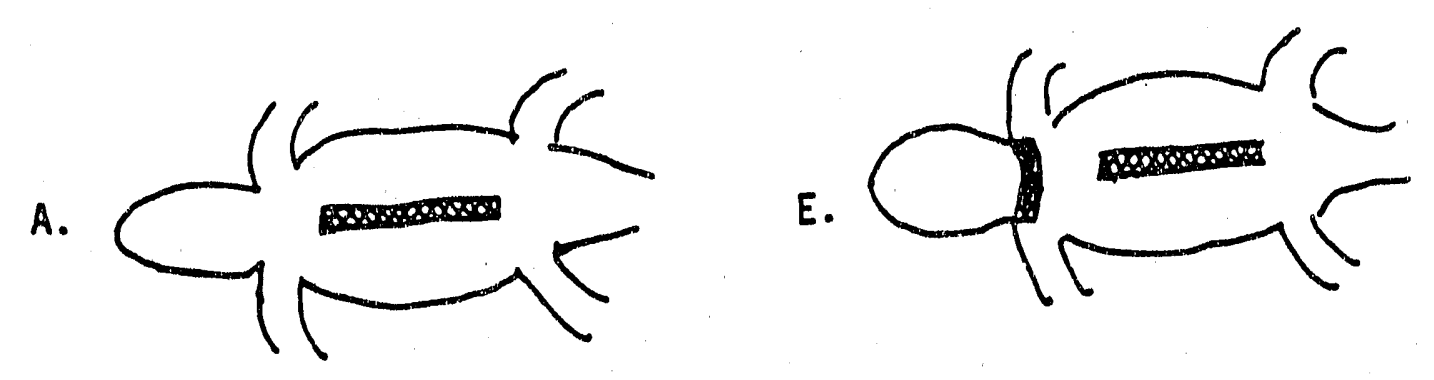

9

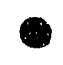
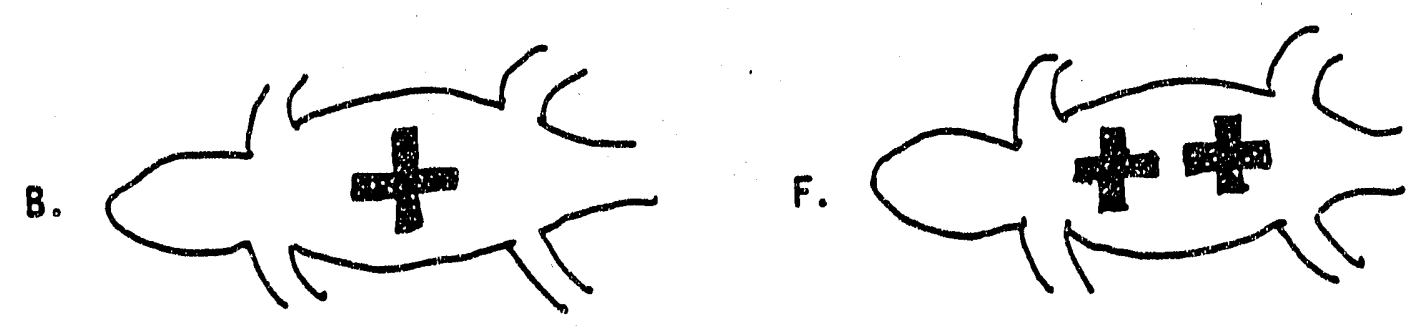

c.

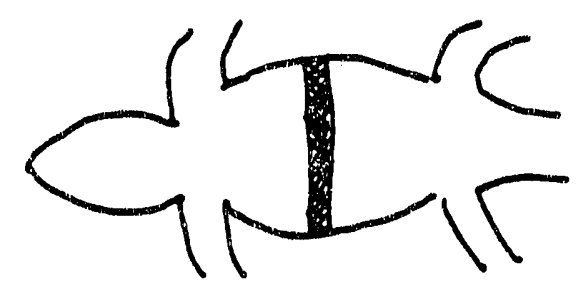

9
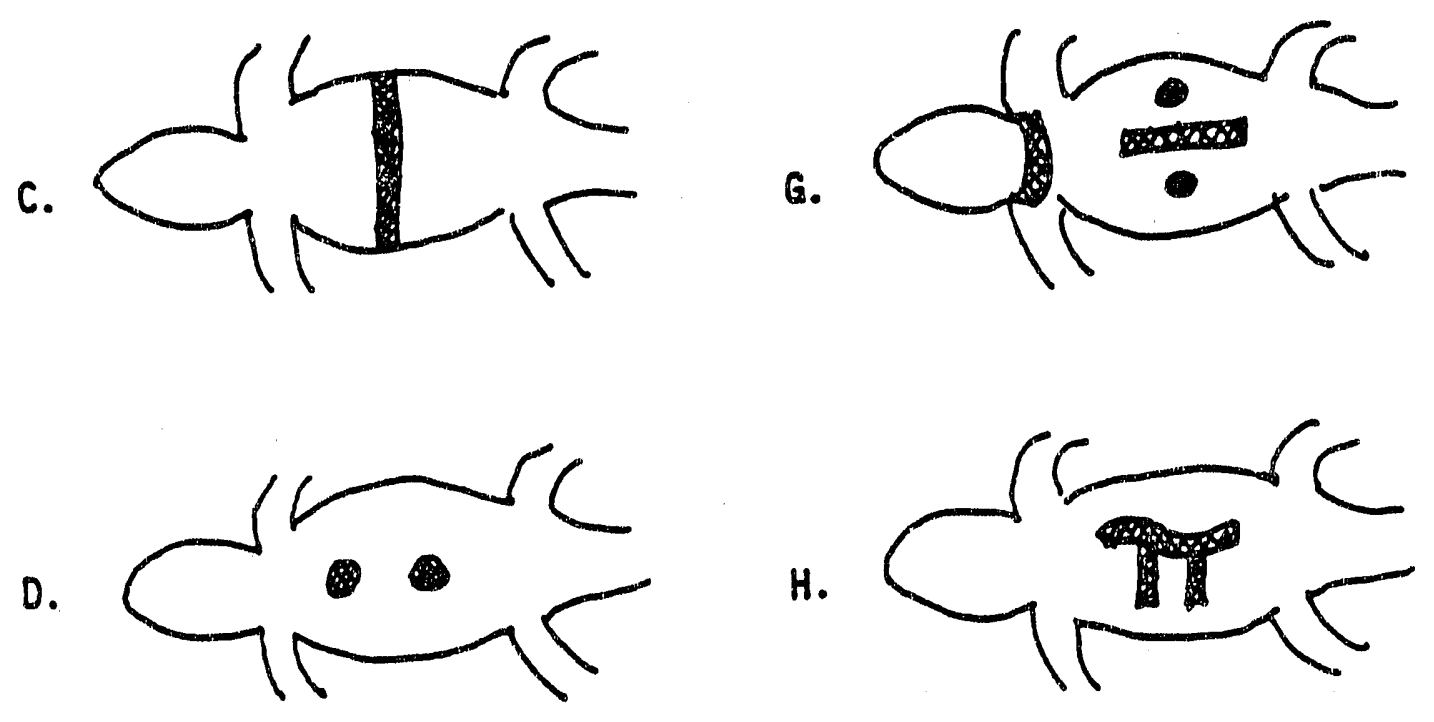
A. Is $S=$ Stripe
B. Is $+=$ Plus
C. Is $M=$ Midbody
D. Is $00=$ Dot Dot
E. Is NS $=$ Neck Stripe
F. Is $++=$ Plus Plus
G. Is $\mathbf{N}=$ Neck Division
$\mathrm{H}$. Is $=\mathrm{Pi}$

Figure 3.5 Symbols used to paint adult Uta. 
(snout-vent length in mm) and released where captured. Details of the techniques used and sample data sheets appear in Hunter and Medica (1989).

The Pahute Mesa baseline monitoring plot (PAM001) was sampled only in September 1988 when hatchlings as well as adults were enumerated. Estimates of adults surviving from March to the end of the reproductive season are based upon those marked individuals surviving the 5-month interval (March-August 1988) on the three baseline monitoring plots in the valleys.

\section{Hatchling Uta: Baselline Monltoring Plots}

Juvenile Uta were marked and released within four baseline monitoring plots. At lower elevations, the first hatchlings usually appear in mid-June (Medica and Turner 1976) and at higher elevations ' $\mathrm{n}$ mid-July (Tanner and Hopkin 1972). We began our hatchling sampling in Jackass Flats (JAF001) on August 1, 1988, and completed our Pahute Mesa plot (PAM001) on September 16, 1988.

Fisthling Uta were noosed within our study areas using the same techniques described for adult Uta (Hunter and Medica 1989). Hatchlings were toe-clipped, measured, sexed, weighed, and painted in the same manner as adult Uta.

\section{Line Transects (Onther Lizards on Basellne Monitoring Plots)}

Other species of lizards, including Cnemidophorus tigris, Callisaurus draconoides, Phrynosoma platyrhinos, and Gambelia wislizenii, were inventoried wing line transects. Measures of relative abundance can be used as an index of lizard species present and to provide a crude measurement of their density. Such line transect methods of counting lizards do not reflect true density, as has been shown by Degenhardt (1966) and Medica et al. (1971). It has also been determined that adult lizards are more readily observable than juveniles. However, the line transect technique provides information that can be used to indicate trends in relative abundance when comparing data from year to year. It is more labor-efficient to count these species rather than to attempt to mark-recapture them.

Five transect lines, each $500 \mathrm{~m}$ long, with numbered lath stakes every $50 \mathrm{~m}$, were walked for five days in four baseline monitoring plots by three observers each day. Sampling n.rmally began when soil surface temperatures averaged approximately $35^{\circ} \mathrm{C}(\sim 0830 \mathrm{hrs}$.) ani continued until lizard activity ceased. One pass of the entire plot usually was completed pricr to cessation of lizard activity which occurred when soil temperatures approached $50^{\circ} \mathrm{C}$ (Medica, 1967).

\section{DESERT TORTOISE SAMPLING}

Desert tortoises were resampled within the three fenced Rock Valley enclosures again in 1988 , maintaining the $25+$ years of growth records on these marked individuals. In 1988 we 
searched the same areas in northwest Frenchman Flat that were sampled in 1987. We also searched areas south of Kiwi Mesa in western Jackass Flats and Pluto Valley. The areas searched included washes, caliche overhangs and certain dirt roads where desert tritoises have been observed frequently.

\section{RESULTS AND DISCUSSION}

\section{UTA SAMPLES}

\section{Baseline Monktoring Plots: Spring 1988}

During sampling of Uta stansburiana adults, a total of 217 adult Uta were enumerated on all three baseline plots combined. The sex ratio was 95 males to 122 females, significantly different from 1:1 ratio ( $x^{2}$ test, .05 level).

Spring Uta density estimates, based upon mark-recapture analysis (Seber 1973), indicate that Uta is most dense in Jackass Flats, slightly less dense in Yucca Flat and sparsest in Frenchman Flat (Table 3.2).

Table 3.2 Estimated densities (number of lizards per hectare \pm two standard errors) of Uta stanshurinna in the spring (March) of 1988 on three baseline monitoring plots (1.10 ha) on the Nevada Test Site. The number in parentheses is the total number of individuals enumerated.

\begin{tabular}{lccc}
\hline & $\begin{array}{c}\text { Jackass Wlats } \\
\text { (JAF001) }\end{array}$ & $\begin{array}{c}\text { Frenchman Flat } \\
\text { (FRF001) }\end{array}$ & $\begin{array}{c}\text { Yucca Flat } \\
\text { (YUF001) }\end{array}$ \\
\hline A.dult Lizards & $96.6 \pm 14.8$ & $33.4 \pm 2.2$ & $91.1 \pm 9.9$ \\
Male & $(40)$ & $(15)$ & $(40)$ \\
Female & $(51)$ & $(21)$ & $(50)$ \\
Total & $(91)$ & $(36)$ & $(90)$ \\
\hline
\end{tabular}

\section{Baseline Manitorling Plots: Sununer 1988}

Estimates of adult and hatchling density in late summex appear in Table 3.3. The Pahute Mesa plot had the highest estimated density of hatchlings. Yucca Flat possessed the highest density of adult Uta (Table 3.3).

\section{Survivorship Ot Adult Uta in 1989}

Survivorship of marked adult Uta from spring (March 1988) to late summer (AugustSeptember 1988) was highest in Yucca Flat (34.4\%) and lowest in Frenchman Flat (8.3\%). Data comparing survivorship between the three valley baseline monitoring plots appear in Table 3.4. 
Table 3.3 Estimated densities (number of lizards per hectare \pm two standard errors) of Uta stansburiana in summer (August-September) 1988 on four baseline monitoring plots (1.10 ha) on the Nevada Test Site. Numbers in parentheses are the total numbers of individuals enumerated.

\begin{tabular}{lcccc}
\hline & $\begin{array}{c}\text { Jackass Flats } \\
\text { (JAF001) }\end{array}$ & $\begin{array}{c}\text { Frenchman Flat } \\
\text { (FRF001) }\end{array}$ & $\begin{array}{c}\text { Yucca Flat } \\
\text { (YUF001) }\end{array}$ & $\begin{array}{c}\text { Pahute Mesa } \\
\text { (PAM001) }\end{array}$ \\
\hline Adult Lizards & $21.3 \pm 18.3$ & $3.2 \pm 1.6$ & $41.5 \pm 12.9$ & $28.3 \pm 11.3$ \\
Male & $(7)$ & $(2)$ & $(17)$ & $(8)$ \\
Female & $(4)$ & $(1)$ & $(19)$ & $(16)$ \\
Total & $(11)$ & $(3)$ & $(36)$ & $(24)$ \\
Hatchling Lizards & $134.7 \pm 81.1$ & $54.0 \pm 35.4$ & $100.7 \pm 33.6$ & $142.0 \pm 27.5$ \\
Male & $(25)$ & $(12)$ & $(39)$ & $(62)$ \\
Female & $(30)$ & $(16)$ & $(29)$ & $(55)$ \\
Total & $(55)$ & $(28)$ & $(68)$ & $(117)$ \\
\hline
\end{tabular}

Table 3.4 Number and percent survivorship of marked adult Uta stansburiana from spring (March 1988) to late summer (August - September 1988) on three baseline monitoring plots (1.10 ha) on the Nevada Test Site.

\begin{tabular}{lcccccc} 
& \multicolumn{2}{c}{ JAF001 } & \multicolumn{2}{c}{ FRFO01 } & \multicolumn{2}{c}{ YUF001 } \\
& Spring & Summer & Spring & Summer & Spring & Summer \\
\hline $\begin{array}{l}\text { Adults } \\
\text { Males }\end{array}$ & 40 & 6 & 15 & 2 & 40 & 14 \\
Females & 51 & 4 & 21 & 1 & 50 & 17 \\
Total & 91 & 10 & 36 & 3 & 90 & 31 \\
$\begin{array}{l}\text { Percent } \\
\text { Survivorship }\end{array}$ & 11.0 & & 8.3 & & 34.4 \\
\hline
\end{tabular}

Ovenwinter Survhorship of luta

Overwinter survivorship of Uta marked as adults or hatchlings in 1987 and surviving to the spring of 1988 is listed by baseline monitoring plot in Table 3.5. Survivorship of adults was the highest in Jackass Flats (JAF001), intermediate in Yucca Flat (YUF001), and lowest in Frenchman Flat (FRFO01). The same trends were exhibited in the survivorship of hatchling Uta. It is interesting to note that Uta survivorship between March and August and annual plant densities (numbers per meter ${ }^{2}$ ) were highly correlated although annual biomass grams $/ \mathrm{m}^{2}$ was not (Table 3.6 ). 
Table 3.5 Number and overwinter survivorship (\%) of adult and juvenile Uta stansburiana on three baseline monitoring plots between August 1987 and March 1988.

\begin{tabular}{lcccccc}
\hline & \multicolumn{2}{c}{ Jackass Flats } & \multicolumn{2}{c}{ Frenchman Flats } & \multicolumn{2}{c}{ Yucca Flat } \\
& $\begin{array}{c}\text { August } \\
1987\end{array}$ & $\begin{array}{c}\text { March } \\
1988\end{array}$ & $\begin{array}{c}\text { August } \\
1987\end{array}$ & $\begin{array}{c}\text { March } \\
1988\end{array}$ & $\begin{array}{c}\text { August } \\
1987\end{array}$ & $\begin{array}{c}\text { March } \\
1988\end{array}$ \\
\hline Adult Lizards & & & & & & \\
$\quad$ Male & 7 & 4 & 5 & 2 & 16 & 8 \\
Female & 4 & 2 & 7 & 3 & 17 & 7 \\
Total & 11 & $6(54.5 \%)$ & 12 & $5(51.7 \%)$ & 33 & $15(45.5 \%)$ \\
Juvenile Lizards & & & & & & \\
Male & 20 & 5 & 27 & 5 & 57 & 11 \\
Female & 26 & 13 & 34 & 9 & 56 & 23 \\
Total & 46 & $18(39.1 \%)$ & 61 & $14(23.0 \%)$ & 113 & $34(30.1 \%)$ \\
\hline
\end{tabular}

Table 3.6 Survivorship of adult Uta stansburiana between March and August 1988 on the baseline monitoring plots compared to ephemeral biomass and numbers/meter'.

\begin{tabular}{llcc}
\hline Plot & $\begin{array}{c}\text { Uta } \% \\
\text { survivorship }\end{array}$ & $\begin{array}{c}\text { Ephemeral } \\
\text { number } / \mathrm{m}^{2}\end{array}$ & $\begin{array}{c}\text { Ephemeral } \\
\text { grams } / \mathrm{m}^{2}\end{array}$ \\
\hline JAF001 & 11.0 & $962 \pm 109$ & $10 \pm 1$ \\
FRF001 & 8.3 & $293 \pm 118$ & $21 \pm 11$ \\
YUF001 & 34.4 & $1713 \pm 431.5$ & $22 \pm 5$ \\
\hline
\end{tabular}

\section{Subsldlary Study Plots In Yucca Flat}

Burned and Unburned Study Areas. The burned and unburned plots (YUF002, burned; YUF003, unburned) were sampled in the spring of 1988 to determine any effect of the lack of adults surviving the reproductive season, as seen in our August 1987 sampling (Hunter and Medica, 1989). The spring adult Uta density estimates on the two 0.56-ha plots in 1988 were not significantly different, YUF002 $53.9 \pm 7.4 / \mathrm{ha}$ and YUF003 $59.1 \pm 4.6 / \mathrm{ha}$, indicating that the number of adults remaining on the plot in the sumiter of 1987 had little if any effect upon the number of animals ultimately recorded in the spring of 1988.

Summer adult density estimates in August (August 15-26) for YUF002 and YUF003 were 8.9 $\pm 4.1 /$ ha and $30.6 \pm 18.5 /$ ha respectively, with survivorship being $14.3 \%$ in YUF002 and 
22.6\% in YUF003. Hatchling density estimates were high in YUF003 with large standard error (Table 3.7). The trend exhibited in 1987 in the burned study plot (Hunter and Medica, 1989) continued in 1988, with red ad survivorship of adults in summer, suggesting that predation might be the cause of $t$ reduction of adults from spring to late summer.

Table 3.7 Estimated densities (number per hectare \pm two standard errors) of Uta stansburiana on subsidiary ( $0.56 \mathrm{ha}$ ) study plots YUF002 (burned) and YUF003 (unburned) in spring and summer of 1988. Numbers in parentheses are actual numbers of individuals enumerated.

\begin{tabular}{lcccc} 
& \multicolumn{2}{c}{ Spring 1988} & \multicolumn{2}{c}{ Summer 1988 } \\
& (YUF002) & (YUF003) & (YUF002) & (YUF003) \\
\hline Adult Lizards & $53.7 \pm 7.4$ & $58.8 \pm 9.1$ & $8.9 \pm 5.0$ & $30.2 \pm 18.5$ \\
Male & $(10)$ & $(9)$ & $(0)$ & $(2)$ \\
Female & $(18)$ & $(22)$ & $(4)$ & $(9)$ \\
Total & $(28)$ & $(31)$ & $(4)$ & $(11)$ \\
Hatchling Lizards & & & $109.4 \pm 14.8$ & $215.5 \pm 98.1$ \\
Male & & & $(24)$ & $(38)$ \\
Female & & & $(32)$ & $(21)$ \\
Total & & & $(56)$ & $(59)$ \\
\hline
\end{tabular}

In the fall of 1987, approximately equal amounts of effort were expended on each plot (13.7 man-hours on YUF002 and 13.3 man-hours on YUF003) with 4 Gambelia wislizenii enumerated on YUFO02 and $2 \underline{G}$. wislizenii on YUF003. G. wislizenii is a predator on Uta.

In August 1988 a comparable number of man-hours were spent sampling these plots (12.8 in YUF002, and 13.3 in YUF003) with $2 \mathrm{G}$. wislizenii hatchlings being captured in each plot. Based up in the equal sampling effort (total man-hours on YUF002, 26.5; and YUF003, 26.6) and nearly equal numbers of leopard lizards ( $G$. wislizenii) enumerated, it seems that the prey (Uta) were more easily preyed upon in the area (YUF002) that had been burned. On several occasions during the spring sampling, ravens were observed walking about the burned plot probably searching for food such as lizards, snakes or rodents. This was never observed in the unburned (YUF003) plot. Uta behavior was drastically different between the two plots as well. The lizards on the burned plot sought shelter in rodent burrows, as there was virtually no cover (1\%) (Hunter and Medica, 1989) on YUFO02. The Ula on the unburned plot (YUFO03), which had 23\% cover, sought shelter in the dense Stipa speciosa clumps and under shrubs. 
Blast Area Study at T1. Uta sampling was conducted at blast areas on sites of aboveground nuclear tests conducted between 1952 and 1957 (Figure 3.6). These areas were created by large fireballs enveloping the ground surface, denuding the vegetation, fusing portions of the sand and irradiating the area. Annual plant invasion took place rather rapidly (Shields and Wells, 1963\%. Otar study plot (YUF009) was located within the old disturbed area described by Trivier/and Jorgensen (1963:28-29) along the southeast transect line (3168 ft) from ground zerc at 1 ' 1 , In 1988 this region was characterized by dense cover of introduced annuals in spring and the lack of much shrub cover except for an occasional Atriplex or Chrysothamnus shrub. The control plot was established along this same transect line $(5808 \mathrm{ft})$ southeast of ground zero in the undisturbed vegetation in the Grayia. Lycium community. A region similar to that described by Tanner and Jorgensen (1963) is shown in Figure 2.2 this report.

A total of 61 adult Uta were enumerated on the T1 control area (YUF010) in the spring of 1988, compared to only 4 Uta adults on the T1 blast area plot (YUF009). There was $31 \%$ survivorship (19 of 61) of Uta aduits from spring to summer on YUFD10, compared to $0 \%$ survivorship on the blast area (YUF009). The estimated adult density on the blast area was $12.4 \pm 12.3$ compared to $121.7 \pm 15.0$ per hectare on the control plot. Correspondingly, the number of hatchling Uta enumerated on the blast area was 3 compared to 82 on the control site (Table 3.8).

\begin{tabular}{|c|c|c|c|c|c|}
\hline \multirow{3}{*}{\multicolumn{2}{|c|}{$\begin{array}{l}\text { istim } \\
\text { tansb } \\
\text { CUFO } \\
\text { ctual }\end{array}$}} & \multicolumn{4}{|c|}{$\begin{array}{l}\text { istimated densities (number per hectare } \pm \text { two standard errors) of Uta } \\
\text { tansburiana in late April and August-September on subsidiary (0.56 ha) plots } \\
\text { UF009 (T1 Blast Area) and YUF010 (Control). Numbers in parentheses are } \\
\text { ctual numbers of individuals enumerated. }\end{array}$} \\
\hline & & \multicolumn{2}{|c|}{ Spring 1988} & \multicolumn{2}{|c|}{ Summer 1988} \\
\hline & & (YUFO09) & (YUF010) & (YUF009) & (YUF010) \\
\hline Adult Lizard & & $12.4 \pm 12.3$ & $121.7 \pm 15.0$ & & $24.3 \pm 4.0$ \\
\hline Male & & (1) & (27) & $(0)$ & (13) \\
\hline Female & & (3) & (34) & $(0)$ & (12) \\
\hline Total & & (4) & $(61)$ & $(0)$ & (25) \\
\hline \multicolumn{3}{|c|}{ Hatchling Lizards } & & & $219.1 \pm 1.7$ \\
\hline Male & & & & (24) & (38) \\
\hline Fiemale & & & & (32) & (21) \\
\hline Total & & & & $(56)$ & (59) \\
\hline
\end{tabular}

Blast, Bladed and Undlsturbed Plots in Area 3. A third set of experimental study areas consisted of a ground-zero blast area (T3a, YUF013) a bladed area (the site of a previous contaminated waste dump, 3B, YUF011); and a control area in relatively undisturbed habitat consisting of mixed desert shrubs, YUFD12. Data based on capture-recapture of Uta 


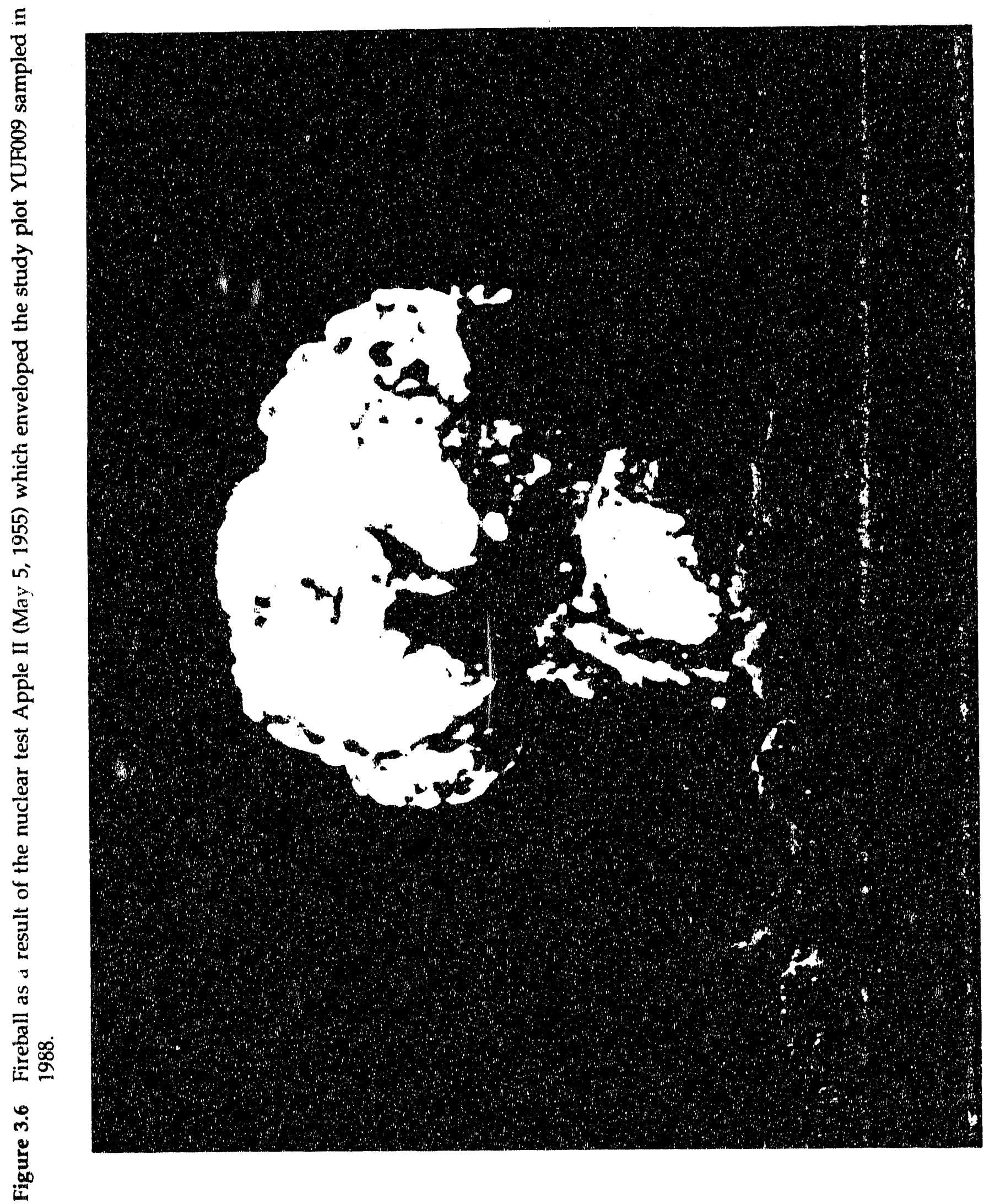

잉

0

D

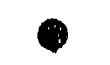

○

8 
stansburiana indicated that the control area possessed more Uta aduits. The 3B Consolidation Site (YUF011) which was bladed in the fall of 1987 was approximately 4.5 ha. No Uta was ever observed on the 3B Consolidation Site. The highest Uta density observed was on the undisturbed study plot (YUF012), which was approximately $150 \mathrm{~m}$ south of the 3B Consolidation Site (Table 3.9).

Table 3.9 Estimated densities (number per hectare \pm two standard errors) of Uta stansburiana in spring (early May) on subsidiary (0.56 ha) plots YUF011 (3B consolidation site), YUF012 (Undisturbed), and YUF013 (T3a Blast Area). Numbers in parentheses are actual numbers of individuals enumerated.

\begin{tabular}{lccc}
\hline & & Spring 1988 & \\
& YUF011 & YUF012 & YUF013 \\
\hline Adult Lizards & 0 & $80.0 \pm 53.9$ & $38.2 \pm 13.5$ \\
Male & $(0)$ & $(13)$ & $(7)$ \\
Female & $(0)$ & $(12)$ & $(10)$ \\
Total & $(0)$ & $(25)$ & $(17)$ \\
\hline
\end{tabular}

\section{LIZAAD TRANSE( IS ( LASTELINE MONITORING PLOTS)}

Estimated densities of Cnemidophorus tigris in 1988 ranged from a low of $.05 /$ ha on Pahute Mesa, to a high of $4.7 /$ ha in Jackass Flats (Table 3.10).

Table 3.10 Relative abundance of adult lizards observed on the BECAMP baseline monitoring plot transect lines surveyed in 1988. Estimated numbers/ha in parentheses.

\begin{tabular}{llclc}
\hline Species & $\begin{array}{c}\text { Jackass Flats } \\
\text { (JAF001) }\end{array}$ & $\begin{array}{c}\text { Frenchman Flat } \\
\text { (FRF001) }\end{array}$ & $\begin{array}{c}\text { Yucca Flat } \\
\text { (YUF001) }\end{array}$ & $\begin{array}{c}\text { Pahute Mesa } \\
\text { (PAM001) }\end{array}$ \\
\hline Cnemidophorus tigris & $1.43(4.71)$ & $.65(2.13)$ & $.68(2.25)$ & $.014(.05)$ \\
Callisaurus draconoides & $.006(.012)$ & $.201(.403)$ & $.03(.06)$ & 0 \\
Gambelia wislizenii & .13 & .15 & .052 & .021 \\
Phrynosoma platyrhinos & .024 & .041 & .030 & .007 \\
Crotaphytus collaris & 0 & 0 & 0 & .014 \\
Sceloporus occidentalis & 0 & 0 & 0 & .021 \\
Uta stansburiana & .089 & .30 & .36 & .43 \\
\hline
\end{tabular}

Comparing the transects sampled on the baseline monitoring plots in 1987 with the 1988 data indicated that there was a slight increase in whiptails on Frenchman Flat from 1.65/ha in 1987 to 2.13 in 1988, and a decrease in Yucca Flat from 6.37/ha in 1987 to 2.25 in 1988. At 
this point it is difficult to explain what may account for the decline of whiptail lizards in Yucca Flat. The number of lizards of the other species recorded were too few to accurately estimate density. It is evident (Table 3.3) that the transect method of sampling is inadequate to provide reasonable adult Uta abundance estimates. Multiplication factors from 10 to 100 would be required to arrive at density estimates comparable to those derived by markrecapture methods.

\section{REPTLE SPECIES OBSERVED ON THE NEVADA TEST STTE}

Tables 3.11 and 3.12 list the species of lizards, tortoise and snakes observed on the baseline monitoring plots in 1988 . The above tables also indicate species which, although not observed in 1988, were likely to be present on the plots based on records in published literature (Tanner and Jorgensen, 1963) or observations by the author in previous years.

On September 13, 1988, a noteworthy record for the western red-tailed skink Eumeces gilberti rubricaudatus) was recorded on Pahute Mesa off the southern end of our PAM001 plot. This was the third known record for this subspecies on the Nevada Test Site (Medica, Haworth and Kelly, 1990).

\section{DESERT TORTOISE STUDY}

Much of the southern portion of the NTS has been searched for the presence of the desert tortoise (Gopherus agassizii) by E.G.\& G. (O'Farrell, unpublished maps). In over 300 miles of line transects surveyed between 1984 and 1986, only seven desert tortoises were observed. In 1987 we walked $15(1.5 \mathrm{mi})$ transects mainly in northwest Frenchman Flat (one of the highest density tortoise locations on the NTS) and found only two tortoises. The above indicates that the use of the transect technique is not a very effective way to enumerate animals. The objective of our tortoise study was to enumerate as many animals as possible within the NTS and monitor their survivorship, growth, and movements, and to locate hibernacula.

A total of 48 different desert tortoises were captured on the NTS in 1988: 8 in Jackass Flats; 3 in Mercury Valley; 20 in Frenchman Flat; 17 in Rock Valley (14 within fenced plots, 3 free ranging) (Table 3.13).

Figure 3.7 illustrates the size relationships of the tortoises captured in 1987 and 1988, excluding those from fenced enclosures in Rock Valley.

\section{Rock Valley (Fenced Study Areas)}

In April 1988 a total of 14 desert tortoises were recaptured in the Rock. Valley study area: 11 of these animals were of known age ( \pm one year) (Turner, Medica and Bury, 1987). In September-October 1988 the Rock Valley study area was resampled and 9 tortoises were recaptured; all of these individuals had previously been captured in the spring except for No. 1211 which was last captured in October 1987. 
Growth in 1988 was measured on 8 tortoises (5 males and 3 females) first captured in April 1988 and then recaptured in September - October 1988. The mean growth and range was 1.6 $\mathrm{mm}(0-4 \mathrm{~mm})$ for male tortoises, and $2.7 \mathrm{~mm}(1-6 \mathrm{~mm})$ for females, with the overall mean of $2.0 \mathrm{~mm}$ growth in 1988. The one tortoise of undetermined sex which is known to be 24 years old grew $4 \mathrm{~mm}$ in 1988 which falls within the ranges observed for both male and female tortoises.

\section{Other Tortolses on the NTS}

A total of 34 additional desert tortoises were marked and released between April and October 1988 on the NTS. Three were from Rock Valley outside of the fenced plots, 18 from Frenchman Flat (14 from the northwestern portion of the valley and 4 from the southern part of the valley), 10 from Jackass Flats ( 8 from the northern end of the valley along Saddle Mountain Road and two from eastern Pluto Valley), and 3 from Mercury Valley just south and west of Mercury. Tortoise capture locations are shown on the map (Figure 3.8). 


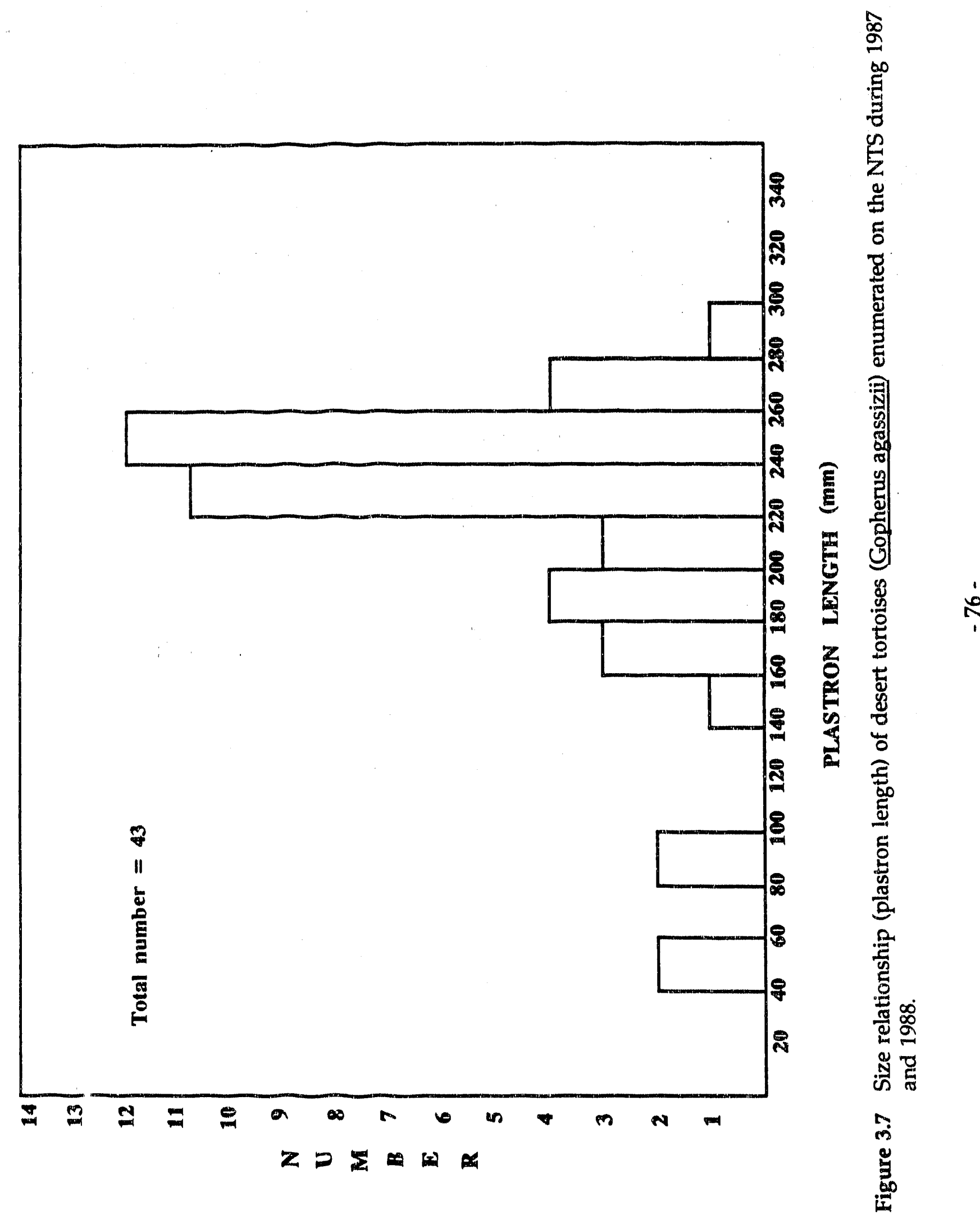


-

-

-

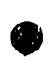

6

-

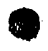

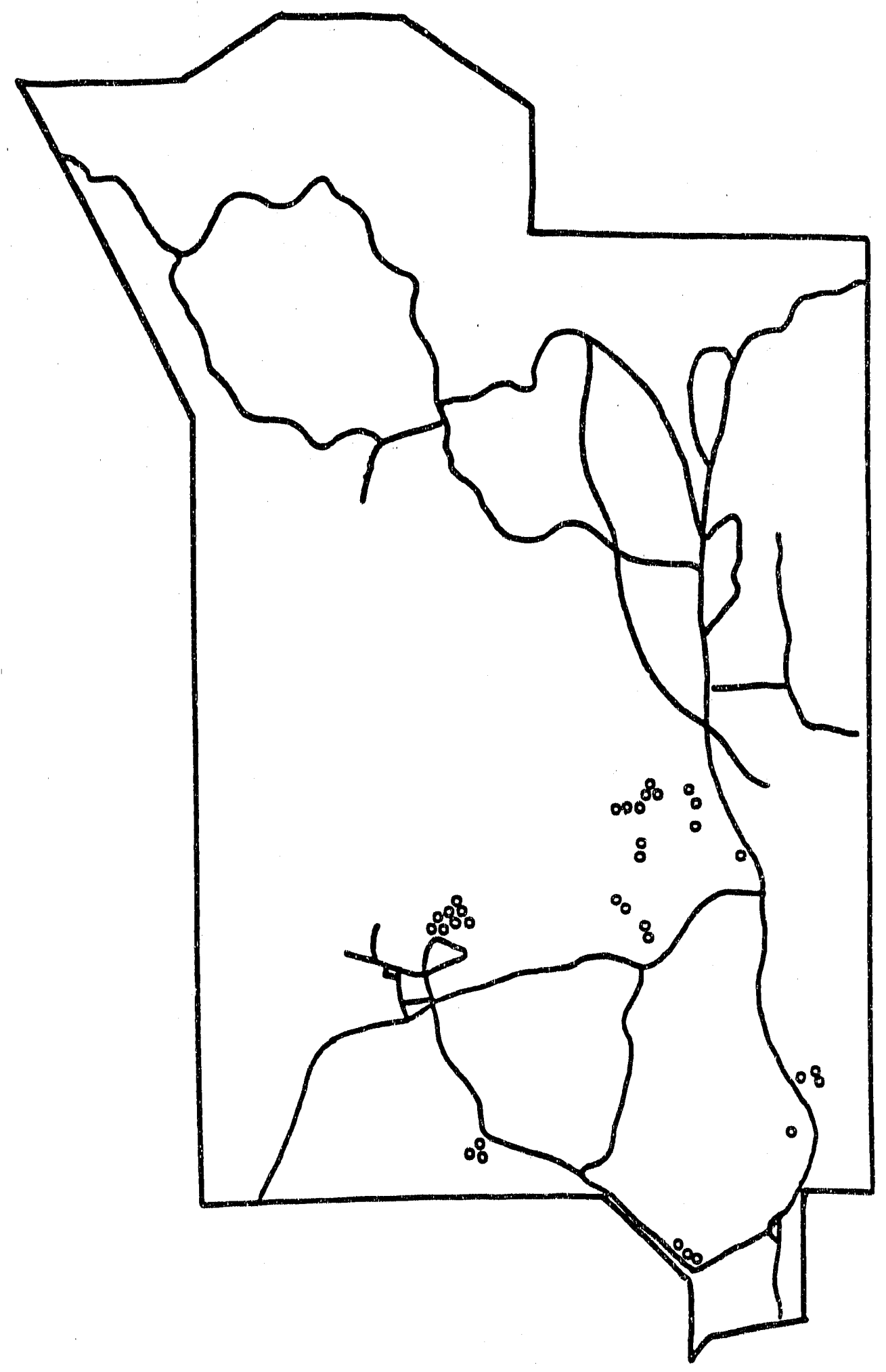

-

Figure 3.8 Tortoise capture locations on the NTS in 1988. 
Table 3.11 Lizards that were observed on the BECAMP baseline monitoring plots in 1988 are indicated by $(X)$; those which probably were present but were not observed $(P)$. Blanks indicate that the species probably does not occur on the plot.

\begin{tabular}{|c|c|c|c|c|c|}
\hline Species & $\begin{array}{c}\text { Jackass } \\
\text { Flats } \\
\text { (JAF001) }\end{array}$ & $\begin{array}{c}\text { Frenchman } \\
\text { Flat } \\
\text { (FRF001) }\end{array}$ & $\begin{array}{l}\text { Yucca Flat } \\
\text { (YUF001) }\end{array}$ & $\begin{array}{c}\text { Pahute } \\
\text { Mesa } \\
\text { (PAM001) }\end{array}$ & $\begin{array}{c}\text { Rainier } \\
\text { Mesa } \\
\text { (RAM001) }\end{array}$ \\
\hline Coleonyx variegatus & $\mathbf{P}$ & $\mathbf{P}$ & $x$ & & \\
\hline Callisaurus draconoides & $x$ & $x$ & $x$ & & \\
\hline Crotaphytus collaris & & & & $X$ & $P$ \\
\hline Gambelia wislizenii & $x$ & $x$ & $x$ & $x$ & \\
\hline Dipsosaurus dorsalis & $P$ & $x$ & & & \\
\hline Phrynosoma platyrhinos & $\mathrm{x}$ & $x$ & $x$ & $x$ & \\
\hline \multicolumn{6}{|l|}{ Sauromalus obesus } \\
\hline Sceloporus graciosus & & & & $\mathbf{P}$ & $\mathbf{P}$ \\
\hline Sceloporus magister & $P$ & $P$ & $x$ & & \\
\hline Sceloporus occidentalis & & & & $x$ & $x$ \\
\hline Uta stansburiana & $x$ & $x$ & $x$ & $x$ & $\mathrm{x}$ \\
\hline Eumeces gilberti & & & & $x$ & $P$ \\
\hline Eumeces skiltoniarius & & & & & $P$ \\
\hline Cnemidophorus tigris & $x$ & $x$ & $x$ & $x$ & \\
\hline Xantusia vigilis & & & $\mathrm{P}$ & & \\
\hline
\end{tabular}


Table 3.12 Desert tortoise and snakes that were observed oit the BEC.AMP baseline monitoring plots in 1988 (X); those which probably were present but were not observed $(P)$, and species which probably did not occur (blank).

\begin{tabular}{|c|c|c|c|c|c|}
\hline Species & $\begin{array}{c}\text { Jackass } \\
\text { Flats } \\
\text { (JAF001) }\end{array}$ & $\begin{array}{c}\text { Frenchman } \\
\text { Flat } \\
\text { (FRF001) }\end{array}$ & $\begin{array}{c}\text { Yucca } \\
\text { Flat } \\
\text { (YUF001) }\end{array}$ & $\begin{array}{c}\text { Pahute } \\
\text { Mesa } \\
\text { (PAM001) }\end{array}$ & $\begin{array}{c}\text { Rainier } \\
\text { Mesa } \\
\text { (RAM001) }\end{array}$ \\
\hline Gopherus agassizii & $P$ & $P$ & & & \\
\hline Arizona elegans & $P$ & P & & & \\
\hline Chionactis occipitalis & $x$ & $P$ & $\mathbf{P}$ & & \\
\hline Diadophis punctatus & & & & $\mathrm{P}$ & $P$ \\
\hline Hypsiglena torquata & $P$ & $P$ & $\mathbf{P}$ & & \\
\hline Lampropeltis getulus & $\mathbf{P}$ & $\mathrm{P}$ & $\mathbf{P}$ & $P$ & $\mathrm{P}$ \\
\hline Leptotyphlops humilis & $P$ & $P$ & & & \\
\hline Masticophis flagellum & $\mathbf{P}$ & $\mathrm{P}$ & $\mathbf{P}$ & $P$ & \\
\hline Masticophis taeniatus & & & & $\mathrm{P}$ & $\mathbf{P}$ \\
\hline Phyllorhynchus decurtatus & $P$ & $P$ & & & \\
\hline Pituophis melanoleucus & $\mathrm{P}$ & $P$ & $\mathbf{P}$ & $\mathbf{P}$ & $P$ \\
\hline Rhinocheilus lecontei & $P$ & $\mathrm{P}$ & $\mathbf{P}$ & $P$ & \\
\hline Salvadora hexalepis & $P$ & $P$ & $\mathbf{P}$ & & \\
\hline Sonora semiannulata & $P$ & $P$ & $\mathbf{P}$ & & \\
\hline Tantilla utahensis & $P$ & $\mathrm{P}$ & $\mathrm{P}$ & & \\
\hline Trimorphodon lambda & $P$ & $P$ & & & \\
\hline Crotalus cerastes & $x$ & $P$ & $\mathrm{P}$ & & \\
\hline Crotalus mitchelli & $P$ & $P$ & $\mathbf{P}$ & $\mathrm{P}$ & $P$ \\
\hline
\end{tabular}


Table 3.13 Desert tortoises (Gopherus agassizii) captured on the Nevada Test Site in 1988.

\begin{tabular}{|c|c|c|c|c|c|}
\hline & Arimal \# & Sex & $\begin{array}{c}\text { Plastron } \\
\text { length } \\
\text { (mm) }\end{array}$ & $\begin{array}{l}\text { Carapace } \\
\text { length } \\
(\mathrm{mm})\end{array}$ & $\begin{array}{l}\text { Weight } \\
\text { (g) }\end{array}$ \\
\hline \multirow[t]{8}{*}{ Jackass Flats } & 15 & $M$ & 210 & 228 & 2350 \\
\hline & 16 & $F$ & 193 & 213 & 1850 \\
\hline & 17 & $M$ & 208 & 222 & 2100 \\
\hline & 18 & $M$ & 254 & 278 & 4175 \\
\hline & 19 & F & 244 & 262 & 3375 \\
\hline & 20 & $\mathrm{~F}$ & 240 & 262 & 3425 \\
\hline & 21 & $\mathrm{~F}$ & 231 & 255 & 2925 \\
\hline & 23 & $\mathrm{~F}$ & 228 & 254 & 3100 \\
\hline \multirow[t]{20}{*}{ Frenchman Flat } & 11 & $M$ & 253 & 265 & 3900 \\
\hline & 12 & $F$ & 248 & 262 & 3575 \\
\hline & 13 & $F$ & 249 & 267 & 3700 \\
\hline & 14 & $F$ & 246 & 260 & 3550 \\
\hline & 24 & $F$ & 222 & 239 & 2725 \\
\hline & 25 & J & 143 & 150 & 650 \\
\hline & 26 & $\mathrm{~F}$ & 265 & 270 & 3725 \\
\hline & 27 & M & 228 & 237 & 2525 \\
\hline & 28 & $F$ & 218 & 231 & 2025 \\
\hline & 29 & $\mathrm{~F}$ & 236 & 253 & 2950 \\
\hline & 32 & $M$ & 198 & 202 & 1850 \\
\hline & 33 & $\mathbf{M}$ & 195 & 212 & 1725 \\
\hline & 34 & $M$ & 195 & 206 & 1775 \\
\hline & 35 & $\mathrm{H}$ & 45 & 48 & $20-25$ \\
\hline & 36 & $\mathrm{H}$ & 45 & 48 & $20-25$ \\
\hline & 37 & $\mathbf{M}$ & 263 & 286 & 4850 \\
\hline & 38 & $M$ & 236 & 245 & 2875 \\
\hline & 41 & $M$ & 272 & 282 & 4225 \\
\hline & 42 & J & 100 & 110 & 300 \\
\hline & 43 & $\mathrm{M}$ & 261 & 289 & 4000 \\
\hline
\end{tabular}


Table 3.13 Desert tortoises (Gopherus agassizii) captured on the Nevada Test Site in 1988. (Continued)

\begin{tabular}{|c|c|c|c|c|c|}
\hline & Animal \# & Sex & $\begin{array}{l}\text { Plastron } \\
\text { length } \\
(\mathrm{mm})\end{array}$ & $\begin{array}{c}\text { Carapace } \\
\text { length } \\
\text { (mm) }\end{array}$ & $\begin{array}{c}\text { Weight } \\
\text { (g) }\end{array}$ \\
\hline \multirow[t]{3}{*}{ Mercury Valley } & 22 & $\mathrm{~F}$ & 176 & 199 & 1925 \\
\hline & 39 & $M$ & 236 & 245 & 2875 \\
\hline & 40 & $\mathrm{~F}$ & 186 & 207 & 1575 \\
\hline \multicolumn{6}{|l|}{$\begin{array}{l}\text { Rock Valley } \\
\text { (Fenced Plots)" }\end{array}$} \\
\hline \multirow[t]{3}{*}{ Plot 1} & 4111 & $M$ & 235 & 249 & 2750 \\
\hline & 4415 & M & 236 & 269 & 3600 \\
\hline & 2444 & $F$ & 218 & 226 & 2250 \\
\hline \multirow[t]{2}{*}{ Plot 2} & 1112 & $M$ & 247 & 278 & 3650 \\
\hline & 444 & $M$ & 234 & 254 & 3200 \\
\hline \multirow[t]{9}{*}{ Plot 3} & 1222 & $\mathrm{~F}$ & 211 & 222 & 2150 \\
\hline & 1411 & $F$ & 215 & 229 & 2425 \\
\hline & 2111 & $\mathrm{M}$ & 221 & 236 & 2450 \\
\hline & 4121 & J & 157 & 169 & 975 \\
\hline & 4414 & M & 226 & 252 & 2700 \\
\hline & 4811 & $\mathrm{~F}$ & 218 & 234 & 2500 \\
\hline & 5111 & $F$ & 218 & 231 & 2275 \\
\hline & 6111 & F & 237 & 242 & 2525 \\
\hline & 8222 & $M$ & 224 & 232 & 2250 \\
\hline \multirow{3}{*}{$\begin{array}{l}\text { Rock Valley } \\
\text { (Unfenced) }\end{array}$} & 10 & $M$ & 245 & 261 & 3025 \\
\hline & 30 & $M$ & 240 & 261 & 3075 \\
\hline & 31 & F & 164 & 185 & 1200 \\
\hline
\end{tabular}

"Spring capture weights and measurements only. 


\section{REFERENCES}

Degenhardt, W. G. 1966. A method of counting some diurnal ground lizards of the genus Holbrookja and Cnemidophorus with results from the Big Bend National Park. Amer. Midl. Nat. 75:61-100.

Hunter, R. B. and P. A. Medica. 1989. Status of the flora and fauna on the Nevada Test Site: Results of continuing Basic Environmental $R$ ssearch January through December 1987. U.S. Dept. of Energy DOE/NV/10630-2 pp " -103 .

Medica, P. A. 1967. Food habits, habitat preference, reproduction, and diurnal activity in four sympatric species of whiptail lizards (Cnemidophorus) in south central New Mexico. Bull. So. Calif. Acad. Sci. 66(4):251-276.

R. B. Bury, and F. B. Turner. 1975. Growth of the desert tortoise (Gopherus agassizi) in Nevada. Copeia: 1975(4):639-643.

O. L. Haworth, and M. S. Kelly. 1990. Geographic distribution: Eumeces gilberti rubricaudatus. Herpt. Rev. 21(2):40.

C. A. Hoddenbach, and J. R. Lannom, Jr. 1971. Lizard sampling techniques. Rock Valley Misc. Publ. No. 1 pp. 1-55.

and F. B. Turner. 1976. Reproduction by Uta stansburiana (Reptilia, Lacertilia, Iguanidae) in southern Nevada. Journal of Herpetology 10(2):123-128.

Seber, G. A. F. 1982. The estimation of animal abundance and related parameters. Macmillan, New York.

Shields, L. M. and P. V. Wells. 1963. Recovery of vegetation on Atomic Target areas at the Nevada Test Site. In: Schultz, V., and A. W. Klement, Jr. Radioecology, Proc. First Nat. Symp. on Radioecology. 307-310.

Tanner, W. W. and J. M. Hopkin. 1972. Ecology of Sceloporus occidentalis longipes Baird and Uta stansburiana stansburiana Baird and Girard on Rainier Mesa, Nevada Test Site, Nye County, Nevada. BYU Science Bull., Biol. Series 15(4):1-39.

and C. D. Jorgensen. 1963. Reptiles of the Nevada Test Site. BYU Science Bull., Biol. Series 3(3):1-31; 1 map.

Turner, F. B., P. A. Medica, and R. B. Bury. 1987. Age-size relationships of desert tortoises (Gopherus agassizi) in southern Nevada. Copeia 1987 (4):974-979. 
Nevada State

Plots Grid Coordinate

Area

5

FRF001

FRF002

N 751,745

E 699,304

N 749,465

E 693,519

JAF001

MER001

MER002

MER003

MID001

MIDO02

MID003

PAM001

RAM001

RED001

RED002
N 735,274

E 585,114

N 698,283

E 696,420

N 692,904

E 660,831

N 694,735

E 660,918

N 781,561

E 639,067

N 782,118

E 636,789

N 781,484

E 636,324

N 911,211

E 563,739

N 888,161

E 631,260

N 848,765

E 629,166

N 848,402

E 631,235
25

23

22

22

14

14

14

20

12

Rainier Mesa

17

Burned Area

(July 1988)

Redrock Valley

17

Redrock Valley 
Appendix 3A

(Continued)

\begin{tabular}{|c|c|c|c|}
\hline Plots & $\begin{array}{l}\text { Nevada State } \\
\text { Grid Coordinate } \\
\end{array}$ & Area & Location \\
\hline ROV001 & $\begin{array}{l}\text { N } 705,812 \\
\text { E } 639,433\end{array}$ & 25 & $\begin{array}{l}\text { Fenced Plot A } \\
\text { (UCLA) }\end{array}$ \\
\hline ROV002 & $\begin{array}{l}\text { N } 706,766 \\
\text { E } 641,167\end{array}$ & 25 & $\begin{array}{l}\text { Fenced Plot B } \\
\text { (UCLA) }\end{array}$ \\
\hline ROV003 & $\begin{array}{l}\text { N 705,169 } \\
\text { E } 638,582\end{array}$ & 5 & $\begin{array}{l}\text { Fenced Plot C } \\
\text { (UCLA.) }\end{array}$ \\
\hline ROV004 & $\begin{array}{l}\text { N } 706,979 \\
\text { E } 639,972\end{array}$ & 25 & $\begin{array}{l}\text { Unfenced Plot D } \\
\text { (UCLA) }\end{array}$ \\
\hline ROV005 & $\begin{array}{l}\text { N } 704,975 \\
\text { E } 640,393\end{array}$ & 25 & $\begin{array}{l}\text { Beatley Plot } 3 \\
\text { (UCLA) }\end{array}$ \\
\hline ROV006 & $\begin{array}{l}\text { N } 710,093 \\
\text { E } 644,831\end{array}$ & 25 & $\begin{array}{l}\text { Beatley Plot } 4 \\
\text { (UCLA) }\end{array}$ \\
\hline ROV007 & $\begin{array}{l}\text { N } 707,038 \\
\text { E } 639,531\end{array}$ & 25 & IBP Plot 16 \\
\hline YUF001 & $\begin{array}{l}\text { N } 822,135 \\
\text { E } 670,729\end{array}$ & 1 & Yucca Flat \\
\hline YUFO02 & $\begin{array}{l}\text { N } 819,572 \\
\text { E } 664,869\end{array}$ & 6 & Burn (June 1985) \\
\hline YUJF003 & $\begin{array}{l}\text { N } 818,445 \\
\text { E } 664,450\end{array}$ & 6 & Unburned Area \\
\hline YUF004 & $\begin{array}{l}\text { N } 838,631 \\
\text { E } 664,089\end{array}$ & 1 & $\begin{array}{l}\text { T1 Plots Romney UCLA } \\
\# 1\end{array}$ \\
\hline YUF005 & $\begin{array}{l}\text { N } 838,581 \\
\text { E } 663,589\end{array}$ & 1 & $\begin{array}{l}\text { T1 Plots Rornney LICLA } \\
\# 2\end{array}$ \\
\hline YUF006 & $\begin{array}{l}\text { N } 838,531 \\
\text { E } 663,089\end{array}$ & 1 & $\begin{array}{l}\text { T1 Plots Romney UCLA } \\
\# 3\end{array}$ \\
\hline
\end{tabular}


Appendix 3A

(Continued)

\begin{tabular}{|c|c|c|c|}
\hline Plots. & $\begin{array}{l}\text { Nevada State } \\
\text { Grid Coordinate }\end{array}$ & Area & Location \\
\hline YUF 007 & $\begin{array}{l}\text { N } 838,481 \\
\text { E } 662,489\end{array}$ & 1 & $\begin{array}{l}\text { T1 Plots Romney UCLA } \\
\# 4\end{array}$ \\
\hline YUFOD8 & $\begin{array}{l}\text { N } 838,481 \\
\text { E } 661,689\end{array}$ & 1 & $\begin{array}{l}\text { T1 Plots Romuney UCL.A } \\
\# 5\end{array}$ \\
\hline YUF009 & $\begin{array}{l}\text { N } 836,736 \\
\text { E } 666,833\end{array}$ & 1 & $\begin{array}{l}\text { T1 Blast Area } 3168 \mathrm{ft} . \\
\text { SE, GZ }\end{array}$ \\
\hline YUF010 & $\begin{array}{l}\text { N } 834,635 \\
\text { E } 668,974\end{array}$ & 1 & $\begin{array}{l}\text { T1 Undisturbed } 5808 \mathrm{ft} \text {. } \\
\text { SE, GZ }\end{array}$ \\
\hline YUF011 & $\begin{array}{l}\text { N } 838,553 \\
\text { E } 693,936\end{array}$ & 3 & 3B Consolidation Site \\
\hline YUF012 & $\begin{array}{l}\text { N } 837,648 \\
\text { E } 694,079\end{array}$ & 3 & 3B Undisturbed Area \\
\hline YUF013 & $\begin{array}{l}\text { N } 838,003 \\
\text { E } 692,355\end{array}$ & 3 & T3 Blast Area ESE, GZ \\
\hline YUF014 & $\begin{array}{l}\text { N } 869,364 \\
\text { E } 661,309\end{array}$ & 2 & T2-1 Plot \\
\hline YUF015 & $\begin{array}{l}\text { N } 872,679 \\
\text { E } 665,894\end{array}$ & 2 & T2-5 Plot \\
\hline YUF016 & $\begin{array}{l}\text { N } 884,782 \\
\text { E } 682,661\end{array}$ & 10 & $\begin{array}{l}\text { Sedan } 1500 \mathrm{ft} . \\
\text { NE, GZ } 16 \text { A Line }\end{array}$ \\
\hline YUF017 & $\begin{array}{l}\text { N } 885,787 \\
\text { E } 684,214\end{array}$ & 10 & $\begin{array}{l}\text { Sedan } 3500 \mathrm{ft} . \\
\text { NE, GZ } 16 \text { A Line }\end{array}$ \\
\hline YUJF018 & $\begin{array}{l}\text { N } 886,405 \\
\text { E } 687,088\end{array}$ & 10 & $\begin{array}{l}\text { Sedan } 5250 \mathrm{ft} . \\
\text { NE, GZ } 16 \text { A Line }\end{array}$ \\
\hline
\end{tabular}


Density Estimates for Adult Uta stansburlane Sampied in Spring and Summer, and Hatchlings Sampled During the Summer of 1988 on the BECAMP

Study Plots on the Nevada Test Site

The table heading JAF188ADUTASPRDEN stands for the following:

IAF $\quad=$ Jackass Flats.

$1=$ Plot 001 .

$88=$ year 1988.

ADUTA = Adult Uta.

SPRDEN = Spring dersity.

Lines 1-7 of each table mean the following:

N1 = Cumulative number of individuals recorded in previous days samples.

$\mathrm{XB}=$ Number of new individuals captured on day 2.

M2 = Number in N2 which were recaptured (marked in a previous sample).

N2 = Total number of individuals captured in the days sample.

$\mathrm{N}^{*} / \mathrm{HA}=$ Population density estimate in number per hectare.

$\mathrm{V} / \mathrm{HA} 2$ = Variance estimate per hectare squared.

$2 \mathrm{SE} / \mathrm{HA}=$ two times the standard error per hectare.

Calculations are based on Seber (1982). 


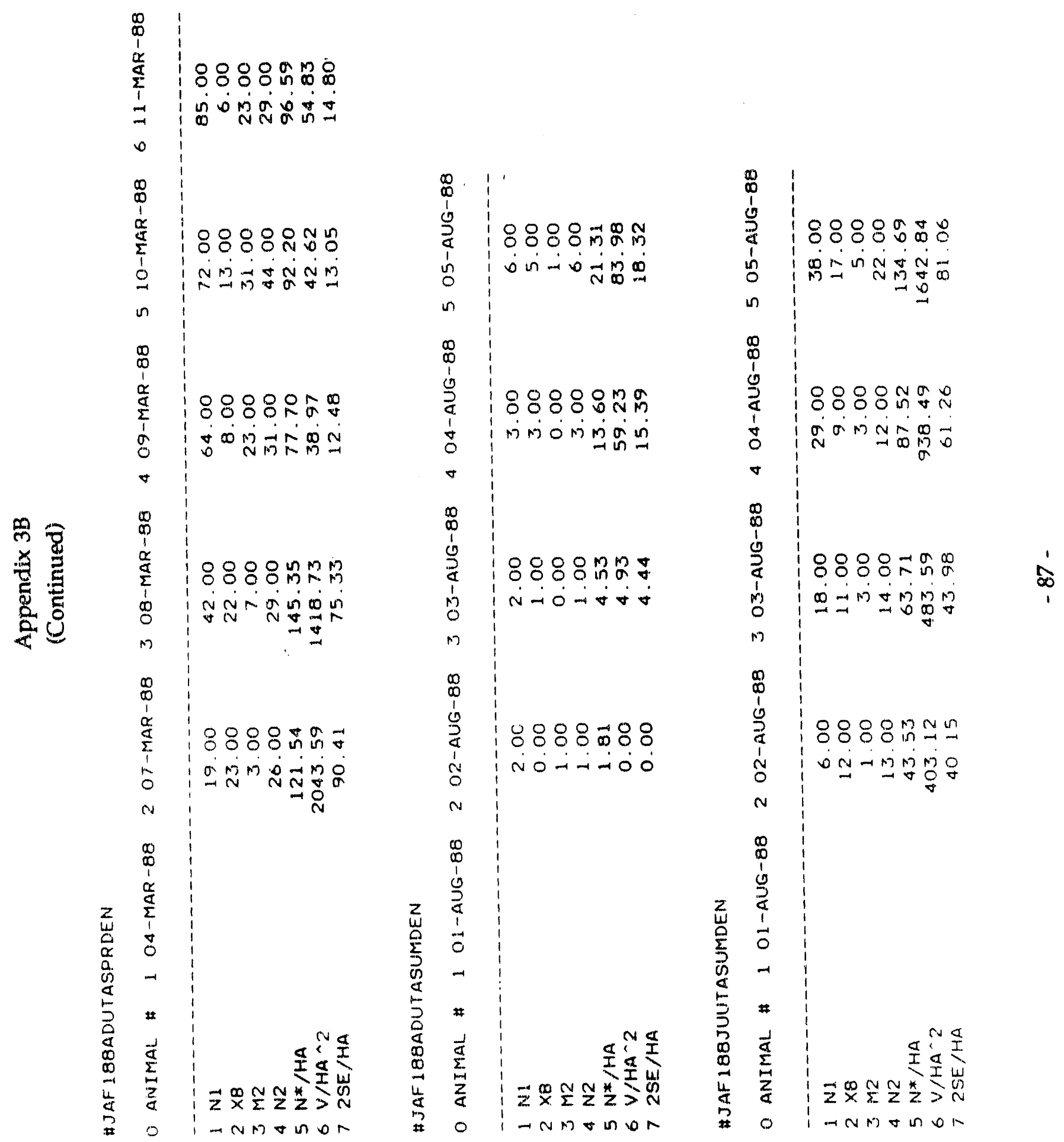



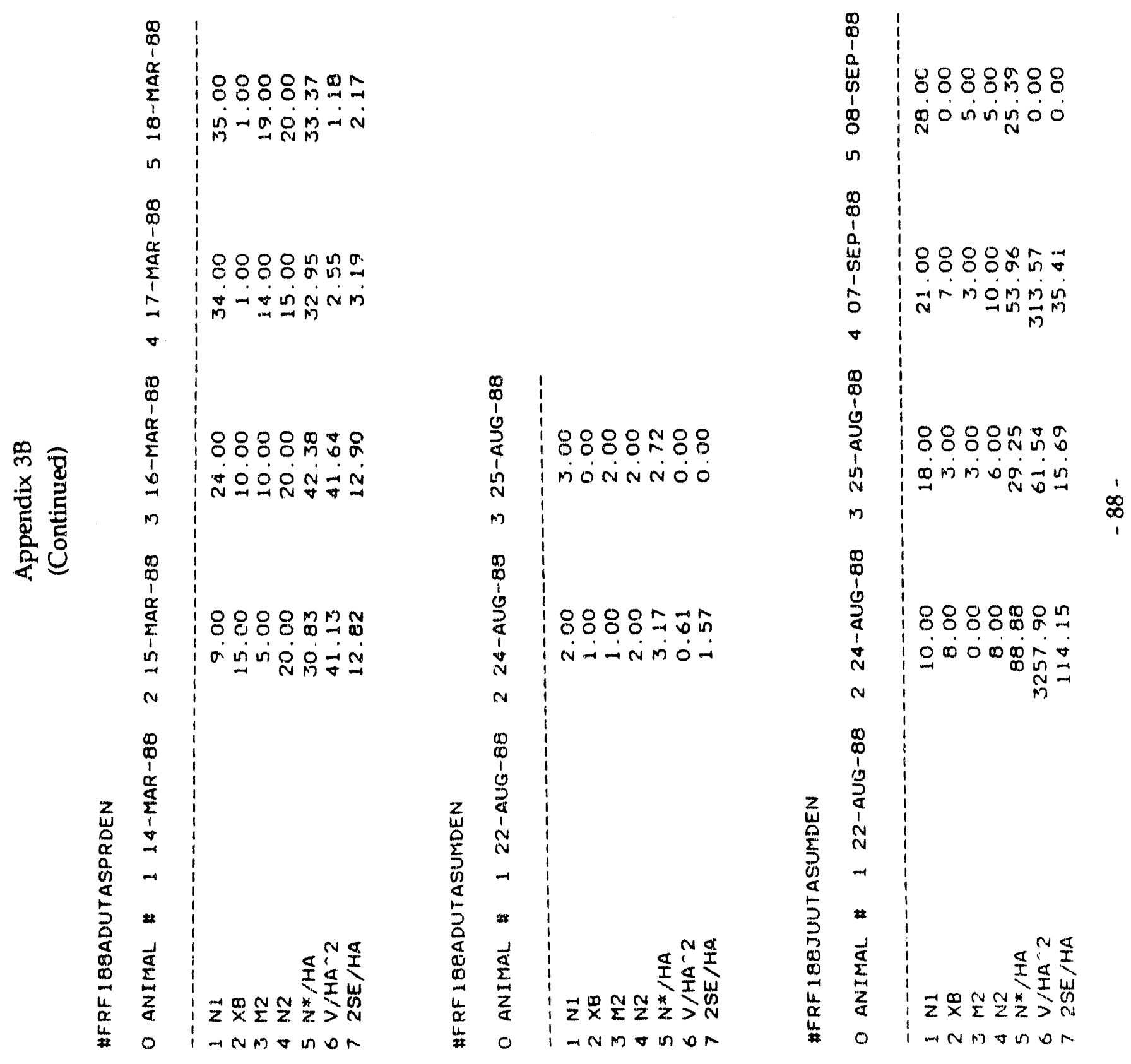

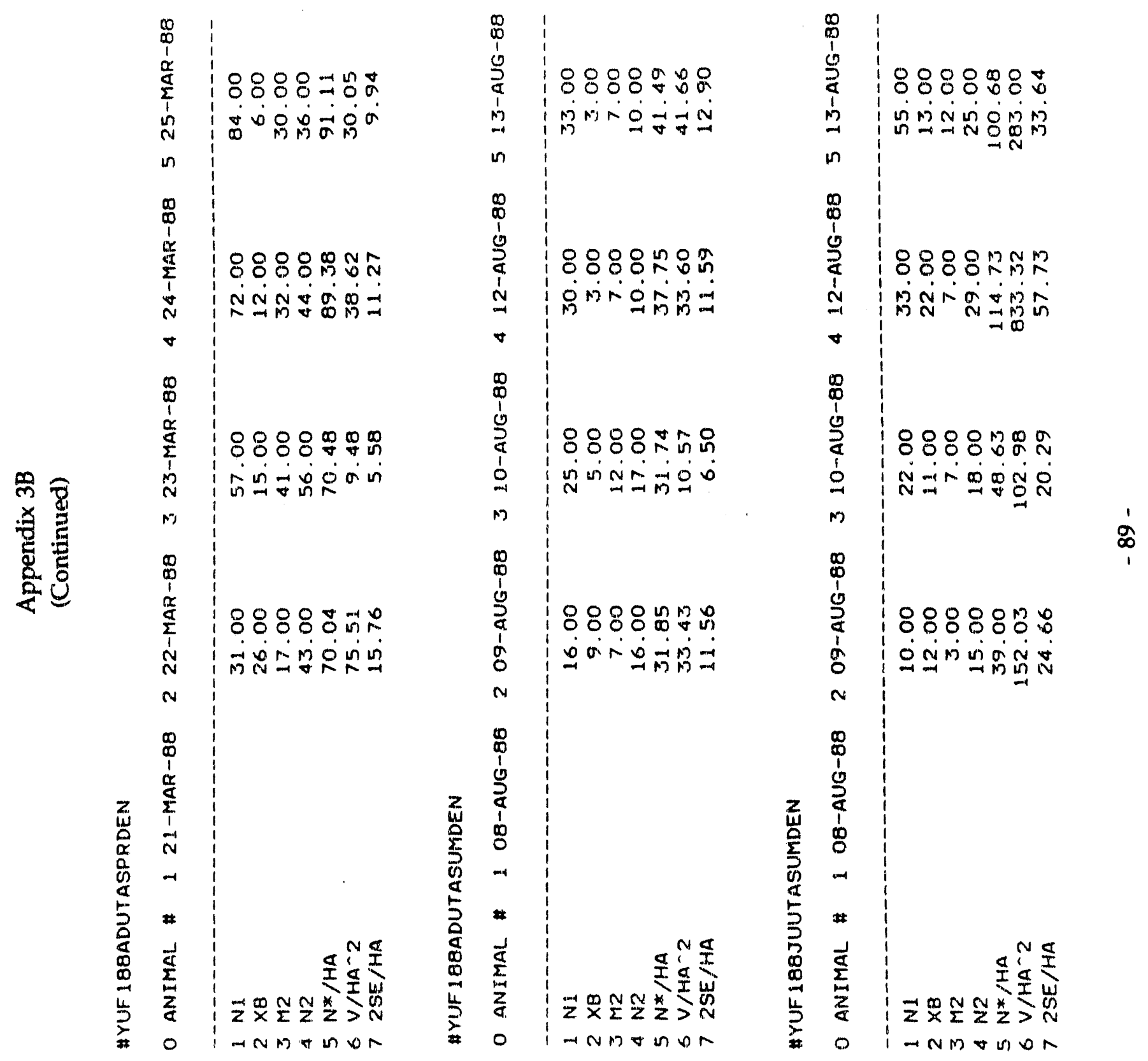

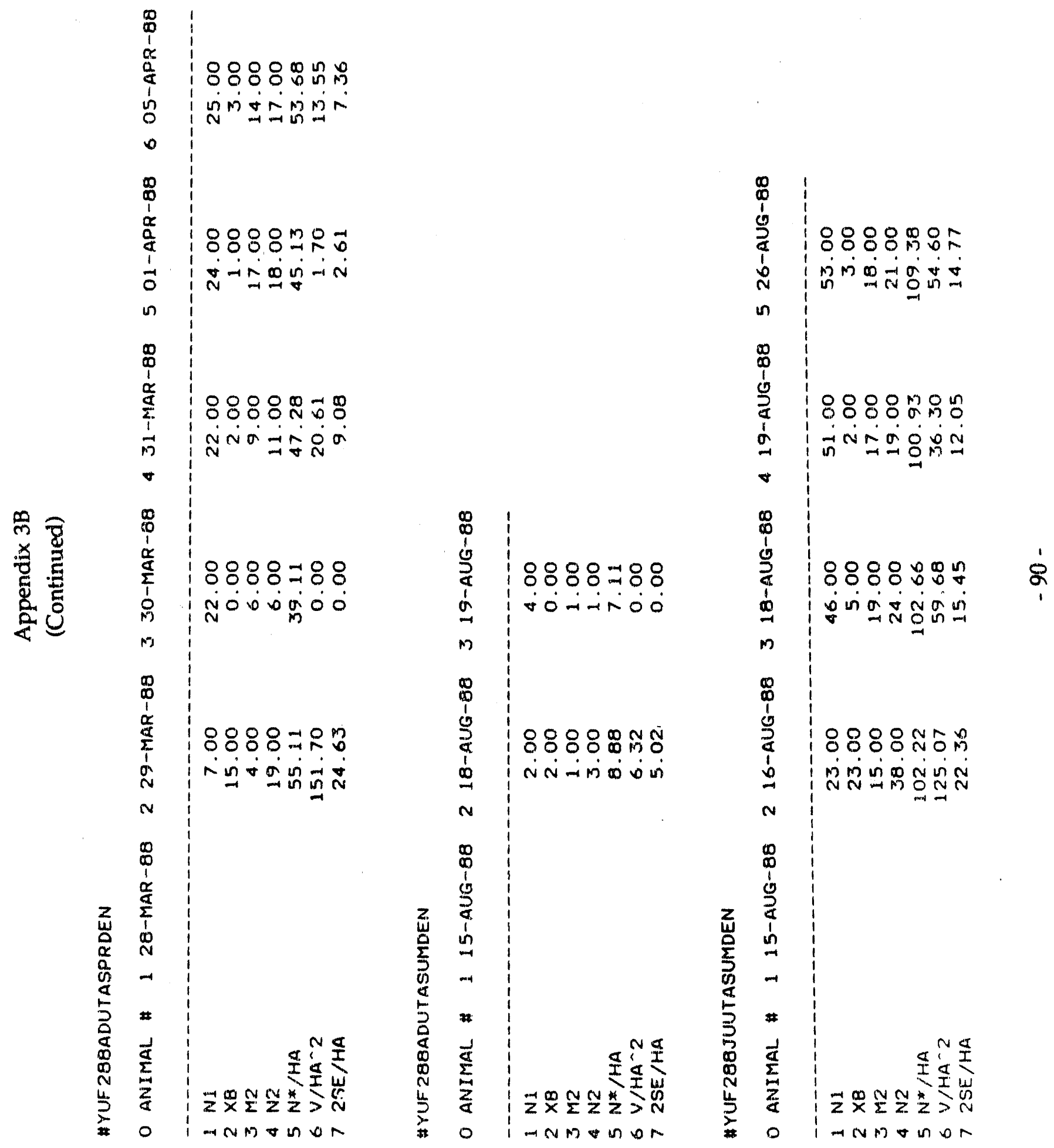


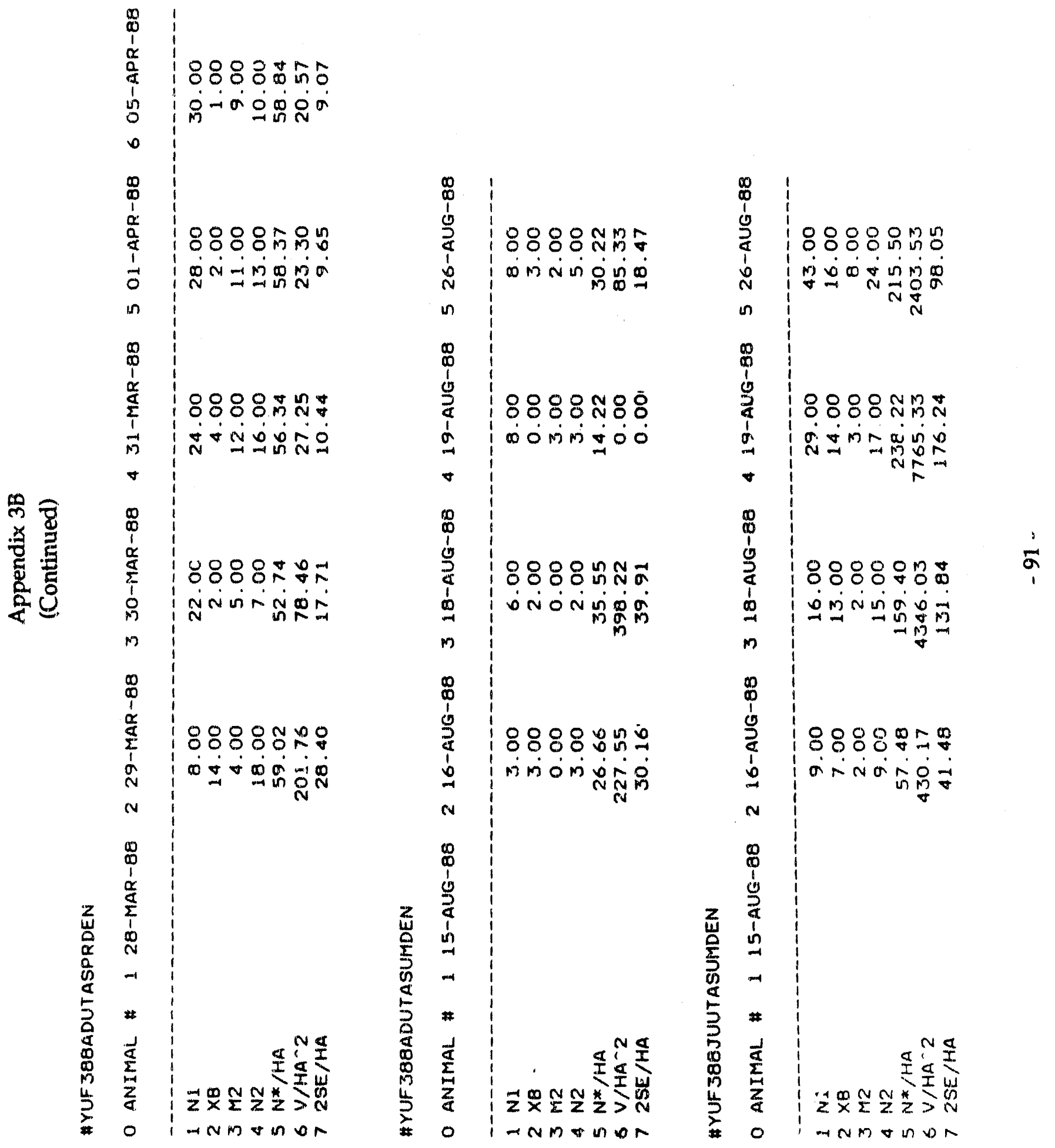



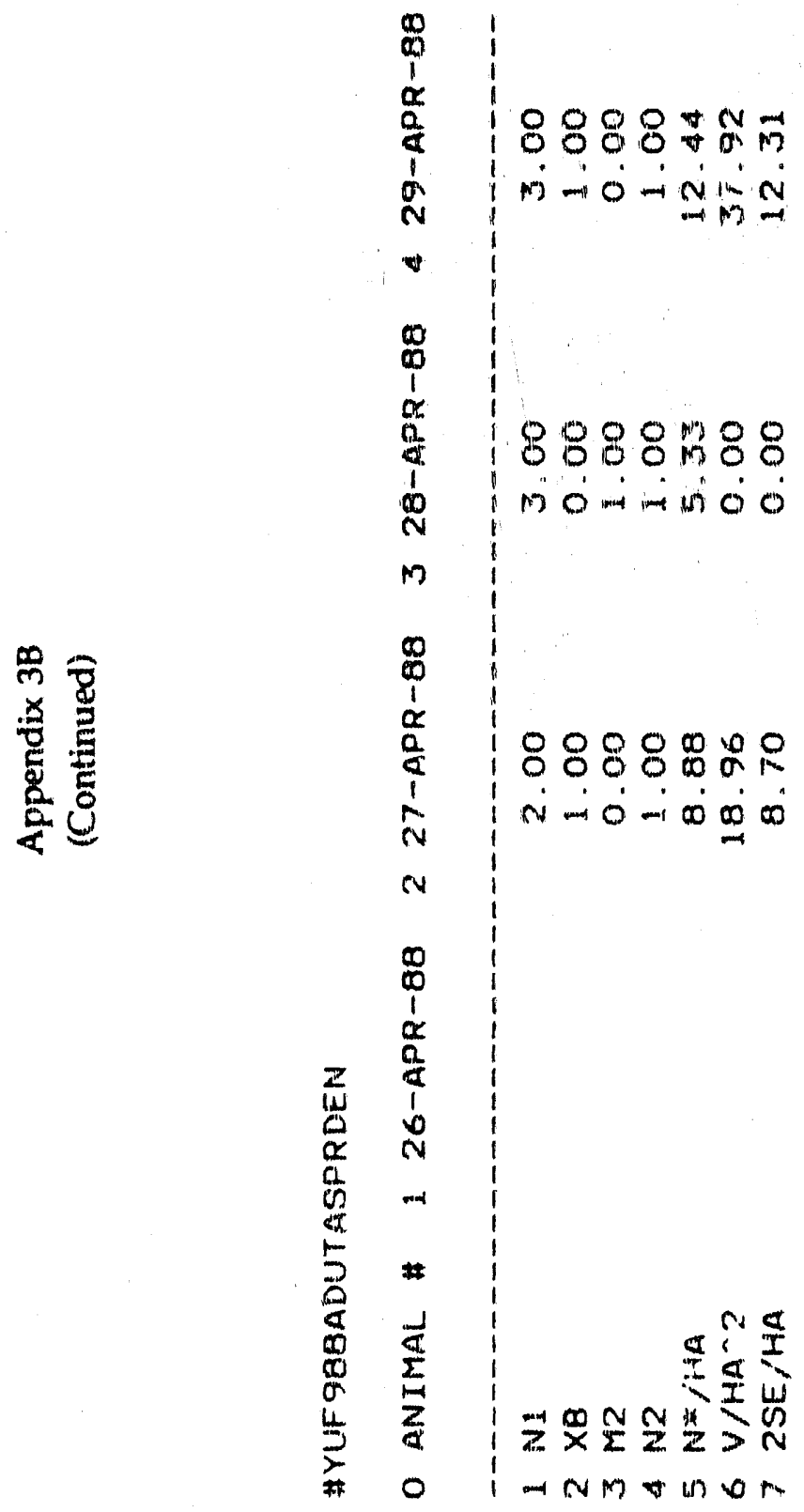

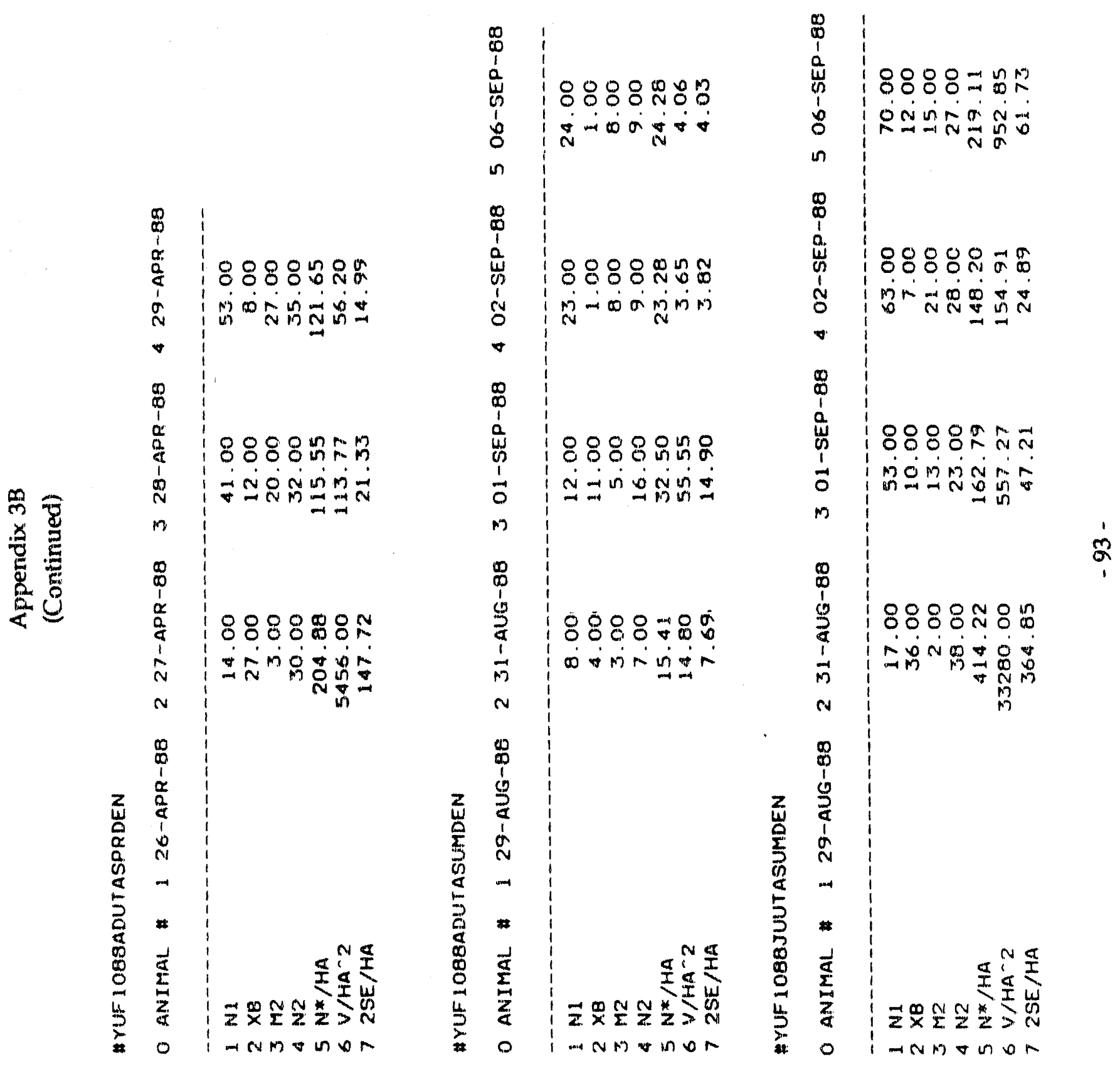

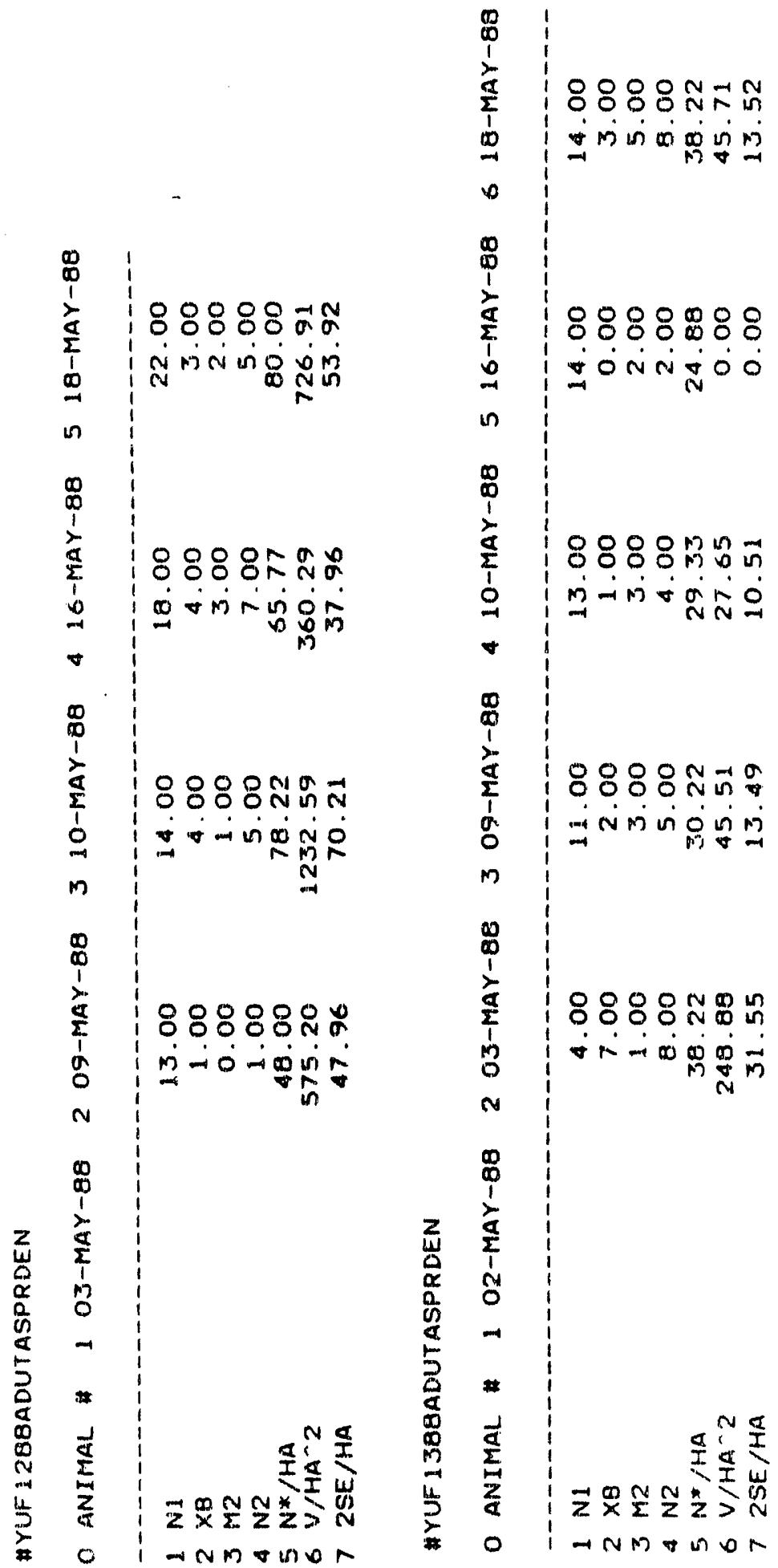

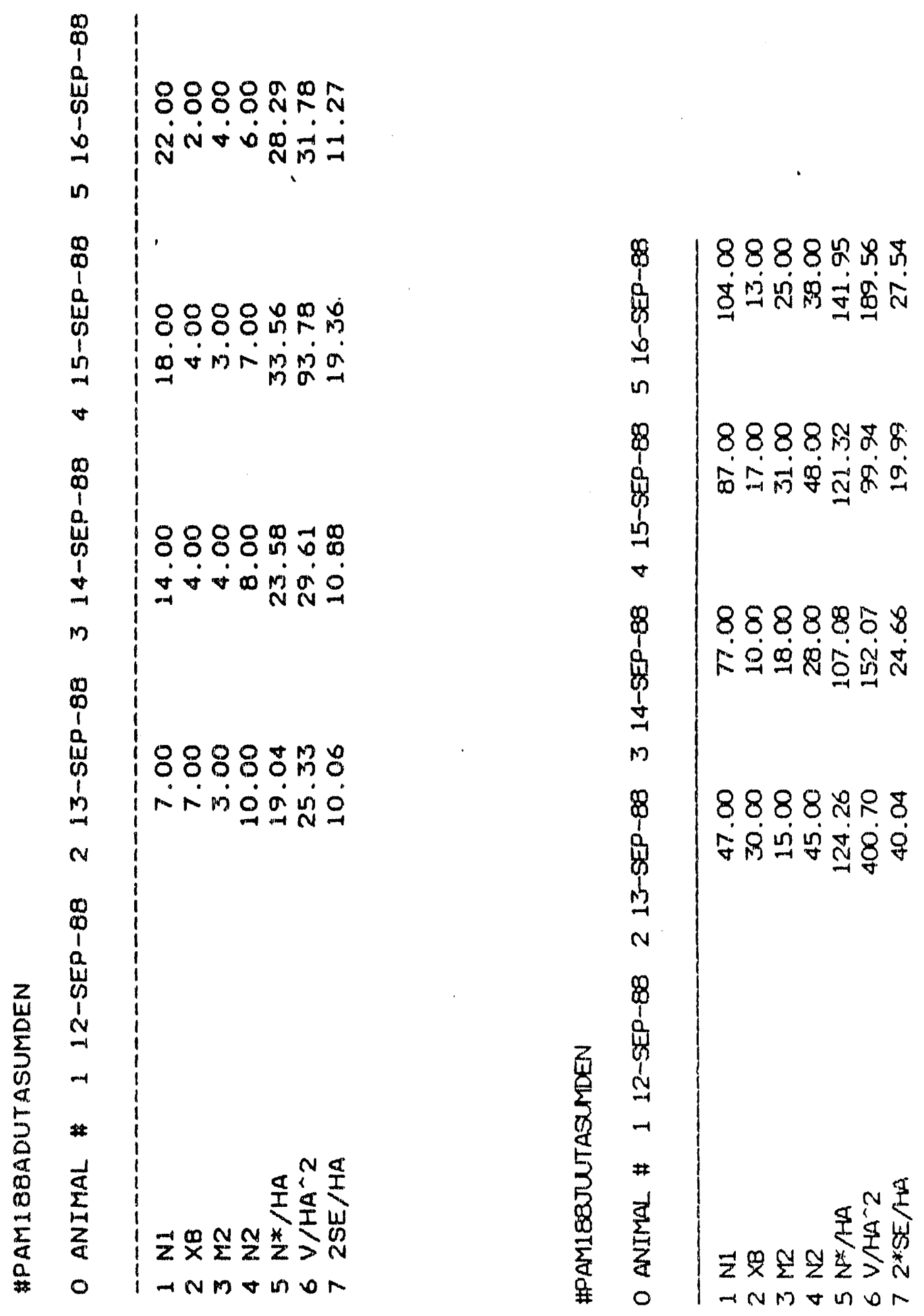
$\underline{\underline{\underline{\underline{\underline{\underline{z}}}}}}$ 


\title{
SECTION 4 \\ STATUS OF SMALL MAMMALS ON THE NTS IN 1988
}

\author{
by \\ M. B. Saethre and P. A. Medica
}

\section{INTRODUCTION}

Rodents are the most abundant mammals on the Nevada Test Site (NTS), and are common to all of the various habitats present on the NTS. Extensive studies on small mammal distribution, seasonal and daily activity patterns, home ranges and specific habitat preferences were undertaken by Brigham Young University (BYU) over a five year period from 1959 to 1965 (Allred et al. 1963; Jorgensen and Hayward 1965). A further review of the history of mammal sampling on the NTS is found in Hunter and Medica (1989).

There are three species of lagomorphs (rabbits and hares) and over twenty species of rodents present on the NTS. Several species of rodents are found throughout most of the biotic communities on the NTS. The majority of these species have patchy distributions at low densities, occupying specific plant communities and elevations, as well as the specific biotic communities characteristic of Mojave Desert and Great Basin Desert, which merge within the NTS, providing a unique ecotonal habitat. Two species of rodents found on NTS, Perognathus and Dipodomys, may be used as indicator species of different biotic communities Jorgensen and Hayward 1965).

The sampling of small mammals by the Basic Environmental Compliance and Monitoring Program (BECAMP) in 1988 consisted of resurveys of natural populations in three major valleys, Jackass Flats (JAF001), Frenchman Flat (FRF001), and Yucca Flat (YUF001), and initial surveys on two mesas, Pahute Mesa (PAM001) and Rainier Mesa (RAM001). Resident mammals on the above monitoring plots were sampled to provide baseline data on species composition, estimated densities of the more common species, sex ratios, age distribution, and biomass, as well as to document stability of rodent populations over time in areas undisturbed by NTS activities.

In addition to the baseline monitoring plots, various types of disturbances and their impact on small mammal populations were studied on subsidiary plots. The following disturbed areas were investigated: an area denuded by gophers in Mercury Valley (MER002); two sites where fires denuded the study area, one in Mid Valley (MID002) and one in Redrock Valley (REDO01); a fenced study plot (ROV008) previously exposed to a cesium-137 source ( ${ }^{37} \mathrm{Cs}$ ), and control plot (ROV007) in Rock Valley; two blast areas where aboveground nuclear tests had been conducted on towers (YUF009 and YUF013) and an adjacent waste consolidation 
site (YUF011) from which all radioactive waste and soil had recently been removed; and the area to the northeast of project Sedan in Yucca Flat (YUF016 and YUF017) which had been affected by the blast and throw-out from the 1962 underground nuclear test. Near each of the disturbed study plots, a control area was selected and sampled to document the resident species and population sizes in areas which were representative of undisturbed habitat. Locations for all of the plots sampled for mammals in 1988 are shown in Figure 4.1. Plot locations in Nevada State Grid Coordinates are listed in Appendix 3A of Section 3, "Status of Reptiles in 1988."

\section{METHODS}

\section{SMALL MAMMAL SAMPLING TECHNIQUES}

\section{Baseline Monttoring Plots}

Small mammals were trapped on the three existing BECAMP monitoring plots in 1988: JAF001, April 12-14 and August 11; FRF001, April 12-14 and August 5; and YUF001, April 2628 and August 11. These three sites had been previously trapped during the summer of 1987 and results were reported in Hunter and Medica (1989). Two new baseline monitoring sites were established and sampled in 1988: PAM001, June 24, 28, 29 and August 19; and RAM001, August 2-4. Plots at JAF001, FRF001, YUF001, and PAM001 were sampled in August to determine summer species composition (Appendix 4A).

The sampling technique consisted of three consecutive trap nights, or as close to consecutive as possible. Small nocturnal mammals were captured in Sherman live traps measuring $8 \times 9$ $\times 30$ centimeters which were set to capture animals which weighed approximately 5 grams (the average weight of a juvenile Perognathus longimembris). All mammal study plots on the baseline sites consisted of $12 \times 12$ staked grids (144 stations) with 15 meters between stakes. A total of 288 traps were placed on each study site ( 2 per station). Two traps were used to provide more opportunities for animals to be captured during the trapping period of only three days. Traps were baited in the early evening (1630+ hours) with a mixture of rolled oats and birdseed and checked shortly after sunrise the following day. Traps were oriented north-south with the opening faced away from any wind. A metal (half-cylinder) trap cover was placed over each individual trap to shade traps and prevent hyperthermia from direct sunlight.

Each rodent was permanently marked: Kangaroo rats (Dipodomys spp.) were given individually numbered ear tags in the left ear and, on long-term. study plots, a toe was clipped to indicate a numbered animal in case the ear tag was lost. All other rodents were toe clipped with no more than one toe amputated per foot. Species, capture status (new or recapture), animal number, sex, seproductive condition, and grid location were recorded on field data sheets. The toe clipping formula and field data sheets are illustrated in the 1987 annual report (Hunter and Medica 1989, Figures 14 and 15). Each animal was weighed to the 


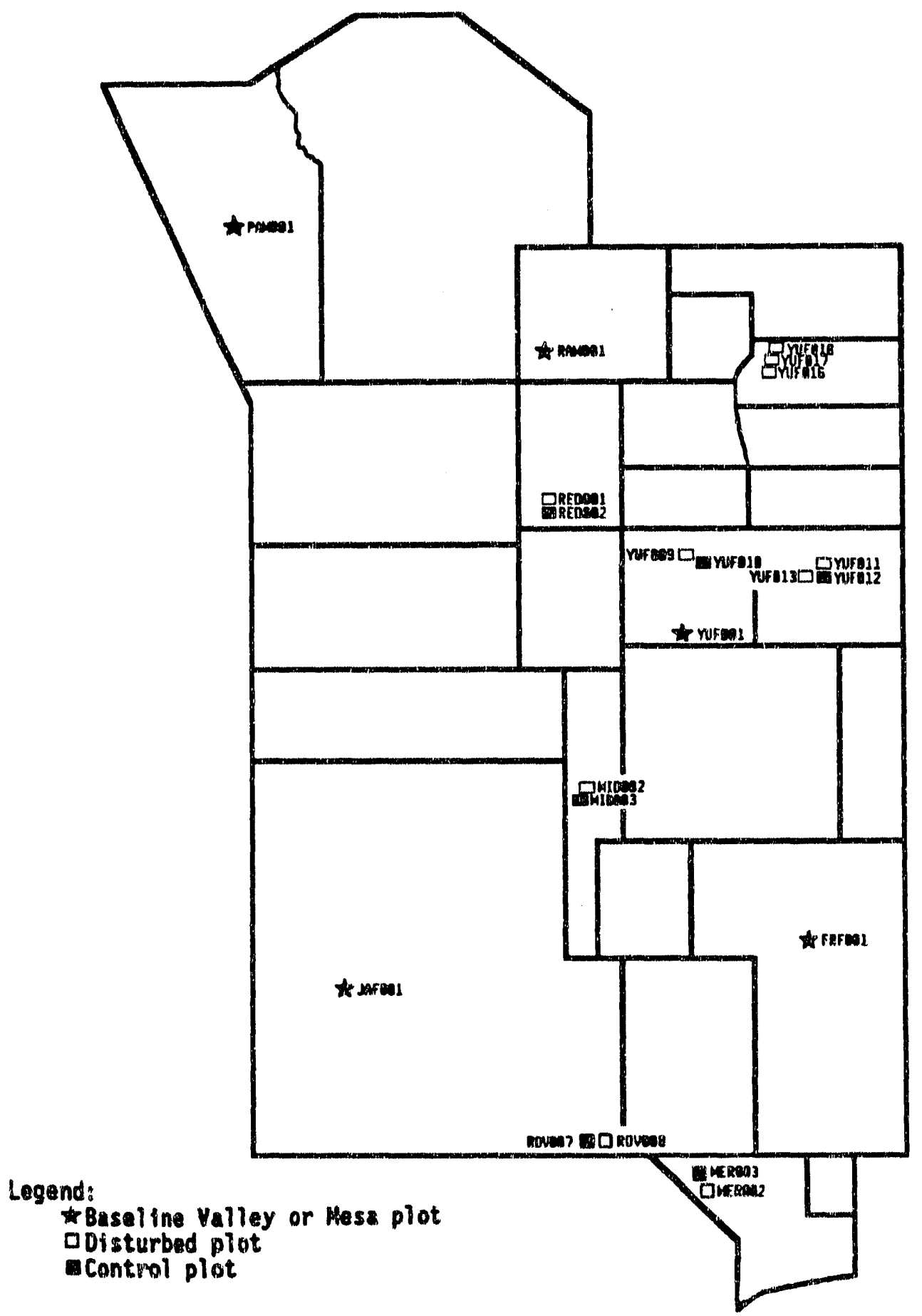

Figure 4.1 Locations of 1988 BECAMP small mammal study plots. 
nearest gram using a spring scale and released at the point of capture. Mean weights were analyzed using ANOVA or Student's t-test where appropriate.

\section{Subsidiary Plots}

Procedures on the subsidiary plots did not differ significantly from those on the baseline monitoring sites. However, plot size was smaller and a disturbed and control site were sampled at the same time. Each subsidiary plot site was staked and gridded at 15-meter intervals with the size of grid adjusted to the size of habitat available to be sampled. In general, an $8 \times 8$ grid was used. The two burned areas were long and narrow, therefore a 15 x 5 grid in Mid Valley (MID002) and a $14 \times 5$ grid in Redrock Valley (RED001) were used on both the controls and disturbed areas. The T3 (YUF013), 3B consolidation site (YUF011), and control (YUF012) were also $14 \times 5$ grids.

\section{Trap Density Experiment}

Immediately after completion of trapping for small, nocturnal mammals on the baseline plots in Jackass Flats (JAF001) and Frenchman Flat (FRF001), traps were rearranged for a trap density experiment to determine whether or not the present number of traps per hectare at a distance of 15 meters between traps was adequate for sampling a population. Four smaller grids were established within the existing $12 \times 12$ grid as follows: a $10 \times 10$ grid $(0.46 \mathrm{ha})$, 100 traps placed at $7.5-\mathrm{m}$ intervals $(51 \mathrm{traps} / \mathrm{ha})$; a $7 \times 7$ grid $(0.41 \mathrm{ha}), 49$ traps placed at $10.7-\mathrm{m}$ intervals ( 69 traps/ha); a $5 \times 5$ grid ( 0.36 ha), 25 traps placed at 15-meter intervals (119 traps/ha); a $4 \times 4$ grid (0.32 ha), 16 traps placed at $18.75-\mathrm{m}$ intervals ( 219 traps $/ \mathrm{ha})$. The placement of the smaller grids was chosen at random. However, the smaller grids were placed at the same relative location within both plots. A diagram of the placement of the traps in the larger grid is shown in Figure 4.2.

The traps were set for only one night, rather than three. Locations of animals captured during the three days of trapping the $12 \times 12$ grid were mapped out on the smaller grid. These animals and locations were considered to be the number of animals which could be captured on that respective grid. The $10 \times 10$ and $4 \times 4$ grid total number of animals possible included edge animals captured at traps less than $7.5 \mathrm{~m}$ away. The number of recaptured and newly captured animals were enumerated and percent success (recaptures/number possible) was calculated for each small grid. The densities of Perognathus longimembris and Dipodomys merriami (the most common species on both plots) were calculated for the one night of trapping by dividing the total number of animals captured by the smaller plot size in hectares, and were compared to the density calculated during the three nights of trapping. It was hypothesized that as trap density increased, animal density would increase up to a certain point and then level off. It was also thought that as the number of traps increased, the percent trap success would decrease. 
$4 \times 4(18.75 \mathrm{~m})$

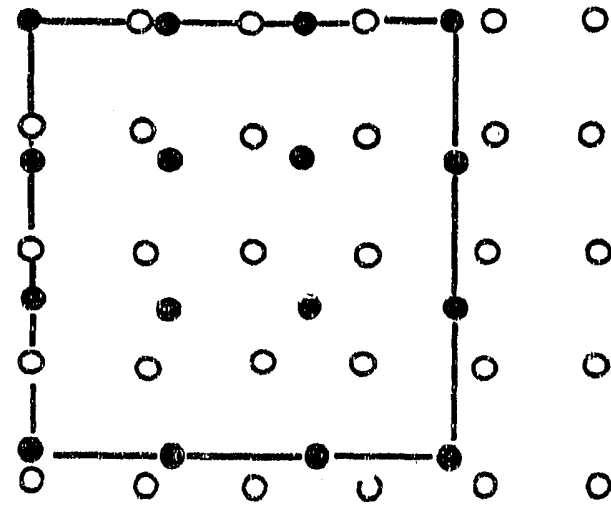

$\begin{array}{llllll}0 & 0 & 0 & 0 & 0 & 0\end{array}$

0000000

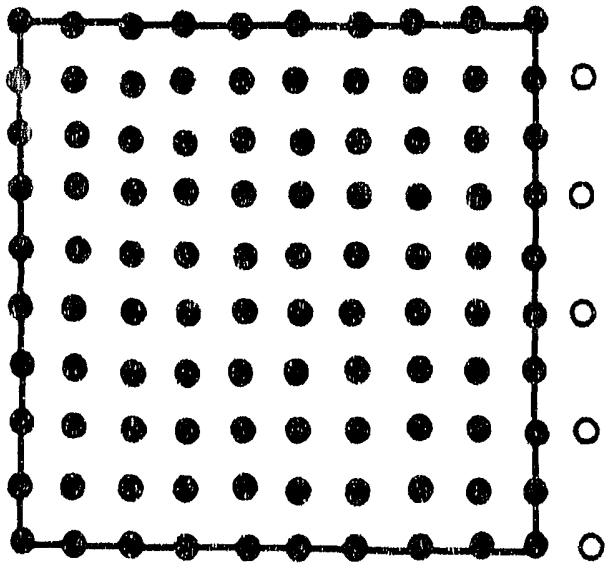

$10 \times 10(7.5 \mathrm{~m})$
$7 \times 7(10.7 \mathrm{~m}) \quad N$

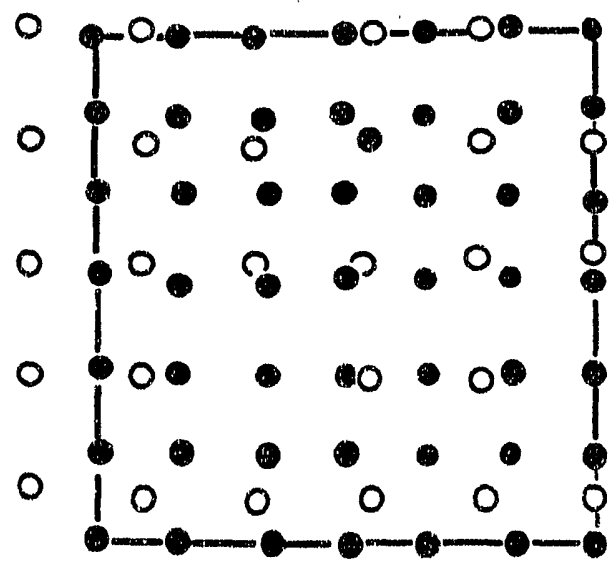

$\begin{array}{llllll}0 & 0 & 0 & 0 & 0 & 0\end{array}$

$\begin{array}{llllll}0 & 0 & 0 & 0 & 0 & 0\end{array}$

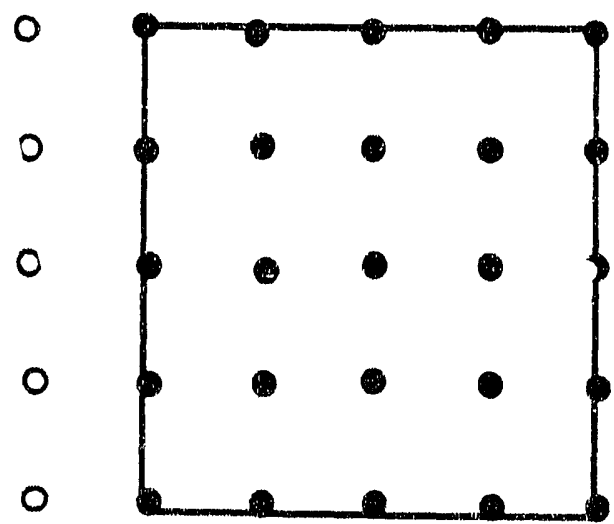

$5 \times 5(15.0 \mathrm{~m})$

- Location of trap on original $12 \times 12$ grid.

Location of trap on smaller grid.

Figure 4.2 Placement of the $4 \times 4,5 \times 5,7 \times 7$, and $10 \times 10$ grids inside of the larger, $12 \times 12$, grids on the Jackass Flats and Frenchman Flat baseline monitoring plots. 


\section{Density Estimation}

The first night of the three nights of trapping was considered a preliminary trap night. The population of small mammals was estimated by using the following capture/recapture formula (Seber 1982:138) and data from the second and third nights of trapping:

$$
N *=\frac{\left(n_{1}+1\right)\left(n_{2}+1\right)}{\left(m_{2}+1\right)}-1
$$

$\mathrm{N}^{*}=$ Population estimate, number per plot.

$\mathrm{n}_{1}=$ Total individuals marked before the present trap night.

$\mathrm{n}_{2}=$ Total individu ils captured during the present trap night.

$\mathrm{m}_{2}=$ Number in $\mathrm{n}_{2}$ which were recaptured (marked in a previous sample).

The standard error (SE) of the population estimate was calculated using the following formula (Seber 1982:138):

$$
S E=\sqrt{\frac{\left(n_{1}+1\right)\left(n_{2}+1\right)\left(n_{1}-m_{2}\right)\left(n_{2}-m_{2}\right)}{\left(m_{2}+1\right)^{2}\left(m_{2}+2\right)}}
$$

Calculations using these two formulas give an estimate of population in number of animals per plot plus or minus the standard error. To estimate the density in number of animals per hectare \pm the standard error, $N^{*}$ and SE were divided by the plot size in hectares, which included a $7.5-\mathrm{m}$ perimeter (half of the distance between trap stations) to account for effective trapping area. To get a suitable estimation of density and standard error from equations 1 and $2, \mathrm{~m}_{2}$ (number of recaptures) should be greater than 7 (Seber, 1982). Therefore, densities for rare (i.e. infrequently captured) species were not calculated. Equation 1 assumes a closed population with a constant number of animals during the capture-recapture time period. For trapping times of short duration, immigration, emigration, reproduction and death may be ignored and these equations should be adequate to estimate population densities. A standard error of zero arose when all marked animals were recaptured on the last day $\left(n_{1}-m_{2}\right)$ or no new animals were captured on the last day and, therefore, all of the captures were recaptures $\left(n_{2}-m_{2}\right)$.

\section{Dlurnal Rodents (Ground Squirrels)}

Trapping for ground squirrels was conducted during August 1988 for one day on the baseline study plots in Jackass Flats and Yucca Flat to determine size, age distribution, and activity pattern of the resident population. Squirrel live traps ('Tomahawk' live trap \#102, $13 \times 13 \times 40$ centimeters, set to close at a minimum weight of 30 grams) were placed at every-other grid stake on each of the 12 lines of the $12 \times 12$ trapping grid (72 total traps). Traps were opened in early morning ( 0800 hours) and were left open throughout the day 
until late afternoon (1600 hours). The traps were baited with rolled oats and bird seed and checked at two-hour intervals. Captured animals were toe-clipped, sex and age determined, weighed, and released at the capture location. Shades of $30.5 \times 30.5 \times 0.5$-centimeter masonite were placed over the traps and continuously repositioned throughout the day to shade any trapped animals.

\section{Rabblt and Hare Sampling (Transect Lines)}

These censuses on the NTS were performed concurrently with line transects for lizard sampling. The design of the lizard transect lines is illustrated in Hunter and Medica (1989, Figure 2) and in Figure 3.2 of this report. Transect lines were simultaneously walked by three observers, 7.5 meters apart, in late spring and early summer of 1988 . On the baseline sites, observers walked the 500-meter length of each of the five transect lines (total of $2500 \mathrm{~m}$ ). 'Transects on subsidiary plots consisted generally of walking around the perimeter of the area in a square. When a rabbit or hare was observed, the flushing distance and direction when first observed were estimated and recorded on a $3 \times 5$-inch card or the bottom of the lizard transect data sheet.

To obtain density (D) in number per hectare, the following formula was used (Whitford, 1973):

$$
D=\left(\frac{\mathrm{N}}{2 \mathrm{rL}}\right) \times\left(10,000 \mathrm{~m}^{2} / \mathrm{ha}\right)
$$

$\mathrm{L}=$ the total distance walked in the transect in meters

$\mathrm{N}=$ the number of flushes

$r=$ the mean flushing distance estimated in meters

The estimated densities of a species were averaged for all of the days sampled (usually five days) and the standard error calculated for this average. Estimated densities and species observed were compared between different habitats which occur on the NTS.

\section{RESULTS}

Species' names appear in results tables as the abbreviations shown in Table 4.1. Estimates of rodent density (number per hectare \pm two standard errors) for all five of the baseline monitoring sites appear in Table 4.2. Descriptions of the flora of each plot are found in the sections entitled Status of Perennial Vegetation and Status of Desert Ephemeral Plants in this report. Percent of total captured population for each species appears in Table 4.3. 
Table 4.1 Abbreviations for scientific names of small mammals captured on the NTS and the common names.

\begin{tabular}{llll} 
& \multicolumn{1}{c}{ Scientific Name } & \multicolumn{1}{c}{ Common Name } \\
\cline { 2 - 3 } AMM LEU & Ammospermophilus leucurus & & White-tailed antelope squirrel \\
DIP DES & Dipodomys deserti & & Desert kangaroo rat \\
DIP MER & Dipodomys merriami & & Merriam's kangaroo rat \\
DIP MIC & Dipodomys microps & Great Basin kangaroo rat \\
DIP ORD & Dipodomys ordii & Ord's kangaroo rat \\
LEP CAL & Lepus californicus & Black-tailed jackrabbit \\
MIC MEG & Microdipodops megacephalus & Dark kangaroo mouse \\
MUS FRE & Mustela frenata & Long-tailed weasel \\
NEO LEP & Neotoma lepida & Desert woodrat \\
ONY TOR & Onychomys torridus & Southern grasshopper mouse \\
PER FOR & Perognathus formosus & Long-tailed pocket mouse \\
PER LON & Perognathus longimembris & Little pocket mouse \\
PER PAR & Perognathus parvus & Great Basin pocket \\
PER CRI & Peromyscus crinitus & Canyon mouse \\
PER MAN & Peromyscus maniculatus & Deer mouse \\
PER SPP & Peromyscus spp. & \\
PER TRU & Peromyscus truei & Pinon mouse \\
REI MEG & Reithrodontomys megalotis & Western harvest mouse \\
SPE TER & Spermophilus tereticaudus & Round-tailed grouris squirrel \\
SYL AUD & Sylvilagus audubonii & Desert cottontail \\
SYL NUT & Sylvilagus nuttallii & Nuttall's cottontail \\
TAM DOR & Tamias (=Eutamias) dorsalis & Cliff chipmunk \\
THO UMB & Thomomys umbrinus & Southern pocket gopher \\
& &
\end{tabular}

* Peromyscus species not distinguished.

\section{BASELINE MONITORING PLOTS}

The rnost common rodents, Dipodomys merriami and Perognathus longimembris, accounted for $99.0 \%$ and $97.6 \%$ of the captured populations of individual animals in Jackass Flats and Frenchman Flat, respectively (Table 4.3). In Yucca Flat, $92.7 \%$ of the captured population consisted of $D$. merriami, $D$. microps, and $P$. longimembris. The percent composition of these three sites in 1987 (Hunter and Medica 1989) were comparable $(97.7 \%, 93.4 \%$, and $91.6 \%$ respectively) although they were trapped later in the summer of 1987. On the Pahute Mesa plot, $86.7 \%$ of the captured population was Perognathus parous and Peromyscus species. On the Rainier Mesa plot, no one species accounted for more than $50 \%$ of the captured individual animals, with $66.6 \%$ of the captured population consisting of Peromyscus spp. and Tamias dorsalis. Differences in species composition on the 5 baseline plots can be accounted for by habitat preference of the individual species (e.g., elevation, shrub cover, soil composition, moisture, temperature and season). 


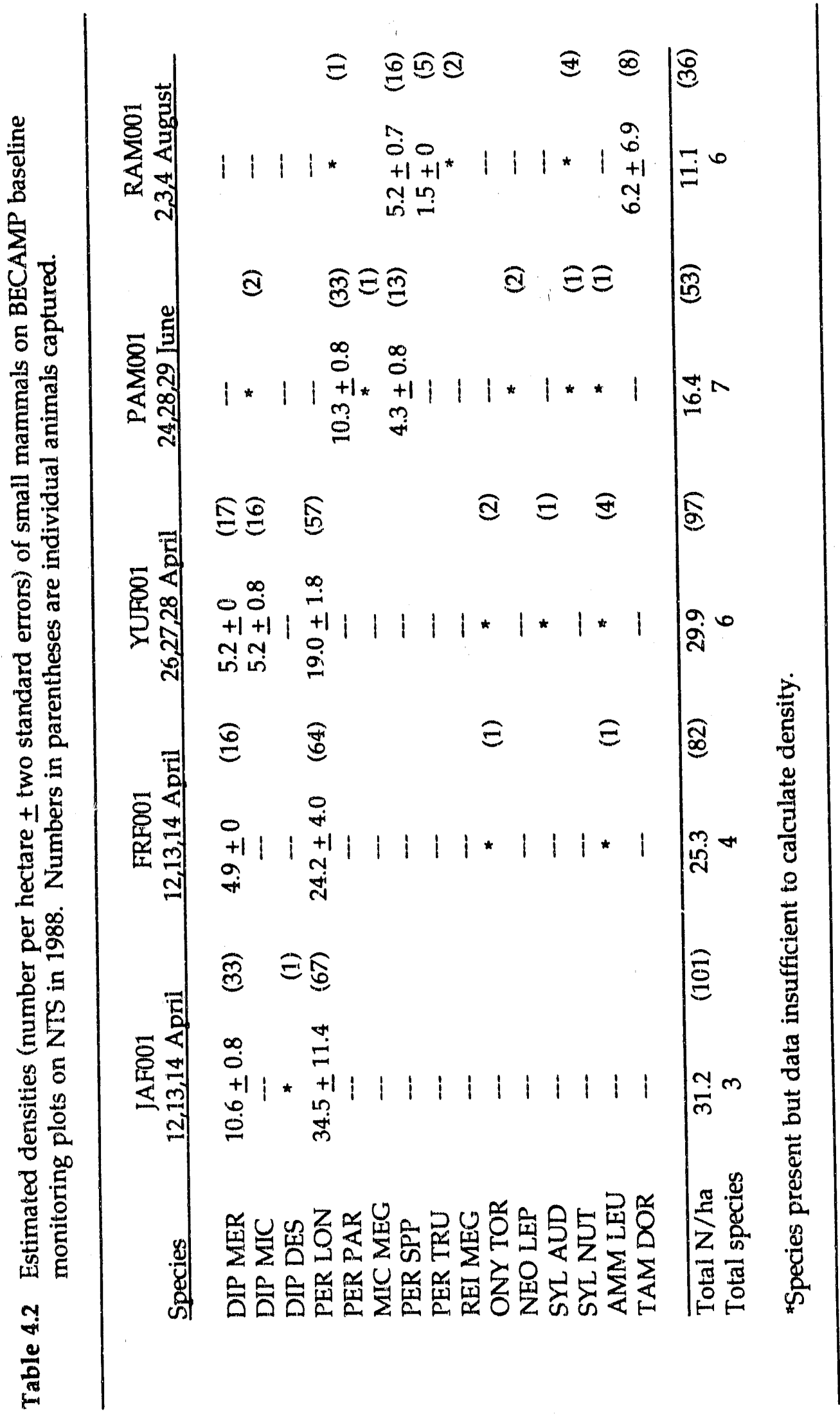




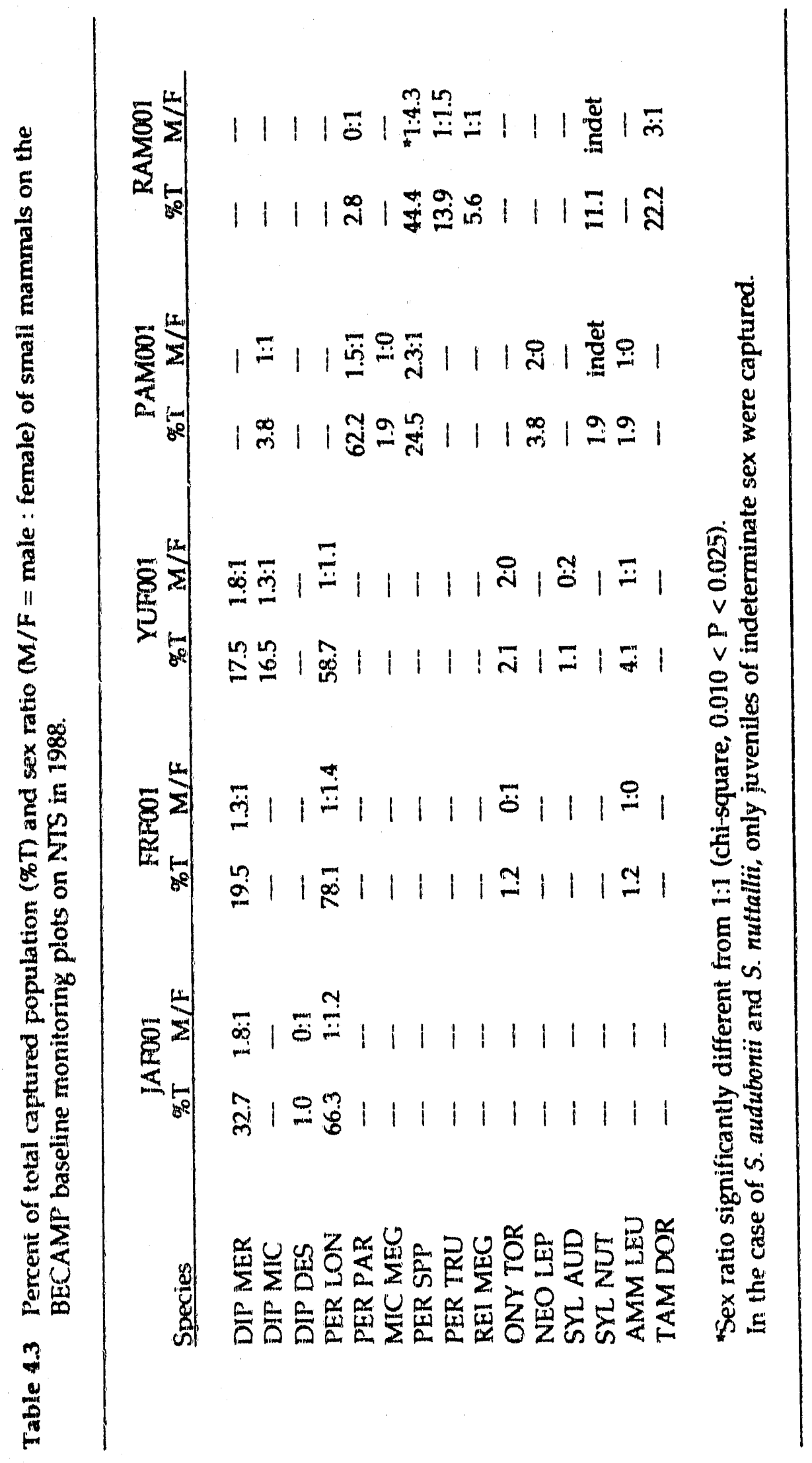


Species captured in 1987 but not in 1988 on the Jackass Flats baseline monitoring site were the diurnal squirrels Spermophilus tereticaudus and Ammospermophilus leucurus. However, while no A. leucurus were trapped during the 1988 nocturnal mammal trapping in Jackass Flats, they were captured during daytime squirrel trapping in August. In 1987 S. tereticaudus was also captured on the Frenchman Flat baseline site but not in 1988.

Peromyscus maniculatus was not captured during mammal trapping on the Yucca Flat site in 1988 but was present in 1987. One Reithrodontomys megalotis and a juvenile Sylvilagus audubonii were captured in August of 1988 but not in April 1988 nor in 1987. Neotoma lepida and A. leucurus were not captured during summer trapping in August on the Pahute Mesa plot. A complete list of species captured during spring and summer on the BECAMP baseline monitoring sites is shown in Appendix 4A.

A sum of 369 individual animals from 15 different species were marked and released during spring trapping for a total of 652 captures on the baseline monitoring sites. Trapping on each plot involved 864 trap nights (288 traps $\times 3$ nights), with 101 different animals captured a total of 159 times on JAF001, 146 captures of 82 animals on FRFO01, 192 captures of 97 animals on YUF001, 98 captures of 53 animals on PAM001, and 57 captures of 36 animals on RAM001. Percent trap success on the above plots (total captures divided by trap nights) were 18.4\% (JAF001), 16.9\% (FRF001), 22.2\% (YUF001), 11.3\% (PAM001), and 6.6\% (RAM001). Overall trap success was $15.1 \%$.

Individual animals were captured from an average low of 1.6 times on RAM001 to a high of 2.0 on YUF001, which was the same for Yucca Flat in 1987 (Hunter and Medica 1989). Average capture frequency in 1988 for JAF001 was 1.6 (1.6 also in 1987) and for FRF001, 1.8 (1.9 in 1987). Differences in trap success and average capture frequency among plots in 1988 are probably due to the differences in vegetation type and percent cover of vegetation between each plot and the difference in horne ranges of the different species.

In general, the ratio of males to females for the most common species (Table 4.3) did not differ significantly from a $1: 1$ ratio (chi-square analysis, $p>0.05)$. However, $81.3 \%(1: 4.3, n=$ 16) of the Peromyscus spp. captured on the Rainier Mesa plot were female. From the combined plots of JAF001, FRF001 and YUF001, there was a significant difference from the 1:1 Dipodomys merriami sex ratio, with females comprising only $37.9 \%(1: 1.6, n=66)$ of the $D$. merriami captured. On the other hand, Perognathus longimembris did not differ significantly from a $1: 1$ ratio with females comprising $56.4 \%(1: 1.3, n=188)$ of the $P$. longimembris captured.

Spring mean weights for the most abundant species on Jackass Flats, Frenchman Flat, and Yucca Flat are shown in Table 4.4. The mean weight of male Dipodomys merriami captured on the Yucca Flat monitoring plot was greater than that of male D. merriami captured on the Frenchman Flat and Jackass Flats monitoring sites, but not with any significance. The mean 
Table 4.4 Adult spring mean weights (grams \pm 2 SE) by sex of common rodent species on three BECAMP baseline monitoring plots in 1988.

\begin{tabular}{|c|c|c|c|c|c|c|c|}
\hline \multirow[b]{2}{*}{ Species } & \multirow[b]{2}{*}{ Sex } & \multicolumn{2}{|c|}{ JAF001 } & \multicolumn{2}{|c|}{ FRF001 } & \multicolumn{2}{|c|}{ YUF001 } \\
\hline & & $N$ & Weight & $N$ & Weight & $N$ & Weight \\
\hline PER LON & $\begin{array}{l}M \\
F\end{array}$ & $\begin{array}{l}31 \\
36\end{array}$ & $\begin{array}{l}7.3 \pm 0.3 \\
7.4 \pm 0.3\end{array}$ & $\begin{array}{l}25 \\
39\end{array}$ & $\begin{array}{l}7.8 \pm 0.3 \\
7.5 \pm 0.3\end{array}$ & $\begin{array}{l}27 \\
30\end{array}$ & $\begin{array}{l}8.3 \pm 0.3 \\
8.0 \pm 0.2\end{array}$ \\
\hline DIP MER & $\begin{array}{l}M \\
F\end{array}$ & $\begin{array}{l}21 \\
12\end{array}$ & $\begin{array}{l}40.9 \pm 1.3 \\
41.6 \pm 2.3\end{array}$ & $\begin{array}{l}9 \\
7\end{array}$ & $\begin{array}{l}41.6 \pm 1.0 \\
39.7 \pm 2.9\end{array}$ & $\begin{array}{c}11 \\
6\end{array}$ & $\begin{array}{l}42.5 \pm 0.9 \\
39.6 \pm 2.0\end{array}$ \\
\hline DIP MIC & $\begin{array}{l}\mathrm{M} \\
\mathrm{F}\end{array}$ & $\begin{array}{l}0 \\
0\end{array}$ & - & $\begin{array}{l}0 \\
0\end{array}$ & - & $\begin{array}{l}9 \\
7\end{array}$ & $\begin{array}{l}63.2 \pm 4.1 \\
58.8 \pm 5.2\end{array}$ \\
\hline
\end{tabular}

weight of $D$. merriami males did not differ significantly between Frenchman Flat and Jackass Flats, nor cid females between the two plots. There was no significant difference between the combined mean adult weight (male and female) for D. merriami trapped at Yucca Flat and Frenchman Flat, nor between Frenchman Flat and Jackass Flats or Yucca Flat and Jackass Flats. The mean weight of adult male $D$. merriami was significantly greater than adult female D. merriami in Yucca Flat (ANOVA, $p=0.0087$ ) but not in Frenchman Flat or Jackass Flats. This is likely due to the females in Yucca Flat being reproductively active later due to the cooler temperatures of the higher latitude and altitude - during reproduction, female weights differ the least from those of males (Kenagy 1973). Mean summer weights of Dipodomys merriami for both sexes were lower than spring mean weights on all three plots (Table 4B-1, Appendix 4B), most likely due to the addition of lower-weighted subadults.

The mean weights of Perognathus longimembris males in Yucca Flat were significantly greater than those in Frenchman Flat and Jackass Flats (ANOVA, $p<0.05$ ). The mean weights of adult female $P$. longimembris were also significantly heavier on the Yucca Flat baseline plot than on the Frenchman Flat plot $(0.001<p<0.05)$ and the Jackass Flats plot $(p<0.001)$, while mean weights of males and females captured in the same valley did not differ significantly.

\section{SUBSIDIARY MONITORING SITES - DISTURBED AND UNDISTURBED PLOTS}

\section{Disturbance Due To Fire}

Two pairs of subsidiary plots were sampled in 1988 to monitor the impact of fire on the resident population of small mammals. Each pair consisted of a plot in the burned area and one control plot in an adjacent, unburned area. Vegetation for the Mid Valley sites are characterized in the plant section of the 1987 report (Hunter and Medica 1989). Vegetation on the Redrock burn sites was not sampled until 1989. The burned area in Mid Valley was a long, narrow strip of land. The control for this site was less than 30 meters to the south of 
the burned plot. This area in Mid Valley burned in June 1986 due to a lightning strike, and was sampled 3,7 , and 8 June 1988.

The plots in the burned and undisturbed areas of Redrock Valley were also set up as long and narrow grids. A paved road separated the control from the burned area in this valley. The probable cause for the fire in Redrock Valley was listed as a lit cigarette. The fire was started and extinguished on 20 July 1988, incinerating an estimated 160 to 200 ha (400 to 500 acres). A study plot was immediately constructed to monitor the species which might have survived and if recolonization might occur. These plots were trapped on 26-28 July ( 6 days after the fire), 24-25 August (1 month after the fire) and 26-28 October ( 3 months after the fire).

\section{Mid Valley}

The estimated densities of the most commonly captured species on the burned and unburned plots in Mid Valley are given in Table 4.5. Most of the species which occurred on the control were also present on the burned plot with the exception of one adult Reithrodontomys megalotis and one juvenile Lepus californicus captured on the control plot, indicating a normally low frequency and incidental capture. The three most common species (Dipodomys merriami, Dipodomys microps, and Perognathus longimembris) accounted for $84.7 \%$ of the captured population on the burned site and only $54.9 \%$ on the unburned control (Table 4.6).

Table 4.5 Estimated densities (number/hectare \pm 2 SE) of srnall mammals on the BECAMP plots (1.67 ha) at the Mid Valley burn site, MID002 and MID003, during June 1988. Numbers in parentheses are individual animals captured.

\begin{tabular}{|c|c|c|c|c|}
\hline Species & \multicolumn{2}{|c|}{$\begin{array}{c}\text { MID002 } \\
\text { (Burn) }\end{array}$} & \multicolumn{2}{|c|}{$\begin{array}{l}\text { MIDO03 } \\
\text { (Control) }\end{array}$} \\
\hline DIP MER & $8.9 \pm 0$ & (15) & $3.4 \pm 1.2$ & (5) \\
\hline DIP MIC & $\overline{7}$ & (1) & $3.6 \pm 0$ & (6) \\
\hline PER LON & $3.6 \pm 0$ & (6) & $3.6 \pm 0$ & (6) \\
\hline PER FOR & $\overline{-}$ & (2) & $\bar{*}$ & (3) \\
\hline PER MAN & $*$ & (1) & $4.1 \pm 2.7$ & (5) \\
\hline REI MEG & $m-$ & & $\pi$ & (1) \\
\hline ONY TOR & $*$ & (1) & $*$ & (4) \\
\hline LEP CAL & -- & & * & (1) \\
\hline Total N/ha & 15.4 & (26) & 18.4 & (31) \\
\hline Total species & 6 & & 8 & \\
\hline
\end{tabular}

* Species present but data insufficient to calcuiate density. 
Table 4.6 Percent of total captured population (\%T) and sex ratio (M/F = male : female) of small mammals on the BECAMP plots in Mid Valley in 1988.

\begin{tabular}{|c|c|c|c|c|}
\hline \multirow[b]{2}{*}{ Species } & \multicolumn{2}{|c|}{$\begin{array}{c}\text { MIDO02 } \\
\text { (Burn) }\end{array}$} & \multicolumn{2}{|c|}{$\begin{array}{l}\text { MID003 } \\
\text { (Control) }\end{array}$} \\
\hline & $\% \mathrm{~T}$ & $\mathrm{M} / \mathrm{F}$ & $\% \mathrm{~T}$ & $\mathrm{M} / \mathrm{F}$ \\
\hline DIP MER & 57.7 & $1: 2.7$ & 16.1 & $1.5: 1$ \\
\hline DIP MIC & 3.8 & $1: 0$ & 19.4 & $1: 2$ \\
\hline PER LON & 23.2 & $1: 1$ & 19.4 & $1: 1$ \\
\hline PER FOR & 7.7 & $1: 1$ & 9.7 & $2: 1$ \\
\hline PER MAN & 3.8 & $0: 1$ & 16.1 & $4: 1$ \\
\hline REI MEG & $-\cdots$ & - & 3.2 & 1:0 \\
\hline ONY TOR & 3.8 & $0: 1$ & 12.9 & $1: 3$ \\
\hline LEP CAL & - & - & 3.2 & indet \\
\hline
\end{tabular}

In the case of Lepus californicus, only juveniles of indeterminate sex were captured.

A comparison of the densities and frequencies of species on these two plots indicated a greater species diversity on the unburned plot. On the burned plot, D. merriami and Perognathus longimembris accounted for $80.8 \%$ of the captured population, and most of these were captured on the edge of the plot nearest to the unburned vegetation; no more than two animals of the other species were captured.

The percentage of Dipodomys merriami was greater on the Mid Valley burned plot than on the unburned control plant $(57.7 \%$ and $16.1 \%$ ), while Dipodomys microps mostly disappeared (3.8\% and $19.4 \%$ ). Assuming that the unburned area was representative of the population on the burned area before the fire, the percent of the total estimated population of these two kangaroo rats $(D$. merriami at $16.1 \%$ and $D$. microps at $19.4 \%$ ) were approximately equal before the fire. These results are consistent with earlier studies which showed that $D$. merriami density increased in disturbed areas while D. microps decreased (Beatley 1976a; Allred and Beck 1963; Hunter and Medica 1989). This shift in kangaroo rat population has been attributed to a decrease in percent shrub cover from that preferred by $D$. microps (Beatley 1976a). Disturbed areas (e.g., fire, nuclear detonation) appear to have more annual herbs (see Status of Ephemerals in this report), which provide reproductive energy for $D$. merriami (Bradley and Mauer 1971, Van De Graaf and Balda 1973), while D. microps depends on leaves fror perennial shrubs (Beatley 1969; Kenagy 1973).

There was no significant difference (chi-square, $p>0.05$ ) between percent trap success on the two plots (10.9\% on the burned plot and $10.7 \%$ on the unburned control). A total of 26 different animals were captured 49 times on the burned plot for an average of 1.9 times per 
animal, while 31 different animals were captured 48 times on the unburned plot, averaging 1.6 .

The sex ratio of the most frequently captured species (Table 4.6) did not differ significantly from 1:1 on either plot. Adult Dipodomys merriami males on the burned area $(43.6 \pm 3.2 \mathrm{~g})$ were heavier than adult males captured on the unburned control $(41.0 \pm 2.0 \mathrm{~g})$, but not with any significance. There were no significant differences between adult weights of male and female Perognathus longimembris, although adult females were slightly heavier than adult males on both plots (Table 4B-4, Appendix 4B).

\section{Redrock Valley}

The estimated densities of the most commonly captured species and presence of other species on the burned and unburned plots in Redrock Valley during the three trapping periods are provided in Table 4.7. During the July trapping period, all species except Sylvilagus audubonii occurred on both plots; $S$. audubonii was captured on the edge of the control plot farthest from the burned area, which was also the slope of a wash. "Several rabbits and small mice were observed running ahead of the flames" (Fire Chief $H$. Hill, 22 July 1988). On 22 July 1988, BECAMP reconnoitered the area and the carcass of one S. audubonii was found on the burned area after the fire, indicating mortality in this species due to the fire. Also observed was an abundance of ant activity and several rodent burrows had been recently cleaned out, as evidenced by fresh sand in the blackened areas. Estimated densities of Dipodomys merriami were 1.3 times higher on the control than on the burn in July, but 1.1 and 1.2 times lower in August and October, respectively (Table 4.7). The estimated density for $D$. merriami on the unburned control plot remained the same from July through October. The estimated densities for this species remained fairly constant on the burned plot from August to October after an increase from July to August.

The number of Dipodomys microps on the burned plot increased one month after the fire but decreased slightly two months later. Beatley (1976a) showed that $D$. microps populations decreased or disappeared from areas which were disturbed as compared to nearby, undisturbed areas. However, the period of this occurrence was not known. Based on the data in Table 4.7, it appeared that $D$. microps numbers were low on the burn plot immediately after the fire (perhaps due to emigration, mortality or nonemergence from burrows) but increased one month later. No animals marked on the unburned area were found on the burned area until October. This suggested that the initial increase in D. microps was due to increased activity of animals already residing on the burned plot. It is unclear whether or not the regular supply of food from the bait drew more animals in from the outside edges of the plot. The home range of these rodents might have increased with the need to go farther in search of food (White and Allred 1961; Allred and Beck 1963). Maza et al. (1973) reported that home ranges of heteromyid rodents were smallest during years of poorest conditions for plant growth, with animals becoming torpid when energy supplies were inadequate. Activity and reproductive patterns might also have been responsible for 


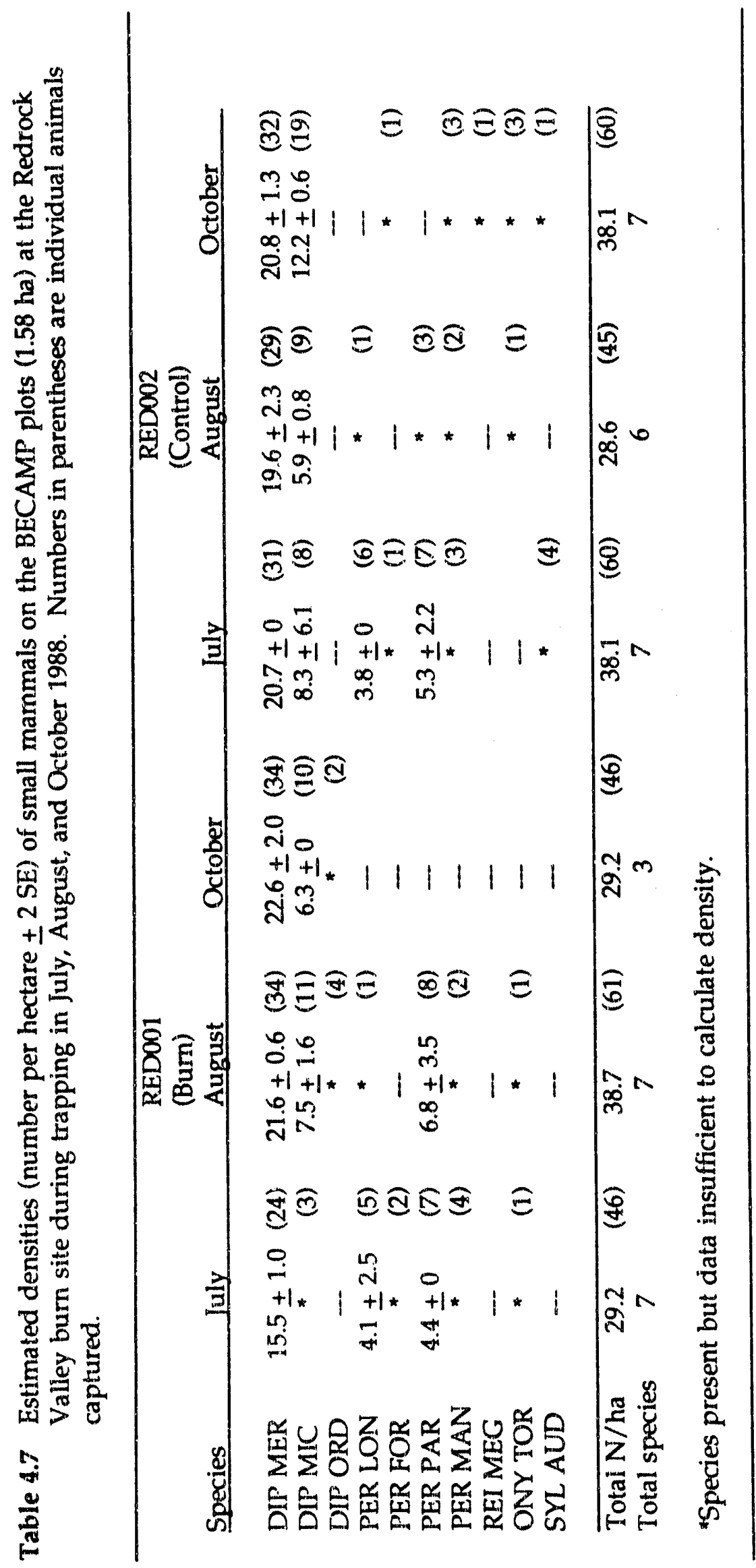


the fluctuating density of D. microps on the control plot (Allred and Beck 1963).

The most significant change in species composition occurred during August trapping when Dipodomys ordii appeared on the burned plot for the first time. D. ordii previously had been collected most frequently on the Nevada Test Site in areas disturbed by aboveground nuclear testing (Jorgensen and Hayward 1965). Where these animals emigrated from is unknown since they were not ever captured on the unburned control plot in 1988, nor were they trapped on the burned plot in July. It is possible that these animals were present in low numbers and relatively inactive at the trapping period due to temporal or seasonal activity patterns (Jorgensen et al. 1980; Whitford 1974). D. ordii was trapped in this valley during 1959-1965 sampling by BYU (Jorgensen \& Hayward 1965). This species was not captured at any other BECAMP study location on the NTS during the 1987 and 1988 trapping seasons.

Heteromyid rodents (Dipodomys and Perognathus) accounted for $89.1 \%$ of the captured individuals in July on the Redrock Valley burn plot, $95.1 \%$ in August, and $100 \%$ in October (Table 4.8). These same rodents accounted for $88.4 \%, 93.4 \%$ and $86.6 \%$ of the captured population on the control plot in July, August, and October, respectively. By October, most of the Perognathus spp. had disappeared due to seasonal hibernation. The sex ratios for the most commonly captured species on both plots (Table 4.8 ) were not significantly different from a 1:1 ratio of males to females.

Percent trap successes on the Redrock Valley burned area for July and October (months with 3 trap nights) were $22.9 \%$ and $24.8 \%$, respectively and $25.0 \%$ and $31.4 \%$ on the unburned control. Animals on the burned area were captured an average of 2.1 times (46 animals captured 96 times) in July, while on the unburned control plot in July the average capture was 1.8 times (60 captured 105 times). In October, average captures were over 2.0 for both plots (2.4 times in the burned area and 2.2 times on the control). Trapping effort in August was only 280 trap nights as opposed to 420 in July and October; therefore, the percent trap success (33.9\% on the burned plot and $25.0 \%$ on the unburned control) and average capture numbers (1.6 times on both plots) for August are not readily comparable to the other two months in which trapping occurred.

Among Dipodomys merriami in July, ANOVA on mean weights (Table 4B-2, Appendix 4B) revealed that, while adult females on the control $(43.1 \pm 1.9 \mathrm{~g}, \mathrm{n}=11)$ were consistently heavier than adult females on the burned area $(39.3 \pm 2.6 \mathrm{~g}, \mathrm{n}=5)$, the difference was not statistically significant ( $p>0.05$ ). The mean weight of males on the control $(40.0 \pm 3.2 \mathrm{~g}, \mathrm{n}=$ 11) also did not differ significantly from that of males on the burned area in July $(41.3 \pm 3.3$ $g, n=8$ ). In July and August, there were no significant differences between male and female adult $D$. merriami on the control $(42.2 \pm 2.6 \mathrm{~g}, \mathrm{n}=10$ and $43.7 \pm 2.2 \mathrm{~g}, \mathrm{n}=11)$; however, in August, male adults were significantly heavier $(0.01<p<0.02)$ than females on the burned area $(43.4 \pm 1.9 \mathrm{~g}, \mathrm{n}=13$ and $39.4 \pm 2.6 \mathrm{~g}, \mathrm{n}=12)$. Control adult females were also significantly heavier than adult females from the burned area in August $(p<0.05)$. Males were consistently 


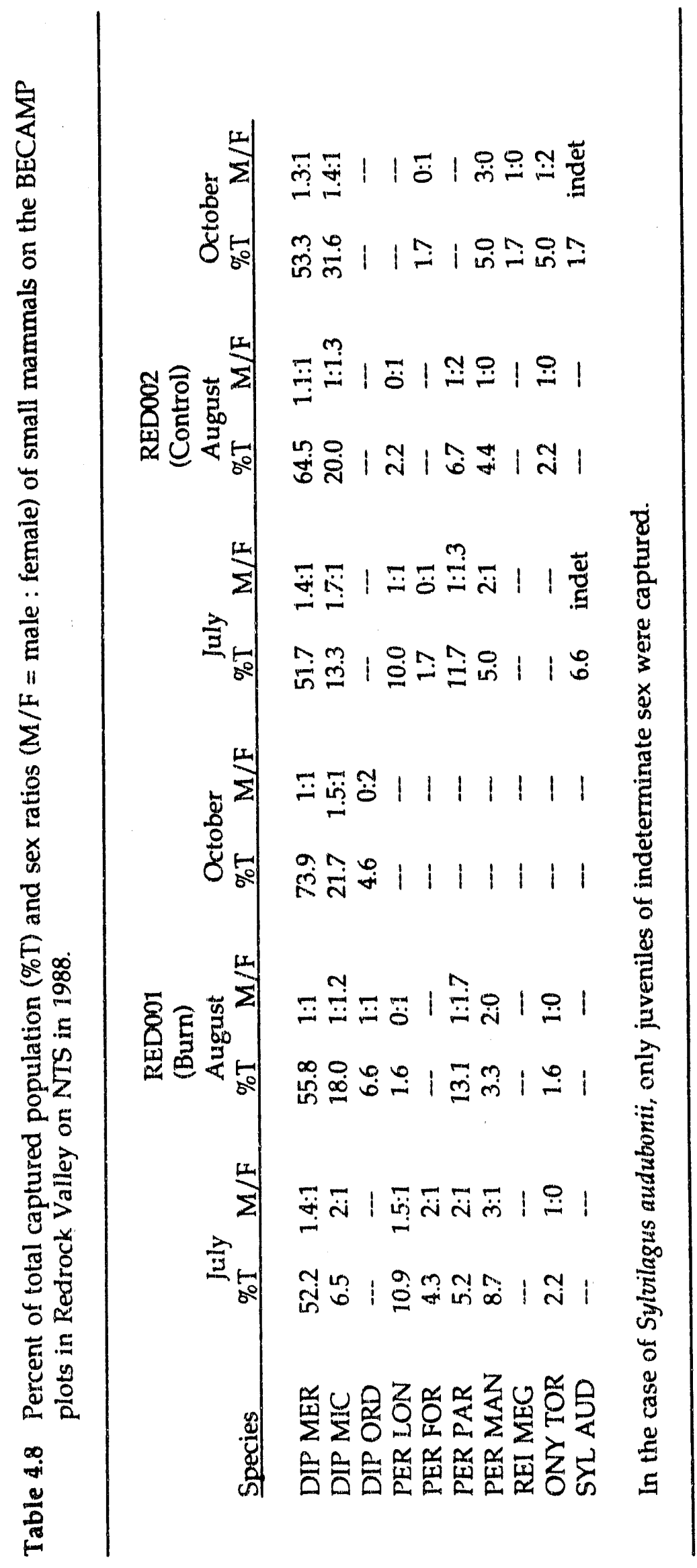


heavier than females on the burned area in all three months, while females on the control were heavier than males in July and August, but lighter in October. After trapping in October, adult male D. merriami captured on both areas were slightly heavier than adult females.

The mean weight of adult female $D$. merriami on the control decreased significantly (ANOVA, $\mathrm{p}<0.05)$ from August to October $(43.7 \pm 2.2 \mathrm{~g}, \mathrm{n}=11$, to $37.1 \pm 1.5 \mathrm{~g}, \mathrm{n}=12)$ after relatively no gain from July $(43.1 \pm 1.9 \mathrm{~g}, \mathrm{n}=10)$. While the mean weight of adult male $D$. merriami on the control also decreased from August to October $(42.2 \pm 2.6 \mathrm{~g}, \mathrm{n}=10$, to $38.2 \pm 1.7 \mathrm{~g}$, $n=17)$, mean weight increased slightly after July $(40.0 \pm 3.2 \mathrm{~g}, \mathrm{n}=11)$, but without any statistical significance. Mean weights for adult males on the burned plot showed similar changes over the three trapping periods, with a significant decrease in mean weight from August to October (ANOVA, $\mathrm{p}<0.05$ ). The mean weights of adult females on the burned plot remained relatively constant throughout the duration of trapping in 1988, with no significant changes in mean weight. ANOVA on the mean weights of Dipodomys microps and Perognathus longimembris revealed no significant changes in mean weight between the three trapping times (Tables 4B-2 and 4B-3, Appendix 4B). Changes in weight, along with morphological changes, can be indicators of reproduction in the population (Bradley \& Mauer 1971; Kenagy 1973). No real conclusions can be made on whether or not these two plots had differing reproductive success, as not all BECAMP personnel indicated reproductive condition (including non-reproductive status) on the data sheets.

\section{Rock Valley (Fenced, Previously Irradiated Plot and Control)}

This area on the southern edge of the Nevada Test Site contains a 9-hectare fenced plot B (ROV002) that was continuously exposed to gamma-radiation from a ${ }^{137} \mathrm{Cs}$ source over a period of 17 years from 1964 to 1981, and an unfericed 9-ha control plot D (ROV004) approximately 200 meters to the west. BECAMP sampled $12 \times 12$ grids on the southwestern edge of both of these original plots (ROV007 on plot D and ROV008 on plot B) on 14-17 June 1988 in order to compare present populations to those sampled in earlier studies (e.g., French et al. 1974; Turner 1973, 1974). The southwestern portion of plot D, IBP plot 16, has historical data from the 1970's, so a comparison of present and past populations was possible.

The estimated densities of the most common species captured on these two plots during 1988 and on IBP plot 16 during July 1972, April 1973, and August 1973 are shown in Table 4.9. Species not in the fenced plot but present in the control were Perognathus longimembris and Peromyscus maniculatus. P. longimembris was reported in both areas in 1964 (French et al. 1974) and 1966 (French et al. 1967) but in much lower numbers in the fenced plot after exposure to radiation as compared to the unfenced, non-irradiated area. Habitat for $P$. longimembris appeared to be less than optimum inside of the enclosure - rocky rather than a sandy soil surface. French et al. (1974) reported higher perennial production (leaf and seed) 
Table 4.9 Estimated densities (number/ha $\pm 2 \mathrm{SE}$ ) of small mammals inside the 3.24-ha Rock Valley fenced plot ( $3.24 \mathrm{ha}$ ) in June 1988 and on IBP plot 16 in July 1972, April and August 1973, and June 1988. Numbers in parentheses are individual animals captured.

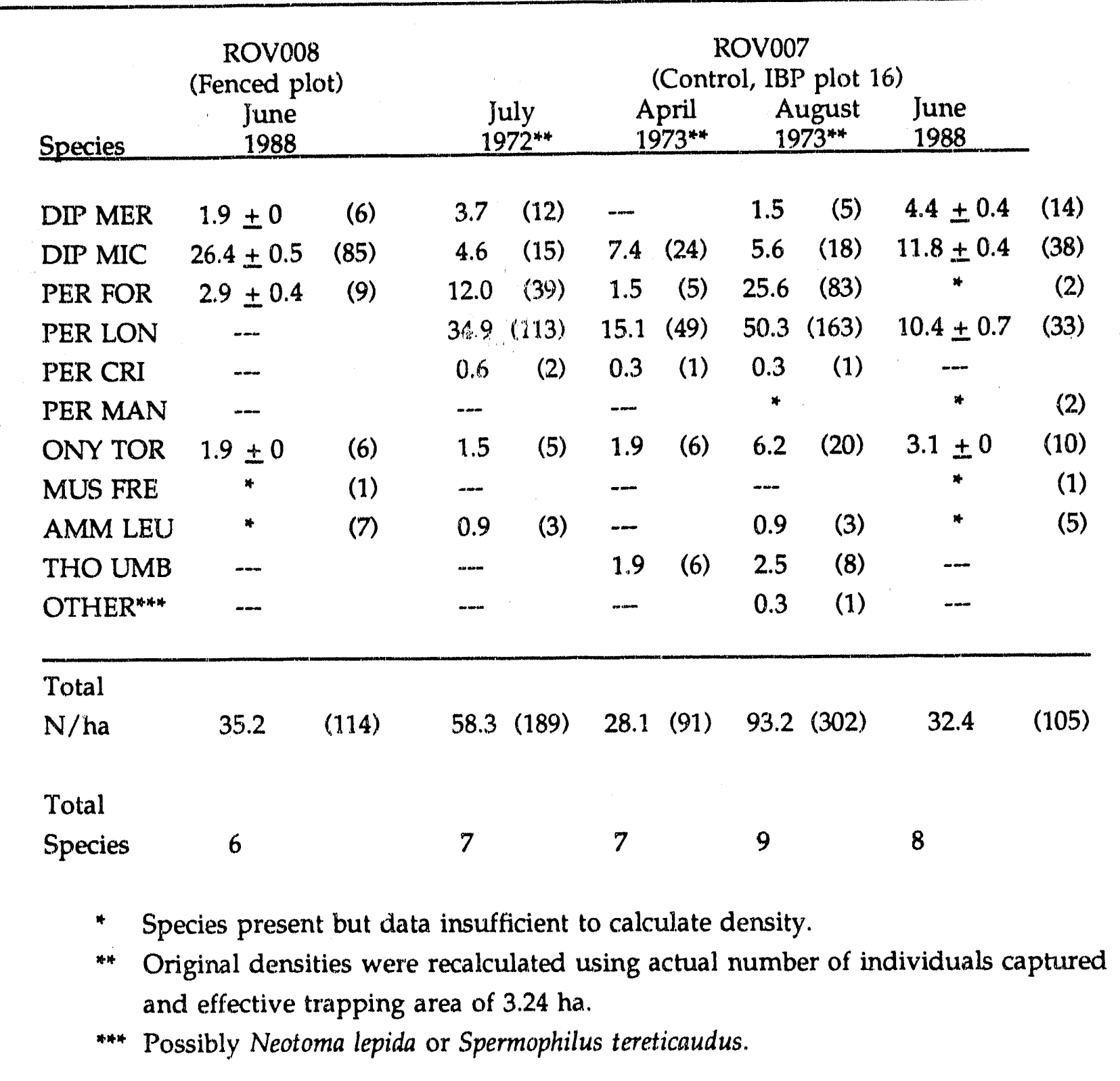

in the enclosed plot, while the unfenced plot had a greater annual seed supply. While $P$. longimembris is a granivore, it mainly sifts through the soil for seeds from annual or perennial grasses and forbs (Zeveloff and Collett 1988).

Estimated densities of species in the unfenced control (IBP plot 16) in July of 1972 and April 
and August of 1973 were lower for Dipodomys merriami $(1.5-3.7 /$ ha $)$ and Dipodomys microps (4.6 - 7.4/ha) than in 1988 and higher for Perognathus longimembris $(15.1-50.3 / \mathrm{ha})$ and Perognathus formosus $(1.5$ - 25.6) (Turner 1973, 1974). The two species of Dipodomys initially did not survive well in the irradiated enclosure. Both species were present in low numbers and were extirpated less than one year after initiation of radiation treatment. D. microps died out after five years in a fenced, unirradiated control plot of the same size while kangaroo rats in the remaining enclosures declined to only two animals but persisted in low numbers until at least 1970 (French et al. 1974). This indicated that the enclosure, more so than the gamma radiation, might have contributed to the decline in the kangaroo rat population. Less than optimum food supplies was also a contributing factor - a higher perennial leaf and seed production in 1966 to 1968 within plot B, which favors D. microps reproduction, and higher annual seed production on plot 16 , which favors $D$. merriami reproduction, but low seasons of primary productivity in 1963,1964, and 1967 showed corresponding declines in the populations of kangaroo rats (French et al. 1974). French et al. (1974) also concluded that chronic exposure to the gamma radiation was clearly detrimental to a desert rodent population in terms of a decrease in the probability of survival.

Trap success was higher inside of the fenced area ( $30.9 \%$ as compared to $24.5 \%$ ) as was the average times captured ( 2.3 and 2.0 ). This might be due to a smaller home range of some of the animals in the fenced plot, which is inversely related to density (Maza et al. 1973).

The most significant find on both of these plots in 1988 was the presence of the long-tailed weasel, Mustela frenata. Rock Vauley has the most continuous sampling history of any plot on NTS, and never before has this species been encountered in this valley (Medica 1990). Peromyscus crinitus was captured in 1972 on plot 16, but not in 1988. Peromyscus maniculatus, however, was captured in 1988 on plot 16, but not reported in 1972. Both species were reported in this area by BYU (Jorgensen and Hayward 1965).

Sex ratios did not differ significantly from a 1:1 ratio of male to females (Table 4.10). Species composition on the two plots, however, was slightly different with two more species captured on plot 16 , one of which was Perognathus longimembris. Of the animals captured in the fenced plot, $79.8 \%$ were Dipodomys merriami and Dipodomys microps, while only $49.5 \%$ of the animals captured on plot 16 were of these two species (Table 4.10). The remainder of the animals captured on plot 16 were mostly $P$. longimembris, which was absent from inside of the fenced plot.

Adult female Dipodomys merriami in the fenced plot were $10.9 \%$ heavier than males in the fenced area (ANOVA, $\mathrm{p}=0.008$ ) and $25.5 \%$ heavier than females in the unfenced area (ANOVA, $\mathrm{p}=0.002$ ). The mean weight (Table 4B-2, Appendix $4 \mathrm{~B}$ ) of males in the unfenced area was not significantly heavier than that of females on the fenced plot $(p>0.05)$, and there 
Percent of total captured population $(\% \mathrm{~T})$ and sex ration $(\mathrm{M} / \mathrm{F}=$ male : female) of small mammals ir the Rock Valley fenced plot (ROV008) in 1988 and on IBP plot 16 (ROV VO7) in 1972, 1973, and 1988.

\begin{tabular}{|c|c|c|c|c|c|c|c|}
\hline \multirow[b]{2}{*}{ Species } & \multicolumn{2}{|c|}{$\begin{array}{c}\text { ROV008 } \\
\text { (Fenced Plot) } \\
\text { June } \\
1988\end{array}$} & $\begin{array}{l}\text { July } \\
1972\end{array}$ & $\begin{array}{c}\text { ROV007 } \\
\text { (Control, IBP plot 16) }\end{array}$ & $\begin{array}{l}\text { lot 16) } \\
\text { August } \\
1973\end{array}$ & \multicolumn{2}{|c|}{$\begin{array}{l}\text { June } \\
1988\end{array}$} \\
\hline & $\% \mathrm{~T}$ & $\mathrm{M} / \mathrm{F}$ & $\% \mathrm{~T}$ & $\% \mathrm{~T}$ & $\% \mathrm{~T}$ & $\% \mathrm{~T}$ & $M / F$ \\
\hline DIP MER & 5.3 & $1: 1$ & 6.3 & -- & 1.7 & 13.3 & $1.8: 1$ \\
\hline DIP MIC & 74.5 & $1: 1.1$ & 7.9 & 26.4 & 6.0 & 36.2 & $1.1: 1$ \\
\hline PER FOR & 7.0 & $* 1: 1$ & 20.6 & 5.5 & 27.5 & 1.9 & $0: 2$ \\
\hline PER LON & - & - & 59.8 & 53.8 & 54.0 & 31.4 & $1.4: 1$ \\
\hline PER CRI & - & - & 1.1 & 1.1 & 0.3 & -- & -- \\
\hline PER MAN & -- & $\cdots$ & -- & - & --- & 1.9 & $1: 1$ \\
\hline ONY TOR & 5.3 & $* 4: 1$ & 3.7 & 6.6 & 6.6 & 9.5 & $2.3: 1$ \\
\hline MUS FRE & 0.9 & $0: 1$ & -- & $-\cdots$ & -- & 1.0 & $0: 1$ \\
\hline AMM LEU & 6.1 & ${ }^{*} 1: 4$ & 1.6 & - & 1.0 & 4.8 & $1.5: 1$ \\
\hline THO UMB & -- & - & -- & 6.6 & 2.6 & -- & - \\
\hline OTHER & -- & - & -- & - & 0.3 & -- & -- \\
\hline
\end{tabular}

* One animal of undetermined sex not included.

was no significant difference between the mean weight of adult males in the enclosure and the mean weright of actult nrates in the unfencectarea. Anctut mate-Bipotomys microps were heavier than adult females on both plots (Table 4B-3, Appendix 4B), but only significantly so in the enclosed plot $(p<0.05)$, while males and females from the unfenced area were heavier than males and females from the fenced plot, but not with any significance.

\section{Disturbance From Aboveground Testling and Blading}

Two sites in Yucca Flat were trapped in 1988 to estimate the current population densities and species compositions of areas that had been denuded by the heat, fire and radiation from repeated aboveground nuclear testing during the 1950's. Site T1, on the western side of Yucca Flat, was the location of four tower-supported tests between 1952 and 1957. A circular area of approximately 500 hectares was completely cleared of vegetation due to the testing, and has since been invaded by Salsola spp., Stipa speciosa, Bromus rubens, and Bromus tectorum. This area was sampled for small mammals and other animals by BYU during continuous studies from 1959 to 1965 (Jorgensen and Hayward 1965). BECAMP plots were placed on an 
existing BYU trap line to the southeast (SE) of ground zero (GZ). An $8 \times 8$ grid (YUF009) was set up on this line $960 \mathrm{~m}(3168 \mathrm{ft}$ ) SE of GZ to sample the ciisturbed area. Another $8 \times 8$ grid (YUF010) $1760 \mathrm{~m}$ (5808 ft) SE of GZ on this line was established as i control in an undisturbed Grayia-Lycium plant community (as defined by Beatley 1976a).

Trappinf, for nocturnal mammals took place on 4, 16, 17, and 18 May and 9, 10 August in 1988. /sugust trapping was done to assess summer resident species and the results were not inclucied in the density estimation. By August, most of the nocturnal rodents were inactive, with the exception of Vipodomys merriami, (which was still abundant at both sites (Appendix 4C, p. 142. However, two additional species, Ammospermophilus leucurus and Reithrodontomys megalotis, were captured on the blast area in August while both had been captured only on the control during trapping in May.

The estimated spring densities of the most commonly captured species and presence or absence of other species on $\mathrm{T} 1$ and its control are shown in Table 4.11.

Table 4.11 Estimated densities (number/ha $\pm 2 \mathrm{SE}$ ) of small mammals on the BECAMP subsidiary plots (1.44 ha) at the tower shot area, T1, in Yucca Flat in May 1988. Numbers in parentheses are individual animals captured.

\begin{tabular}{|c|c|c|c|c|}
\hline \multirow[b]{2}{*}{$\begin{array}{l}\text { Species } \\
\text { DIP MER } \\
\text { DIP MIC } \\
\text { PER LON } \\
\text { PER FOR } \\
\text { PER MAN } \\
\text { REI MEG } \\
\text { ONY TOR } \\
\text { THO UMB } \\
\text { AMM LEU }\end{array}$} & \multicolumn{2}{|c|}{$\begin{array}{l}\text { YUF009 } \\
\text { T1 Blast Area } \\
(960 \mathrm{~m} \mathrm{SE} \mathrm{GZ)}\end{array}$} & \multicolumn{2}{|c|}{$\begin{array}{c}\text { YUF010 } \\
\text { Control } \\
(1760 \mathrm{~m} \mathrm{SE} \mathrm{GZ)} \\
\end{array}$} \\
\hline & $\begin{array}{c}19.6 \pm 2.0 \\
5.0 \pm 0.6 \\
* \\
* \\
=\end{array}$ & $\begin{array}{l}(27) \\
(7) \\
(1) \\
(4) \\
(1) \\
(1)\end{array}$ & $\begin{array}{c}14.8 \pm 4.8 \\
3.9 \pm 1.5 \\
27.3 \pm 2.1 \\
= \\
= \\
=\end{array}$ & $\begin{array}{l}\text { (4) } \\
\text { (3) } \\
\text { (3) }\end{array}$ \\
\hline $\begin{array}{l}\text { Total N/ha } \\
\text { Total species }\end{array}$ & $\begin{array}{c}28.5 \\
6\end{array}$ & $(41)$ & $\begin{array}{c}48.6 \\
6\end{array}$ & $\overline{(70)}$ \\
\hline \multicolumn{5}{|c|}{ Species present but data insufficient to calculate density. } \\
\hline
\end{tabular}

As has been shown in other disturbed areas, the captured population of Kangaroo rats consisted entirely of Dipodomys merriami on the disturbed plot and both D. merriami and Dipodomys microps on the control plot. Species unique to the control area from 1959 to 1965 but found on both plots in 1988 were Peromyscus maniculatus, Perognathus longimembris, and Perognathus formosus. Perognathus parous and Peromyscus crinitus were found only in the 
control area during BYU sampling but were not captured during the two sampling periods in 1988 on either plot. D. microps was found on both areas in 1959 to 1965, and only on the control in 1988. Reithrodontomys megalotis was captured in both plots for the first time in 1988. The only species unique to the disturbed area during the history of sampling on this site was the gopher', Thomonys umbrinus. However, aboveground evidence suggests that this species was present on the undisturbed control in recent times, but was not captured in the Sherman traps or is no longer present. Dipodomys ordii was captured on the blast area by BXU (1959-1965) but was not captured in 1988 (Jorgensen and Hayward 1965).

Of the captured animals on the blast area, $65.9 \%$ were Dipodomys merriami, while Perognathus longimembris (at 54.3\%) was the most frequently captured species on the control (Table 4.12). Average capture rat in the blast area ( 41 animals captured 101 times for an average of 2.5) was 1.3 times greater than that on the conirol area (70 animals captured 133 times for an average of 1.9). This, again, is probably due to differences in abundance of food resources, home ranges, and population densities between the two plots. Percent trap success on the T1 blast area, like that on the Redrock bum disturbed study area, was lower than that on the control (19.7 and $26.0 \%$ respectively).

Table 4.12 Percent of total captured population (\% $\mathrm{T})$ and sex ratio $(\mathrm{M} / \mathrm{F}=$ male : female) of small mammals on the BECAMP plots at T1, Yucca Flat in 1988.

\begin{tabular}{|c|c|c|c|c|}
\hline \multirow[b]{2}{*}{ Species } & \multicolumn{2}{|c|}{$\begin{array}{l}\text { YUJF009 } \\
\text { T1 }\end{array}$} & \multicolumn{2}{|c|}{$\begin{array}{l}\text { YUF010 } \\
\text { Control }\end{array}$} \\
\hline & $\% \mathrm{~T}$ & $M / F$ & $\% \mathrm{~T}$ & $M / F$ \\
\hline DIP MER & 65.9 & $1.7: 1$ & 24.3 & $1.8: 1$ \\
\hline DIP MIC & - & - & 7.1 & $4: 1$ \\
\hline PER LON & 17.1 & $1: 1.7$ & 54.3 & $1: 1.2$ \\
\hline PER FOR & 2.4 & $1: 0$ & - & - \\
\hline PER MAN & 9.8 & $1: 3$ & - & - \\
\hline REI MEG & - & - & 5.7 & 1:1 \\
\hline ONY TOR & 2.4 & $0: 1$ & 4.3 & $2: 1$ \\
\hline THO UMB & 2.4 & $0: 1$ & - & - \\
\hline AMM LEU & $\cdots$ & $\cdots$ & 4.3 & $3: 0$ \\
\hline
\end{tabular}

Indeterminate values indicate that only one sex of a species was captured.

Sex ratios for the most commonly captured animals (Table 4.12) on the two plots did not differ significantly from 1:1. The mean weight of adult male Dipodomys merriami on the blast area $(44.1 \pm 1.5 \mathrm{~g}, \mathrm{n}=16)$ was slightly heavier than that of adult females $(42.6 \pm 1.9 \mathrm{~g}, \mathrm{n}=9)$ but not significantly different from adult males of the control $(43.3 \pm 1.6 \mathrm{~g}, \mathrm{n}=11)$. Female mean weights did not differ significantly between plots (Table 4B-2, Apperidix 4B). Mean 
weights of adult males, however, were slightly heavier than females on YUF009 $(0.05<\mathrm{p}<0.10)$ and YUF010 $(0.02<\mathrm{p}<0.05)$ after summer trapping in August. The mean weights of adult male $(7.7 \pm 0.3 \mathrm{~g}, \mathrm{n}=17)$ and female $(8.1 \pm 0.4 \mathrm{~g}, \mathrm{n}=21)$ Perognathus longimembris were greater on the control than that of male $(7.3 \pm 0.5 \mathrm{~g}, \mathrm{n}=5)$ and female $(7.6 \pm 0.5 \mathrm{~g}, \mathrm{n}=2)$ $P$. longimembris on the blast area but not significantly heavier (Table 4B 4 , Appendix $4 \mathrm{~B}$ ).

Another aboveground test area sampled by BECAMP in 1988 was the T3 area. This area was aiso used for tower-supported nuclear tests from 1952 to 1957 and is located on the eastern side of Yucca Flat. To study the long-term effects of aboveground testing in this area, a $14 \times$ 5 grid was located approximately $1000 \mathrm{~m}$ (3300 ft) southeast of GZ (YUF013). A $14 \times 5$ grid control plot (YUF012) was set up approximately $300 \mathrm{~m}$ east of the disturbed area plot. The $3 \mathrm{~B}$ waste consolidation site was located immediately to the north of the T3 control area and was surrounded by a barbed and chicken wire fence. This area was "cleaned up" in 1987, which involved removal of all radioactive scrap material previously stored on the site as well as blading and removal of several inches of surface soil, and hence all vegetation. A $14 \times 5$ grid was also established on this cleared site (YUF011). Mammals were censused on all three plots on 20, 25, and 26 May 1988.

The estimated densities for all three sites are shown in Table 4.13. Dipodomys merriami and

Table 4.13 Estimated densities (number/hectare $\pm 2 \mathrm{SE}$ ) of small mammals on the BECAMP plots (1.58 ha) in Yucca Flat, YUF011, YUF012, and YUF013, in May 1988. Numbers in parentheses are number of individual animals captured.

\begin{tabular}{|c|c|c|c|c|c|c|}
\hline \multirow{2}{*}{$\begin{array}{l}\text { Species } \\
\text { DIP MER } \\
\text { DIP MIC } \\
\text { PER LON } \\
\text { PER MAN } \\
\text { ONY TOR } \\
\text { AMM LEU }\end{array}$} & \multicolumn{2}{|c|}{$\begin{array}{l}\text { YUF011 } \\
\text { 3B Consolidation Site } \\
\text { (bladed area) }\end{array}$} & \multicolumn{2}{|l|}{$\begin{array}{l}\text { YUF012 } \\
\text { Control }\end{array}$} & \multicolumn{2}{|c|}{$\begin{array}{c}\text { YUF013 } \\
\text { T3 Blast Area } \\
\text { (1000m SSE GZ) }\end{array}$} \\
\hline & $\begin{array}{c}5.2 \pm 0.5 \\
* \\
- \\
- \\
-\end{array}$ & $\begin{array}{l}(8) \\
(1) \\
(2)\end{array}$ & $\begin{array}{c}10.8 \pm 0 \\
4.1 \pm 1.1 \\
12.6 \pm 1.7 \\
* \\
*\end{array}$ & $\begin{array}{r}(17) \\
(6) \\
(19) \\
(2) \\
(1) \\
(7)\end{array}$ & $\begin{array}{c}8.5 \pm 1.0 \\
\cdots \\
\cdots \\
\cdots \\
\cdots\end{array}$ & (1) \\
\hline $\begin{array}{l}\text { Total N/ha } \\
\text { Total species }\end{array}$ & $\begin{array}{c}7.0 \\
3\end{array}$ & $(11)$ & $\begin{array}{c}33.1 \\
6\end{array}$ & $(52)$ & $\begin{array}{c}10.2 \\
3\end{array}$ & (6) \\
\hline
\end{tabular}

Perognathus longimembris made up $90.9 \%$ and $87.5 \%$ of the animals captured on $3 B$ and T3 respectively, while these two species comprised $69.2 \%$ of the captured animals on the control (Table 4.14). Both of the disturbed areas showred the typical decrease in numbers or absence 
of Dipodomys microps as compared to the control. Animals on the 3B site were never captured more than $30 \mathrm{~m}$ in from the north and east edges of the plot, which were closest to the undisturbed vegetation. Animals on the other two plots were captured throughout the plot. Even though the control was only $15 \mathrm{~m}$ from the bladed area, no animals from the control were ever captured on the bladed area. However, one Ammospermophilus leucurus did move from the control to the $\mathrm{T} 3$ site.

Table 4.14 Percent of total captured population $(\% \mathrm{~T})$ and sex ratio $(\mathrm{M} / \mathrm{F}=$ male : fernale) on BECAMP plots YUF011, YUF012, and YUF013 on Yucca Flat in 1988.

\begin{tabular}{|c|c|c|c|c|c|c|}
\hline \multirow[b]{2}{*}{ Species } & \multicolumn{2}{|c|}{$\begin{array}{l}\text { YUF011 } \\
\text { (3B Consolidation) }\end{array}$} & \multicolumn{2}{|c|}{$\begin{array}{l}\text { YUF012 } \\
\text { (Control) }\end{array}$} & \multicolumn{2}{|c|}{$\begin{array}{c}\text { YUF013 } \\
\text { (T3 Blast Area) }\end{array}$} \\
\hline & $\% \mathrm{~T}$ & $\mathrm{M} / \mathrm{F}$ & $\% \mathrm{~T}$ & $\mathrm{M} / \mathrm{F}$ & $\% \mathrm{~T}$ & $\mathrm{M} / \mathrm{F}$ \\
\hline DIP MER & 72.7 & $1: 1$ & 32.7 & $1.1: 1$ & 81.3 & $1.6: 1$ \\
\hline DIP MIC & 9.1 & $1: 0$ & 11.5 & $1: 2$ & $\ldots$ & - \\
\hline PER LON & 18.2 & $0: 2$ & 36.5 & $1: 1.4$ & 6.2 & $2: 0$ \\
\hline PER MAN & $-\cdots$ & $-\cdots$ & 3.9 & $0: 2$ & -- & - \\
\hline ONY TOR & - & - & 1.9 & $1: 0$ & -- & - \\
\hline AMM LEIJ & - & - & 13.5 & $1: 1.3$ & 12.5 & $2: 0$ \\
\hline
\end{tabular}

During the three nights of trapping, a total of 25,93 , and 36 animals were captured on 3B, the control, and $\mathrm{T} 3$ respectively, an average of $2.3,1.8$, and 2.3 times. Percent trap success was greatest on the control $(22.1 \%)$ as compared to $\mathrm{T} 3(8.6 \%)$ and $3 \mathrm{~B}(6.0 \%)$. Sex ratios of the most commonly captured species did not differ significantly from 1:1.

Adult female Dipodomys merriami on the control were not significantly heavier than males $(48.27 \pm 6.0 \mathrm{~g}, \mathrm{n}=8$ and $45.0 \pm 2.3 \mathrm{~g}, \mathrm{n}=8$, Table $4 \mathrm{~B}-2$, Appendix 4B), while adult males on the $3 B$ plot were slightly heavier $(0.05<\mathrm{p}<0.10)$ than the adult females of the $3 B$ plot $(45.7 \pm$ $0.7 \mathrm{~g}, \mathrm{n}=4$ and $42.8 \pm 2.6 \mathrm{~g}, \mathrm{n}=4)$. There was no significant difference between mean weights of male $(41.3 \pm 1.3 \mathrm{~g}, \mathrm{n}=8)$ and female $(41.8 \pm 4.9 \mathrm{~g}, \mathrm{n}=5)$ D. merriami on the T3 site. Adul: males on the control were significantly heavier than adult males on the $T 3$ site (ANOVA, $p<0.05$ ) but not the $3 B$ site. Adult male $D$. merriami captured on the $3 B$ site were also significantly heavier than males at the $T 3$ site (ANOVA, $\mathrm{p}<0.05$ ). The combined adult male and female mean weight on the control plot, YUF012, was significantly heavier than the combined mean weight of adult $D$. merriami on $T 3$, but not between any other plots (ANOVA, Student-Newman-Keuls range test, $\mathrm{p}<0.05$ ). 


\section{Continued Monitoring of Disturbance From the Sedan Cratering Test}

The Sedan nuclear detonation, conducted at the NTS on July 6, 1962, was unique in that ecological studies were conducted by BYU, in cooperation with the University of California, Los Angeles (UCLA), before and after detonation to determine the immediate close-in effects on small mammals (Allred et al. 1964). In 1988, BECAMP set up three plots on the 16A, $57^{\circ} 30^{\prime}$ azimuth established by BYU for the 1962 study. BYU trapped mammals on grids of known size at the following distances in feet from GZ: $1000(303 \mathrm{~m}), 2000(606 \mathrm{~m}), 3000$ $(909 \mathrm{~m}), 4000(1212 \mathrm{~m}), 5000(1515 \mathrm{~m}), 7000(2121 \mathrm{~m})$ and $9000(2727 \mathrm{~m})$. BECAMP study plots $(8 \times 8$ grids) were set up at the following distanced in feet from GZ: $1500(455 \mathrm{~m}$, YUF016), 3500 (1060 m, YUF017) and 5250 (1591 m, YUF018). Trapping occurred on 12, 13, and 14 July 1988, so that comparisons between the two data sets were possible. Differences in methods used to estimate population might, however, compromise the comparisons; BYU used a method described by Hayne (Allred, et al. 1964). This method is similar to Seber's, in that the population estimate, $P$, is directly proportional to the product of the total marked population (M) and the number of animals captured during the last trap period (C), and indirectly proportional to the number of recaptured animals in the last trap period $(R)$ or: $P=\frac{C \cdot M}{R}$. Studies in this area were also conducted by $O^{\prime}$ Farrell and Sauls (1987). The percent cover for 1988 at 303, 909, and $1515 \mathrm{~m}$ from GZ are found in the Status of Perennial Vegetation and Status of Desert Ephemeral Plants sections of this report. Percent vegetation before detonation is found in Allred et al. (1964); the blast damage extended to about 4500 feet $(1363 \mathrm{~m})$ from $\mathrm{GZ}$, so the $1591-\mathrm{m}$ plot can be considered an undisturbed control. The estimated density ('Table 4.15) and percent of total captured population (Table 4.16) of

Table 4.15 Estimated densities (number/hectare $\pm 2 \mathrm{SE}$ ) of small mammals on the BECAMP plots (1.44 ha) at Sedan crater, YUF01 $\overline{6}$, YUF017, and YUF018 during July 1988. Numbers in parentheses are number of individuals captured.

\begin{tabular}{|c|c|c|c|c|c|c|}
\hline \multirow{2}{*}{$\begin{array}{l}\text { Species } \\
\text { DIP MER }\end{array}$} & \multicolumn{2}{|c|}{$\begin{array}{c}\text { YUF016 } \\
\text { (455 m from GZ) }\end{array}$} & \multicolumn{2}{|c|}{$\begin{array}{c}\text { YUF017 } \\
\text { (1060 m from GZ) }\end{array}$} & \multicolumn{2}{|c|}{$\begin{array}{l}\text { YUF018 } \\
\text { (1591 m from GZ) }\end{array}$} \\
\hline & $23.7 \pm 2.6$ & (32) & $20.1 \pm 0$ & (29) & $10.8 \pm 1.2$ & (15) \\
\hline DIP MIC & $\ldots$ & & -- & & $5.8 \pm 0.9$ & (8) \\
\hline PER FOR & * & (1) & $\cdots$ & & -- & \\
\hline PER LON & $\ldots$ & & - & & $4.3 \pm 0.7$ & (6) \\
\hline AMM LEU & $*$ & (1) & $*$ & (2) & $4.2 \pm 0$ & (6) \\
\hline ONY TOR & $-\cdots$ & & $*$ & (1) & $\bar{*}$ & (4) \\
\hline SYL AUD & - & & $*$ & (1) & $*$ & (1) \\
\hline Total N/ha & 23.6 & (34) & 22.9 & (33) & 27.8 & $(40)$ \\
\hline Total species & 3 & & 4 & & 6 & \\
\hline
\end{tabular}

"Species present but data insufficient to calculate density. 
Table 4.16 Percent of total captured population (\% $\mathrm{T})$ and sex ratio $(\mathrm{M} / \mathrm{F}=$ male : female) of small mammals on the BECAMP plots at Sedan crater in Yucca Flat in 1988.

\begin{tabular}{|c|c|c|c|c|c|c|}
\hline \multirow[b]{2}{*}{ Species } & \multicolumn{2}{|c|}{$\begin{array}{l}\text { YUF016 } \\
\text { (455 m from GZ) }\end{array}$} & \multicolumn{2}{|c|}{$\begin{array}{c}\text { YUF017 } \\
(1060 \mathrm{~m} \text { from GZ) }\end{array}$} & \multicolumn{2}{|c|}{$\begin{array}{c}\text { YUF018 } \\
\text { (1591 } \mathrm{m} \text { from } \mathrm{GZ} \text { ) }\end{array}$} \\
\hline & $\% \mathrm{~T}$ & $\mathrm{M} / \mathrm{F}$ & $\% \mathrm{~T}$ & $\mathrm{M} / \mathrm{F}$ & $\% \mathrm{~T}$ & $M / F$ \\
\hline DIP MER & 94.2 & $1.1: 1$ & 87.9 & $1.6: 1$ & 37.5 & 1.1:1 \\
\hline DIP MIC & - & - & -- & - & 20.0 & $1.7: 1$ \\
\hline PER FOR & 2.9 & 1.0 & -- & - & $\cdots$ & - \\
\hline PER LON & - & - & $\cdots$ & - & 15.0 & $1: 1$ \\
\hline AMM LEU & 2.9 & $0: 1$ & 6.1 & ${ }^{*} 0: 1$ & 15.0 & $2: 1$ \\
\hline ONY TOR & - & - & 3.0 & $1: 0$ & 10.0 & $1: 3$ \\
\hline SYL AUD & - & - & 3.0 & indet & 2.5 & indet \\
\hline \multicolumn{7}{|c|}{$\begin{array}{l}\text { In the case of } S \text {. audubonii, only one juvenile of indeterminate sex was } \\
\text { captured. } \\
\text { "One animal of undetermined sex not included. }\end{array}$} \\
\hline
\end{tabular}

Dipodomys merriami in 1988 decreased as distance from GZ increased, while Dipodomys microps was present only at the farthest plot, $1591 \mathrm{~m}$ from $\mathrm{GZ}$, where the species diversity was the greatest. The total number of individual captures on all three plots was fairly low ( 34 animals at $455 \mathrm{~m}, 33$ at $1060 \mathrm{~m}$, and 40 at $1591 \mathrm{~m}$ ) and were not significantly different (chisquare, $p>0.05$ ). Animals were captured an average of 2.1 times at $455 \mathrm{~m}, 2.4$ times at 1060 $\mathrm{m}$, and 1.9 times at $1591 \mathrm{~m}$; the percent trap successes on the three plots were similar $(19.0 \%$, $20.6 \%$, and $19.3 \%$, respectively).

The ratio of males to females of the most commonly captured species did not differ significantly from 1:1 (Table 4.16). Mean weights of adult male Dipodomys merriami (Table 4B-2, Appendix 4B) were significantly heavier than adult females at $455 \mathrm{~m}$ (ANOVA, $\mathrm{p}=$ $0.028)$ and $1060 \mathrm{~m}(\mathrm{p}=0.002)$ but not significantly heavier at $1591 \mathrm{~m}(\mathrm{p}>0.05)$. There was no significant difference between the mean weights of adult females at the three distances or between the mean weights of adult males at the three sites (ANOVA, p>0.05). There was no significant difference between plots and combined weights of adult $D$. merriami. However, the combined mean weight of adult females from all three plots was significantly less than the combined mean weight of males $(p<0.001)$.

A comparison of 1962 pre- and post-test estimated densities (Table 4.17) to 1988 estimated values (Table 4.15) indicated a reinvasion closer to $G Z$ by Dipodomys merriami at numbers greater than were originally present ( $1.5 /$ ha pre-test to $23.7 /$ ha in 1988 at $455 \mathrm{~m}$ ). Other commonly captured species during pre-test sampling which were obliterated up to $1212 \mathrm{~m}$ 


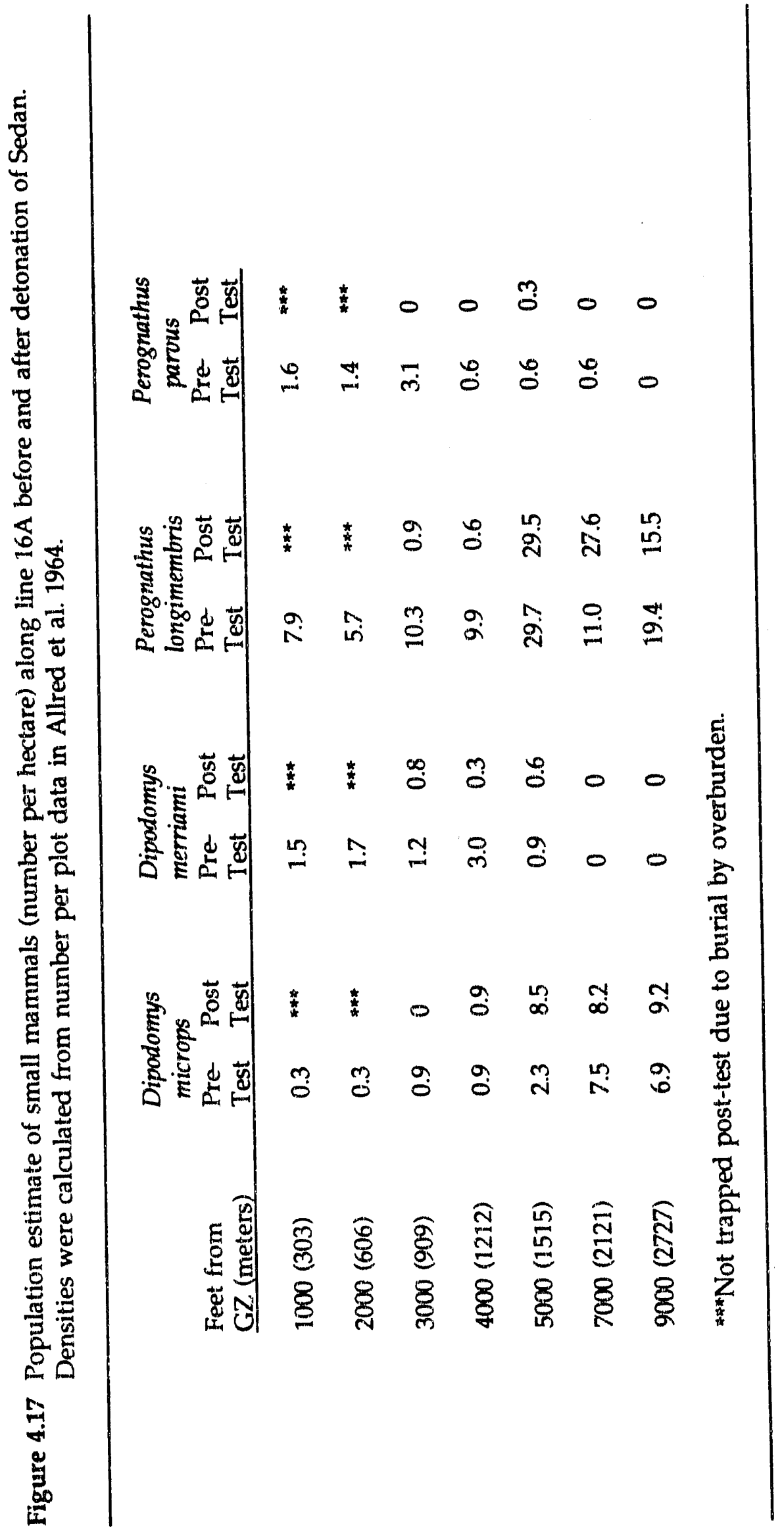


from GZ (Dipodomys microps and Perognathus parvus) have apparently not moved closer to GZ than post-test distances. In the case of $P$. parous, one animal was captured $915 \mathrm{~m}$ from $\mathrm{GZ}$ in 1983 during intensive trapping ( $O^{\prime}$ Farrell and Sauls 1987). Since then, this species has not been captured at the Sedan location.

Perognathus longimembris, present post-test at $909 \mathrm{~m}$ (3000 ft), was not captured in 1988 closer than $1591 \mathrm{~m}$ from GZ and in what appeared to be greatly reduced numbers (29.5/ha at 1515 $\mathrm{m}$ post-test versus $4.3 / \mathrm{ha}$ in 1988 at $1591 \mathrm{~m}$ ). Prior to the Sedan detonation, this area was used for a number of aboveground tests, so that the area was already disturbed. What might be occurring is that pre-test and post-test data are showing the continued dispersal of rodents (e.g., $P$. longiniembris) away from the disturbed areas, and into less disturbed habitat with a greater abundance of cover and food, with the exception of Dipodomys merriami, which does well in disturbed areas with minimal shrub cover.

\section{Disturbances from Nałurally Occurring Gopher Populations}

One site in Mercury Valley was censused on 21, 22, and 26 July 1988 for small mammals to determine species composition and estimated densities on an area denuded by gophers, Thomomys umbrinus. The vegetation consisted of a sparse cover of Stanleya pinnata (see Hunter, Section 1, p 20 [subject to change] of this report). An $8 \times 8$ plot (MER002) was established in the middle of the gopher area, and another $8 \times 8$ plot (MER003) was set up in an undisturbed Larrea-Ambrosia community $450 \mathrm{~m}$ to the northwest. Estimated densities of the most commonly captured species and presence of other species are shown in Table 4.18.

Table 4.18 Estimated densities (number/ha $\pm 2 \mathrm{SE}$ ) of small mammals on the BECAMP plots (1.44 ha) at a gopher site in Mercury Valley in August 1988. Numbers in parentheses are individuals captured.

\begin{tabular}{|c|c|c|c|c|}
\hline Species & \multicolumn{2}{|c|}{$\begin{array}{c}\text { MER002 } \\
\text { (Gopher area) }\end{array}$} & \multicolumn{2}{|l|}{$\begin{array}{r}\text { MER003 } \\
\text { (Control) }\end{array}$} \\
\hline DIP MER & $6.9 \pm 0$ & (10) & $10.5+0.6$ & (15) \\
\hline DIP MIC & $4.5 \pm 1.2$ & (6) & $6.4 \pm 0.8$ & (9) \\
\hline PER LON & $=$ & & $8.0 \pm 2.7$ & (10) \\
\hline PER FOR & -- & & $18.6 \pm 6.8$ & (20) \\
\hline ONY TOR & $*$ & (2) & $\bar{*}$ & (3) \\
\hline AMM LEU & $*$ & (2) & $*$ & (1) \\
\hline Total N/ha & 13.8 & (20) & 40.3 & $(58)$ \\
\hline Total species & 4 & & 6 & \\
\hline
\end{tabular}

* Species present but data insufficient to calculate density. 
Note that not one gopher was trapped in the gopher area. This in itself is not a significant indication of the absence of gophers. It is most likely due to the fact that fossorial gophers are rarely captured in Sherman traps of the type used. More notable was the absence of Perognathus spp. in the disturbed area.

Nearly three times as many individual animals were caught on the control plot (58 animals) as compared to the disturbed area (20 animals). Total captures were also greater on the control (101 total captures as compared to 41 total captures), as was percent trap success (26.3 and $10.7 \%$ ). Animals were captured an average of 2.1 times on the gopher area and 1.7 times on the control.

Table 4.19 Percent of total captured population (\% $\mathrm{T})$ and sex ratios $(\mathrm{M} / \mathrm{F}=$ male : female) of small mammals on the BECAMP plots at a gopher disturbed site in Mercury Valley on NTS in 1988.

\begin{tabular}{|c|c|c|c|c|}
\hline \multirow[b]{2}{*}{ Species } & \multicolumn{2}{|c|}{$\begin{array}{l}\text { MER002 } \\
\text { (Gopher area) }\end{array}$} & \multicolumn{2}{|c|}{$\begin{array}{r}\text { MER003 } \\
\text { (Control) }\end{array}$} \\
\hline & $\% \mathrm{~T}$ & $\mathrm{M} / \mathrm{F}$ & $\% \mathrm{~T}$ & $\mathrm{M} / \mathrm{F}$ \\
\hline DIP MER & 50.0 & $1: 1$ & 25.9 & $1.1: 1$ \\
\hline DIP MIC & 30.0 & $1: 2$ & 15.5 & $1.3: 1$ \\
\hline PER LON & $\ldots$ & - & 17.2 & $1: 1$ \\
\hline PER FOR & $\ldots$ & $\ldots$ & 34.5 & $1: 1.1$ \\
\hline ONY TOR & 10.0 & $1: 1$ & 5.2 & $2: 1$ \\
\hline AMM LEU & 10.0 & $1: 1$ & 1.7 & $1: 0$ \\
\hline
\end{tabular}

Of the animals captured on the gopher plot, $80.0 \%$ were kangaroo rats, compared to $41.4 \%$ on the control (Table 4.19). Approximately half, $51.7 \%$, of the animals on the control plot were Perognathus spp. The ratio of males to females for the most commonly captured species did not differ significantly from 1:1 (Table 4.19). There were no significant differences between mean weights of $D$. merriami males and females on the same plot or between adult males and adult females on different plots (Appendix 4B).

\section{TRAP DENSITY EXPERIMENT}

Results from the trap density experiments conducted on the Jackass Flats and Frenchman Flat baseline plots are summarized in Table 4.20. In both plots, the greatest number of new captures of Perognathus longimembris was on the $10 \times 10$ grids, 10 in Jackass Flats and 4 in Frenchman Flat, where the traps were only $7.5 \mathrm{~m}$ apart. A total of 21 new animals were captured on the Jackass Flats plot and 11 new animals were captured on the Frenchman Flat plot. The $10 \times 10$ plots also had the best percent success rate for recapture of $P$. longimembris (44\% on JAF001 and 57\% on FRF001). No more than 2 new Dipodomys merriami were 
Table 4.20 Number of animals per hectare and percent trap success during one day of trapping on $4 \times 4,5 \times 5,7 \times 7$, and $10 \times 10$ grids and three days of trapping on a $12 \times 12$ grid. Densities were calculated using actual grid size without any boundary.

Lackass Flats Density Experiment:

\begin{tabular}{|c|c|c|c|c|c|c|}
\hline & \multicolumn{6}{|c|}{ Traps Per Hectare } \\
\hline & $\begin{array}{c}4 \times 4 \\
50.6 / \mathrm{ha} \\
\end{array}$ & $\begin{array}{c}5 \times 5 \\
69.4 / \mathrm{ha} \\
\end{array}$ & $\begin{array}{c}7 \times 7 \\
118.9 / \mathrm{ha}\end{array}$ & $\begin{array}{l}10 \times 10 \\
219.5 / \text { ha }\end{array}$ & $\begin{array}{r}12 \times 1 \\
105.9 / \mathrm{ha} \\
\end{array}$ & Seber \\
\hline PER LON & 6.3 & 11.1 & 12.0 & 30.6 & 24.6 & 41.1 \\
\hline DIP MER & 9.5 & 11.1 & 21.8 & 17.6 & 12.1 & 12.6 \\
\hline Trap success & $25 \%$ & $28 \%$ & $26 \%$ & $23 \%$ & $18 \%$ & \\
\hline
\end{tabular}

Frenchman Flat Density Experiment:

\begin{tabular}{ccccccc} 
& \multicolumn{9}{c}{ Traps Per Hectare } \\
& $4 \times 4$ & $5 \times 5$ & $7 \times 7$ & $10 \times 10$ & \multicolumn{2}{c}{$12 \times 12$} \\
& $50.6 /$ ha & $69.4 /$ ha & $118.9 /$ ha & $219.5 /$ ha & $105.9 /$ ha & Seber \\
\cline { 2 - 7 } PER LON & 12.6 & 5.6 & 12.1 & 26.3 & 23.5 & 28.8 \\
DIP MER & 0.0 & 11.1 & 7.3 & 8.8 & 5.9 & 6.0 \\
Trap success & $25 \%$ & $2.8 \%$ & $16 \%$ & $16 \%$ & $17 \%$ & \\
\hline
\end{tabular}

captured on any of the grids. The highest percent success recapture of $D$. merriami for Jackass Flats occurred on the $10 \times 10$ grid ( 6 of 9, or $67 \%$ ), but the $7 \times 7$ grid percent success was only $3 \%$ lower $(7$ of 11 , or $64 \%$ ). $100 \%$ of the D. merriami on the Frenchman Flat $7 \times 7$ grid were recaptured ( 2 of 2 ) while 3 of 7 , or $43 \%$, were captured on the $10 \times 10$ grid.

These results seem to indicate that when sampling Perognathus longimembris, spacing between traps might be shorter, or the smaller home range should be considered when estimating their density; otherwise, relative abundance may be underestimated. These results suggest that any density comparisons between plots of differing trap distances should not be considered valid unless the trap difference is somehow accounted for in the estimation. However, using the original number of 67 Perognathus longimembris captured on the Jackass Flats plot (24.6/ha) and adding the number of new captures of 10 on the $10 \times 10$ plot $(21.9 / \mathrm{ha})$, the resultant 46.5 animals per hectare was in the range of the calculated Seber estimate (Table 4.2 , without $7.5 \mathrm{~m}$ boundary) of $41.1 \pm 13.6$ animals per hectare on JAF001, assuming that the $10 \times 10,0.56$-ha plot is representative of the total plot. P. longimembris 
captured on the FRF001 plot ( 32.3 animals per hectare) would also fall within the estimated density and $95 \%$ confidence interval given by the Seber estimate for that plot $(28.8 \pm 4.8)$. The Seber estimates of the standard errors for these two plots accurately predicted that over twice as many new $P$. longimembris remained uncaptured after the three trapping nights on the Jackass Flats plot $(13.6 / \mathrm{ha})$ as compared to the Frenchman Flat plot $(4.8 / \mathrm{ha})$.

Density of Perognathus longimembris did increase as trap density increased, but did not reach a plateau. Density of Dipodomys merriami on the Jackass Flats and Frenchman Flat plots also increased as trap density increased, but reached a plateau at the $7 \times 7$ plot in Jackass Flats and the $5 \times 5$ plot in Frenchman Flat. Further investigation is needed before any real conclusions can be made.

\section{SQUIRREL. TRAPPING}

The only squirrels captured during day-time trapping were white-tailed antelope squirrels, Ammospermophilus leucurus. One female was trapped on the Jackass Flats plot between 0800 and 1000 hours. Two males were captured on Yucca Flat between 1000 and 1200 hours and one female was caught between 1400 and 1600 hours. All of the squirrels captured were new and had not been caught during spring nocturnal mammal trapping.

During spring trapping, when the $8 \times 9 \times 30 \mathrm{~cm}$ Sherman traps were left open from late afternoon until just after sunrise of the next day, no squirrels were captured in Jackass Flats in 1988. In 1987, however, one A. lewcurus and one Spermophilus tereticaudus, round-tailed ground squirrel, were captured during nocturnal mammal trapping in July. During 1988 spring nocturnal mammal trapping on the Yucca Flat baseline monitoring site, four A. leucurus were captured while two were captured in July of 1987 . One S. tereticaudus was also captured on the Frenchman Flat baseline plot in July of 1987 during nocturnal mammal trapping.

It is evident that one day of trapping was not sufficient to adequately sample diurnal squirrels. Based on four $A$. leucurus captured in traps baited after 1730 hours on the Yucca Flat plot, as well as crepuscular observations by O'Farrell and Clark (1984), and bimodal daily activity patterns of other diurnal rodents (Drabek, 1973; Ilan and Yom-Tov, 1990) a longer sampling day may be necessary if the animals are active in the early morring as well as later afternoon when temperatures are cooler.

\section{RABBIT AND HARE SURVEYS}

Jackrabbits (Lepus californicus), desert cottontail rabbits (Sylvilagus audubonii), and Nuttall's cottontail rabbits (Sylvilagus nuttallii) were surveyed in 1988 on the BECAMP baseline monitoring plots in Jackass Flats between 2 June and 9 June, in Frenchman Flat between 13 June and 21 June, in Yucca Flat between 24 June and 30 June, and on Pahute Mesa between 24 June and 30 June. Transects were the same transects used to estimate lizard 
populations and were 2500 meters long. Desert cottontails were observed only on the Yucca Flat baseline plot, while Nuttall's cottontails were observed only on the Pahute Mesa plot. Jack rabbits were observed on all four of the baseline sites surveyed.

Densities and mean flushing distances are shown in Table 4.21. Fall jackrabbit density in

Table 4.21 Estimated densities ( $D=$ number/hectare $\pm 1 \mathrm{SE}$ ) and mean flushing distance $(F=$ meters $\pm 1 \mathrm{SE})$ of rabbits and hares on BECAMP baseline sites on NTS in June 1988.

\begin{tabular}{lcccccc}
\hline & \multicolumn{2}{c}{ SYL AUD } & \multicolumn{2}{c}{ SYL NUT } & \multicolumn{3}{c}{ LEP CAL } \\
Plot & $D$ & $F$ & $D$ & $F$ & $D$ & D \\
\hline & & & & & & \\
JAF001 & - & - & - & - & $0.11 \pm 0.19$ & $13.1 \pm 7.63$ \\
FRF001 & - & - & - & - & $0.11 \pm 0.14$ & $33.3 \pm 19.4$ \\
YUF001 & $0.14 \pm 0.38$ & $10.6 \pm 6.6$ & - & -- & $0.01 \pm 0.03$ & $27.3 \pm 27.3$ \\
PAM001 & -- & -- & $1.00 \pm 0.43$ & $4.9 \pm 3.7$ & $0.52 \pm 0.55$ & $9.5 \pm 10.1$ \\
\hline
\end{tabular}

northern Frenchman Flat in 1987 was estimated to be 0.10 / ha with a mean flushing distance of $33.5 \mathrm{~m}$. Sirnilarly, desert cottontail density was estimated at $0.23 /$ ha and mean flushing distance of $3.3 \mathrm{~m}$ (Hunter and Medica 1989).

Rabbits and hares were also surveyed on subsidiary plots in Yucca Flat (5, 6 July) and Mid Valley (7 June). Jackrabbit densities on the T1 blast site (YUF009) and control (YUF010) were estimated at $0.32 /$ ha and $0.66 / \mathrm{ha}$, respectively. Lengths of the transects were $1560 \mathrm{~m}$ each. Mean flushing distance on the denuded blast area was $16.7 \pm 15.0 \mathrm{~m}$ as compared to $2.4 \pm 2.4$ $m$ on the control. No cottontail rabbits were observed on either plot. No jackrabbits or cottontail rabbits were observed on the three plots on the eastern side of Yucca Flat (T3, 3B consolidation site, and control). This side of Yucca Flat has been used extensively for nuclear testing, which has left the area disturbed with little vegetative cover. Rabbits (Sylvilagus) are usually found in habitat that will provide shelter for concealing themselves from predators and a secluded nesting area or burrow for their altricial young. Hares (Lepus) will generally outrun a predator and therefore require less vegetation. Hares also do not construct burrows, and the precocial young do well in exposed and open habitats. (Zeveloff \& Collett 1988:84). No rabbits were observed on the Mid Valley burn area (MID002) or the control (MID003) during transect surveys. 


\section{DISCUSSION AND RECOMMENDATIONS}

\section{RARE SPECIES}

It is evident from the entire trapping record on the Nevada Test Site, as well as the differences between species captured during spring and summer trapping on the same plots in 1988, that intensive trapping, such as was done by BYU during 1959 to 1965 Jorgensen and Hayward 1965), is necessary to adequately assess the complete species composition of a particular area. Many species that are recorded for an area could erroneously be assumed to have disappeared, when in fact they are only present in very low numbers or during different seasons. Therefore, the probability of capture during a trapping duration of only three days is low. For the purpose of assessing the population density of the dominant species, however, a short trapping duration appears to be adequate. To adequately determine whether or not a particular species has disappeared from a disturbed area, more intensive trapping should be undertaken.

\section{TRAP SUCCESS}

The average capture rates of animals on the BECAMP plots (total number of captures/ number of different animals) are listed in Table 4.22. Average captures consistently showed that the control animals were caught less frequently than the animals on the corresponding disturbed plot. That is, individual animals on the disturbed plots tended to be captured at least two out of the three nights, while individual animals on the control plots were captured less than two out of the three nights. The combined average capture of all disturbed plots (2.1) was significantly greater than the combined average capture rate of all control plots (1.8) (t-test, $\mathrm{p}<0.001$ ).

The combined average percent trap success (number of total captures/trap nights), however, was greater on the controls (21.0) than on the disturbed sites (19.4) but not significantly greater. These two 'rapping parameters indicate that a greater number of individual animals and species of animals (Table 4.22) are being captured on the control areas. Since two traps were activated at each trapping station, and it was not common to capture animals in both traps, "trap happy" rodents probably did not decrease the chances of catching other unmarked animals on the disturbed areas. Therefore, it can be assumed that the number of animals that were likely to be trapped were captured, and that the density estimates adequately describe the animal population at a particular site.

\section{TYPE OF DISTURBANCE RELATED DIFFERENCES}

In all of the BECAMP paired plots sampled in 1988, with the exception of the Rock Valley plots, disturbed areas had either no Dipodomys microps present or present at numbers much lower than the control plots. Areas disturbed by blast effects from nuclear detonation had no $D$. microps while areas disturbed by burning, blading and gophers still showed the presence of $D$. microps but at lower numbers. The Rock Valley enclosed plot had a D. microps 
Table 4.22 Number of species, average capture rates, and percent trap success on baseline, disturbed, and control monitoring plots in 1988 on NTS.

\begin{tabular}{|c|c|c|c|c|c|}
\hline Plot & & $\begin{array}{l}\text { Type of } \\
\text { Disturbance }\end{array}$ & $\begin{array}{l}\text { umber of } \\
\text { pecies }\end{array}$ & $\begin{array}{l}\text { Average } \\
\text { Capture }\end{array}$ & $\begin{array}{l}\text { Percent } \\
\text { Success } \\
\end{array}$ \\
\hline FRF001 & & Baseline & 4 & 1.8 & 16.9 \\
\hline JAF001 & & Baseline & 3 & 1.6 & 18.4 \\
\hline YUF001 & & Baseline & 6 & 2.0 & 22.2 \\
\hline PAM001 & & Baseline & 7 & i.8 & 11.3 \\
\hline RAM001 & & Baseline & 6 & 1.6 & 6.6 \\
\hline MIDO02 & & Burn & 6 & 1.9 & 10.9 \\
\hline $\mathrm{MID} 003$ & & Control & 8 & 1.6 & 10.7 \\
\hline \multirow[t]{3}{*}{ RED001 } & (July) & Burn & 7 & 2.1 & 22.9 \\
\hline & (August) & & 7 & 1.6 & 33.9 \\
\hline & (October) & & 3 & 2.4 & 24.8 \\
\hline \multirow[t]{3}{*}{ RED002 } & (July) & Control & 7 & 1.8 & 25.0 \\
\hline & (August) & & 6 & 1.6 & 25.0 \\
\hline & (October) & & 7 & 2.2 & 31.4 \\
\hline ROV008 & & Fenced & 6 & 2.3 & 30.9 \\
\hline ROV007 & & Control & 8 & 2.0 & 24.5 \\
\hline YUF009 & & Aboveground blast & 6 & 2.5 & 19.4 \\
\hline YUF010 & & Control & 6 & 1.9 & 26.0 \\
\hline YUF011 & & Blading & 3 & 2.3 & 6.0 \\
\hline YUF012 & & Control & 6 & 1.8 & 22.1 \\
\hline YUF013 & & Aboveground blast & 3 & 2.3 & 8.6 \\
\hline YUF016 & & Sedan throw-out, $455 \mathrm{~m} \mathrm{GZ}$ & 3 & 2.1 & 19.0 \\
\hline YUF017 & & Sedan throw-out, $1060 \mathrm{~m} \mathrm{GZ}$ & Z 4 & 2.4 & 26.6 \\
\hline YUF018 & & Sedan control, $1591 \mathrm{~m} \mathrm{GZ}$ & 6 & 1.9 & 19.3 \\
\hline MER002 & & Gopher denuded & 4 & 2.1 & 10.7 \\
\hline MER003 & & Control & 6 & 1.7 & 26.3 \\
\hline
\end{tabular}

population of over twice that of the unfenced plot. The enclosed area has an abundant shrub cover available for food supply aru cover. The absence of perennial shrub cover on the other disturbed sites may be limiting the $D$. microps populations.

Dipodomys merriami was generally the dominant species on the disturbed plots (again, with the exception of Rock Valley) while on the control, D. merriami was usually co-dominant with 
Perognathus longimembris. Bladed, blasted, and gopher denuded areas had two to three fewer species than the corresponding control, except for the T1 blast area, while the areas disturbed due to burning had one to two fewer species than the control (Table 4.22).

Each of the Yucca Flat control plots, as well as the baseline site, had six different species trapped. Five of the six were the same: Dipodomys merriami, Dipodomys microps, Perognathus longimembris, Onychomys torridus, and Ammospermophilus leucurus. The sixth species was either Sylvilagus audubonii, Peromyscus maniculatus or Reithrodontomys megalotis, of which only one or two animals were trapped.

\section{FUTURE MONITORING PLAN}

Fluctuations in population sizes are a normal occurrence in some small mammals (Dipodomys spp. and Perognathus spp.), while other small rodents (e.g. Peromyscus spp. and Onychomys spp.) occur at low densities that are relatively stable from year to year (Brown and Heske 1990; Swihart and Slade 1990; Drost and Fellers 1991). Annual cycles in natural communities have been attributed to both abiotic (climate, rainfall) and biotic (food abundance, density related stress, predators) factors (Hansson 1987; Lidicker 1988). The presence of and interaction with other rodent species (Brown and Heske 1990; Swihart and Slade 1990) and breeding suppression in peak periods (Terman 1965; Van Horne 1981; Drost and Fellers 1991) are density-related changes in the reproductive effort and success in several rodent species. Monitoring on the Yucca Flat baseline site will continue on a yearly basis in order to docurnent any natural changes in the small mammal community at this site. Other baseline sites will be monitored on a three to four year cycle, while subsidiary sites will be trapped at three year intervals. In this way, it is hoped to see what is happening on the NTS in regards to testing activity and what is occurring due to the forces of nature.

In future years, the biological effects of additional disturbances (i.e., cratering as a result of underground nuclear testing, roadside effects, alpha-contaminated areas) and replication of blast effects at additional ground-zero sites and burned areas will be studied. In addition, it is hoped to follow the progress of the contaminated waste dumps that have been cleaned up and revegetated. 


\section{REFERENCES}

Allred, D. M. and D E. Beck. 1963. Comparative ecological studies of animals at the Nevada Test Site. In: Radioecology. V. Schultz and A.W. Klement, Jr., eds. Reinhold Publishing Corp., New York, NY and The American Institute of Biological Sxiences, Washington, D.C. pp. 327-331.

Allred, D. M., D E. Beck, and C. D. Jorgensen. 1963. Biotic Communities of the Nevada Test Site. Brigham Young University Sci. Bull., Biol. Ser. 2(2):1-52.

Allred, D. M., D E. Beck, and C. D. Jorgensen. 1964. Close-in effects of an urderground nuclear detonation on small mammals and selected invertebrates. USAEC Report, PNE226F IV. 22pp.

Beatley, J. C. 1969. Dependence of desert rodents on winter annuals and precipitation. Ecology 50(4):405-425.

- 1976a. Environments of kangaroo rats (Dipodomys) and effects of environmental change in populations in southern Nevada. J. Mammal. 57(1):67-93.

1976b. Rainfall and fluctuating plant populations in relation to distribution and numbers of rodents in southem Nevada. Oexologica (Berlin) 24:21-42.

Bradley, W. G. and R. A. Mauer. 1971. Reproduction and food habits of Merriam's kangaroo rat, Dipodomys merriami. J. Mammal. 52(3):497-507.

Brown, J. H. and E. J. Heske. 1990. Temporal changes in a Chihuahuan Desert rodent community. Oikos. 59:290-302.

Drabek, C. U. 1973. Home range and daily activity of the round-tailed ground squirrel, Spermophilus tereticaudus neglectus. Amer. Midl. Natur. 89(2):287-293.

Drost, C. A. and G. M. Fellers. 1991. Density cycles in an island population of deer mice, Peromyscus maniculatus. Oikos. 60:351-364.

French, N. R., B. G. Maza, and A. P. Aschwanden. 1967. Life spans of Dipodomys and Perognathus in the Mojave Desert. J. Mammai. 52(3):497-507.

French, N. R., B. G. Maza, H. O. Hill, A. P. Aschwanden, and H. W. Kaaz. 1974. A population study of irradiated desert rodents. Ecol. Monographs 44(1):45-72. 
Hansson, L. 1987. An interpretation of rodent dynamics as due to trophic interactions.

Oikos. 50:308-318

Hunter, R. B. and P. A. Medica. 1989. Status of the flora and fauna of the Nevada Test Site: results of continuing basic environmental research. DOE/NV/10630-2. 103pp.

Ilian, M. and X. Yom-Tov. 1990. Diel activity pattern of a diumal desert rodent, Psammomys obesus. J. Mammal. 71(1):66-69.

Jorgensen, C. D. and C. L. Hayward. 1965. Mammals of the Nevada Test Site. Brigham Young University Sci. Bull., Biol., Ser. 6(3):1-81.

Jorgensen, C. D., H. D. Smith and J. R. Garcia. 1980. Temporal activity patterns of a Dipodomys ordii population. Great Basin Nat. 40(3):282-286.

Kenagy, G. J. 1973. Daily and seasonal patterns of activity and energetics in a heteromyid rodent community. Ecology 53:1201-1219.

Lidicker, W. Z. Jr. 1988. Solving the enigma of nicrotine "cycles". J. Mammal. 69:225-235.

Maza, B. G., N. R. French and A. P. Aschwanden. 1973. Home range in a population of Heteromyid rodents. J. Mammal. 54:405-425.

Medica, P. A. 1990. Noteworthy mammal distribution records for the Nevada Test Site. Great Basin Nat. 50(1):83-84.

O'Farrell, M. J. and W. A. Clark. 1984. Notes on the white-tailed antelope squirrel, Ammospermophilus leucurus, and the pinyon mouse, Peromyscus truet, in north central Nevada. Great Basin Nat. 44(3):428-429.

O'Farrell, T. P. and M. L. Sauls. 1987. Small mammals inhabiting nuclear-event sites: population characteristics and radionuclide body burden. In: The Dynamics of Transuranics and Other Radionuclides in Natural Environments. NVO-272. pp 279-301.

Seber, G. A. F. 1982. The Estimation of Animal Abundance and Related Parameters. New York, NY: Macmillan Publishing Co, Inc. Second ed.

Swihart, R. K. and N. A. Slade. 1990. Long-term dymamics of an eariy successional small mammal community. Am. Midl. Nat. 123:372-382.

Terman, C. R. 1965. A study of population growth and control exhibited in the lab by prairie deer mice. Ecology 46:890-895. 
Turner, F. B. (Coordinator) 1973. Rock Valley Validation Site Report. U.S./IBP Desert Biome Reports of 1972 Progress, 'Vertebrates'. Research Memo. RM 73-2:168-200.

Turner, F. B. (Coordinator). 1974. Rock Valley Validation Site Report. U.S./IBP Desert Biome. Reports of 1973 Progress. Research Memo RM 24-2:55-61.

Van De Graaf, K. M. and R. P. Balda. 1973. Importance of green vegetation for reproduction in the kangaroo rat Dipodomys merriami. J. Mammal. 54:509-512.

Van Horne, B. 1981. Demography of Peromyscus maniculatus populations in seral stages of coastal coniferous forest in southeast Alaska. Can. J. Zool. 59:1045-1061.

White, E. D. and D. M. Allred. 1961. Range of kangaroo rats in areas affected by atomic detonations. Utah Acad. Sci., Arts and Letters 38:101-110.

Whitford, W. G. 1973. Jornada Validation Site Report. U.S./IBP Desert Biome. Reports of 1972 Progress. RM 73-4:135-167.

Whitford, W. G. 1976. Temporal fluctuations in density and diversity of desert rodent populations. J. Mammal. 57:351-369.

Zeveloff, S. I. and F. R. Collett. 1988. Mammals of the Intermountain West. Salt Lake City, UT: University of Utah Press. 365pp. 


\section{Appendix 4A}

\section{Species and Number Captured During Three Days of Spring}

Sampling and One Day of Summer Sampling on Five Baseline

Monitoring Plots on the NTS in 1988

Plot

\begin{tabular}{|c|c|c|c|c|c|c|c|c|c|}
\hline \multirow[b]{2}{*}{ Species } & \multicolumn{2}{|c|}{ JAF001 } & \multicolumn{2}{|c|}{ FRF001 } & \multicolumn{2}{|c|}{ YUF001 } & \multicolumn{2}{|c|}{ PAM001 } & \multirow{2}{*}{$\begin{array}{l}\text { RAM001 } \\
\text { SU }\end{array}$} \\
\hline & SP & SU & SP & SU & SP & SU & SP & $\mathrm{SU}$ & \\
\hline AMM LEU & - & - & 1 & 1 & 4 & - & 1 & - & - \\
\hline DIP DES & 1 & - & - & - & - & - & - & - & - \\
\hline DIP MER & 33 & 31 & 16 & 18 & 17 & 14 & - & - & - \\
\hline DIP MIC & - & - & - & - & 16 & 11 & 2 & - & - \\
\hline MIC MEG & - & - & - & - & - & - & 1 & 2 & - \\
\hline NEO LEP & - & - & - & - & - & - & 2 & - & - \\
\hline ONY TOR & - & - & 1 & - & 2 & 4 & - & - & - \\
\hline PER LON & 67 & 23 & 64 & 29 & 57 & 6 & - & - & - \\
\hline PER PAR & - & - & - & - & - & - & 34 & 20 & 1 \\
\hline PER SPP & - & - & - & - & - & - & 13 & 6 & 16 \\
\hline PER TRU & - & - & - & - & - & - & - & - & 5 \\
\hline REI MEG & - & - & - & - & - & 1 & - & - & 2 \\
\hline SYL AUD & - & - & - & - & 1 & - & - & - & - \\
\hline SYL NUT & - & - & - & - & - & - & 1 & - & 4 \\
\hline TAM DOR & - & - & - & - & . & - & - & - & 9 \\
\hline
\end{tabular}




\section{Appendix 4B}

\section{Mean Weights of the Most Commonly Captured Species Found}

on BECAMP Monitoring Plots on the NTS in 1988

Table 4B-1 Adult summer mean weights (grams $\pm 2 \mathrm{SE}$ ) by sex of common rodent species on three BECAMP baseline monitoring plots in 1988.

\begin{tabular}{llllllll}
\hline & & \multicolumn{2}{c}{ JAF001 } & \multicolumn{2}{c}{ FRF001 } & \multicolumn{2}{c}{ YUF001 } \\
Species & Sex & N & Weight & N & Weight & N & Weight \\
PER LON & M & 11 & $8.7 \pm 0.7$ & 13 & $7.7 \pm 0.6$ & 2 & $8.5 \pm 3.0$ \\
& F & 11 & $8.8 \pm 0.8$ & 12 & $8.3 \pm 0.9$ & 4 & $8.0 \pm 0.8$ \\
DIP MER & M & 18 & $38.9 \pm 2.2$ & 5 & $38.6 \pm 5.3$ & 4 & $39.5 \pm 2.4$ \\
& F & 8 & $35.1 \pm 3.2$ & 5 & $37.0 \pm 2.8$ & 6 & $39.0 \pm 2.9$ \\
DIP MIC & M & 0 & - & 0 & - & 3 & $54.7 \pm 3.3$ \\
& F & 0 & - & 0 & - & 5 & $54.6 \pm 5.5$ \\
\hline
\end{tabular}




\section{Appendix 4B (Continued)}

Table 4B-2 Mean weights (grams $\pm 2 \mathrm{SE}$ ) by sex and age of Dipodomys merriami captured on BECAMP subsidiary plots on NTS in 1988. Numbers in parentheses are individuals weighed.

\begin{tabular}{|c|c|c|c|c|c|c|c|c|}
\hline Plot & $\begin{array}{l}\text { Male } \\
\text { Adult } \\
\end{array}$ & & $\begin{array}{l}\text { Female } \\
\text { Adult }\end{array}$ & & $\begin{array}{c}\text { Male } \\
\text { Juvenile }\end{array}$ & & $\begin{array}{c}\text { Female } \\
\text { Juvenile }\end{array}$ & \\
\hline $\begin{array}{l}\text { YUF009 } \\
\text { (May) } \\
\text { YUF009 }\end{array}$ & $44.1 \pm 1.5$ & (16) & $42.6 \pm 1.9$ & (9) & 18.0 & (1) & 26.0 & (1) \\
\hline $\begin{array}{l}\text { (August) } \\
\text { YUF010 }\end{array}$ & $42.6 \pm 3.2$ & (5) & $37.3 \pm 2.6$ & (4) & 30.0 & (1) & $8.5 \pm 1.0$ & (2) \\
\hline $\begin{array}{l}\text { (May) } \\
\text { YUF010 }\end{array}$ & $43.3 \pm 1.6$ & (11) & $42.1 \pm 2.4$ & (4) & & & $23.5 \pm 1.0$ & (2) \\
\hline (August) & $42.6 \pm 3.2$ & (5) & $37.3 \pm 2.6$ & (4) & 30.0 & (1) & $28.5 \pm 1.0$ & (2) \\
\hline YUF011 & $45.7 \pm 0.7$ & (4) & $42.8 \pm 2.6$ & (4) & & & & \\
\hline YUF012 & $45.0 \pm 2.3$ & (8) & $48.2 \pm 6.0$ & (3) & 22.0 & (1) & $21.6 \pm 1.6$ & (5) \\
\hline YUF013 & $41.3 \pm 1.3$ & (8) & $41.8 \pm 4.9$ & (4) & & & $32 . \overline{0}$ & (1) \\
\hline YUF016 & $43.3 \pm 2.0$ & (14) & $39.6 \pm 2.3$ & (9) & $25.7 \pm 2.7$ & (3) & $29.3 \pm 4.9$ & (6) \\
\hline YUF017 & $43.6 \pm 2.2$ & $(12)$ & $37.8 \pm 1.3$ & (7) & $30.9 \pm 2.2$ & (6) & $28.7 \pm 0.2$ & (4) \\
\hline YUF018 & $42.8 \pm 1.8$ & (5) & $38.9 \pm 3.3$ & (5) & $29.5 \pm 1.1$ & (3) & $27.5 \pm 7.0$ & (2) \\
\hline $\begin{array}{l}\mathrm{MIDOO2} \\
\mathrm{MIDO03}\end{array}$ & $\begin{array}{l}43.6 \pm 3.2 \\
41.0 \pm 2.0\end{array}$ & $\begin{array}{l}(8) \\
(3)\end{array}$ & $\begin{array}{l}44.1 \pm 3.7 \\
38.7 \pm 6.7\end{array}$ & $\begin{array}{l}(4) \\
(2)\end{array}$ & $27.1 \pm 2.3$ & (3) & & \\
\hline $\begin{array}{l}\text { RED001 } \\
\text { (July) } \\
\text { RED001 }\end{array}$ & $41.3 \pm 3.3$ & (8) & $39.3 \pm 3.2$ & (5) & $29.2 \pm 3.0$ & (6) & $26.3 \pm 3.3$ & (5) \\
\hline $\begin{array}{l}\text { (August) } \\
\text { REDO01 }\end{array}$ & $43.4 \pm 1.9$ & (13) & $39.4 \pm 2.6$ & (12) & $34.4 \pm 2.3$ & (4) & $32.3 \pm 3.2$ & (5) \\
\hline (October) & $39.1 \pm 1.2$ & (18) & $36.6 \pm 1.0$ & (13) & & & $31.9 \pm 1.0$ & (3) \\
\hline $\begin{array}{l}\text { REDO02 } \\
\text { (July) } \\
\text { RED002 }\end{array}$ & $40.0 \pm 3.2$ & (11) & $43.1 \pm 1.9$ & (10) & $27.3 \pm 1.7$ & (7) & $26.9 \pm 3.0$ & (3) \\
\hline $\begin{array}{l}\text { (August) } \\
\text { RED002. }\end{array}$ & $42.2 \pm 2.6$ & $(10)$ & $43.7 \pm 2.2$ & (11) & $31.4 \pm 4.2$ & (4) & $34.0 \pm 2.0$ & (2) \\
\hline (October) & $38.2 \pm 1.7$ & (17) & $37.1 \pm 1.5$ & (12) & 34.0 & (1) & $32.9 \pm 3.2$ & (2) \\
\hline $\begin{array}{l}\text { ROV002 } \\
\text { ROV007 }\end{array}$ & $\begin{array}{l}42.2 \pm 3.8 \\
41.0 \pm 1.4\end{array}$ & $\begin{array}{l}(3) \\
(8)\end{array}$ & $\begin{array}{l}46.8 \pm 1.5 \\
37.3 \pm 1.9\end{array}$ & $\begin{array}{l}(2) \\
(5)\end{array}$ & 25.0 & (I) & 29.5 & (1) \\
\hline $\begin{array}{l}\text { MER002 } \\
\text { MER003 }\end{array}$ & $\begin{array}{l}40.9 \pm 7.8 \\
39.6 \pm 3.3\end{array}$ & $\begin{array}{l}(4) \\
(7)\end{array}$ & $\begin{array}{l}40.5 \pm 5.4 \\
38.3 \pm 4.0\end{array}$ & $\begin{array}{l}(4) \\
(4)\end{array}$ & $\begin{array}{l}30.5 \\
25.3\end{array}$ & $\begin{array}{l}(1) \\
(1)\end{array}$ & $\begin{array}{c}32.3 \\
29.7 \pm 2.9\end{array}$ & $\begin{array}{l}\text { (1) } \\
\text { (3) }\end{array}$ \\
\hline
\end{tabular}




\section{Appendix 4B (Continued)}

Table 4B-3 Mean weight (grams \pm 2 SE) of Dipodomys microps on BECAMP subsidiary plots on NTS in 1988. Numbers in parentheses are individuals weighed.

\begin{tabular}{|c|c|c|c|c|c|c|c|c|}
\hline Plot & $\begin{array}{l}\text { Male } \\
\text { Adult }\end{array}$ & & $\begin{array}{c}\text { Female } \\
\text { Adult }\end{array}$ & & $\begin{array}{c}\text { Male } \\
\text { Juvenile }\end{array}$ & & $\begin{array}{l}\text { Female } \\
\text { Juvenile }\end{array}$ & \\
\hline $\begin{array}{l}\text { YUF010 } \\
\text { (May) } \\
\text { YUF010 } \\
\text { (August) }\end{array}$ & $\begin{array}{l}69.7 \pm 6.6 \\
54.7+3.3\end{array}$ & (4) & $\begin{array}{c}47.7 \\
54.6+5.5\end{array}$ & $\begin{array}{l}\text { (1) } \\
\text { (5) }\end{array}$ & 48.0 & (1) & 49.0 & (1) \\
\hline $\begin{array}{l}\text { YUF011 } \\
\text { YUF012 }\end{array}$ & $\begin{array}{c}58.0 \\
67.3 \pm 11.5\end{array}$ & $\begin{array}{l}\text { (1) } \\
\text { (2) }\end{array}$ & $52.0 \pm 7.2$ & (3) & & & 37.0 & (1) \\
\hline YUF018 & $58.0 \pm 9.4$ & (3) & $64.0 \pm 0.0$ & (2) & $44.0 \pm 2.0$ & (2) & 45.0 & (1) \\
\hline $\begin{array}{l}\text { MIDO02 } \\
\text { MID003 }\end{array}$ & 67.5 & (1) & 64.0 & (1) & $\begin{array}{l}44.5 \\
28.5\end{array}$ & $\begin{array}{l}(1) \\
(1)\end{array}$ & $37.2 \pm 8.4$ & (3) \\
\hline $\begin{array}{l}\text { RED001 } \\
\text { (July) } \\
\text { RED001 } \\
\text { (August) } \\
\text { RED001 } \\
\text { (October) }\end{array}$ & $\begin{array}{l}52.2 \pm 5.0 \\
56.5 \pm 11.2 \\
61.9 \pm 4.9\end{array}$ & (5) & $\begin{array}{c}51.3 \\
51.3 \pm 8.7 \\
54.6 \pm 7.4\end{array}$ & $\begin{array}{l}\text { (1) } \\
\text { (6) } \\
\text { (3) }\end{array}$ & & & 48.0 & (1) \\
\hline $\begin{array}{l}\text { RED002 } \\
\text { (July) } \\
\text { REDO02 } \\
\text { (August) } \\
\text { REDO02 } \\
\text { (October) }\end{array}$ & $\begin{array}{l}58.4 \pm 8.2 \\
61.4 \pm 3.9 \\
60.7 \pm 3.9\end{array}$ & $\begin{array}{r}(5) \\
(4) \\
(11)\end{array}$ & $\begin{array}{l}54.3 \pm 9.3 \\
59.0 \pm 8.4 \\
55.3 \pm 3.6\end{array}$ & $\begin{array}{l}(3) \\
(5) \\
(8)\end{array}$ & & & & \\
\hline $\begin{array}{l}\text { ROV002 } \\
\text { ROV007 }\end{array}$ & $\begin{array}{l}57.3 \pm 1.9 \\
59.0 \pm 3.7\end{array}$ & $\begin{array}{l}(30) \\
(12)\end{array}$ & $\begin{array}{l}53.4 \pm 1.5 \\
55.2 \pm 3.6\end{array}$ & $\begin{array}{l}(28) \\
(11)\end{array}$ & $\begin{array}{l}41.1 \pm 3.6 \\
44.0 \pm 3.6\end{array}$ & $\begin{array}{r}(11) \\
(7)\end{array}$ & $\begin{array}{l}41.4 \pm 2.1 \\
43.3 \pm 4.0\end{array}$ & $\begin{array}{r}(16) \\
(8)\end{array}$ \\
\hline $\begin{array}{l}\text { MER002 } \\
\text { MER003 }\end{array}$ & $\begin{array}{l}54.8 \pm 3.7 \\
55.8 \pm 5.4\end{array}$ & $\begin{array}{l}(2) \\
(4)\end{array}$ & $\begin{array}{l}61.2 \pm 9.7 \\
56.2 \pm 4.6\end{array}$ & $\begin{array}{l}\text { (3) } \\
\text { (3) }\end{array}$ & $\begin{array}{l}46.0 \\
47.0\end{array}$ & $\begin{array}{l}(1) \\
(1)\end{array}$ & 40.5 & (1) \\
\hline
\end{tabular}




\section{Appendix 4B (Continued)}

Table 4.B-4 Mean weight (grams \pm 2 SE) of Perognathus longimembris on BECAMP subsidiary plots on NTS in 1988. Numbers in parentheses are individuals weighed.

\begin{tabular}{|c|c|c|c|c|c|c|c|c|}
\hline Plot & $\begin{array}{l}\text { Male } \\
\text { Adult } \\
\end{array}$ & & $\begin{array}{l}\text { Female } \\
\text { Adult }\end{array}$ & & $\begin{array}{c}\text { Male } \\
\text { Juvenile }\end{array}$ & & $\begin{array}{c}\text { Female } \\
\text { Juvenile }\end{array}$ & \\
\hline YUF009 & $7.3 \pm 0.5$ & (5) & $7.6 \pm 0.5$ & (2) & & & & \\
\hline $\begin{array}{l}\text { YUF010 } \\
\text { (May) } \\
\text { YUF010 } \\
\text { (August) }\end{array}$ & $\begin{array}{c}7.7 \pm 0.3 \\
8.0\end{array}$ & $\begin{array}{r}\text { (17) } \\
\text { (1) }\end{array}$ & $\begin{array}{l}8.1 \pm 0.4 \\
7.1 \pm 1.0\end{array}$ & $\begin{array}{r}(21) \\
(4)\end{array}$ & & & & \\
\hline $\begin{array}{l}\text { YUF011 } \\
\text { YUF012 }\end{array}$ & $8.3 \pm 0.4$ & $(7)$ & $\begin{array}{l}8.0 \pm 0.0 \\
9.5 \pm 0.9\end{array}$ & $\begin{array}{r}(2) \\
(11)\end{array}$ & 7.0 & (1) & & \\
\hline YUF018 & $8.2 \pm 0.9$ & (3) & $7.8 \pm 1.7$ & (2) & & & 7.0 & (1) \\
\hline $\begin{array}{l}\mathrm{MIDO02} \\
\mathrm{MIDO03}\end{array}$ & $\begin{array}{l}8.2 \pm 2.0 \\
8.3 \pm 0.7\end{array}$ & $\begin{array}{l}(3) \\
(3)\end{array}$ & $\begin{array}{c}10.0 \pm 3.1 \\
9.5 \pm 1.0\end{array}$ & $\begin{array}{l}\text { (3) } \\
\text { (2) }\end{array}$ & & & 6.0 & (1) \\
\hline $\begin{array}{l}\text { RED001 } \\
\text { (July) } \\
\text { RED001 } \\
\text { (August) }\end{array}$ & $8.0 \pm 1.2$ & (3) & $\begin{array}{c}6.5 \pm 1.0 \\
8.0\end{array}$ & $\begin{array}{l}(2) \\
(1)\end{array}$ & & & & \\
\hline $\begin{array}{l}\text { REDO02 } \\
\text { (July) } \\
\text { REDO02 } \\
\text { (August) }\end{array}$ & 7.0 & (1) & $\begin{array}{c}8.0 \pm 1.2 \\
8.0\end{array}$ & $\begin{array}{l}\text { (3) } \\
\text { (1) }\end{array}$ & $6.5 \pm 1.0$ & (2) & & \\
\hline ROV007 & $8.0 \pm 0.3$ & (18) & $8.3 \pm 0.5$ & (12) & 5.0 & (1) & $5.5 \pm 1.0$ & (2) \\
\hline MER003 & $6.7 \pm 0.7$ & (3) & $7.4 \pm 1.0$ & (5) & $5.5 \pm 1.0$ & (2) & & \\
\hline
\end{tabular}




\section{Appendix 4C}

\section{Results of Density and Standard Error}

\section{Calculations Using Seber (1982:138)}

$\mathrm{N} 1$ = Total individuals marked before present trap night.

$\mathrm{XB}=$ New individuals captured during present trap night.

M2 = Number in N2 which were recaptured during present trap night.

$\mathrm{N} 2$ = Total of individuals captured during present trap night.

$\mathrm{N}^{*} /$ hectare $=$ Estimated number of animals per hectare.

$\mathrm{V} / \mathrm{Ha}^{\wedge} 2=$ Estimated variance per hectare squared for $\mathrm{N}^{*} / \mathrm{Ha}$.

$2 \mathrm{SE} / \mathrm{ha}=$ two times the estimated standard error per hectare for $\mathrm{N}^{*} /$ ha.

HFRF188SPRDEN

0

$\begin{array}{llllll}12-A P R-88 & 2 & 13-A P R-88 & 3 & 14-A P R-88\end{array}$

1 DIP MER

$2 N 1$

$3 \mathrm{XBB}$

$4 \mathrm{M} 2$

$5 \mathrm{~N} 2$

$6 \mathrm{N*} / \mathrm{HECTARE}$

$7 \mathrm{~V} / \mathrm{HA}^{\wedge} 2$

$82 \mathrm{SE} / \mathrm{HA}$

9

10 PER LON

$11 \mathrm{~N} 1$

$12 \mathrm{XB}$

$13 \times 2$

$14 \mathrm{~N} 2$

$15 \mathrm{N*} /$ HECTARE

$16 \mathrm{~V} / \mathrm{HA}^{-2} 2$

172 SE/HA

14

2

12

14

5

0.04

0.38

26

22

10

32

24.69

20.58

9.07
16

0

14

14

4.94

0

0

48

16

26

42

23.78

3.46

3.72 
Appendix 4C (Continued)

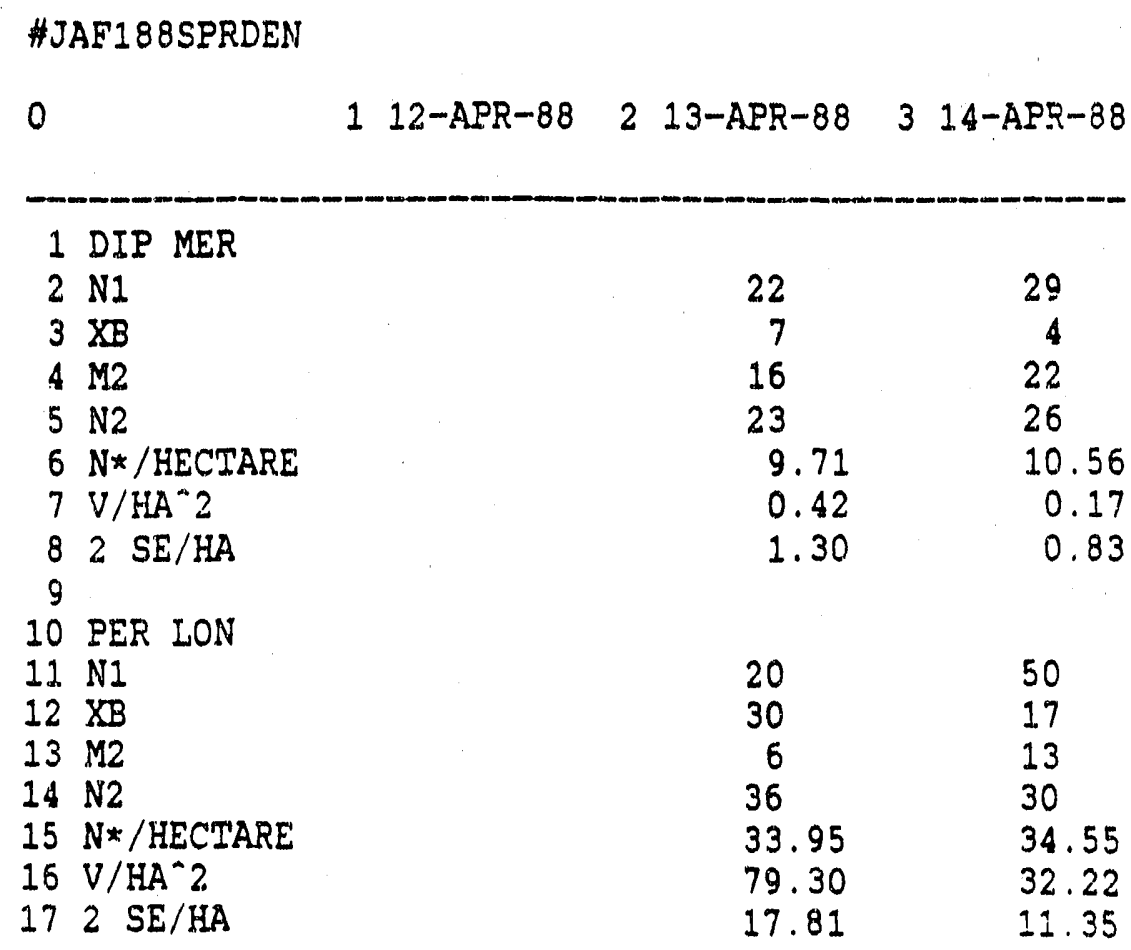

\#MER288SUMDEN

0

$121-A U G-88 \quad 2 \quad 22-A U G-88 \quad 3 \quad 26-A U G-88$

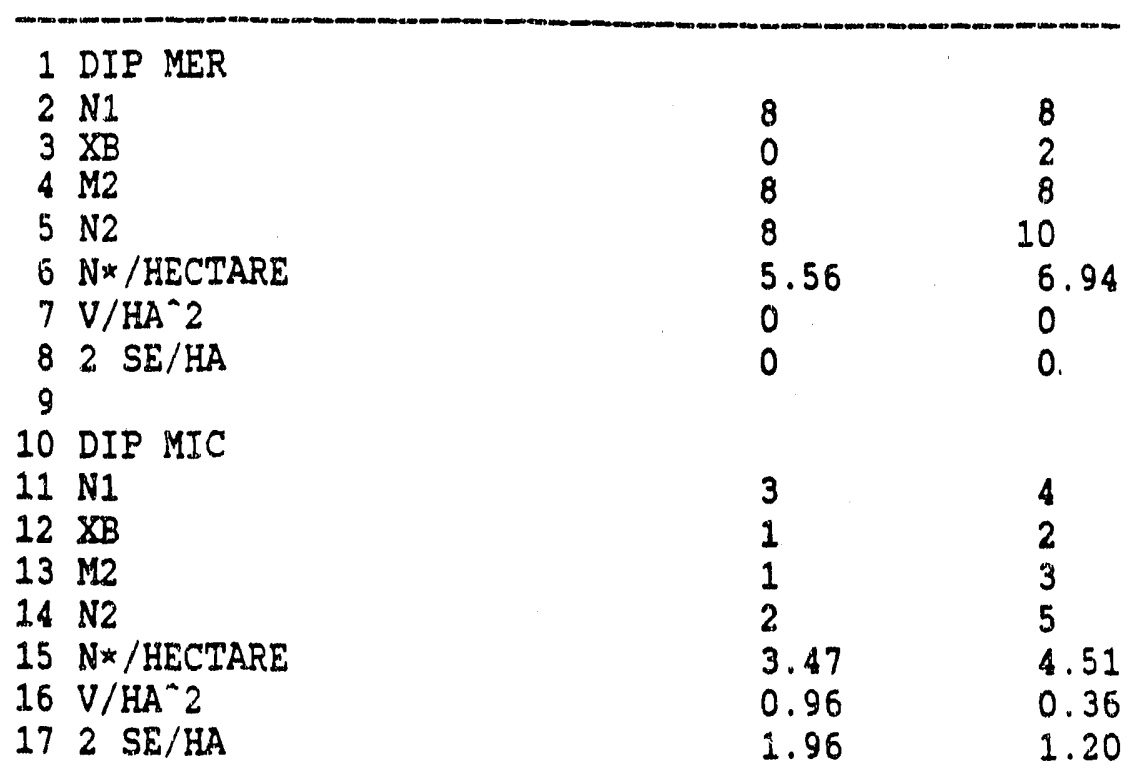


Appendix 4C (Continued)

\#MER38 8SUMDEN

0

$\begin{array}{llllll}1 & 21-J U L-88 & 2 & 22-J U L-88 & 3 & 26-J U L-88\end{array}$

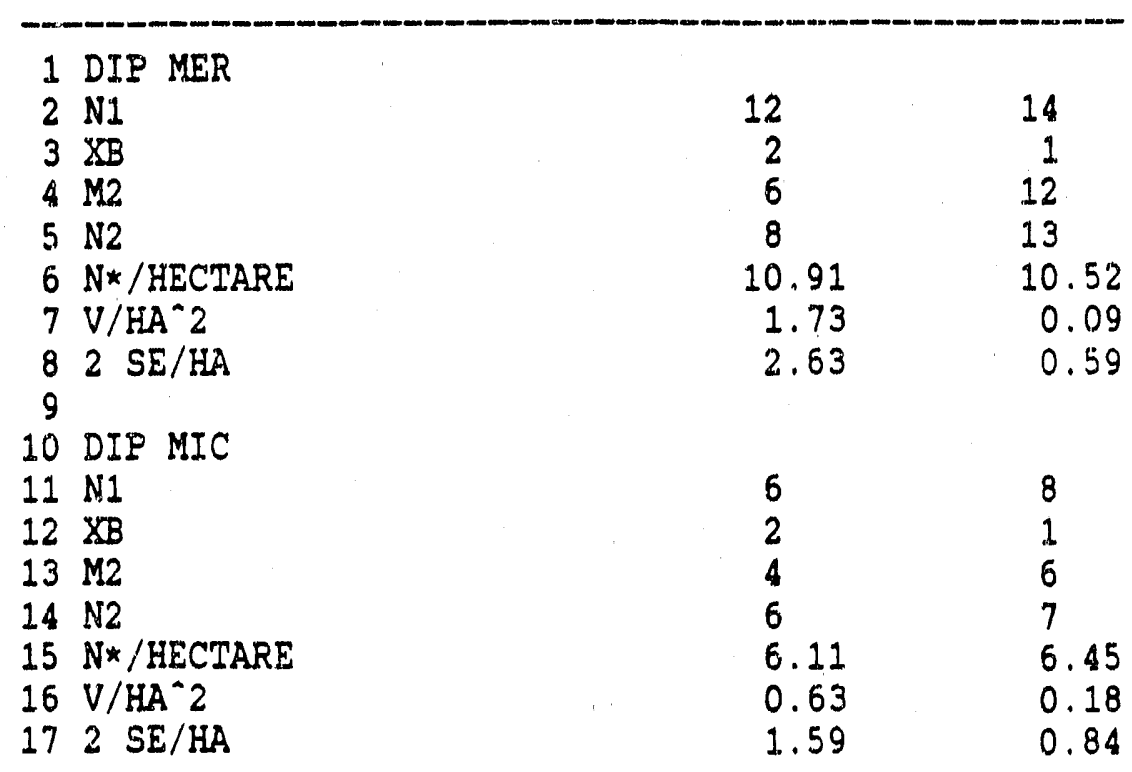

\#MID288SPRDEN

0

$\begin{array}{llllll}1 & 03-J U N-88 & 2 & 07-J U N-88 & 3 & 08-J U N-88\end{array}$

\begin{tabular}{|c|c|c|}
\hline \multicolumn{3}{|l|}{1 DIP MER } \\
\hline $2 \mathrm{~N} 1$ & 7 & 15 \\
\hline $3 \mathrm{XB}$ & 8 & 0 \\
\hline $4 M 2$ & 7 & 12 \\
\hline $5 \mathrm{~N} 2$ & 15 & 12 \\
\hline $6 \mathrm{~N} * / \mathrm{HECTARE}$ & 8.89 & 8.89 \\
\hline $7 \mathrm{~V} / \mathrm{HA} \wedge 2$ & 0 & 0 \\
\hline $82 \mathrm{SE} / \mathrm{HA}$ & 0 & 0 \\
\hline 9 & & \\
\hline 10 PER LON & & \\
\hline $11 \mathrm{N1}$ & 3 & 6 \\
\hline $12 X B$ & 3 & 0 \\
\hline $13 \mathrm{M} 2$ & 1 & 2 \\
\hline $14 \mathrm{~N} 2$ & 4 & 2 \\
\hline $15 \mathrm{~N} * /$ HECTARE & 5.33 & 3.56 \\
\hline $16 \mathrm{~V} / \mathrm{HA}^{\wedge} 2$ & 3.51 & 0 \\
\hline $172 \mathrm{SE} / \mathrm{HA}$ & 3.75 & 0 \\
\hline
\end{tabular}


Appendix 4C (Continued)

\section{\#MID388SPRDEN}

0

$1 \quad 03-J U N-88 \quad 2 \quad 07-J U N-88 \quad 3 \quad 08-J U N-88$

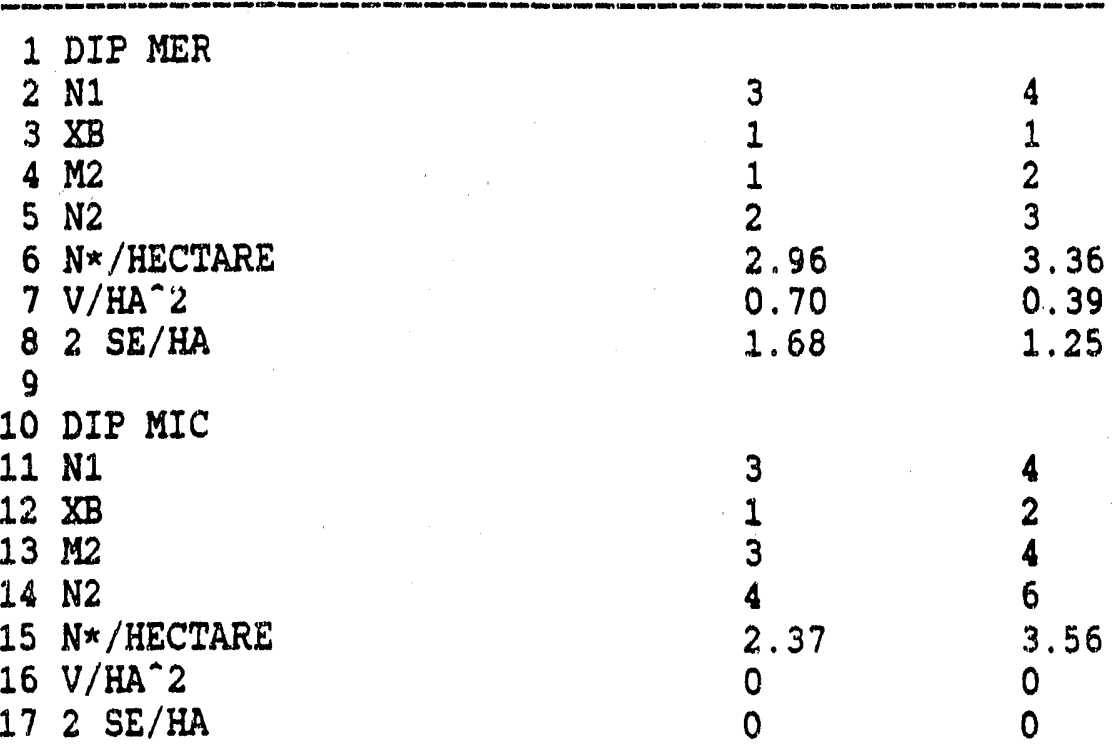

18

19 PER LON

$20 \mathrm{~N} 1$

$21 \mathrm{XB}$

$22 \mathrm{M2}$

$23 \mathrm{~N} 2$

$24 \mathrm{~N} * /$ HECTARE

$25 \mathrm{~V} / \mathrm{HA}{ }^{*} 2$

262 SE/HA

$\begin{array}{ll}2 & 2 \\ 0 & 4 \\ 1 & 2 \\ 1 & 6 \\ 1.19 & 3.56 \\ 0 & 0 \\ 0 & 0\end{array}$

27

28 PER MAN

$29 \mathrm{~N} 1$

$30 \mathrm{XB}$

$31 \mathrm{M} 2$

$32 \mathrm{~N} 2$

$33 \mathrm{~N} * /$ HEC SARE

$34 \mathrm{~V} / \mathrm{HA}^{-} 2$

$352 \mathrm{SE} / \mathrm{HA}$

3
2
1
3
4.14
1.87
2.73


Appendix 4C (Continued)

\#PAM188SPRDEN

0

$\begin{array}{llllll}2 & 24-J U N-88 & 2 & 28-J U N-88 & 3 & 29-J U N-88\end{array}$

\section{PER PAR}

2 N1

$3 \mathrm{XB}$

$4 \mathrm{M} 2$

5 N2

$6 \mathrm{N*} / \mathrm{HECTARE}$

$7 \mathrm{~V} / \mathrm{HA} 22$

$82 \mathrm{SE} / \mathrm{HA}$

9

10 PER SPP

$11 \mathrm{~N} 1$

$12 \mathrm{XB}$

$13 \mathrm{M} 2$

$14 \mathrm{~N} 2$

$15 N * /$ HECTARE

$16 \mathrm{~V} / \mathrm{HA}{ }^{\wedge} 2$

$172 \mathrm{SE} / \mathrm{HA}$

$\begin{array}{rr}10 & 25 \\ 15 & 8\end{array}$

$8 \quad 22$

$23 \quad 30$

$8.74 \quad 10.51$

0.93

1.93

0.15

0.76

$\begin{array}{rr}6 & 12 \\ 6 & 1 \\ 5 & 6 \\ 11 & 7 \\ 4.01 & 4.28 \\ 0.19 & 0.15 \\ 0.87 & 0.78\end{array}$


Appendix 4C (Continued)

\#RAM188SUMDEN

0

$\begin{array}{llllll}1 & 02-A U G-88 & 2 & 03-A U G-88 & 3 & 04-A U G-88\end{array}$

\section{PER SPP}

$2 \mathrm{~N} 1$

$3 \mathrm{XB}$

$4 \mathrm{M} 2$

$5 \mathrm{~N} 2$

$6 \mathrm{~N} * /$ HECTARE

$7 \mathrm{~V} / \mathrm{HA}^{\wedge} 2$

$82 \mathrm{SE} / \mathrm{HA}$

9

10 PER TRU

$11 \mathrm{~N} 1$

$12 \mathrm{XB}$

$13 \mathrm{M} 2$

$14 \mathrm{~N} 2$

$15 \mathrm{~N} * /$ HECTARE

$16 \mathrm{~V} / \mathrm{HA}^{-} 2$

$172 \mathrm{SE} / \mathrm{HA}$

18

19 TAM DOR

$20 \mathrm{N1}$

$2.1 \mathrm{XB}$

22 M2

$23 \mathrm{~N} 2$

$24 \mathrm{~N} * /$ HECTARE

$25 \mathrm{~V} / \mathrm{HA}^{-2} 2$

$262 \mathrm{SE} / \mathrm{HA}$

$\begin{array}{rl}11 & 15 \\ 4 & 1 \\ 9 & 8 \\ 13 & 9 \\ 4.88 & 5.18 \\ 0.12 & 0.13 \\ 0.68 & 0.73\end{array}$

$\begin{array}{ll}3 & 5 \\ 2 & 0 \\ 2 & 1 \\ 4 & 1 \\ 1.75 & 1.54 \\ 0.11 & 0 \\ 0.65 & 0\end{array}$

.

6
2
0
2
6.17
12.00
6.93


Appendix 4C (Continued)

\#RED188 JUIDEN

0

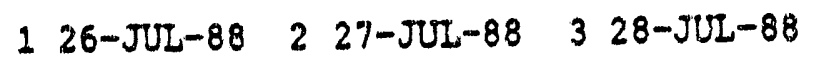

\begin{tabular}{|c|c|}
\hline $\begin{array}{l}1 \\
2 \\
3 \\
4 \\
5 \\
6 \\
7 \\
8 \\
9 \\
10 \\
11 \\
12 \\
13 \\
14 \\
15 \\
16 \\
17 \\
18 \\
19 \\
20 \\
21 \\
22 \\
23 \\
24 \\
25 \\
26\end{array}$ & 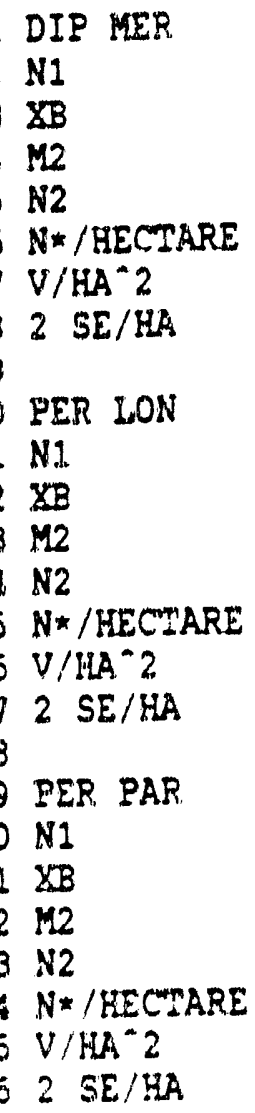 \\
\hline
\end{tabular}

20

3

16

19

15.05

0.39

1.25

2

2

0

2

5.08

7.26

5.39

2

4

1

5.08

2.42

3.11
23

1

17

15.50

0.23

0.97

4
1
1
2
4.13
1.51
2.46

6

1

6

7

4.44

0

0 
Appendix 4C (Continued)

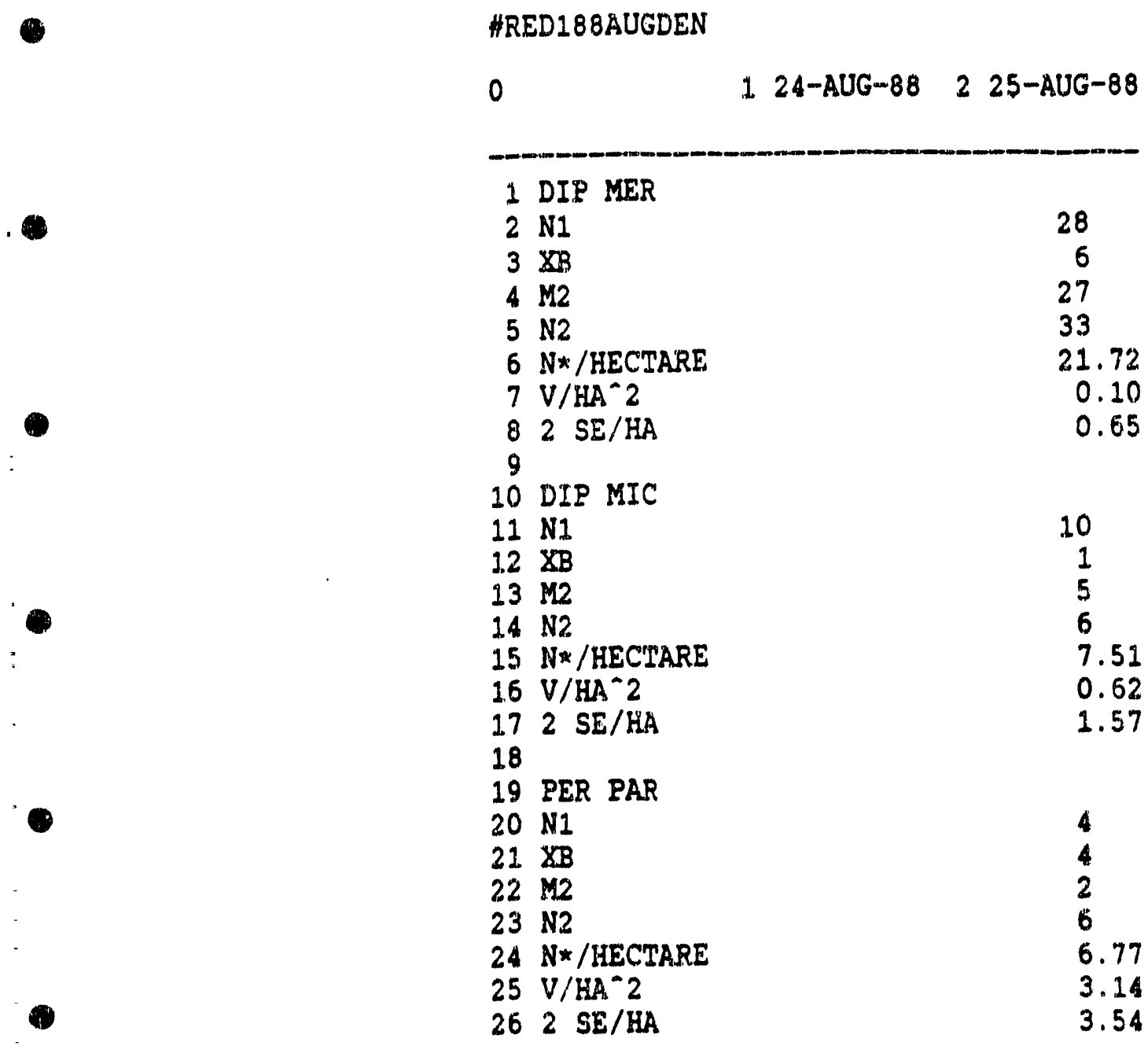


Appendix 4C (Continued)

\#RED1880CTDEN

0

$\begin{array}{llllll}125-0 C T-88 & 2 & 26-0 C T-88 & 3 & 27-O C T-88\end{array}$

\begin{tabular}{|c|c|c|}
\hline \multicolumn{3}{|l|}{1 DIP MER } \\
\hline $2 \mathrm{N1}$ & 23 & 28 \\
\hline $3 \times B$ & 5 & 6 \\
\hline $4 M 2$ & 20 & 22 \\
\hline $5 \mathrm{~N} 2$ & 25 & 28 \\
\hline $6 \mathrm{~N} * /$ HECTARE & 18.23 & 22.58 \\
\hline $7 \mathrm{~V} / \mathrm{HA}^{-2} 2$ & 0.39 & 0.96 \\
\hline $\begin{array}{l}82 \mathrm{SE} / \mathrm{HA} \\
9\end{array}$ & 1.25 & 1.96 \\
\hline 10 DIP MIC & & \\
\hline $11 \mathrm{~N} 1$ & 8 & 8 \\
\hline $12 \times 8$ & 0 & 2 \\
\hline $13 \mathrm{M} 2$ & 8 & 8 \\
\hline $14 \mathrm{N2}$ & 8 & 10 \\
\hline $15 \mathrm{N*} / \mathrm{HECTARE}$ & 5.08 & 8.35 \\
\hline $16 \mathrm{~V} / \mathrm{HA}{ }^{\wedge} 2$ & 0 & 0 \\
\hline $172 \mathrm{SE} / \mathrm{HA}$ & 0 & 0 \\
\hline
\end{tabular}


Appendix 4C (Continued)

\begin{tabular}{|c|c|c|c|}
\hline $\begin{array}{l}1 \text { DIP MER } \\
2 \mathrm{~N} 1\end{array}$ & & & \\
\hline & & 21 & 27 \\
\hline $\begin{array}{l}3 \mathrm{XB} \\
4 \mathrm{M} 2\end{array}$ & & $\begin{array}{r}6 \\
17\end{array}$ & $\begin{array}{r}4 \\
10\end{array}$ \\
\hline $\begin{array}{l}4 \mathrm{M} 2 \\
5 \mathrm{~N} 2\end{array}$ & & $\begin{array}{l}17 \\
23\end{array}$ & $\begin{array}{l}19 \\
23\end{array}$ \\
\hline $6 \mathrm{~N}^{*} / \mathrm{HECTARE}$ & & 17.99 & $\begin{array}{l}23 \\
20.70\end{array}$ \\
\hline $7 \mathrm{~V} / \mathrm{HA}-2$ & & 0.83 & 1.03 \\
\hline $82 \mathrm{SE} / \mathrm{HA}$ & & 1.82 & \\
\hline${ }_{10}^{9}$ DIP MIC & & & \\
\hline $\begin{array}{l}10 \text { DIP MIC } \\
11 \mathrm{~N} 1\end{array}$ & & & \\
\hline $12 \mathrm{XB}$ & & 3 & $\begin{array}{l}6 \\
2\end{array}$ \\
\hline $13 \mathrm{M2}$ & & 1 & 1 \\
\hline & & 4 & 3 \\
\hline $15 \mathrm{~N} /$ HECTARE & & 5.71 & 8.25 \\
\hline $16 \mathrm{~V} / \mathrm{HA} \wedge 2$ & & 4.03 & 9.41 \\
\hline $172 \mathrm{SE} / \mathrm{HA}$ & & 4.02 & \\
\hline 18 & & & \\
\hline 19 PER LON & & & \\
\hline $20 \mathrm{~N} 1$ & & 3 & $\begin{array}{l}6 \\
0\end{array}$ \\
\hline $21 X B$ & & 3 & 0 \\
\hline 22.2 & & 1 & 1 \\
\hline $23 \mathrm{~N} 2$ & & 4 & \\
\hline $24 \mathrm{~N} /$ HECTARE & & 5.71 & 3.81 \\
\hline $25 \mathrm{~V} / \mathrm{HA}^{-}-2$ & & 4.03 & 0 \\
\hline $262 \mathrm{SE} / \mathrm{HA}$ & & 4.02 & 0 \\
\hline 27 & & & \\
\hline 28 PER PAR & & 2 & \\
\hline $\begin{array}{l}29 \mathrm{N1} \\
30 \mathrm{XB}\end{array}$ & & 4 & 1 \\
\hline $\begin{array}{l}30 \mathrm{XB} \\
31 \mathrm{M} 2\end{array}$ & & $i$ & 2 \\
\hline $\begin{array}{ll}31 & M 2 \\
32 & N 2\end{array}$ & & 5 & 3 \\
\hline $33 \mathrm{~N} * /$ HECTARE & & 5.08 & 5.29 \\
\hline $34 \mathrm{~V} / \mathrm{HA}^{\wedge} 2$ & & 2.42 & 1.25 \\
\hline $352 \mathrm{SE} / \mathrm{HA}$ & & 3.11 & 2.24 \\
\hline
\end{tabular}


Appendix 4C (Continued)

\#RED288AUGDEN

0

$\begin{array}{llll}24-A U G-88 & 2 & 25-A U G-88\end{array}$

$\begin{array}{llr}1 & \text { DIP MER } & \\ 2 & \text { N1 } & 22 \\ 3 \text { XB } & 7 \\ 4 \text { M2 } & 17 \\ 5 & \text { N2 } & 24 \\ 6 & N * / H E C T A R E & 19.65 \\ 7 \text { V/HA } 2 & 1.32 \\ 8 & 2 \text { SE/HA } & 2.30 \\ 9 & \\ 10 & \text { DIP MIC } & \\ 11 \text { N1 } & 7 \\ 12 \text { XB } & 2 \\ 13 \text { M2 } & 6 \\ 14 \text { N2 } & 8 \\ 15 \text { N*/HECTARE } & 5.90 \\ 16 \text { V/HA 2 } & 0.15 \\ 172 \text { SE/HA } & 0.77\end{array}$

\#RED2880CTDEN

0

$125-O C T-88 \quad 2 \quad 26-O C T-88 \quad 3 \quad 27-0 C T-88$

\begin{tabular}{|c|c|c|}
\hline \multicolumn{3}{|l|}{1 DIP MER } \\
\hline $2 \mathrm{N1}$ & 24 & 30 \\
\hline $3 X B$ & 6 & 2 \\
\hline $4 \mathrm{M} 2$ & 19 & 22 \\
\hline $5 \mathrm{~N} 2$ & 25 & 24 \\
\hline $6 \mathrm{~N} * /$ HECTARE & 20.00 & 20.76 \\
\hline $7 \mathrm{~V} / \mathrm{HA}-2$ & 0.94 & 0.39 \\
\hline $82 \mathrm{SE} / \mathrm{HA}$ & 1.93 & 1.25 \\
\hline 10 DIP MIC & & \\
\hline $\begin{array}{l}10 \mathrm{DIP} M I C \\
11 \mathrm{NI}\end{array}$ & & \\
\hline $1.1 \mathrm{~N} 1$ & 13 & 16 \\
\hline $12 \mathrm{XB}$ & 3 & 3 \\
\hline $13 M 2$ & 12 & 15 \\
\hline $14 \mathrm{~N} 2$ & 15 & 18 \\
\hline $15 \mathrm{~N} * /$ HECTARE & 10.31 & 12.18 \\
\hline $16 \mathrm{~V} / \mathrm{HA}^{-} 2$ & 0.11 & 0.09 \\
\hline $172 \mathrm{SE} / \mathrm{HA}$ & 0.58 & 0.60 \\
\hline
\end{tabular}


Appendix 4C (Continued)

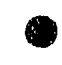

\#ROV788SPRDEN

0

$1 \quad 14-J U N-88 \quad 2 \quad 15-J U N-88 \quad 3 \quad 16-J U N-88$

\begin{tabular}{|c|c|c|}
\hline \multicolumn{3}{|l|}{1 DIP MER } \\
\hline $2 \mathrm{~N}=$ & 9 & 11 \\
\hline $3 \mathrm{XB}$ & 2 & 3 \\
\hline $4 \mathrm{M2}$ & 7 & 10 \\
\hline & 9 & 13 \\
\hline $6 \mathrm{~N} /$ HECTARE & 3.55 & 4.41 \\
\hline $7 \mathrm{~V} / \mathrm{HA}^{*} 2$ & 0.07 & $\begin{array}{l}0.03 \\
0.36\end{array}$ \\
\hline $\begin{array}{l}82 \mathrm{SE} / \mathrm{HA} \\
9\end{array}$ & 0.51 & 0.36 \\
\hline \multicolumn{3}{|l|}{${ }_{10}^{9}$ DIP MIC } \\
\hline $\begin{array}{l}11 \mathrm{N1} \\
12 \mathrm{XB}\end{array}$ & 23 & 33 \\
\hline $\begin{array}{l}12 \mathrm{XB} \\
13 \mathrm{M}\end{array}$ & 10 & 5 \\
\hline $13 \mathrm{M}$ & 19 & 31 \\
\hline $\begin{array}{l}14 \mathrm{~N} 2 \\
15 \mathrm{~N} / \mathrm{HECTARE}\end{array}$ & 29 & 36 \\
\hline $15 \mathrm{~N} * /$ HECTARE & 10.80 & 11.82 \\
\hline $\begin{array}{l}16 \mathrm{~V} / \mathrm{HA}^{*} 2 \\
172 \mathrm{SE} / \mathrm{HA}\end{array}$ & 0.33 & 0.04 \\
\hline $\begin{array}{l}172 \mathrm{SE} / \mathrm{H} / \\
18\end{array}$ & 1.14 & 0.38 \\
\hline \multicolumn{3}{|l|}{$\begin{array}{l}18 \\
19 \text { PER LON }\end{array}$} \\
\hline $20 \mathrm{N1}$ & 15 & 31 \\
\hline $21 X B$ & 16 & 2 \\
\hline $22 \mathrm{M} 2$ & 11 & 22 \\
\hline $23 \mathrm{~N} 2$ & 27 & 24 \\
\hline $24 \mathrm{~N} * / \mathrm{HECTARE}$ & 11.21 & 10.43 \\
\hline $25 \mathrm{~V} / \mathrm{HA}^{\wedge} 2$ & 1.46 & 0.11 \\
\hline $262 \mathrm{SE} / \mathrm{HA}$ & 2.42 & 0.66 \\
\hline \multicolumn{3}{|l|}{$\begin{array}{l}27 \\
28 \text { ONY TOR }\end{array}$} \\
\hline 28 ONY TOR & & \\
\hline $29 \mathrm{~N} 1$ & $\begin{array}{l}2 \\
8\end{array}$ & $\begin{array}{r}10 \\
0\end{array}$ \\
\hline $30 \mathrm{YB}$ & $\begin{array}{l}8 \\
1\end{array}$ & 2 \\
\hline $31 \mathrm{M} 2$ & $\begin{array}{l}1 \\
9\end{array}$ & 2 \\
\hline $32 \mathrm{~N} 2$ & $\begin{array}{l}9 \\
4.32\end{array}$ & 3.09 \\
\hline $\begin{array}{l}33 \mathrm{~N} * / \mathrm{HECTARE} \\
34 \mathrm{~V} / \mathrm{HA} A^{\wedge} 2\end{array}$ & 1.91 & 0 \\
\hline $352 \mathrm{SE} / \mathrm{HA}$ & 2.76 & 0 \\
\hline
\end{tabular}


Appendix 4C (Continued)

\#ROV888SPRDEN

0

1. $14-J U N-88 \quad 2 \quad 15-J U N-88 \quad 3 \quad 16-J U N-88$

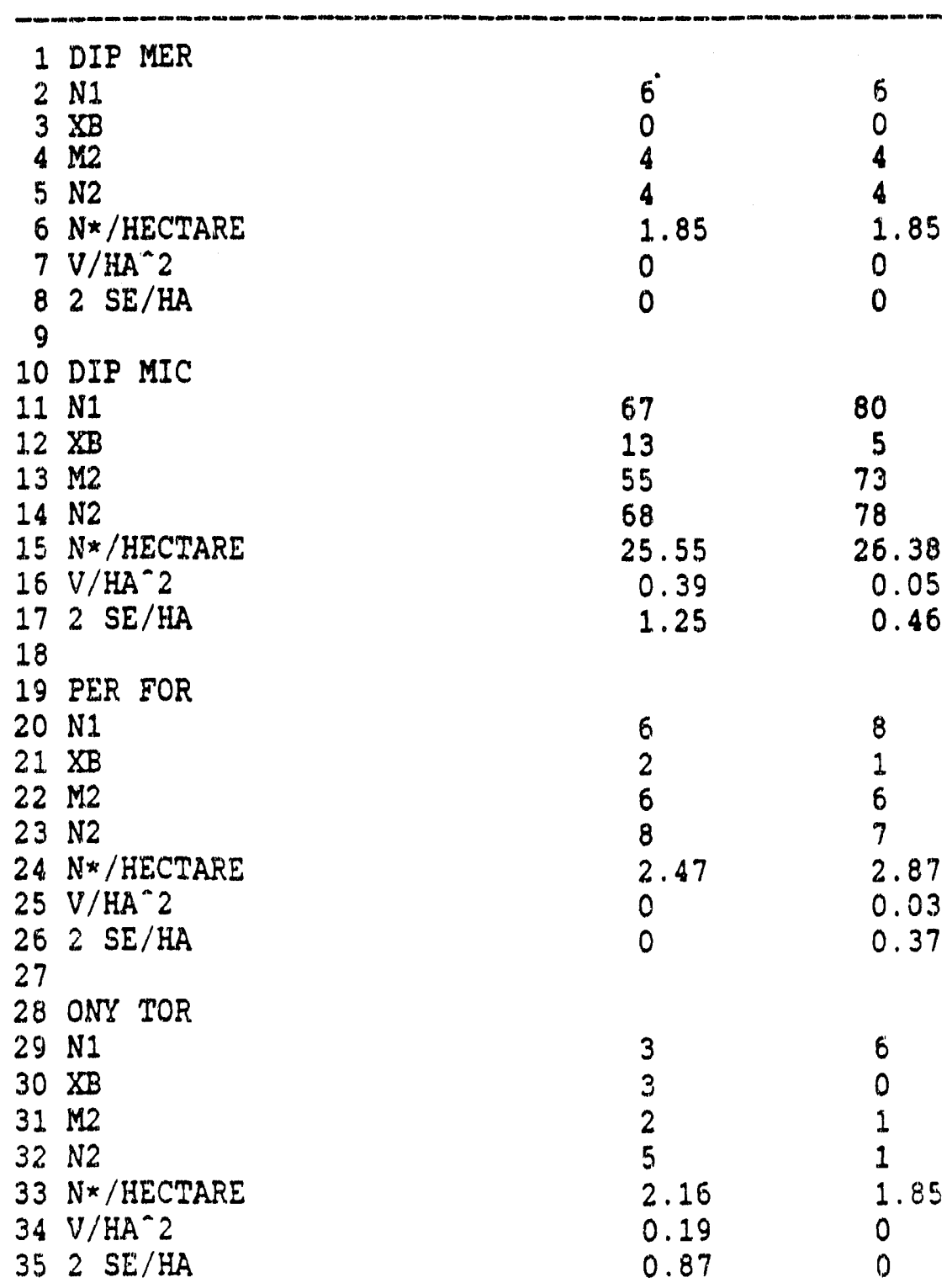


Appendix 4C (Continued)

-

\#YUF 188SPRDEN

0

$\begin{array}{llllll}26-A P R & 288 & 2 & 27-A P R-88 & 3 & 28-A P R-88\end{array}$

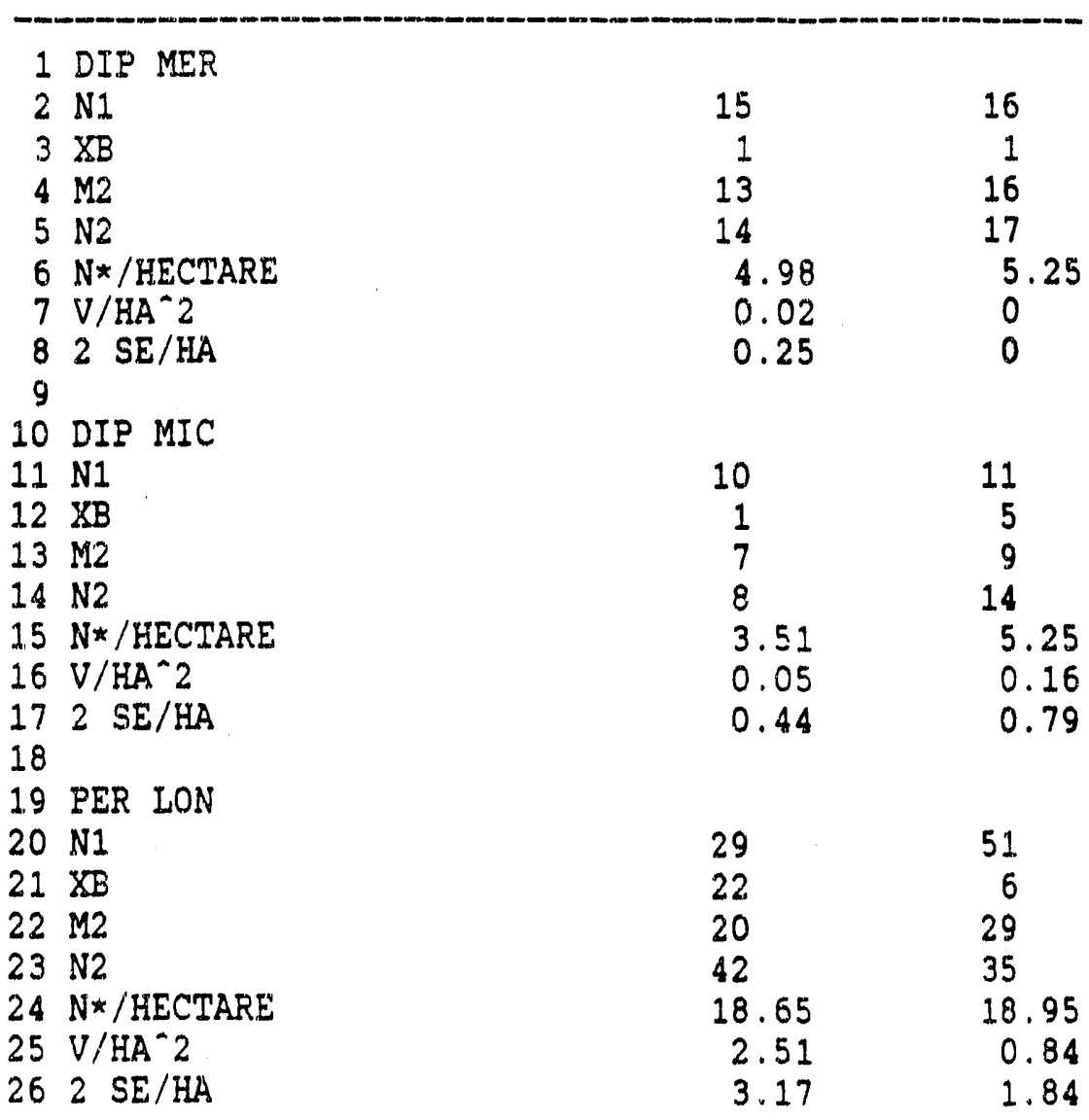


Appendix 4C (Continued)

\#YUF988SPRDEN

0

$104-M A Y-88 \quad 2 \quad 16-M A Y-88 \quad 3 \quad 17-M A Y-88 \quad 4 \quad 18-M A Y-88$

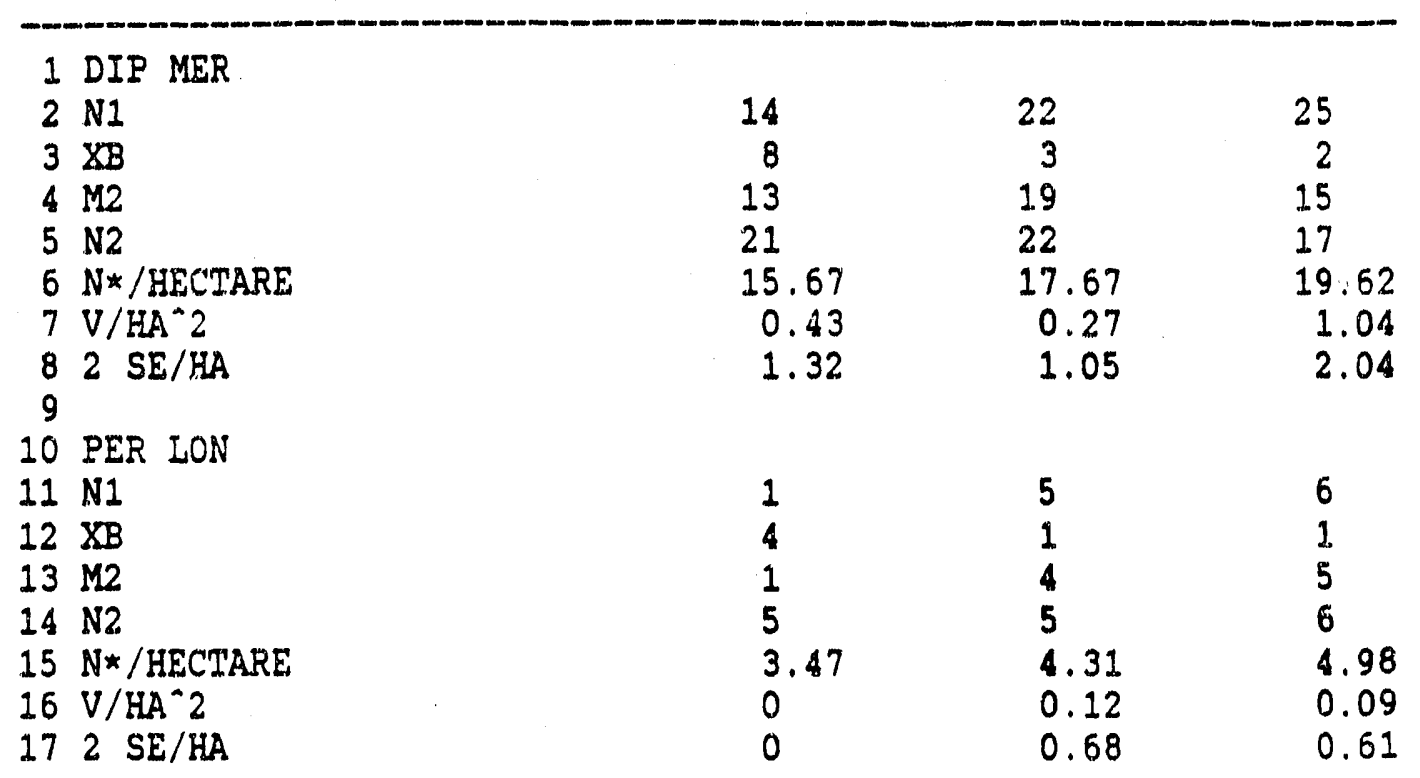

\#YUF988DIPMERSUNDEN

$0 \quad 1$ O9-AUG-88 2 10-AUG-88

\begin{tabular}{|c|c|}
\hline $\begin{array}{ll}1 & N 1 \\
2 & X B \\
3 & M 2 \\
4 & N 2 \\
5 & N * / H E C T A R E \\
6 & V / H A * 2 \\
7 & 2 \quad S E / H A\end{array}$ & $\begin{array}{l}26 \\
8 \\
20 \\
28 \\
25.20 \\
1.87 \\
2.73\end{array}$ \\
\hline
\end{tabular}


Appendix 4C (Continued)

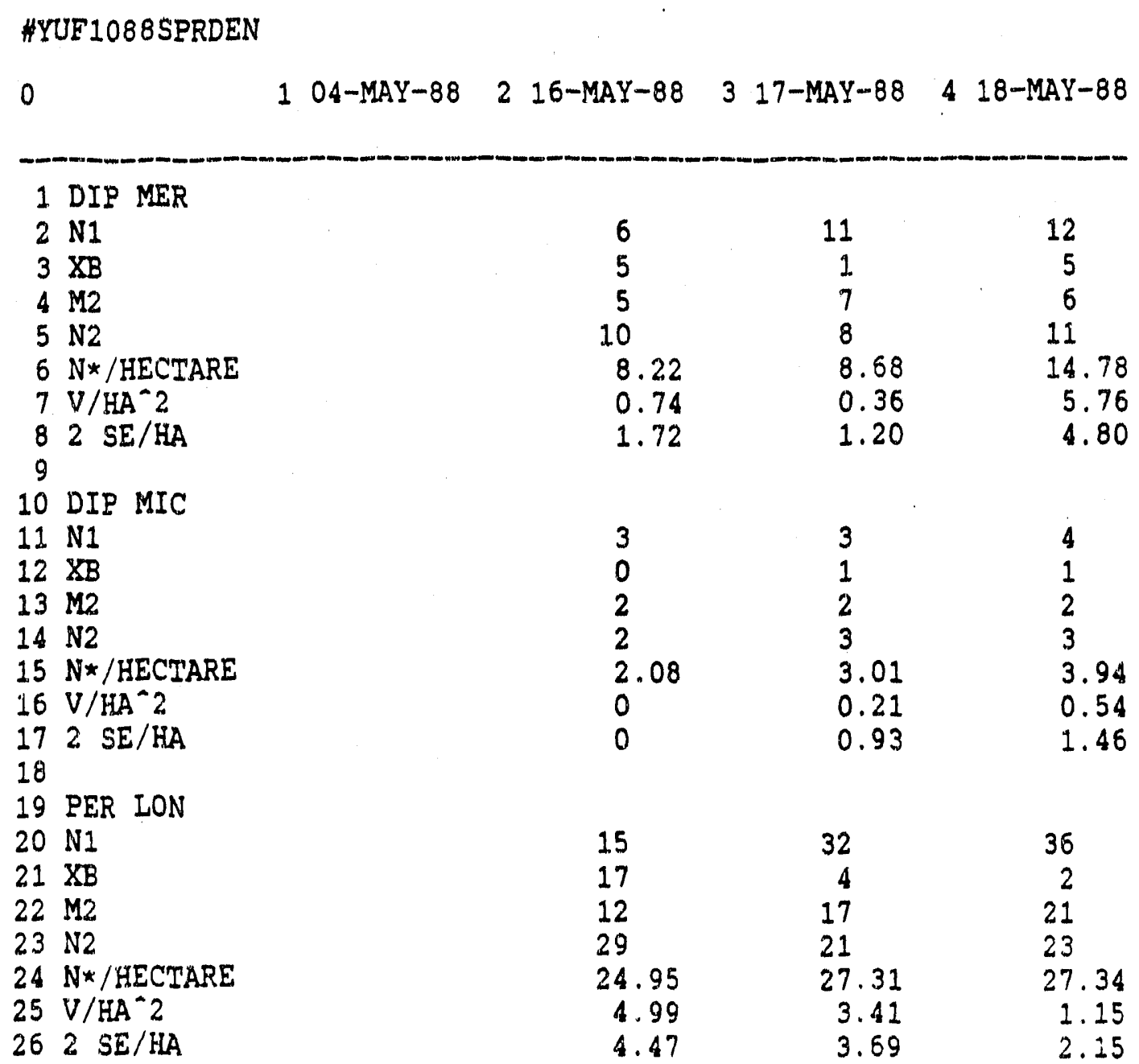

\#YUF1088DIPMERSUMDEN

$\begin{array}{lllll}0 & 1 & 09-A U G-88 & 2 & 10-A U G-88\end{array}$

$\begin{array}{llr}1 & \mathrm{~N} 1 & 8 \\ 2 & \mathrm{XB} & 4 \\ 3 \mathrm{M} 2 & 7 \\ 4 \mathrm{~N} 2 & 11 \\ 5 \mathrm{~N} \times / \mathrm{HECTARE}^{\wedge} & 8.68 \\ 6 \mathrm{~V} / \mathrm{HA}^{\wedge} 2 & 0.36 \\ 72 \mathrm{SE} / \mathrm{HA} & 1.20\end{array}$


Appendix 4C (Continued)

\#YUE1188DIPMERSPRDEN

0

$120-$ MAY-88 225 25-MAY-88 3 26-MAY-88

\begin{tabular}{llll}
\hline $\mathrm{N} 1$ & 6 & 7 \\
$2 \mathrm{XB}$ & 1 & 1 \\
$3 \mathrm{M} 2$ & 6 & 6 \\
$4 \mathrm{~N} 2$ & 7 & 7 \\
$5 \mathrm{~N} \times / \mathrm{HECTARE}$ & 4.44 & 5.17 \\
$6 \mathrm{~V} / \mathrm{HA} 2$ & 0 & 0.07 \\
$72 \mathrm{SE} / \mathrm{HA}$ & 0 & 0.51
\end{tabular}

\#YUF1288SPRDEN

0

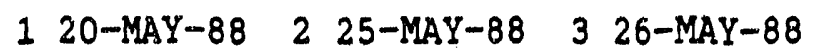

\begin{tabular}{|c|c|c|}
\hline \multicolumn{3}{|l|}{1 DIP MER } \\
\hline \multicolumn{3}{|l|}{$2 \mathrm{~N} 1$} \\
\hline $3 \mathrm{XB}$ & 4 & 0 \\
\hline $4 \mathrm{M} 2$ & 11 & 7 \\
\hline $5 \mathrm{~N} 2$ & & 7 \\
\hline $6 \mathrm{~N} * / \mathrm{HECTARE}$ & 11.22 & 10.79 \\
\hline $7 \mathrm{~V} / \mathrm{HA}{ }^{\wedge} 2$ & 0.39 & 0 \\
\hline $82 \mathrm{SE} / \mathrm{HA}$ & 1.24 & 0 \\
\hline 9 & & \\
\hline 10 DIP MIC & & \\
\hline $11 \mathrm{N1}$ & 2 & 5 \\
\hline $12 \mathrm{XB}$ & 3 & 1 \\
\hline $13 \mathrm{M} 2$ & 1 & 3 \\
\hline $14 \mathrm{~N} 2$ & & 4 \\
\hline $15 N * /$ HECTARE & 4.13 & 4.13 \\
\hline $16 \mathrm{~V} / \mathrm{HA}^{-2} 2$ & 1.51 & 0.30 \\
\hline $172 \mathrm{SE} / \mathrm{HA}$ & 2.46 & 1.10 \\
\hline 18 & & \\
\hline 19 PER LON & & \\
\hline $20 \mathrm{~N} 1$ & 11 & 18 \\
\hline $21 \mathrm{XB}$ & 7 & 1 \\
\hline $22 \mathrm{M} 2$ & 8 & 9 \\
\hline $23 \mathrm{~N} 2$ & 15 & 10 \\
\hline $24 N * /$ HECTARE & 12.91 & 12.63 \\
\hline $25 \mathrm{~V} / \mathrm{HA}^{-} 2$ & 2.01 & 0.69 \\
\hline 262 SE/HA & 2.83 & 1.66 \\
\hline
\end{tabular}


Appendix 4C (Continued)

\begin{tabular}{|c|c|c|c|}
\hline \multicolumn{4}{|c|}{ \#YUF1388DIPMERSPRDEN } \\
\hline 0 & $120-M A Y-88$ & $225-M A Y-88$ & $326-M A Y-88$ \\
\hline 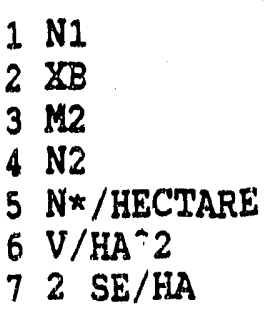 & & $\begin{array}{l}11 \\
1 \\
11 \\
12 . \\
7.62 \\
0 \\
0\end{array}$ & $\begin{array}{c}12 \\
1 \\
8 \\
9 \\
8.54 \\
0.26 \\
1.02\end{array}$ \\
\hline
\end{tabular}

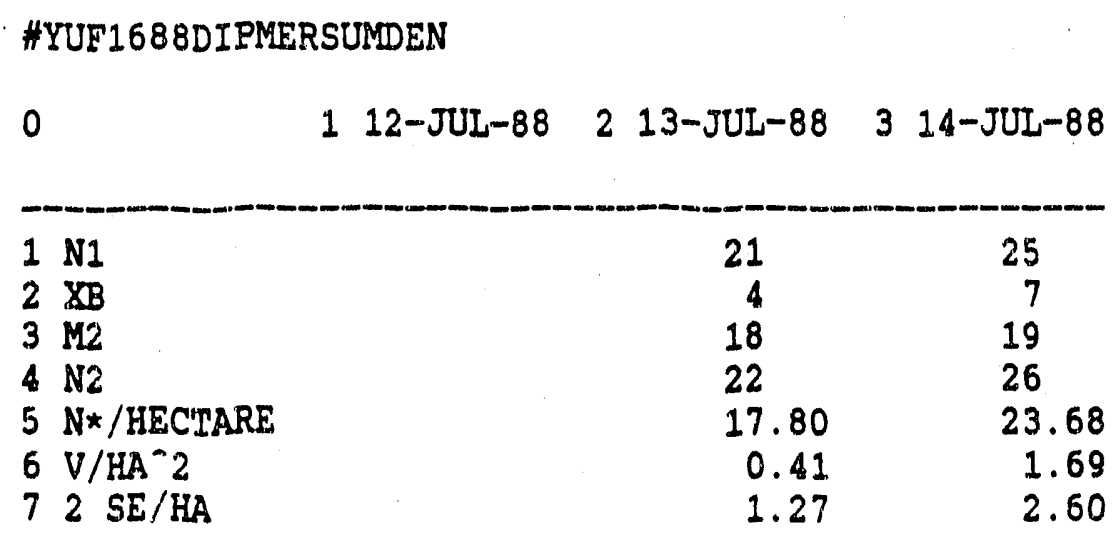

\#YUE'1788DIPMERSUMDEN

$\begin{array}{lllllll}0 & 1 & 12-J U L-88 & 2 & 13-J U L-88 & 3 & 14-J U L-88\end{array}$

\begin{tabular}{llcc}
\hline $\mathrm{N} 1$ & 23 & 29 \\
$2 \mathrm{XB}$ & 6 & 0 \\
$3 \mathrm{M} 2$ & 22 & 24 \\
$4 \mathrm{N2}$ & 28 & 24 \\
$5 \mathrm{N*} / \mathrm{HECTARE}$ & 20.32 & 20.14 \\
$6 \mathrm{~V} / \mathrm{HA} * 2$ & 0.16 & 0 \\
$72 \mathrm{SE} / \mathrm{HA}$ & 0.80 & 0
\end{tabular}


Appendix 4C (Continued)

\section{\#YUF'1888SUMDEN}

0

1 12-JUL-88 2 13-JUL-88 3 14-JUL-88

\begin{tabular}{|c|c|c|}
\hline 1 DIP MER & & \\
\hline 2 N.1 & 10 & 14 \\
\hline $3 \mathrm{XB}$ & 4 & 1 \\
\hline $4 \mathrm{M} 2$ & 7 & 9 \\
\hline 5. N2 & 11 & 10 \\
\hline $6 \mathrm{~N} * / \mathrm{HECTARE}$ & 10.76 & 10.76 \\
\hline $7 \mathrm{~V} / \mathrm{HAA}^{\wedge} 2$ & 1.33 & 0.36 \\
\hline $82 \mathrm{SE} / \mathrm{HA}$ & 2.30 & 1.20 \\
\hline 10 DIP MIC & & \\
\hline $11 \mathrm{~N} 1$ & 4 & 6 \\
\hline $12 X \mathrm{XB}$ & 2 & 2 \\
\hline $13 \mathrm{M} 2$ & 1 & 5 \\
\hline $14 \mathrm{~N} 2$ & 3 & 7 \\
\hline $15 \mathrm{~N} * / \mathrm{HECTARE}$ & 6.25 & 5.79 \\
\hline $16 \mathrm{~V} / \mathrm{HA} \leadsto 2$ & 4.82 & 0.21 \\
\hline $172 \mathrm{SE} / \mathrm{HA}$ & 4.39 & 0.93 \\
\hline 18 & & \\
\hline 19 PER ION & & \\
\hline $20 \mathrm{~N} 1$ & 3 & 5 \\
\hline $21 \times B$ & 2 & 1 \\
\hline $22 \mathrm{M} 2$ & 2 & 4 \\
\hline $23 \mathrm{~N} 2$ & 4 & 5 \\
\hline $24 \mathrm{~N} * /$ HECTARE & 3.94 & 4.31 \\
\hline $25 \mathrm{~V} / \mathrm{HA}^{\wedge} 2$ & 0.54 & 0.12 \\
\hline $262 \mathrm{SE} / \mathrm{HA}$ & 1.46 & 0.68 \\
\hline 27 & & \\
\hline 28 AMM LEU & & \\
\hline $29 \mathrm{~N} 1$ & 4 & 6 \\
\hline $30 \times B$ & 2 & 0 \\
\hline $31 \mathrm{M} 2$ & 0 & 3 \\
\hline 32 N2 & 2 & 3 \\
\hline $33 \mathrm{~N} / \mathrm{HECTARE}$ & 9.72 & 4.17 \\
\hline $34 \mathrm{~V} / \mathrm{HA} \sim 2$ & 28.94 & 0 \\
\hline $352 \mathrm{SE} / \mathrm{HA}$ & 10.76 & 0 \\
\hline
\end{tabular}




\title{
SECTION 5 WILDLIFE UTILIZATION OF NATURAL SPRINGS AND MAN-MADE WATER SOURCES AT THE NEVADA TEST SITE
}

\author{
by \\ Evan M. Romney and Paul D. Greger
}

\section{INTRODUCTION}

Wildlife utilization of water sources on the Nevada Test Site (NTS) had not been studied in any detail. Wildlife usage of permanent or temporary water sources was only mentioned briefly in existing studies (Hayward et al. 1963, Allred et al. 1963, Jorgensen and Hayward 1965, Giles 1976, Giles and Cooper 1985). Studies at natural springs were limited to Giles (1976), who examined the availability of water for wildlife usage; and Taylor and Giles (1979), who investigated algae living in those waters. The Giles study (1976) was the most relevant to our study, but it did not contain any detailed information on wildlife utilization over time. The long-term objectives of this study were to describe the kinds of wildlife that utilize the available water sources on the NTS, determine the extent of such utilization, and assess any changes and unusual disturbances in the natural spring habitats.

\section{METHODS}

Monitoring of both permanent and temporary water sources involved site visits on a seasonal basis to determine the qualitative usage of natural springs and man-made ponds by wildlife. Utilization by wildlife was identified by numbers and species, whenever possible.

Conservative estimates were provided for large numbers of mobile specimens, such as birds. Wildlife of concern included feral horses, deer, antelope, elk, mountain lion, bobcat, ringtail, coyote, badger, kit fox, and birds such as raptors and migrating waterfowl. Identification of wildlife followed the recommendations given in Burt and Grossenheider (1976) for mammals, and Peterson (1961) and Robbins et al. (1983) for birds. Following the recommendations given in Murie (1974), observed animal signs (i.e., tracks and scats) determined qualitative usage. Close examination of the surrounding riparian habitat generally provided qualitative evidence of animal usage; however, in certain cases usage of the water source also could be assumed from animal sightings made within a reasonable distance from the water source.

Detailed records were kept on the observations of feral horses, including the water sources they used and the areas they inhabited for grazing. Location, time of observation, group size, number of adults and juveniles, and sex were recorded. Individual horses were photographed when opportunity permitted for identification of specimens from which to maintain longevity records. 
Samples of riparian and aquatic vegetation growing at natural springs were collected, identified, and deposited with the BECAMP herbarium collection. Water quality (twenty-five elements) of the natural springs and man-made ponds was monitored for mineral content, determined by ICP-spectrometry (Alexander and McAnulty 1981).

Long term photographic documentation around natural springs was begun in 1988 to assess the impact of feral horses on the riparian habitat and adjacent areas from grazing and trampling. Field notes from observations were combined with the photographic record to document any change in habitat. Photographic benchmarks were used to ensure reproducibility of the subject landscape. These benchmarks were 5-foot posts of two types: one post marked the position from which each photograph was taken; the other post near the spring suprorted a white PVC pipe sleeve that is focused to appear at the same position in the frame of each successive photograph. Several photographic shots were necessary at certain places to adequately document the riparian habitat. Figure 5.1 is an example of the field note sheet used for each water source visit.

\section{RESULTS}

Figure 5.2 shows the relative distribution of natural springs monitored on the NTS that provide a supply of water for wildlife. Table 5.1 lists the elevations and Nevada grid coordinate locations for those springs. A few seepage sites also supply water during the winter and spring months but dry up during surmer and fall. Such sites were not routinely monitored.

Figure 5.3 shows the distribution of man-made water sources that presently are available to wildlife; Table 5.2 lists their elevations and Nevada grid coordinate locations. Some well water sources used for intermittent engineering activity have been and will be short-lived, but some of them have existed for many years and have developed dependent wildlife populations. All of the well ponds and reservoirs host intermittent populations of migrating waterfowl.

Table 5.3 indicates the wildlife utilization of natural springs on the NTS during the period of November 1987 through December 1988 as determined by sighting; and the pesence of animal signs such as srats and tracks. Table 5.4 lists the wildlife uilization at man-made water sources ciuring the same period. It should be noted, however, that those totals may include counting the same individual on repeated visits as certainly was the case for feral horses observed at the Camp 17 water source.

Figure 5.4 shows the relative locations of raptor sightings $(n=87)$ during the 1988 monitoring yuar. Forty-eight percent of those sightings involved individuals perched upon utility poles: most of the others were observed in fight. Red-tailad hawks were the 
dominant species observed during 1988, however other species such as golden eagles, prairie falcons and northern harriers were also observed.

Data for the mineral element concentrations determined in water samples collected from natural springs are listed in Appendix 5.

Figure 5.5 illustrates the utilization of both natural springs and man-made water sources by mule deer as determined by the presence of tracks and scats. Frequency of use was rare to low at water sources located in lower elevations of the NTS and moderate to heavy at water sources located in higher elevations.

The feral horse population made heavy utilization of several natural springs and man-made water sources located within their preferred grazing area (Figure 5.6). Individual and group sightings of feral horses were made primarily within the higher elevation areas of the NTS as illustrated in Figure 5.7. However, a few horses also were seen grazing on the grassland in Area 2. 


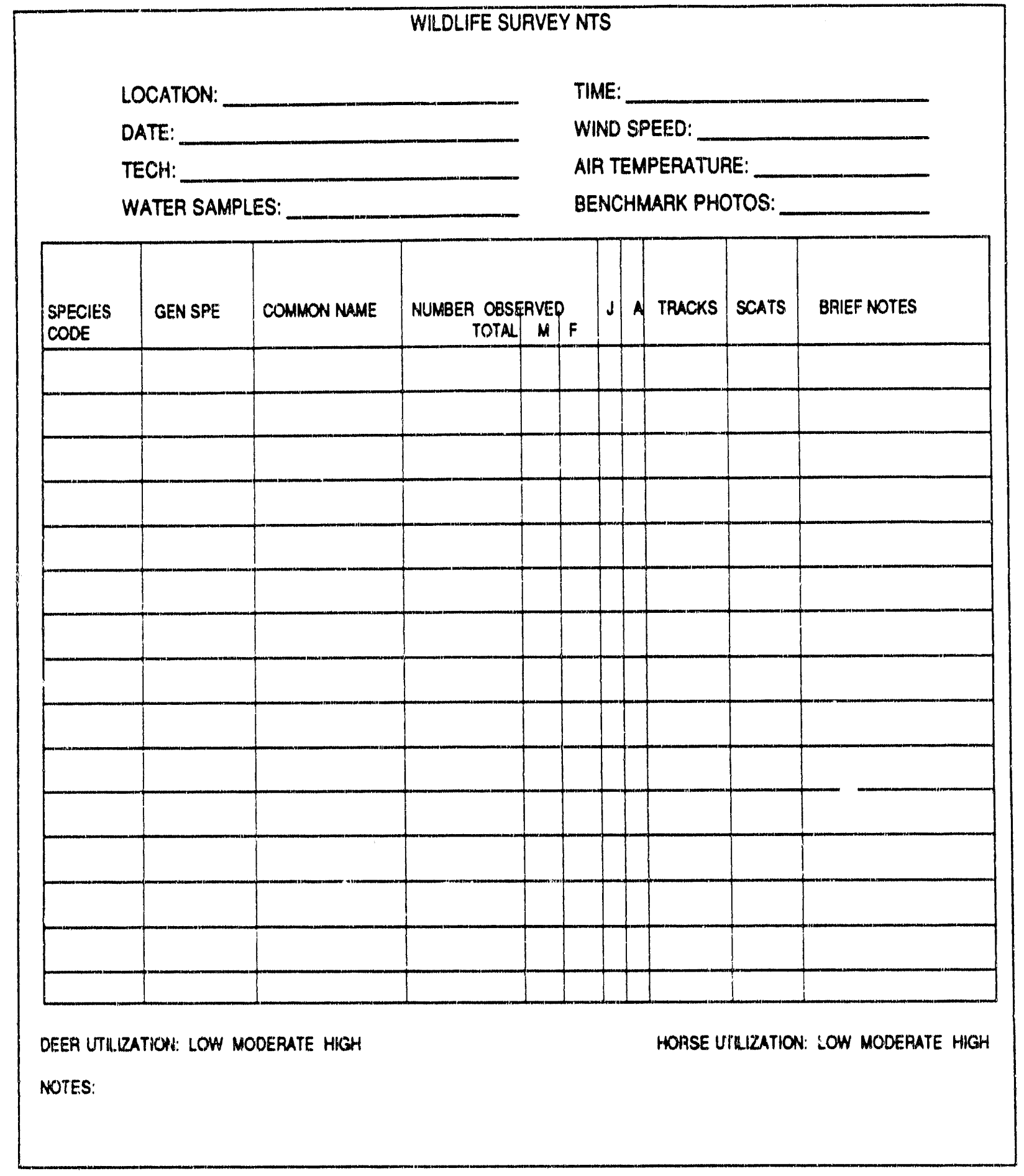

Figure 5.1. Field Data Sheet 


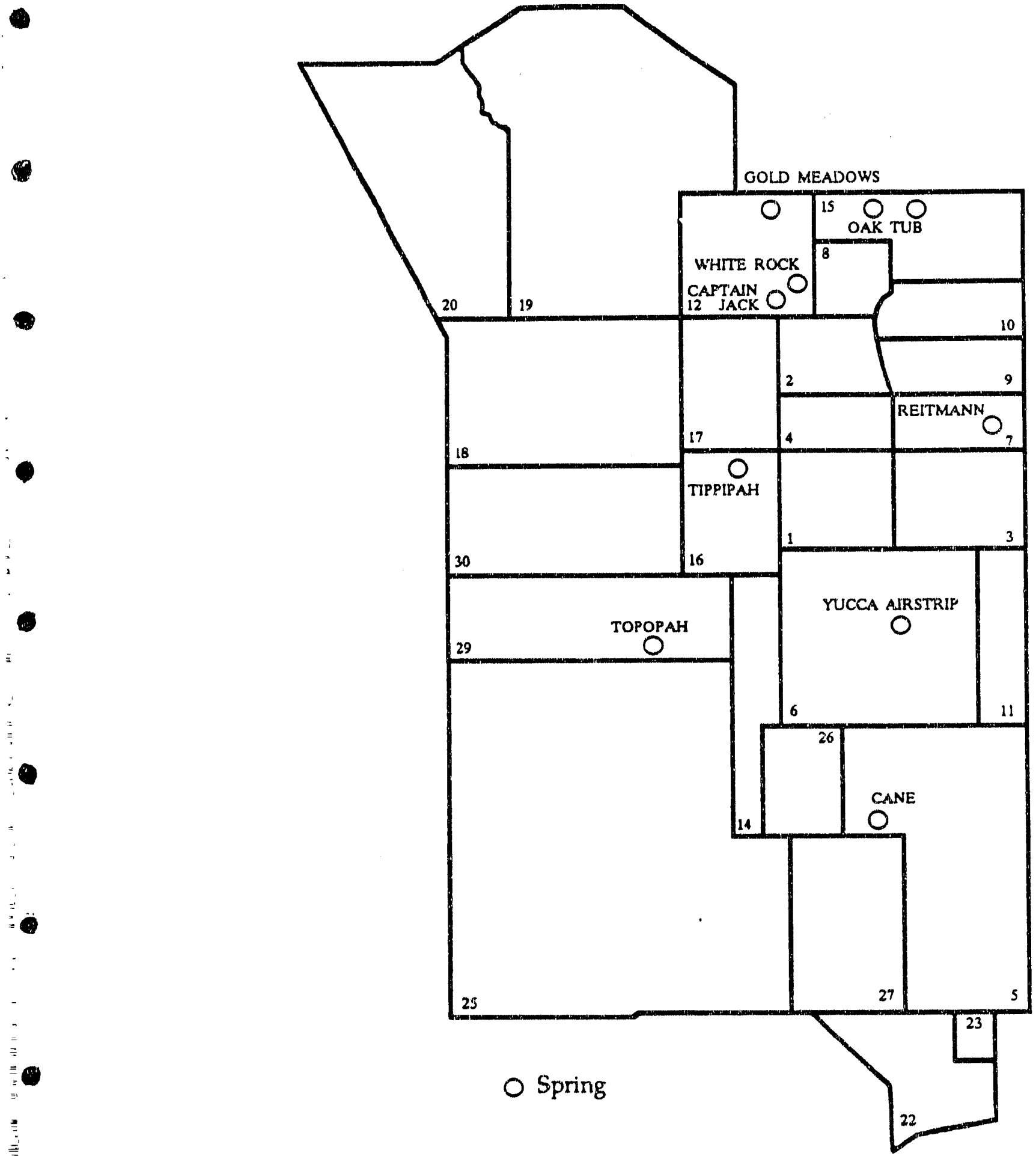

Figure 5.2. Natural springs at the Nevada Test Site. 


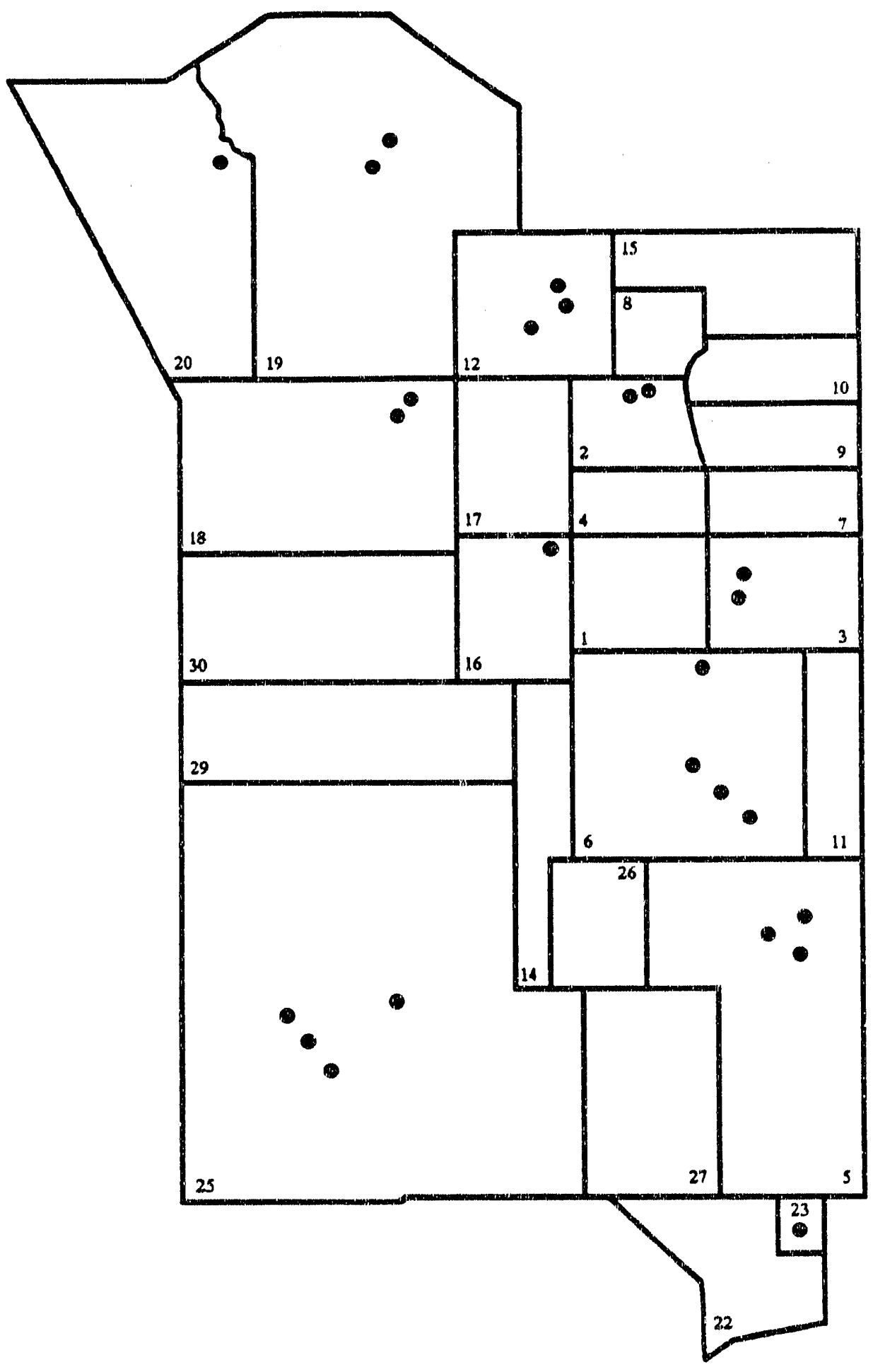

Figure 5.3. Man-made well and pond water sources at the Nevada est Site. 
Table 5.1 Approximate elevation and location of natural springs on the Nevada Test Site monitored for wildlife utilization.

\begin{tabular}{lcccc}
\hline Spring & Elevation (ft). & Area & \multicolumn{2}{c}{ Nevada Grid Coordinates } \\
\hline Cane & 4060 & 5 & N 746,300 & E 667,400 \\
Tippipah & 5200 & 16 & N 835,000 & E 635,100 \\
Topopah & 5820 & 29 & N 797,000 & E 616,300 \\
Reitman & 4600 & 7 & N 853,900 & E 702,300 \\
White Rock & 5050 & 12 & N 892,800 & E 655,700 \\
Captain Jack & 5880 & 12 & N 880,700 & E 645,050 \\
Oak & 5850 & 15 & N 909,000 & E 672,800 \\
Tub & 5230 & 15 & N 907,300 & E 681,700 \\
Gold Meadows & 6720 & 12 & N 902,900 & E 634,100 \\
Yucca Airstrip & 3900 & 6 & N 803,900 & E 681,000 \\
\hline
\end{tabular}


Table 5.2 Approximate elevation and location of well reservoirs on the Nevada Test Site monitored for wildlife utilization.

\begin{tabular}{|c|c|c|c|c|}
\hline \multirow{2}{*}{$\frac{\text { Reservoir }}{\text { Mercury Sewage }}$} & \multirow{2}{*}{$\frac{\text { Elevation }(\mathrm{ft})}{3620}$} & \multirow{2}{*}{$\frac{\text { Area }}{23}$} & \multicolumn{2}{|c|}{ Nevada Grid Coordinates } \\
\hline & & & N 694,400 & E 691,840 \\
\hline Well J11 & 3440 & 25 & N 740,880 & E 611,700 \\
\hline Well J12 & 3130 & 25 & $N 733,440$ & E 580,800 \\
\hline Well J13 & 3280 & 25 & N 750,800 & E 579,000 \\
\hline NUM AX & 3060 & 25 & N 725,000 & E 581,250 \\
\hline Well 5B & 3095 & 5 & N 747,440 & E 704,560 \\
\hline Well UE5C & 3210 & 5 & N 760,080 & E 701,000 \\
\hline Stream & 3130 & 5 & N 755,000 & E 704,600 \\
\hline Well C1 & 3920 & 6 & N 790,400 & E 692,000 \\
\hline CP Sewage & 4030 & 6 & N 794,800 & E 680,260 \\
\hline Well 3 & 3970 & 6 & N 818,000 & E 677,680 \\
\hline Well A & 4005 & 3 & $N 833,360$ & E 684,320 \\
\hline Mud Plant & 4025 & 3 & N 836,880 & E 686,000 \\
\hline Well 16D & 4680 & 16 & N 844,720 & E 646,800 \\
\hline Well 2 & 4480 & 2 & N 880,000 & E 669,040 \\
\hline Mud Plant & 4500 & 2 & N 878,800 & E 666,640 \\
\hline Sewage & 5100 & 12 & N 892,,500 & E 651,300 \\
\hline N Tunnel & 5750 & 12 & N 891,000 & E 641,000 \\
\hline Carnp 17 & 5760 & 18 & N 878,750 & E 618,750 \\
\hline Well 8 & 5700 & 18 & N 878,875 & E 609,200 \\
\hline Well U19C-Lower & 6680 & 19 & N 916,250 & E 600,000 \\
\hline Well U19C-Upper & 6900 & 19 & N 918,750 & E 602,500 \\
\hline Well $20 \mathrm{~A}$ & 6500 & 20 & $N 920,100$ & E 570,000 \\
\hline
\end{tabular}


Table 5.3 Total numbers of wildlife observed at natural springs at the Nevada Test Site during 1988. $P$ indicates utilization by a species inferred from animal sign.

*Approximate estimate only

\begin{tabular}{|c|c|c|c|c|c|c|c|c|c|c|c|}
\hline & 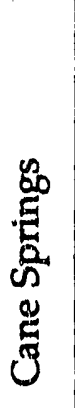 & 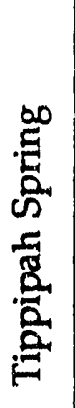 & 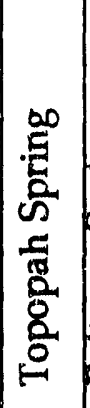 & 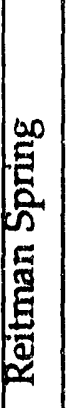 & 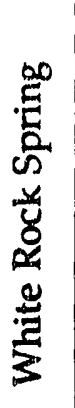 & 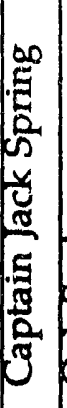 & 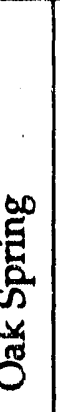 & 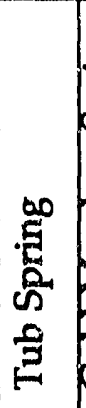 & 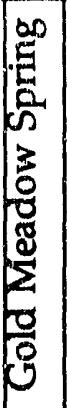 & 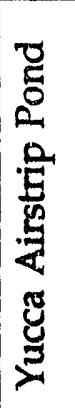 & $\stackrel{\text { శึ }}{\circ}$ \\
\hline Number of Visits & 5 & 6 & 4 & 5 & 6 & 8 & 2 & 6 & 5 & 4 & 51 \\
\hline Mule Deer & $P$ & $\mathbf{P}$ & P & $P$ & $P$ & 21 & $P$ & $P$ & 9 & $\mathrm{P}$ & 30 \\
\hline Feral Horse & & & & & & 24 & & & 1 & & 25 \\
\hline Mountain Lion & & & & & & & & & $P$ & & \\
\hline Bobcat & $P$ & & & & & & & & & & \\
\hline \multicolumn{12}{|l|}{ unt lope } \\
\hline Coyote & $\mathrm{P}$ & & & & $P$ & $P$ & & & $P$ & & \\
\hline \multicolumn{12}{|l|}{ Kit Fox } \\
\hline Cottontail Rabbit & & 1 & & & 8 & & & 1 & & & 10 \\
\hline Jack Rabbit & & & & & 1 & & 2 & & 1 & & 4 \\
\hline Chukar & & & $* 500$ & & & & & & & 24 & 524 \\
\hline Gambels Quail & 30 & 34 & 20 & 6 & 110 & & & 15 & & & 215 \\
\hline Mourning Dove & & 60 & & 10 & 5 & 1 & 1 & & & & 77 \\
\hline Buzzard Hawk (Buteo) & & & 1 & & 1 & 1 & & & & & 3 \\
\hline Great Blue Heron & & & & & & & & & & $P$ & \\
\hline \multicolumn{12}{|l|}{ White Faced Ibis } \\
\hline \multicolumn{12}{|l|}{ Belted Kingfisher } \\
\hline Common Raven & 2 & 1 & & & 3 & & & & 1 & & 7 \\
\hline \multicolumn{12}{|l|}{ Killdeer } \\
\hline Unidentified Ducks & & & & & & & & & & 43 & 43 \\
\hline Unidentified Owls & 1 & & & & & 1 & & & & 2 & 4 \\
\hline
\end{tabular}


Table 5.4 Total numbers of wildlife observed at well reservoirs on the Nevada Test Site during 1988. $P$ indicates utilization by a species inferred from animal sign.

\begin{tabular}{|c|c|c|c|c|c|c|c|c|c|c|c|c|c|c|c|c|c|c|c|c|c|}
\hline & 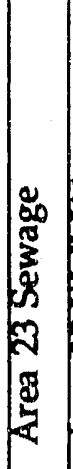 & 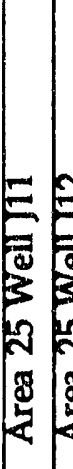 & 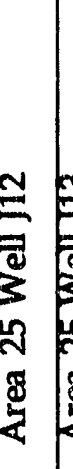 & 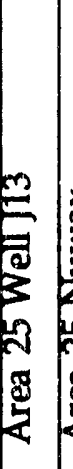 & 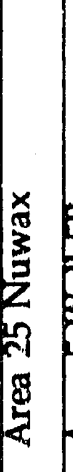 & 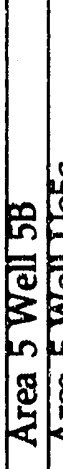 & 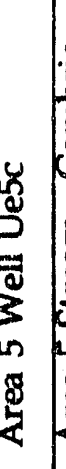 & 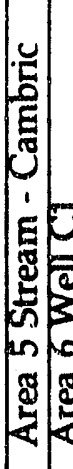 & $\begin{array}{l}3 \\
3 \\
3 \\
3 \\
0 \\
8\end{array}$ & 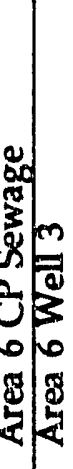 & एक & 至 & 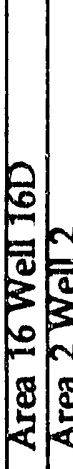 & 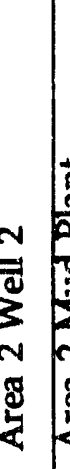 & 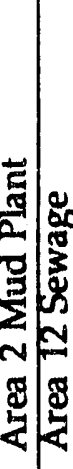 & 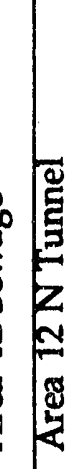 & 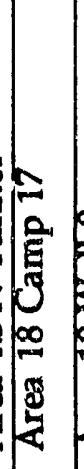 & 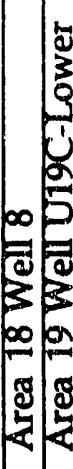 & 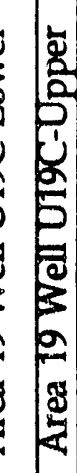 & 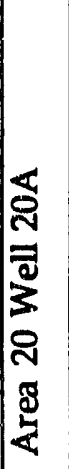 & $\frac{0}{00}$ \\
\hline Number of Visits & 6 & 3 & 3 & 3 & 3 & 9 & 8 & 5 & 4 & $\begin{array}{ll}3 & 6\end{array}$ & 2 & 4 & 6 & 3 & 3 & $1 \mid 1$ & 7 & 3 & $5 \longdiv { 2 }$ & 3 & 93 \\
\hline Mule Deer & & & & $P$ & & $P$ & $P$ & $P$ & & & & & $\mathrm{P}$ & & & \begin{tabular}{l|l}
$P$ & $P$ \\
\end{tabular} & $P$ & $\mathrm{P} 1$ & \begin{tabular}{l|l}
$\mathrm{P}$ & 1 \\
\end{tabular} & $\mathrm{P}$ & 1 \\
\hline Feral Horse & & & & & & & & & & & & & $P$ & & & $P$ & 65 & & $\mathrm{P}$ & $\bar{P}$ & 65 \\
\hline Mountain Lion & & & & & & & & & & & & & & & 1 & 1 & & & & & 1 \\
\hline Bobcat & & $\mathrm{P}$ & & & & $P$ & & & & & & & $\mathbf{P}$ & & & & & & & & \\
\hline Cattle & & & $P$ & $P$ & $\mathrm{P}$ & & & & & & & & & & & & $P$ & $\mathrm{P}$ & & & \\
\hline Coyote & $P$ & $P$ & $\mathrm{p}$ & $P$ & $P$ & $\mathrm{P}$ & $\mathrm{P}$ & $\mathrm{P}$ & PII & \begin{tabular}{l|l}
$\mathrm{P}$ & 1 \\
\end{tabular} & & $P$ & $\mathrm{P}$ & $P$ & 1 & $P$ & & & & & 2 \\
\hline Kit Fox & & & & & & & $\mathrm{P}$ & $\mathrm{P}$ & & $P$ & & & & & & & & & & & \\
\hline Cottontail Rabbit & & & 3 & & & 1 & & 1 & 2 & & & & & & 1 & & & & & & 8 \\
\hline Jack Rabbit & $\mathrm{P}$ & $P$ & 4 & $\mathrm{P}$ & $P$ & $\mathrm{P}$ & $P$ & $\mathrm{P}$ & & $P$ & & $P$ & & & & & & & & & 4 \\
\hline Chukar & & & & & & & & & & 6 & & & 10 & & & & & & 6 & & 22 \\
\hline Gambels Quail & 15 & & 12 & 90 & & & & 19 & 90 & & & & & & & 50 & 6 & & & & 318 \\
\hline Mourning Dove & 15 & & & 1 & 6 & 1 & & 1 & & & 1 & 17 & & 3 & & & 1 & 1 & 1 & & 38 \\
\hline Golden Eagle & & & & & & & 1 & & & & & & 1 & & & & & & & & 2 \\
\hline Northern Harrier & & & & & 2 & & & 5 & & & & & & & & & & & & & 7 \\
\hline Buzzard Hawk & 7 & 1 & 1 & & & 3 & 2 & 2 & & 1 & & & & & & & & & & & 17 \\
\hline Great Blue Heron & 2 & & 1 & & 1 & 1 & 2 & & 1 & 2 & & & & & & & 1 & & & & 11 \\
\hline White Faced lbis & 1 & & & & & & & & & & & & & & & & & & & & 1 \\
\hline Belted Kingfisher & & & & & & & & & & 1 & & & & & & & 1 & & & & 2 \\
\hline Common Raven & 64 & & 1 & & & 1 & 1 & 1 & & 29 & & 3 & 4 & 2 & 1 & & & & & & 110 \\
\hline Killdeer & 26 & & & & & & 1 & & & 2 & & & & & & 3 & & & 1 & & 32 \\
\hline Unidentified Ducks & 68 & & & & 3 & 11. & 50 & & & 2 & & & & & & & 2 & & & & 136 \\
\hline
\end{tabular}


.

)

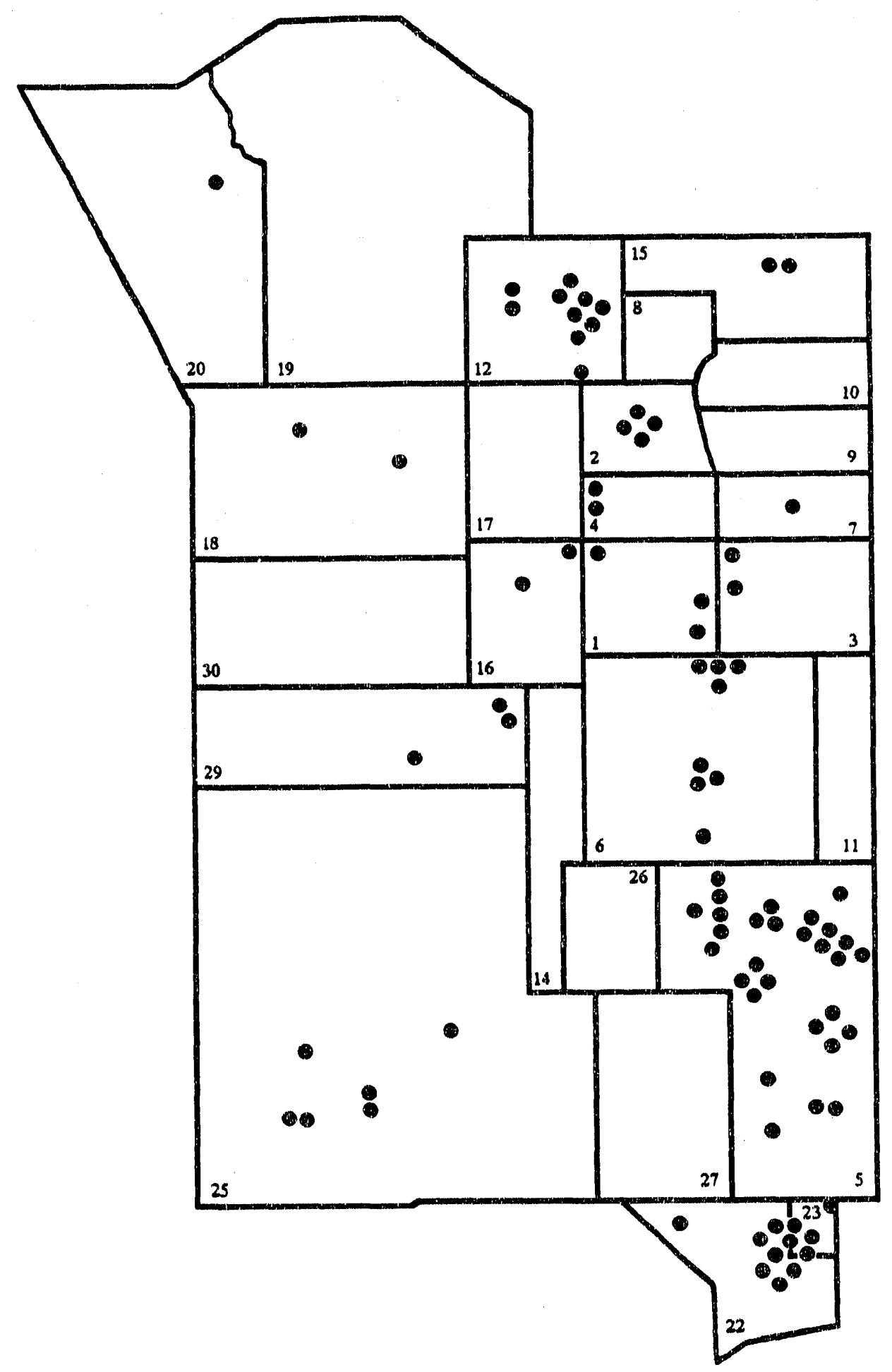

Figure 5.4 Sightings of individual raptors during $1988(n=87)$. 


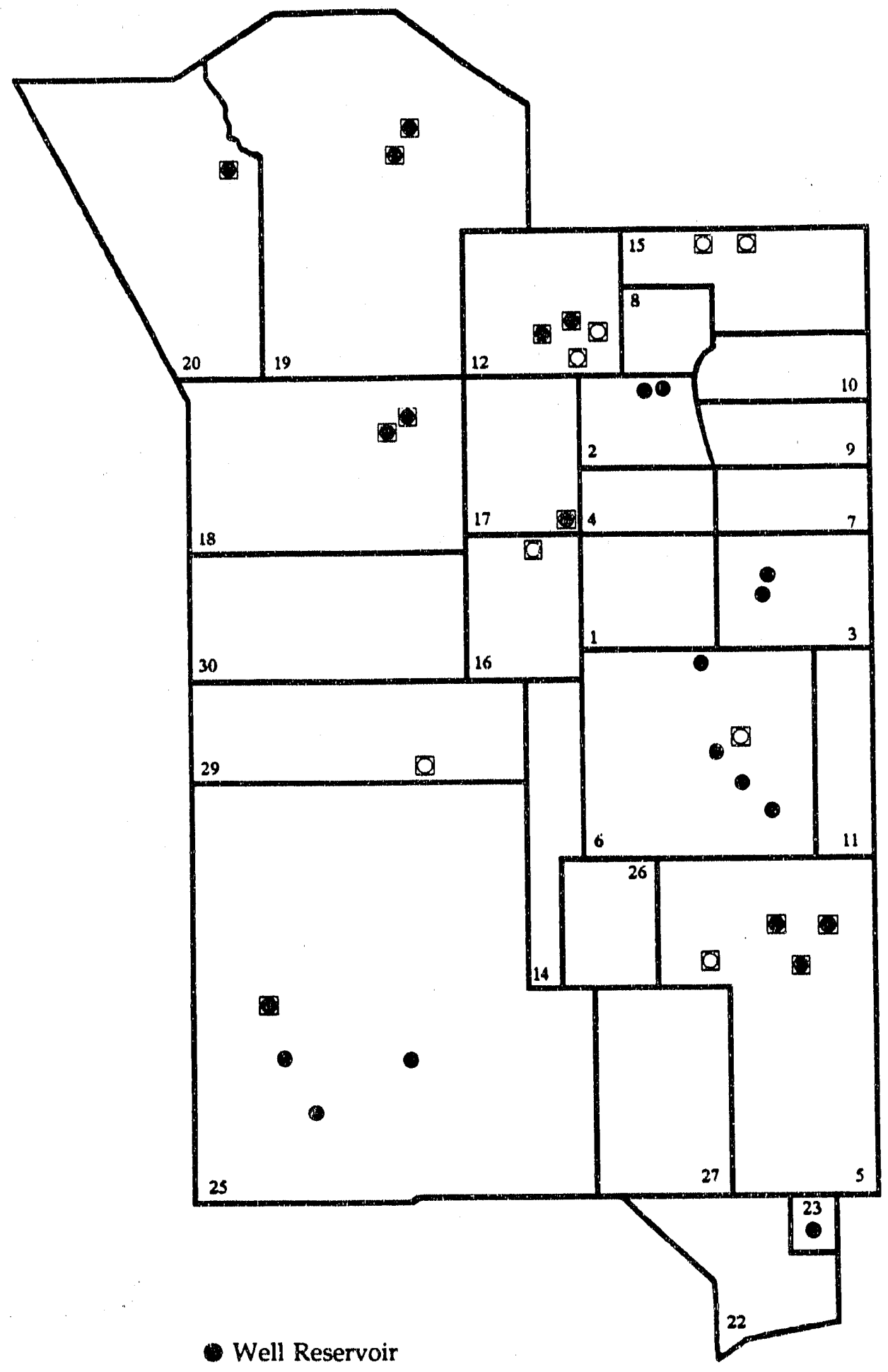

O Spring

$\square$ Mule Deer

Figure 5.5 Mule deer utilization of water sources at the Nevada Test Site. 


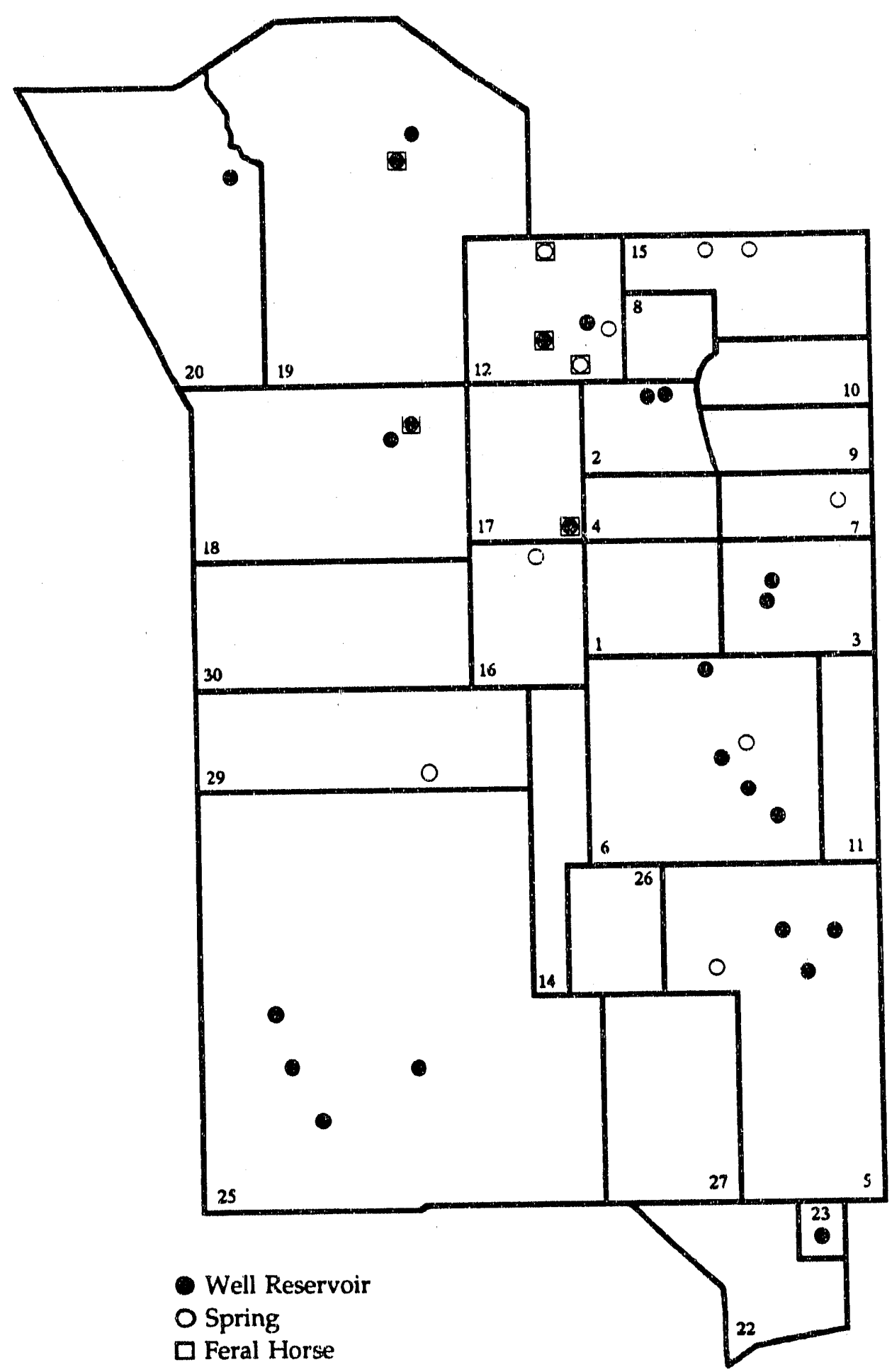

Figure 5.6 Feral horse utilization of water sources at the Nevada Test Site. 


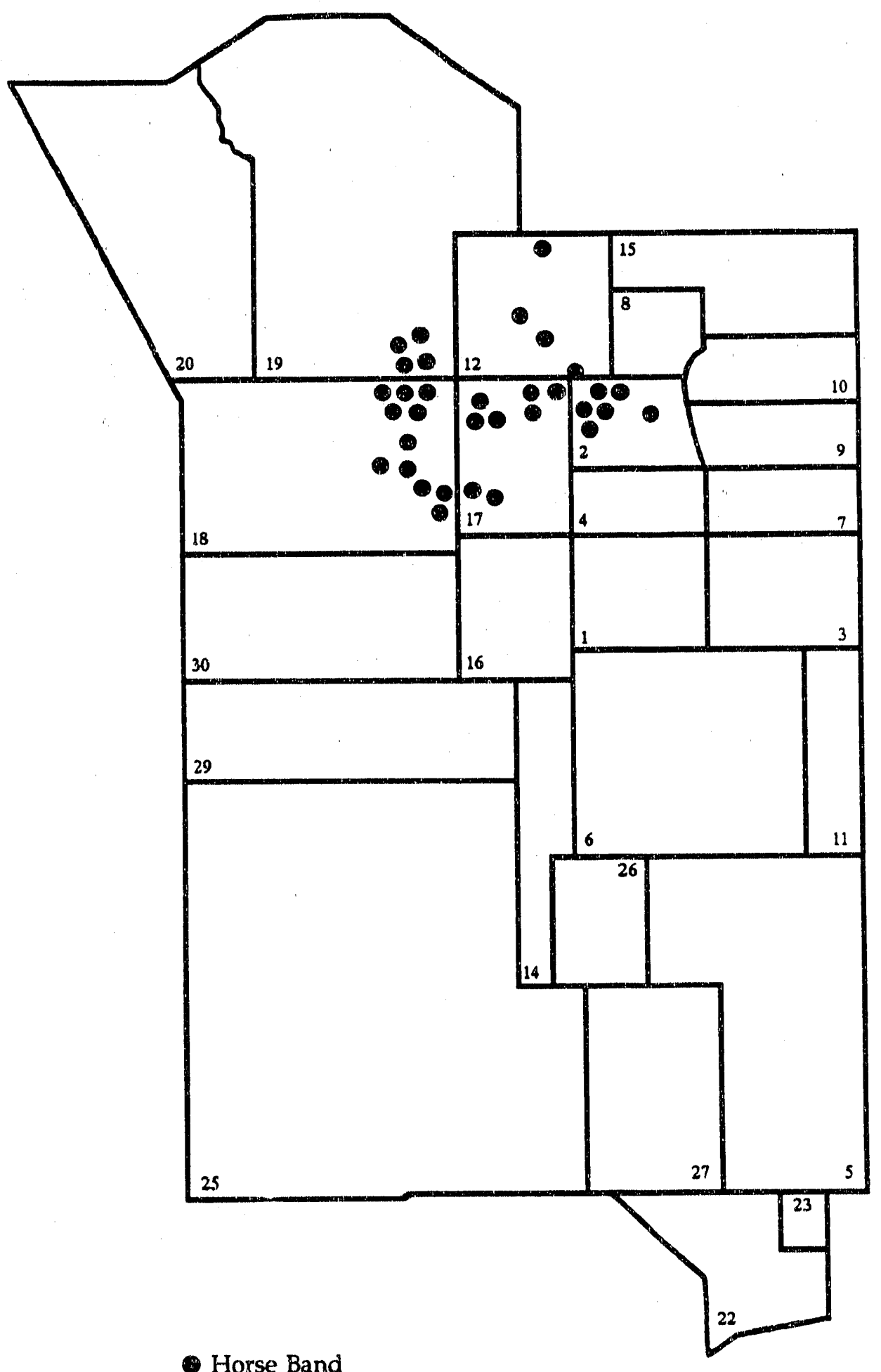

.

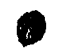

Horse Band

Figure 5.7 Feral horse sightings at the Nevada Test Site $\left(1_{*}=33\right)$. 


\section{DISCUSSION}

The first full year of monitoring activity has provided opportunity to learn more about the utilization of both natural spring and man-made water sources by wildlife: However, the findings are mainly qualitative and made primarily from secondary evidence such as the absence or presence of scats and tracks. We have had very few direct sightings of large animals at or near those water sources during daylight visits. Bird identification was significantly limited in 1988 because of the difficulty in obtaining a binocular permit. Our monitoring personnel do not have sufficient time to quantify the population size and nesting status of sensitive raptor species living on the NTS property. However, an investigator with appropriate training and experience resides in Las Vegas who could provide that need on a periodic consultant basis. Should information on raptors be needed beyond numbers that we can provide from opportunistic sightings, we recommend that this consultant-specialist be hired to obtain such information.

Most natural spring water sources at the NTS were improved by earlier ranchers to enhance utilization for livestock. Those improvements now have deteriorated so that access to water is generally poor as the result of clogged drain pipes and collapsed embankments. Because of present interest to discourage utilization of water sources by feral horses, we do not recommend improvement of any of the natural springs beyond the removal of reeds and trash, relic pipes and tanks, and other debris abandoned from ranching activity. Present condition of the natural spring water sources will allow them to continue serving the needs of dependent wildlife populations. Each of the man-made water sources investigated to date is being utilized by some form of wildlife, and all of the larger impoundments also are being used by migrating waterfowl. It is logical to assume that the presence of these man-made water sources has resulted in an increase in nearby populations of wildlife.

Daylight sighting of large game animals at or near the NTS water sources primarily involved mule deer. Numerous coyotes also have been observed. A small herd of antelope (ten to fifteen) was seen just before sunset in September 1988 in Area 20, and two different bull elk sightings were made in Area 18; one in August 1988, the other in October 1988. Future sightings of elk on the NTS may be expected as the result of recent establishment of herds on the Spring Mountain Range. Mountain lion tracks were observed in snow and mud around Gold Meadows Spring, and the evaporation pond at the sewage processing system near Area 12 camp. An adult mountain lion was also observed at this site for three consecutive days after it had killed a doe and returned to consume it each day.

The mineral element balance in both natural sping and well water supplies was surprisingly good at most locations on the NTS. The concentration level of given elements remains essentially the same throughout the year, except for some dilution effects resulting from short periods of snow melt and high rainfall runoff. It is interesting to note that, except for Reitman seep, the sodium content of other water sources at the NTS is less than that of 
tap water supplied to Mercury. The present treatment system for softening water for Mercury results in sodium levels in drinking water inappropriate for personnel on sodiumrestricted diet.

The present population of feral horses is estimated to be approximately fifty to sixty animals for the total NTS property, and they are restricted to a relatively small area as indicated from sighting data given in Figure 5.7. The present population appears to have sufficient forage, shelter and water to supply their needs. However, if their population increases, we may expect to see a broader foraging range. One group of nine feral horses was observed foraging in grassland on sites where earlier aboveground nuclear events were conducted in Area 2. Those grassland sites and others in northem Yucca Flat that have developed on disturbed land through natural succession of vegetation can be expected to be utilized by feral horses in the future should the herd size increase.

The most serious threat from feral horses in the future is the possibility that herds presently confined to the TTR proparty might move south onto the NTS property. We have considered at length why they have not already done so, and concluded that it is most likely because no watter sources are located within the forty-mile-wide stretch of land across the northern boundary of the NTS. Southern movement of TTR herds is further discouraged by the lack of good forage within that forty mile expanse of impoverished land. Nevertheless, the NTS management should take great care in permitting water sources to be developed across the northern and northwest boundary of the NTS and beyond. Otherwise, the gateway may be opened for intrusion of the TTR feral horses onto the NTS. Should it become necessary to extend water sources further north, adequate steps must be taken to make those sources unavailable to feral horses. 


\section{REFERENCES}

Allred, D. M., D E. Berk and C. D. Jorgensen. 1963. Biotic Communities of the Nevada Test Site. Brigham Young University, Sci. Bull. Biol. Ser. 2(2):1-52.

Alexander, G. V. and L. T. McAnulty. 1981. Multi-element analysis of plant-related tissues and fluids by optical emission spectrometry. J. Plant Nutr. 3:51-59.

Burt, W. H., and R. P. Grossenheider. 1976. A field guide to the mammals. Third Edition. Houghton Mifflin Company, Boston. 289 pp.

Giles, K. R. 1976. Springs at the Nevada Test Site and their use by wildlife. USEPA Report, NERC.LB.539-26. 14 pp.

Giles, K. R., and J. Cooper. 1985. Characteristics and migration patterns of mule deer on the Nevada Test Site. USEPA Report, EPA 600/4-85-030. 17 pp.

Hayward, C. L., M. L. Killpack and G. L. Richards. 1963. Birds of the Nevada Test Site. Brighain Young University, Sci. Bull., Biol. Ser. 3(1):1-27.

Jorgensen, C. D., and C. L. Hayward. 1965. Mammals of the Nevada Test Site. Brigham Young University, Sci. Bull., Biol. Ser. 6(3):1-81.

Murie, O. J. 1974. A field guide to animal tracks. Second Edition. Houghton Mifflin Company, Boston. 375 pp.

Peterson, R. T. 1961. A field guide to westem birds. Second Edition. Houghton Mifflin Company, Boston. 309 pp.

Robbins, C. S., et al. 1983. A guide to field identification of North American birds. Western Publishing Company, Inc., Racine, Michigan. 306 pp.

Taylor, W. D., and K. R. Giles. 1979. Freshwater algae of the Nevada Test Site. USEPA Report EMS6-LV-0539-25. 19 pp. 
Appendix 5

NTS NATURAL SPRINGS SAMPLES 1988

(Arranged by site and date, mg/liter)

\begin{tabular}{|c|c|c|c|c|c|c|}
\hline SAMPLE & $\mathrm{Na}$ & $\mathrm{K}$ & $\mathrm{Ca}$ & $\mathrm{Mg}$ & $\mathrm{P}$ & $\mathrm{Fe}$ \\
\hline Budweiser 3-17 & 45.5 & 113 & 23.7 & 6.73 & 14.1 & .110 \\
\hline Cane Sp 8.9 & 37.3 & 9.31 & 31.1 & 9.24 & 1.62 & .011 \\
\hline Cane Sp 10-31 & 39.3 & 11.6 & 33.3 & 9.40 & 1.05 & .003 \\
\hline Capt Jack 3 10 & 32.1 & 2.36 & 2.82 & .882 & .242 & 2.90 \\
\hline Capt Jack 8-1 & 38.8 & 4.83 & 2.20 & .771 & .540 & 2.59 \\
\hline Rock Val SP 1-25 & 2.18 & 0 & 17.0 & .554 & .749 & .011 \\
\hline Gold Med 6-21 & 6.10 & 12.3 & 18.8 & 1.72 & .723 & .023 \\
\hline Gold Med 10-28 & 7.18 & 26.2 & 24.5 & 25.80 & .807 & .011 \\
\hline Mercury Tap & 102 & 7.20 & 4.75 & 1.21 & 1.46 & .007 \\
\hline Oak Sp 11-17 & 74.2 & 41.6 & 111 & 17.7 & 3.80 & .113 \\
\hline Reitman 8-18 & 201 & 18.7 & 17.5 & 6.98 & 2.57 & 18.9 \\
\hline Reitman 11-16 & 153 & 20.4 & 11.8 & 1.27 & 1.48 & .529 \\
\hline Tippipah 3-4 & 37.6 & 3.12 & 4.22 & .747 & .258 & 1.69 \\
\hline Tippipah 11-01 & 42.3 & 7.05 & 11.3 & 1.10 & .923 & .456 \\
\hline Topopah 2-8 & 13.2 & 5.16 & 6.29 & 1.42 & .211 & .471 \\
\hline Topopah 7.27 & 12.5 & 11.1 & 7.98 & 1.84 & 1.39 & .346 \\
\hline Topopah 11-07 & 12.2 & 13.9 & 5.56 & 1.26 & .426 & .247 \\
\hline Tub Sp 4-2 & 30.5 & 3.44 & 15.7 & 2.70 & .875 & .006 \\
\hline Tub Sp 8-19 & 29.8 & 4.66 & 13.1 & 2.42 & 1.01 & .006 \\
\hline Tub, Cave 11-10 & 29.9 & 13.0 & 15.0 & 2.53 & .527 & .019 \\
\hline Tub, Out 11-10 & 30.9 & 13.7 & 15.7 & 2.60 & .664 & .232 \\
\hline White Rk 1-29 & 37.7 & 6.29 & 4.70 & 1.33 & 1.00 & 4.85 \\
\hline White Rk 3-4 & 38.9 & 6.75 & 4.87 & 1.10 & .725 & 4.11 \\
\hline White Rk 8-18 & 43.9 & 5.50 & 4.81 & .852 & .585 & 2.94 \\
\hline White Rk 11-10 & 43.1 & 18.4 & 9.22 & 2.76 & 1.34 & 9.86 \\
\hline
\end{tabular}


Appendix 5 (Continued)

NTS NATURAL SPRINGS SAMPLES 1988

(Arranged by site and date, $\mathrm{mg} / \mathrm{liter}$ )

\begin{tabular}{|c|c|c|c|c|c|c|}
\hline SAMPL.E & $\mathrm{Cr}$ & $\mathrm{Cu}$ & $\mathrm{Mn}$ & $\mathrm{Al}$ & $\mathrm{Cd}$ & $\mathrm{Pb}$ \\
\hline Budweiser 3-17 & 0 & .145 & .002 & .236 & 0 & 0 \\
\hline Cane Sp 8-9 & .006 & 0 & .001 & .379 & .005 & .025 \\
\hline Cane Sp 10-31 & 0 & 0 & 0 & .161 & .019 & .006 \\
\hline Capt Jack 3-10 & .002 & .011 & .023 & 7.37 & 0 & 0 \\
\hline Capt Jack 8-1 & .012 & .004 & .024 & 7.45 & .052 & .048 \\
\hline Rock Val Sp 1-25 & .001 & .003 & .000 & .118 & .010 & .015 \\
\hline Gold Med 6-21 & .007 & .009 & .002 & .285 & .022 & .042 \\
\hline Gold Med $10-28$ & 0 & .001 & .000 & .182 & .025 & .027 \\
\hline Mercury Tap & .004 & .036 & 0 & .090 & .217 & 0 \\
\hline Oak Sp 11-17 & 0 & 0 & 3.21 & .368 & .057 & .113 \\
\hline Reitman 8-18 & .009 & .010 & .186 & 35.9 & .019 & .065 \\
\hline Reitman 11-16 & .002 & .004 & .090 & .412 & .060 & .053 \\
\hline Tippipah 3-4 & .002 & .003 & .022 & 4.00 & 0 & .007 \\
\hline Tippipah 11-01 & .003 & .000 & .006 & 1.04 & .012 & .017 \\
\hline Topopah 2-8 & .004 & 0 & .005 & 1.53 & 0 & 0 \\
\hline Topo: ah 7-27 & .009 & .011 & .007 & 1.46 & .078 & .015 \\
\hline Topopah 11-07 & 0 & .003 & .002 & .732 & .021 & 0 \\
\hline Tub Sp 4-2 & .008 & .005 & .001 & .304 & .021 & 0 \\
\hline Tub sp 8-19 & .006 & .001 & .000 & .275 & .013 & .009 \\
\hline Tub, Cave 11-10 & .002 & 0 & 0 & .121 & .025 & 0 \\
\hline Tub, Out $11-10$ & .000 & .000 & .001 & .133 & .052 & 0 \\
\hline White Rk 1-29 & .005 & .002 & .073 & 10.8 & 0 & 0 \\
\hline White Rk 3-4 & 0 & 0 & .057 & 9.47 & 0 & 0 \\
\hline White Rk 8-18 & .006 & .004 & .033 & 7.20 & .009 & .019 \\
\hline White Rk $11-10$ & .005 & .007 & .128 & 20.7 & .019 & .015 \\
\hline
\end{tabular}


Appendix 5 (Continued)

NTS NATURAL SPRINGS SAMPLES 1988

(Arranged by site and date, $\mathrm{mg} / \mathrm{liter}$ )

\begin{tabular}{|c|c|c|c|c|c|c|}
\hline SAMPLE & $\mathrm{Ni}$ & $\mathrm{Be}$ & V & $\mathrm{Ti}$ & $\mathrm{Zn}$ & $\mathrm{Ag}$ \\
\hline Budweiser 3-17 & .024 & 0 & .012 & .005 & .238 & 0 \\
\hline Cane Sp 8-9 & .012 & .001 & .021 & 0 & .031 & .107 \\
\hline Cane Sp 10-31 & .009 & .000 & .015 & .000 & .031 & .117 \\
\hline Capt Jack 3-10 & 0 & .004 & .012 & .167 & .024 & .004 \\
\hline Capt Jack 8-1 & .022 & .001 & .018 & .158 & .037 & .006 \\
\hline Rock Val Sp 1-25 & 0 & .001 & .004 & .001 & .000 & .031 \\
\hline Gold Med 6-21 & .021 & .003 & .015 & .001 & .008 & .154 \\
\hline Gold Med 10-28 & 0 & .002 & .006 & 0 & .007 & .246 \\
\hline Mercury Tap & .001 & .001 & .021 & 0 & .030 & .025 \\
\hline Oak Sp 11-17 & .026 & .000 & .005 & 0 & .054 & .077 \\
\hline Reitman 8-18 & .008 & .003 & .036 & 1.02 & .106 & .025 \\
\hline Reitman 11-16 & 0 & 0 & .015 & .030 & .040 & .012 \\
\hline Tippipah 3-4 & 0 & .000 & .007 & .082 & .017 & .006 \\
\hline Tippipah 11-01 & 0 & 0 & .007 & .021 & .022 & .025 \\
\hline Topopah 2-8 & .003 & 0 & .007 & .021 & .007 & .007 \\
\hline Topopah 7-27 & .017 & .003 & .015 & .016 & .010 & .014 \\
\hline Topopah 11.07 & .008 & 0 & .005 & .011 & .006 & .008 \\
\hline Tub Sp 4-2 & 0 & .001 & .014 & .000 & .022 & .011 \\
\hline Tub Sp 8-19 & .011 & .000 & .009 & .000 & .016 & .009 \\
\hline Tub, Cave $11-10$ & 0 & 0 & .005 & .001 & .017 & .010 \\
\hline Tub, Out $11-10$ & 0 & 0 & .003 & .001 & .018 & .012 \\
\hline White Rk 1-29 & 0 & .001 & .016 & .280 & .029 & .004 \\
\hline White Rk 3-4 & 0 & .001 & .014 & .242 & .029 & .004 \\
\hline White Rk 8-18 & .010 & .002 & .015 & .177 & .029 & .004 \\
\hline White Rk 11-10 & 0 & .001 & .027 & .562 & .036 & .008 \\
\hline
\end{tabular}


Appendix 5 (Continued)

NTS NATURAL SPRINGS SAMPLES 1988

(Arranged by site and date, $\mathrm{mg} / \mathrm{liter}$ )

\begin{tabular}{|c|c|c|c|c|c|c|c|}
\hline SAMPLE & Mo & $\mathrm{Ba}$ & $\mathrm{Li}$ & $\mathrm{Sr}$ & As & Se & $\mathrm{Sn}$ \\
\hline Budweiser 3-17 & .082 & .307 & .051 & .141 & .058 & .019 & .090 \\
\hline Cane Sp 8-9 & .089 & 0 & .227 & .003 & 0 & .219 & .009 \\
\hline Cane Sp 10-31 & .058 & 0 & .106 & .009 & 0 & .030 & .023 \\
\hline Capt Jack 3-10 & .048 & 0 & .185 & .033 & 0 & .049 & .011 \\
\hline Capt Jack 8-1 & .031 & .096 & .246 & .028 & .004 & .218 & .000 \\
\hline Rock Val Sp 1-25 & .033 & 0 & .070 & .002 & 0 & .056 & .002 \\
\hline Gold Med 6-21 & .100 & .048 & .186 & .006 & .001 & .214 & .007 \\
\hline Gold Med 10-28 & .097 & .029 & .097 & .007 & 0 & .037 & .007 \\
\hline Mercury Tap & .044 & .026 & 0 & .010 & 0 & .151 & .001 \\
\hline Oak Sp 11-17 & .080 & 0 & .108 & 15.9 & 0 & .068 & .008 \\
\hline Reitman 8-18 & .093 & 0 & .175 & .172 & .003 & .012 & .025 \\
\hline Reitman 11-16 & .113 & 0 & .068 & .004 & 0 & .047 & .003 \\
\hline Tippipah 3-4 & 0 & 0 & .130 & .021 & .000 & .053 & .001 \\
\hline Tippipah 11-01 & .097 & .010 & .052 & .005 & .001 & .037 & .001 \\
\hline Topopah 2-8 & .037 & 0 & .216 & .007 & 0 & .042 & 0 \\
\hline Topopah 7-27 & .035 & .023 & .212 & .012 & .002 & .233 & 0 \\
\hline Topopah 11-07 & .055 & .002 & .026 & .015 & .002 & .020 & .002 \\
\hline Tub Sp 4-2 & .090 & 0 & .163 & .003 & .001 & .194 & .005 \\
\hline Tub Sp 8-19 & .103 & .023 & 0 & .001 & .002 & .049 & .004 \\
\hline Tub, Cave 11-10 & .036 & .032 & .049 & 0 & 0 & .038 & .002 \\
\hline Tub, Out 11-10 & .085 & 0 & .044 & 0 & .001 & .057 & .002 \\
\hline White Rk 1-29 & 0 & .048 & .211 & .041 & 0 & .047 & .008 \\
\hline White R.k $3-4$ & .032 & 0 & .144 & .039 & 0 & .050 & .008 \\
\hline White R.k 8-18 & .039 & .008 & .072 & .027 & .004 & .038 & .007 \\
\hline White Rk 11-10 & .122 & .045 & .131 & .081 & .001 & .021 & .011 \\
\hline
\end{tabular}


2 


\title{
SECTION 6 STATUS OF PERENNIAL PLANTS ON THE NEVADA TEST SITE IN 1988
}

\author{
by \\ Richard B. Hunter
}

\section{INTRODUCTION}

Perennial plants are the major producers of biomass on the Nevada Test Site (NTS). They normally cover 20 to $30 \%$ of the surface at lower elevations, and 40 to $50 \%$ on the mesas (Beatley 1979), providing food and cover to the desert animals. They are ubiquitous except on the dry lake beds (playas) and newly disturbed areas. Most habitats support long-lived desert shrubs, mixed with sparse short-lived herbaceous perennials and winter ephemerals. The higher altitudes also support juniper trees (Juniperus osteosperma) and/or juniper mixed with pinyon pines (Pinus monophylla).

The shrub vegetation on the NTS has been studied since 1958 (reviewed in Hunter and Medica 1989). Changes in the populations are relatively slow (Beatley 1976), on the scale of human lifespans. Recovery of the vegetation following disturbance is also slow (Webb and Wilshire 1980; Lathrop and Archbold 1980), and recovery to an equilibrium status may never occur, given the documented long-term fluctuations in the world's climate since the last iceage (see for example Van Devender and Spaulding 1979). As such, the plant community is "fragile", though the individuals comprising it are adept at survival in a very harsh environment.

There are about 436 perennial plant species and subspecies inhabiting the NTS. By far the majority are uncommon, leaving a few species to dominate the vegetation in most areas. The dominant species (Table 6.1) are uniformly long-lived (decades to centuries) and very drought-tolerant. They do not invade disturbed areas rapidly, and those species which do are often absent or poorly represented in undisturbed habitats. Natural causes of mortality in mature plants are gophers, windfall, self-shading problems associated with old age or large size, fires and floods, drought, and insects.

The Basic Environmental Compliance and Monitoring Program (BECAMP) was established in 1986 by the Department of Energy Nevada Field Office (DOE/NV) to assess changes over time in the radiological and exological condition of the NTS and to provide information needed for NTS compliance with environmental laws. The ecological studies are intended to 
record the spatial

distributions of the flora

and fauna and their

changes with time on the

NTS. The purpose of the

perennial plant

measurements is to

determine population

densities and plant sizes

at particular locations, and to monitor changes in those variables by repeated measurements at intervals of one to five years. Because the perennial populations change slowly, monitoring changes is considered a long-term undertaking, requiring the maintenance of permanently marked sample populations and extended maintenance of records on individual plants for long periods. Nineteen-eighty-eight was the second year of sampling of perennial plants for the BECAMP program, and thus the first year for comparison of data between years. The resulting change in analytical procedures was still in experimental stages, but allowed an enhanced assessment of the changes occurring in the desert shrub communities sampled.

Areas sampled in 1988 included five baseline sites, three ground zeros from 1950s bomb tests, the scraped edge (verge) of an abandoned road, and a newly scraped site.

\section{METHODS}

Methods of shrub measurement changed slightly from those used in 1987 (Hunter and Medica 1989). The techniques used involved selecting a site, laying out a $50-\mathrm{m}$ steel surveyor's tape, and measuring all the perennial plants within one meter on both sides of the tape. In 1988 dead shrubs were measured as well as live ones, and the data sheets were modified slightly (Figure 6.1). (Dead grasses were recorded only when those measuring felt they were sparse enough to relocate individuals at a later time. Recorded locations were inadequate to distinguish among several close individuals of a given species.) In addition, the dead parts of live shrubs were estimated and given a code $0=$ no dead parts, $1=<25 \%$, $2=25-75 \%, 3=75-99 \%, 4=100 \%$ dead), whereas in 1987 the absolute percent dead was 


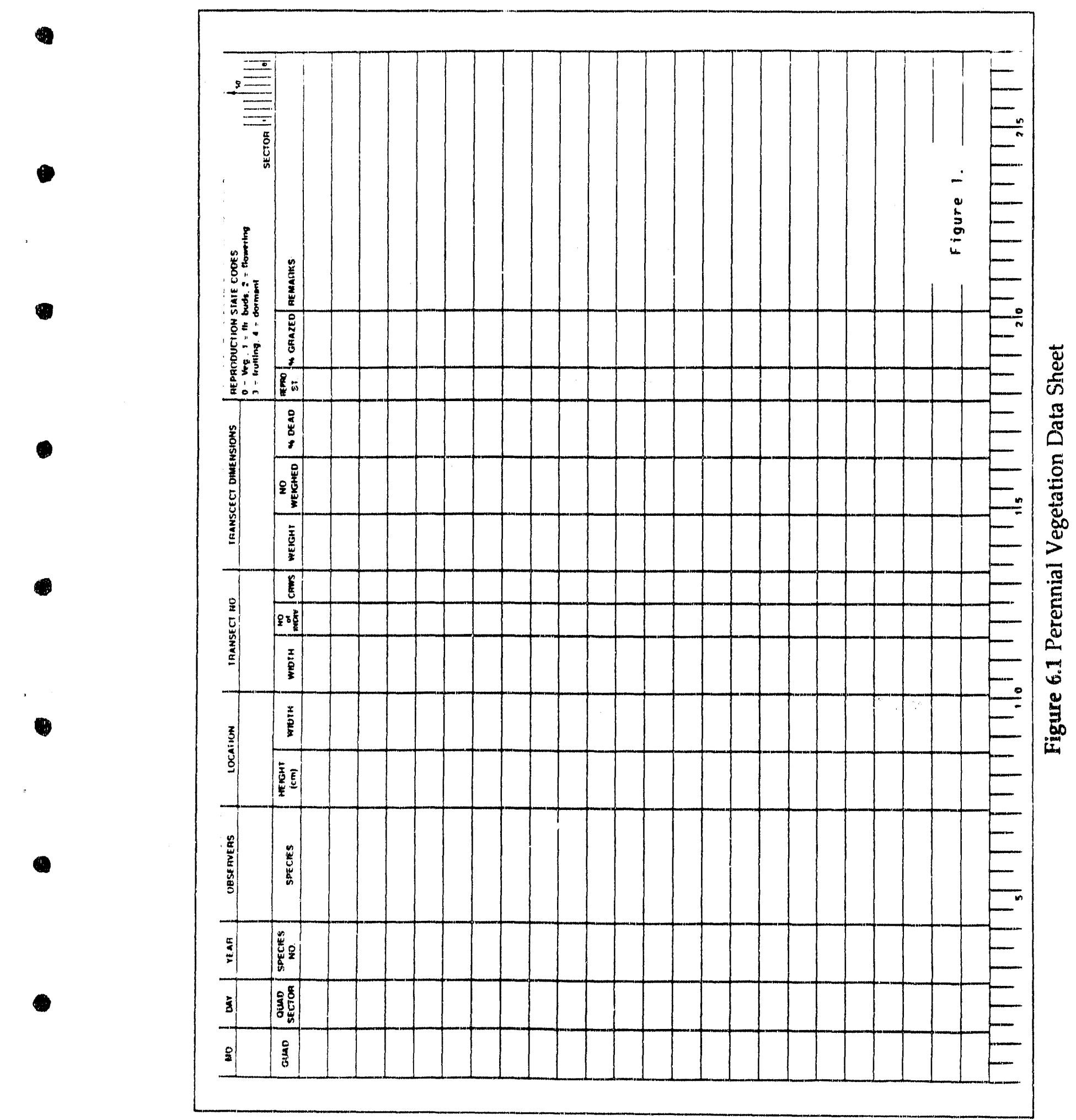


estimated (0-100\%). The system of recording distances of shrub bases from the tape was standardized, after experimenting with several techniques in 1987. In 1988 a "sector" was recorded, in which the two-meter width of the transect was divided into eight $0.25-\mathrm{m}$ sections, the first one being to the left as the person measuring faced the end $(50 \mathrm{~m})$ of the transect from any location along the transect (see diagram in Figure 6.1). Cover was calculated from width measurements on the shrubs, as in 1987. Note that the cover calculations were not corrected for overlapping canopies.

The locations of study sites are shown on the map (Figure 6.2), and their coordinates are given in Appendix 3A (page 83). Plant species names and their abbreviations are in Table 6.2, with nomenclature following Kartesz and Kartesz, 1980, with the exception of Haplopappus nanus, which follows Welsh et al. 1987.

Analyses of the data involved calculating a "live volume" for each plant measured, and using linear regression of size versus weight to estimate biomass of individual plants (Hunter and Medica 1989). In the absence of a volume/biomass regression line for Pinus monophylla for NTS populations (Hurter and Medica 1989), the biomass of that species was estimated from a published relationship for northern Nevada (Tausch and Tueller 1990). Only live portions of living plants were measured, but the whole extent of a dead individual was measured. Transects which were measured a second time in 1988 had the data from individual plants matched for the two years, and growth or shrinkage was calculated on the matched plants. Unmatched plants on the borders (sectors 1 and 8) of the 1988 transect which were large enough to have been easily seen in $1987(>20 \mathrm{~cm}$ in one dimension, and not under another shrub) were deleted from the 1988 data, as they were considered to be o1"tside the transect. A dead plant was considered to have a live volume, cover, and biomass of zero. Summary data were calculated both for populations and for individuals for those transects measured at two different times.

On several transects in Jackass Flats and Frenchmen Flat Larrea tridentata and Mendora spinescens were measured when they occurred within $2 \mathrm{~m}$ of the tape, instead of within $1 \mathrm{~m}$. The goal was to increase the numbers being monitored for those lower-density species. All other species on those transects were measured within $1 \mathrm{~m}$ of the tape.

Other parameters (height, maximum and perpendicular widths, reproductive states, etc.) were measured as in 1987 (Hunter and Medica 1989). Statistics were performed with the "RS1" program, Release 4 (BBN Software Products Corporation, Cambridge, MA). 


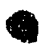

9

-

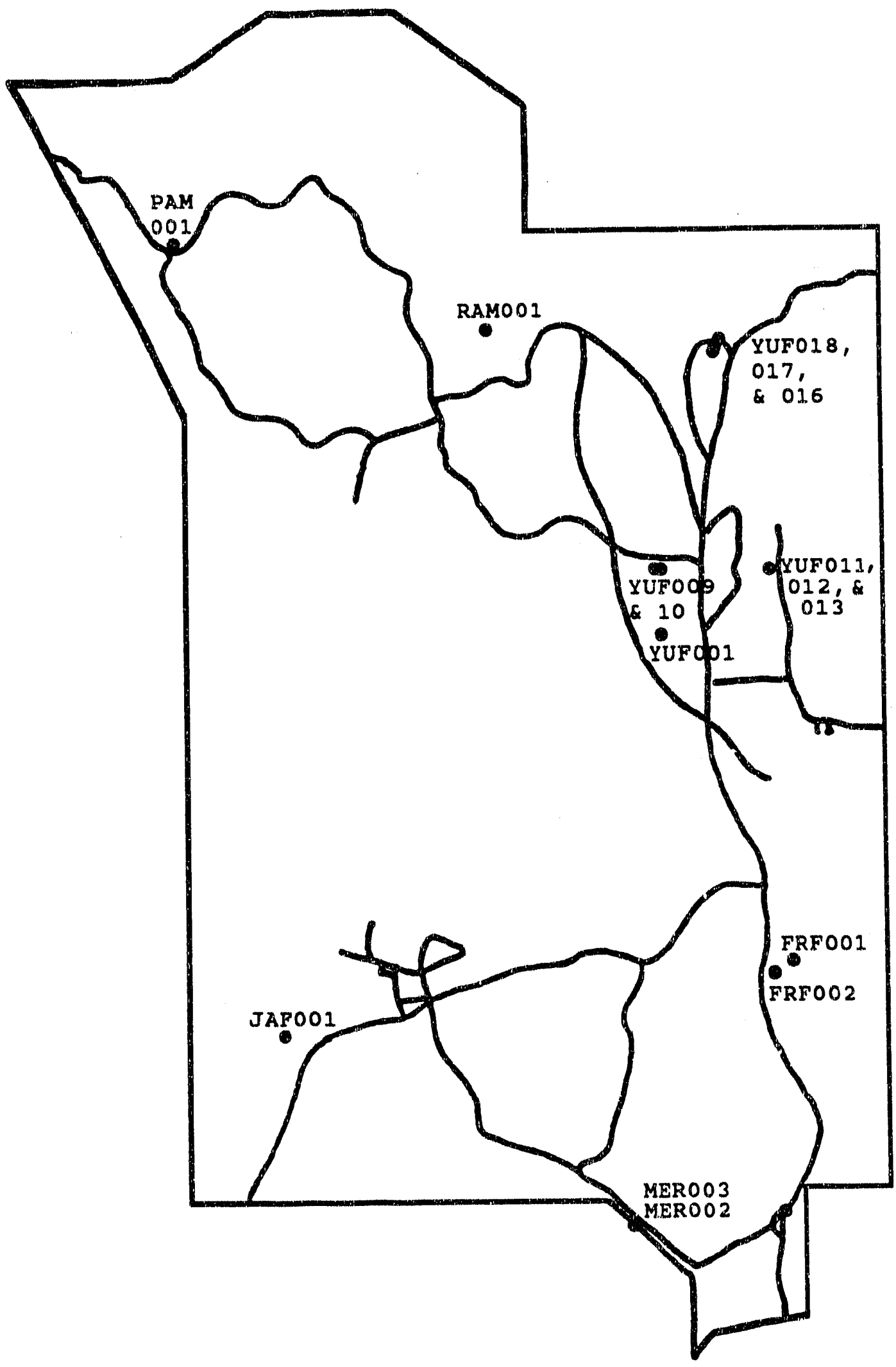

Figure 6.2 BECAMP study plots sampled for perennial plants in 1988 . 
Table 6.2 Perennial plant species names, authorities, and the abbreviations used in this report.

Abbreviation

Acamptopappus shockleyi Gray

Ambrosia dumosa (Gray) Payne

Arabis pulchra M.E. Jones ex S. Wats.

Arenaria congesta Nult. ex Torr. \& Gray

Artemisia nova A. Nels.

Artemisia spinescens D.C. Eat.

Artemisia tridentata Nutt.

Atriplex canescens (Pursh) Nutt.

Ceratoides lanata (Pursh) I.T. Howell

Chaenactis douglassii (Hook.) Hook. \& Arn.

Chrysothamnus nauseosus (Pallas) Britt.

Chrysothamnus viscidiflorus (Honk.) Nutt.

Coleogyne ramosissima Torr.

Cowania mexicana D. Don

Cryptantha flavoculata (A. Nels.) Payson

Dead grass

Dead shrub

Ephedra nevadersis S. Wats.

Eriogonum umbellatum TorT.

Erioneuron pulchellum (H.B.K.) Tateoka

Grayia spinosa (Hook.) Mog.

Gutierrezia sarothrae (Pursh) Britt. \& Rusby

Haplopappus nanus (Nutt.) D. C. Eat.

Hymenoclea salsola Torr. \& Gray ex Gray

Juniperus osteosperma (Torr.) Little

Kochia americana Benth.

Larrea tridentata (Sesse \& Moc. ex DC.) Coville

Linonthus nuttallii (Gray) Greene ex Milliken

Lycium andersonii Gray

Menodora spinescens Gray

Mirabilis pudica Barneby

Opuntia basilaris Engelm. \& Bigelow

Opuntia erinacea var. ursina (A. Weber) Parish

Oryzopsis hymenoides (Roemer \& Schultes) Ricker

Penstemon species

Pinus monophylla Torr. \& Frem.

Poa sandbergii Vasey

Polygala subspinosa S. Wats.

Quercus gambelii Nutt.

Sitarion jubatum J.G. Sm.

Sphaeralcea ambigua Gray

Stanleya pinnata (Pursh) Britt.

Stipa speciosa Trin. \& Rupr.

Streptanthus cordatus Nutt. ex Torr. \& Gray

Tetradymin axillaris A. Nels.

ACA SHO

AMB DUM

ARA PUL

ARE CON

ART NOV

ART SPI

ART TRI

ATR CAN

CER LAN

CHA DOU

CHR NAU

CHR VIS

COL RAM

COW MEX

CRY FLA

DEAD GS

DEAD SH

EPH NEV

ERI UMB

ERI PUL

CRA SPI

GUT SAR

HAP NAN

HYM SAL

JUN OST

KOC AME

LAR TRI

LIN NUT

LYC AND

MEN SPI

MIR PUD

OPU BAS

OPU EIRI

ORY HYM

PEN opp

PIN MON

POA SAN

POL, SUB

QUE GAM

SIT JUB

SPH AMB

STA PIN

STI SPE

STR COR

TET AXI

Tetradymia glabrata Torr. \& Gray

TET GLA

Yucca brevifolia Engelm.

YUC BRE

Yucca schidigera Roezl ex Ortgies

YUC SCH 


\section{RESULTS}

\section{MEASUREMENTS AT PREVIOUGLY SAMPLED LOCATIONS}

The baseline plots in Jackass Flats, Frenchman Flat, and Yucca Flat each had five transects measured in both 1987 and 1988, to give 15 transects measured both years. The task of matching plants between years was found to be both difficult and time consuming, and the quality of the 1987 data was found to be variable, depending on the person measuring the plants. For these reasons only a subset was analyzed as paired 1987-1988 data sets. "This included five of the fifteen transects, and one censused in 1986 for another project.

The new technique of matching plants between censuses made it possible to analyze sources of error in the plant transect data to a greater degree than previously possible. Table 6.3 shows the results of looking at the plants on trarisect JAFO01 which were not matchable at the two censuses. The most common cause of failure to match individual plants appeared to be a difference in criteria for including or excluding borderline plants between the two people doing the transect, and this was partially corrected by deleting selected 1988 plants as described in the Methods section. It appeared that in 1988 borderline plants were included which were excluded in 1987. In addition, the person measuring in 1988 appears to have searched mure carefully in clumps to measure the small hidden plants within them. The "ambiguous" plants were probably the same individuals each year. Ambiguities resulted sometimes from a subjective determination of whether a plant had two crowns or was two individuals, and sometimes from when two or more similar plants were near enough that their locations could not be distinguished with the data collected. On the whole, within the 100 square meters measured in 1987, 24 more plants were found in 1988 than in 1987. That was an increase of $9 \%$, which could be explained by the borderline changes and the better inclusion of small plants in clumps. (Inclusion of Larrea tridentata and Menodora spinescens in the extra 100 square meters sampled in 1988 also increased the total number sampled. Those plants were not included in Table 6.3.)

Adding $100 \mathrm{~m}^{2}$ to the area measured for Larrea tridentata and Menodora spinescens did not increase the number of measured plants enough to significantly increase their population sizes. The added area did make it more difficult to analyze and present the clata. The added plants were therefore not included in the analyses for any transect. This technique was subsequently abandoned.

Results for the six transects with matching data from previous years are in Tables 6.4-6.9. These data are from three "baseline" plots in Jackass Flats, Frenchman Flat, and Yucca Flat, and from one control transect at Waste Management Consolidation Site 3b, in Yucca Flat, which had been measured in 1986 prior to startup of the BECAMP program.

Tables 6.4 and 6.5 show perennial plant population characteristics on plot JAF001, which has a plant population dominated by Ambrosia dumosa, Acamptopappus shockleyi, and Larrea 
Table 6.3 Analysis of unmatched plants from 1987 and 1988 censuses on transect JAF001V1. Missed plants in 1988 were present in 1987, and new 1988 plants were presumably missed in 1987.

\begin{tabular}{lcc} 
& $\begin{array}{c}\text { Number Missed } \\
\text { in } 1988\end{array}$ & $\begin{array}{c}\text { Number New } \\
\text { in 1988 }\end{array}$ \\
\cline { 2 - 3 } & -15 & +13 \\
Ambiguous Crowns vs. Individuals & -4 & +20 \\
Borderline Plants & & +8 \\
Ambiguous Plants Close to & -12 & +14 \\
Similar Individuals & -5 & +4 \\
Small Plants Hidden in Clumps & -4 & +6 \\
Mismeasured or Misrecorded & -1 & +6 \\
Small Plants Overlooked & -1 & +4 \\
Plants Inside Menodora Canopies & -3 & +3 \\
Easily Visible but not Measured & & +0 \\
Plants Masked in Clumps with & -4 & +1 \\
Similar-Appearing Individuals & -4 & +78 \\
Died or Germinated 1987-1988 & -1 & \\
Misidentified Species & -54 & \\
\multicolumn{1}{c}{ Totals } & & +6 \\
\hline
\end{tabular}

tridentata. The plot is situated on a deep sandy soil near Forty Mile Canyon, at an altitude of 954 meters. This is one of the lowest areas on the NTS, and the plants were uniformly drought hardy and tolerant of high temperatures. The Larrea tridentata plants were old, having numerous "clonal" bases (crowns) within a clump of plants. They were relatively short, however, and had many dead branches.

Most of the differences seen between population characteristics in 1988 and 1987 probably reflect differences in measurement techniques of different individuals. Differences in plant numbers between the two years were attributable to the factors listed in Table 6.3, and to differences in the perception of Larrea tridentata. In 1987 each of several Larrea "crowns" was considered to be a separate plant, whereas in 1988 a group of crowns was considered to be a clonal plant. L. tridentata has been shown to have a clonal growth habit (Stemberg 1976), and we consider the differences to be a result of insufficient training for the individuals measuring during 1987. That change resulted in an apparent, but not real, decrease in number and increase in the average volume of L. tridentata plants, and accounted for most of the difference in total live plant cover.

As can be seen from the dead plant data (measured in 1988 only), dead plants accounted for only a small percentage of the population. The similarities in average plant sizes (Tables 6.4 and 6.5) aiso argued against germination events, which would have greatly increased plant 


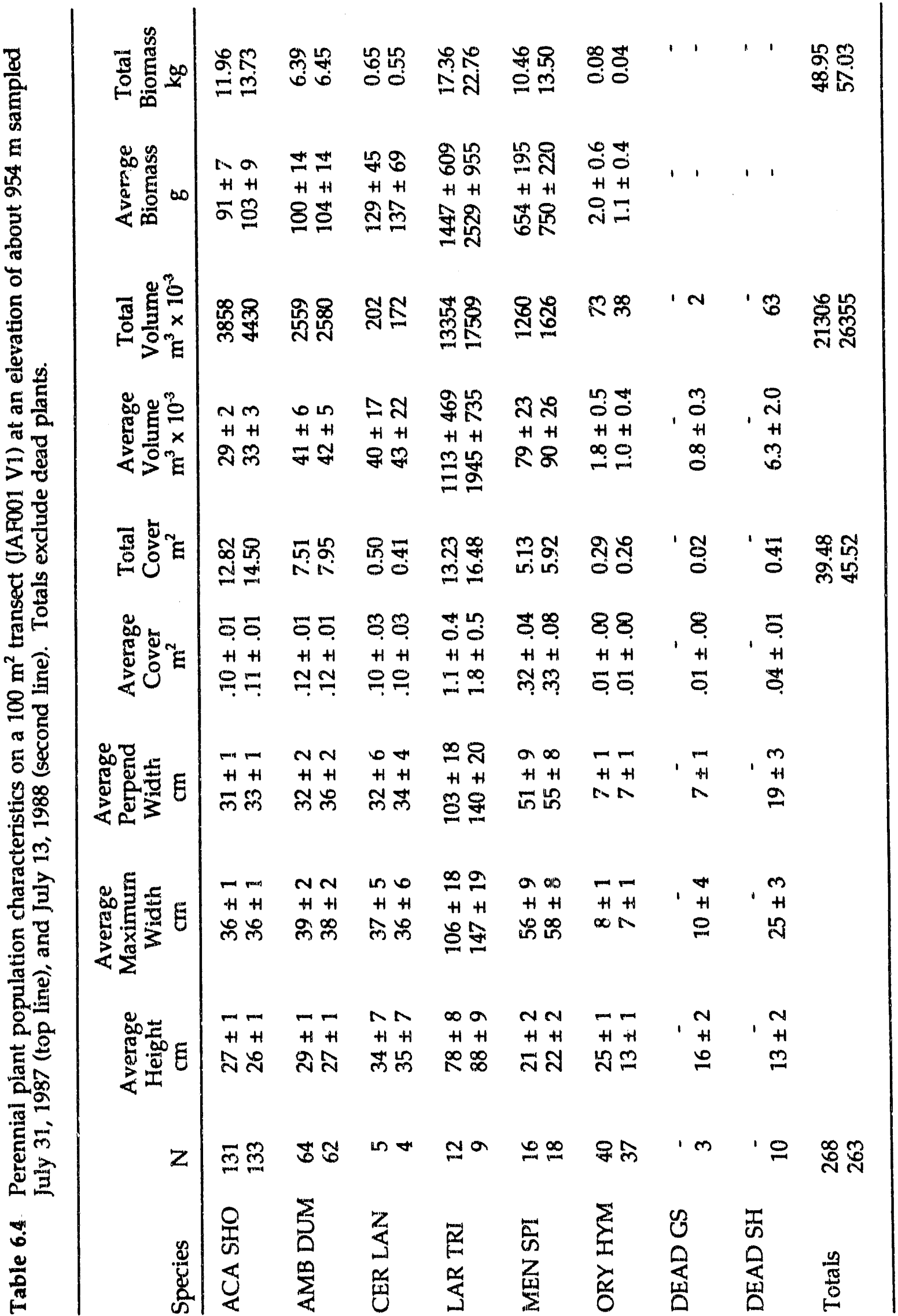




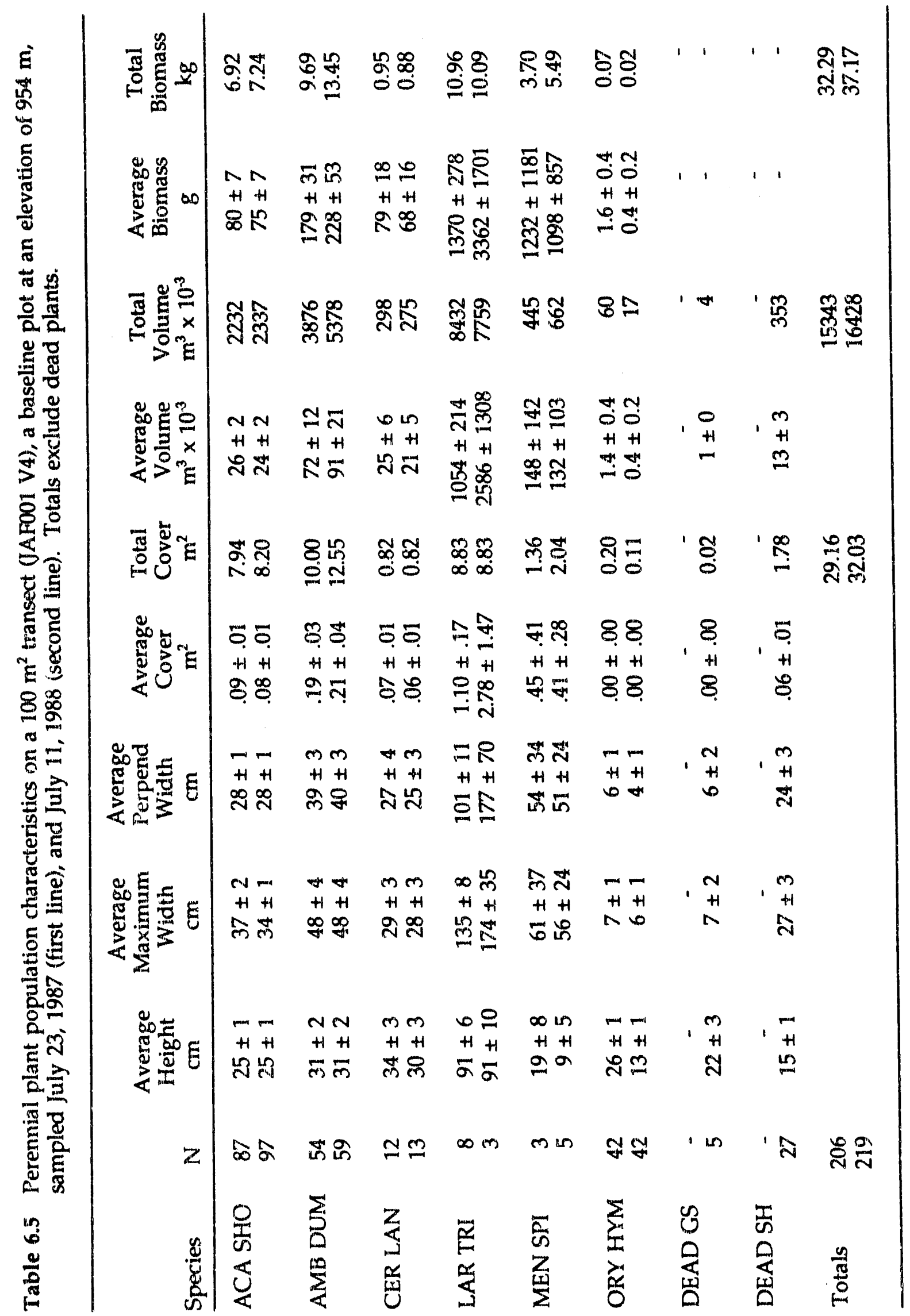


numbers and decreased average sizes. We concluded that at this site in Jackass Flats there was an approximate stasis in the plant population between 1987 and 1988.

The baseline plot in Frenchr an Flat (FRF001) is situated on a sandy, shallow-sloped bajada at 965 meters. It is near Beatle 's plot 23 (Beatley 1979), and the vegetation was dominated by Larrea tridentata. Plant populi tion characteristics were very similar in 1988 and 1987, except for the loss of some Hymenoclea salsola and Oryzopsis hymenoides plants (Table 6.6). In this case the losses were probably due to death of plants rather than failure to measure them. Hymenoclea salsola is a shrub which invades and grows well on disturbed areas and in washes on the NTS. It has had a young population in many areas on the NTS only since the early 1980s, following presumed germination events between 1978 and May 1983 (see Hunter et al. 1987). The largest $H$. salsola on plot FRF001 was $0.57 \mathrm{~m}^{3}$ in size, while the majority were generally small and young, the average being 0.034 in 1987 and $0.059 \mathrm{~m}^{3}$ in 1988 . Beatley (1979) reported average heights of $38 \mathrm{~cm}$ in 1963 and $45 \mathrm{~cm}$ in 1975 for the H. salsola on the set of plots which included plot 23, while our average heights were 23 and $27 \mathrm{~cm}$. The death of $H$. salsola plants on FRFOOl was concentrated in the smaller plants (15 of 17 that died were $<0.001 \mathrm{~m}^{3}$ in 1987), leading to an increase in average size. Similariy, Oryzopsis hymenoides is a herbaceous, short-lived species (West and Gunn 1974; Pearson 1975) which can be expected to fluctuate in numbers. The widths were improperly measured on FRF001 in 1988, and therefore data were not available to make a size comparison. On the whole, plot FRF001 exhibited an insignificant change in both numbers and plant sizes from 1987 to 1988, except for those two species.

The baseline plot in Yucca Flat (YUF001, 1237 meters) is on an alluvial fan with gravelly, loamy sand soils (Romney et al. 1973, sites 75 and 76). It is in a vegetative community called "Grayia-Lycium" by Beatley (1976). The dominant species were Ephedra nevadensis, Lycium andersonii, and Grayia spinosa in 1987-88, although the most numerous perennial plant species was Ceratoides lanata (Tables 6.7 and 6.8). In 1988 most species declined somewhat in average volume, especially Hymenoclea salsola and the bunchgrasses (Oryzopsis hymenoides, Sitanion jubatum, and Stipa speciosa). This is similar to the situation in Frenchman Flat, except that there was little decline in numbers of $\mathrm{H}$. salsola. Changes in numbers of Lycium andersonii and Grayia spinosa should not be considered significant, as they look dead when dormant, and some 1987 workers considered some dormant plants dead and, therefore, did not measure them. The decline in numbers of Sphueralcea ambigua (a herbaceous perennial) on transect V2 (Table 6.7) should be considered real, the result of death among the smaller individuals. Again, there was an approximate stasis among the dominant shrubs, but a decline in size and/or numbers in the herbaceous and short-lived species.

One other plot had data from two years (Table 6.9). A 200- $\mathrm{m}^{2}(2 \times 100 \mathrm{~m}$ ) plot in Area 3 (YUF012, 1239 meters), used as a control transect for another study in 1986, was remeasured in 1988. It was in a very mixed shrub population dominated by Tetradymia glabrata, Atriplex canescens, Ceratoides lanata, and Ephedra nevadensis, growing on a sandy soil. The numbers of 


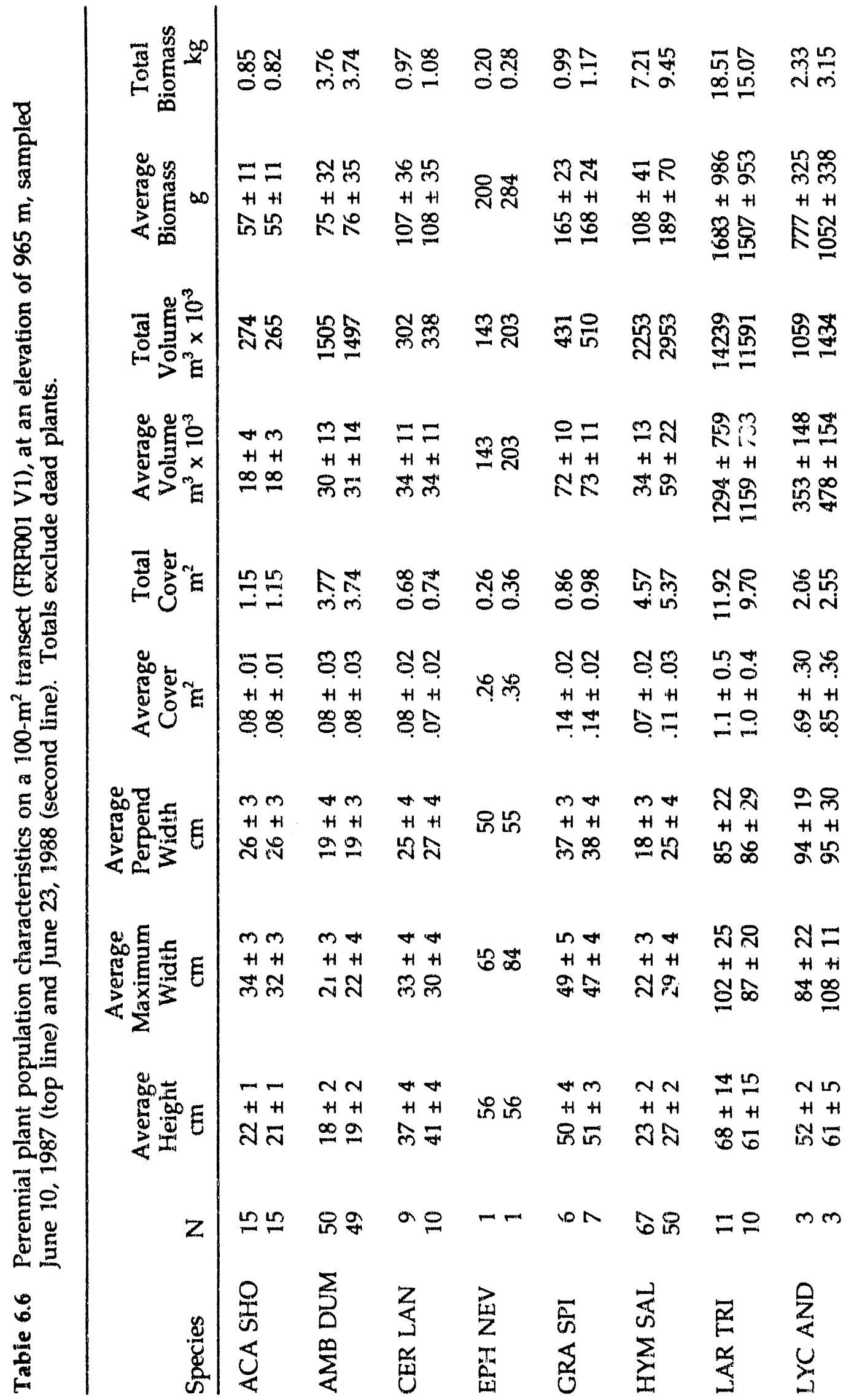




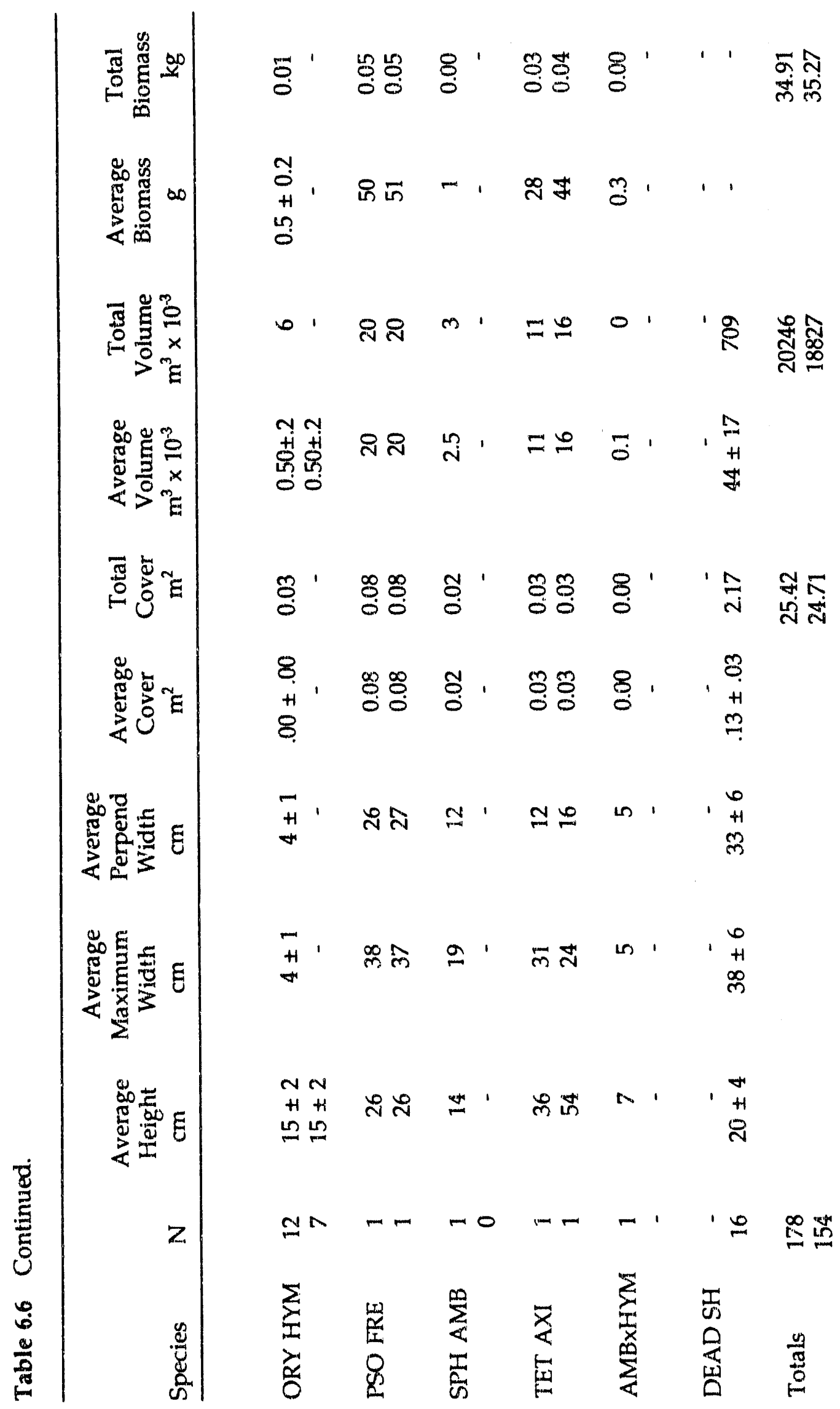

$\frac{1}{1}$ 


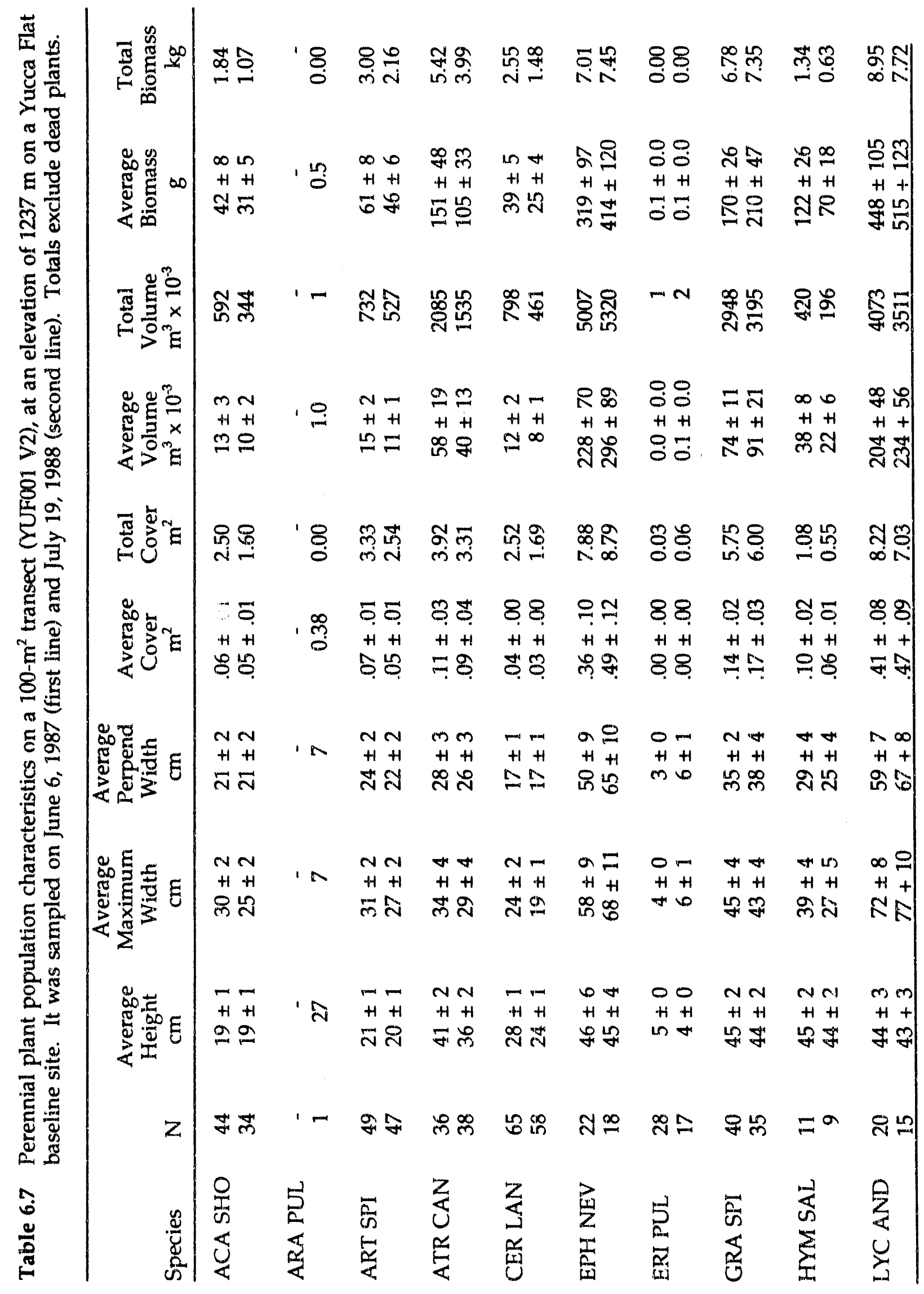




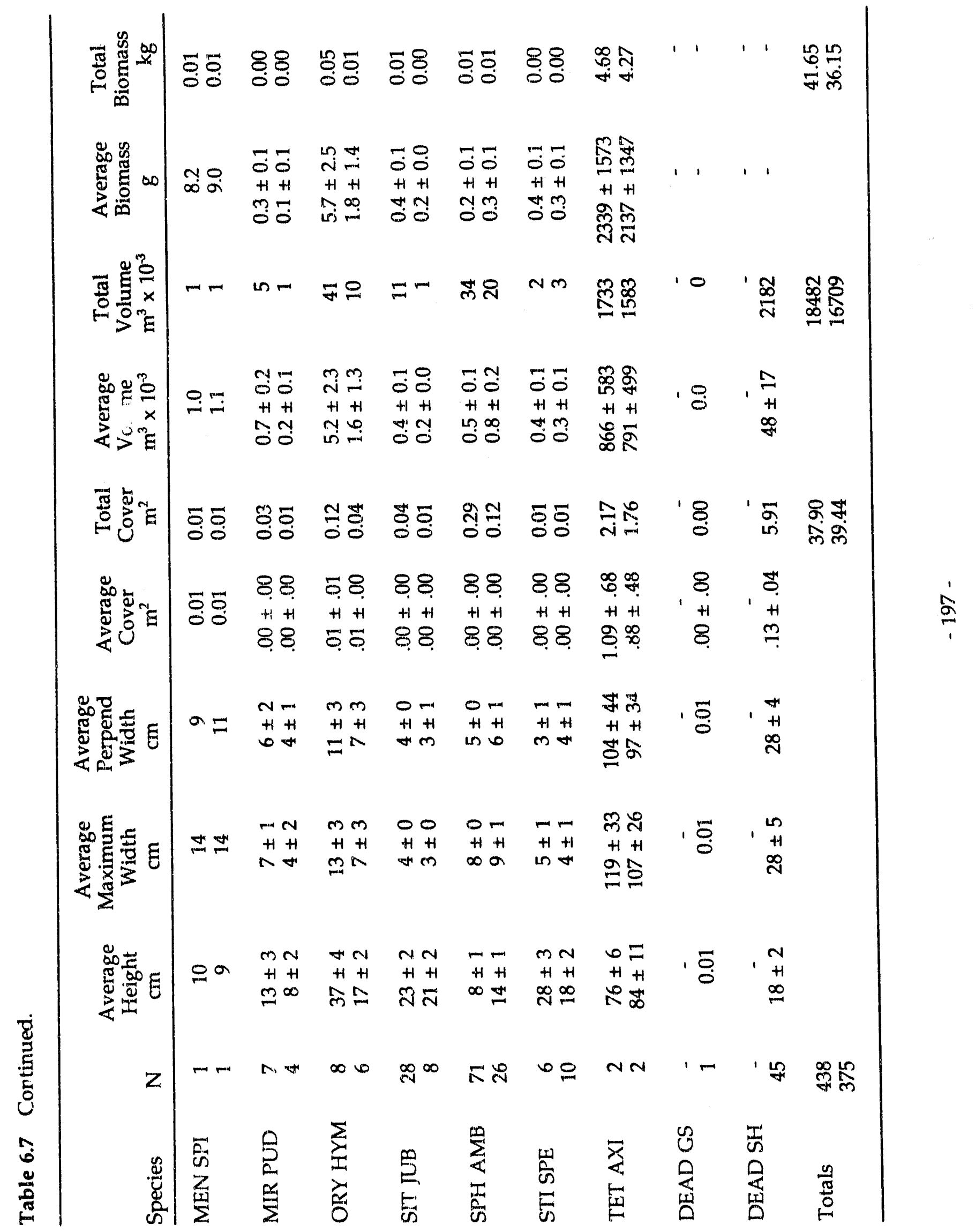




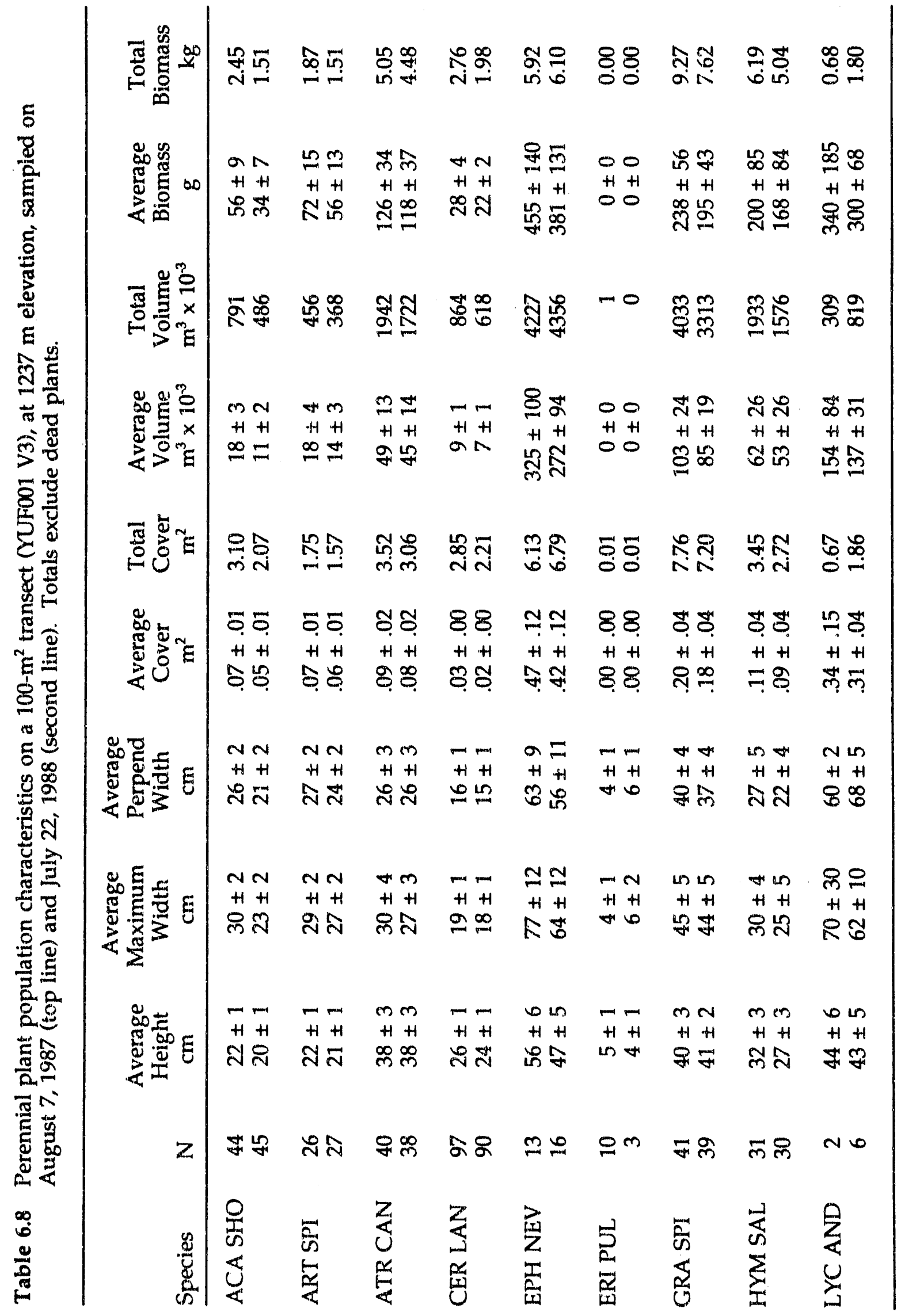




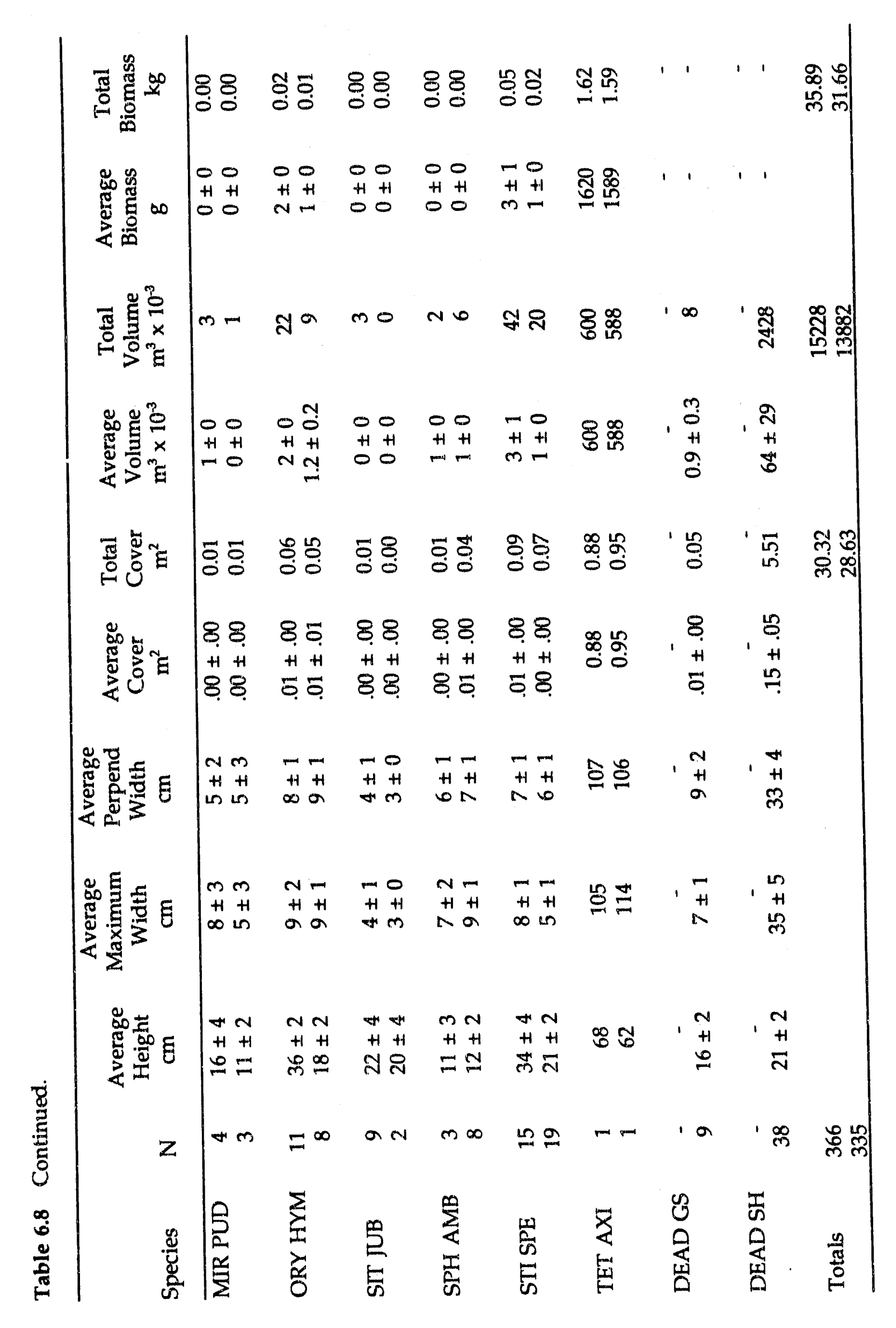




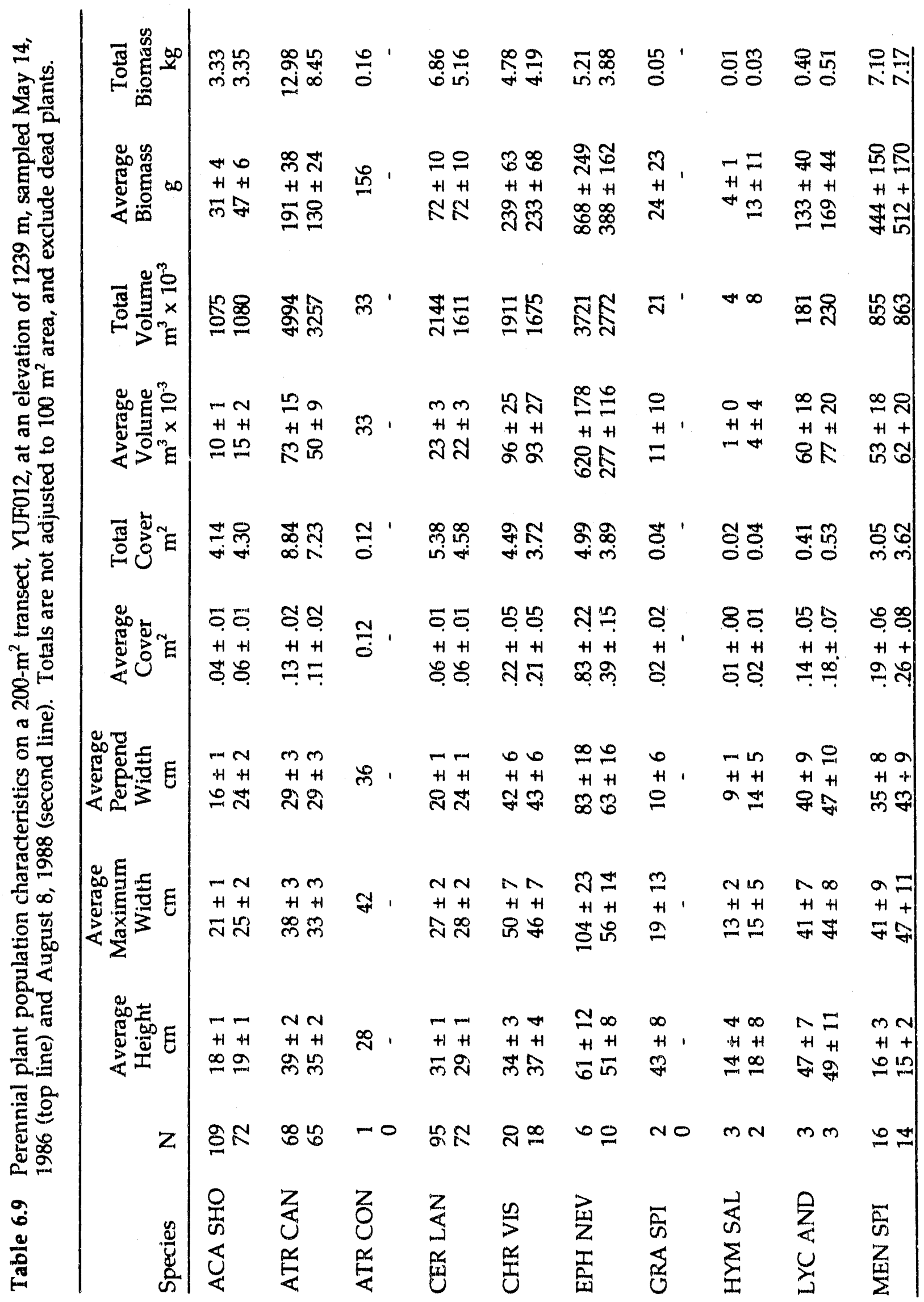




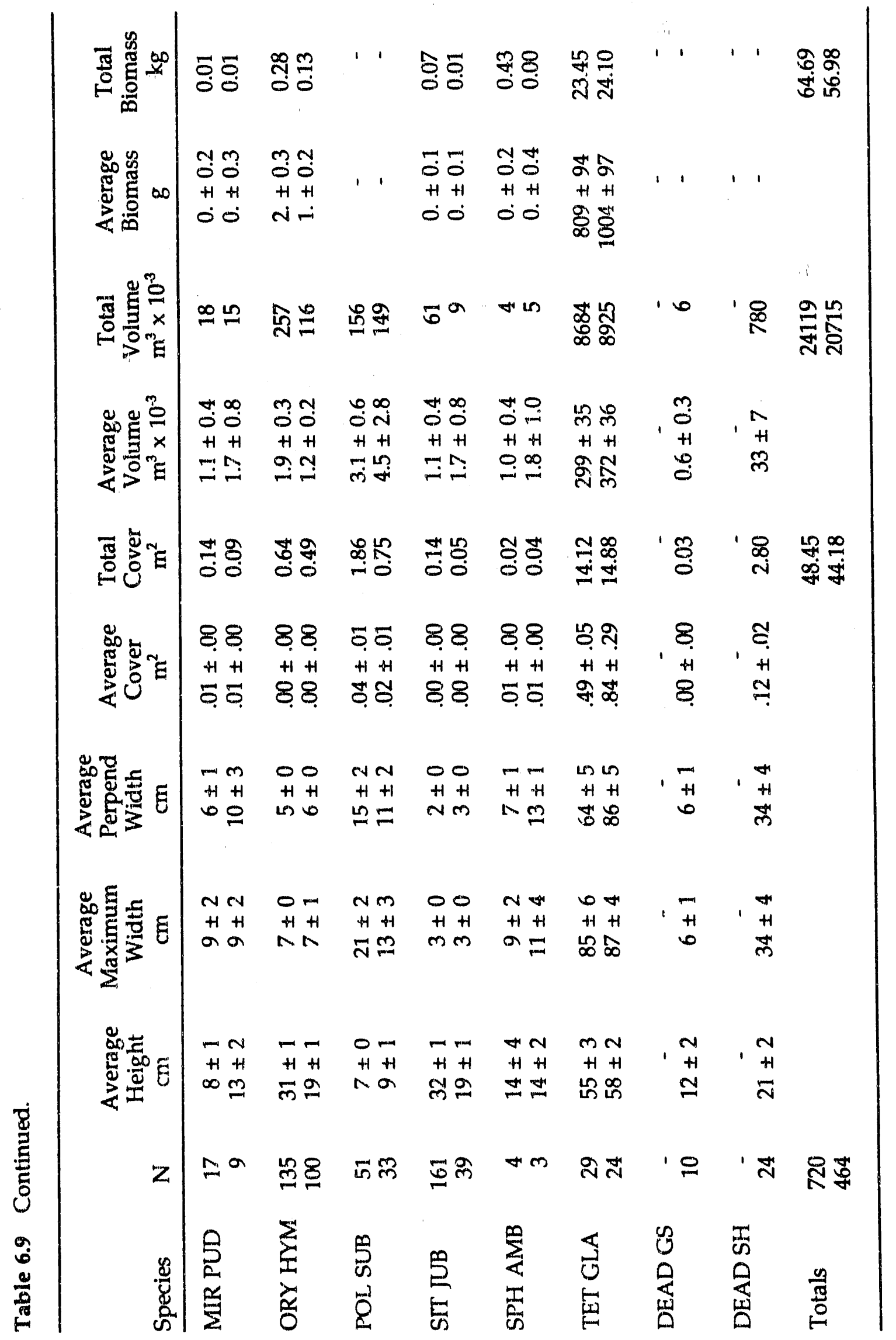


plants countsd declined from 720 to 464 between 1986 and 1988, with the major declines again occurring in the herbaceous species (the bunchgrasses Oryzopsis hymenoides, Sitanion jubatum, Stipa speciosa, and Sphaeralcea ambigua, Mirabilis pudica, and Polygala subspinosa). One bunchgrass species, Situnion jubratum, declined precipitously from 161 to 39 plants.

Another way of looking at these plo's was to consider only the matchable plants and estimate growth and dieback of those individuals. This is somewhat different from looking at population means. It was statistically more powerful to look at individual data, but the sample populations differed because some plants were not matchable. The several reasons for failure to match could possibly create some bias in results. First, dead plants were not matched with live predecessors because the species determination is often ambiguous on dead plants, and the live size of zero for dead plants gives a growth coefficient of negative infinity. Therefore, the matched population may have been selected for healthier, surviving individuals rather than average plants. Second, small plants were harder to see, and often hidden under larger shrubs, so they may have been less frequently matched, again selecting larger, presumably healthier plants for matching. Third, plants with several individuals in a small area could not be readily distinguishet from one another with the collected data, and were therefore excluded from the matched populations. Finally, plants well separated from others were easier to match, selecting individuals in what may be more open, favorable microhabitats. In spite of those factors, however, the median 1987 volume of matched plants was the same as the median volume of the whole 1987 population of plants with a probability of $>90 \%$ on plot JAFO01 ( $t$-test as proposed by lglewicz in Hoaglin et al. 1988), indicating biases due to matching difficulties were more potential than real. (The percentages of shrubs matched, by plot, are included in Table 6.10.)

With those factors in mind, the measurements for matcked plants were used to calculate average growth rates. In many cases, growth coefficients (defined as [in(V2/V1)]/time in years, where $V=$ live volume) can be significant indicators of the vigor of a plant population. The logarithmic growth coefficients resulted from model of plant growth which was considered more realistic than linear models, as in most plants absolute growth is proportional to initial size. The model used here is appropriate for those plants which have rot attained their full potential size (Erickson 1976). This is appropriate in desert situations, where water, rather than genetics, generally limits size, and plants shrink and grow with changes in rainfall. A rough idea of the meaning of the growth coefficients can be obtained from the calculated times required for a plant to double in size (Table 6.11). Shrinkage rates (negative $k$ ) are at analogous halving times.

The results of analyses of the two Jackass Flats transects (Table 6.10) show that, overall, there was no change in size from 1987 to 1988. It appeared that between 1987 and 1988 one species (Oryzopsis hymenoides) decreased in size, while the other five were static (growth not significantly different from 0 ). These results are consistent with the population data (Tables 


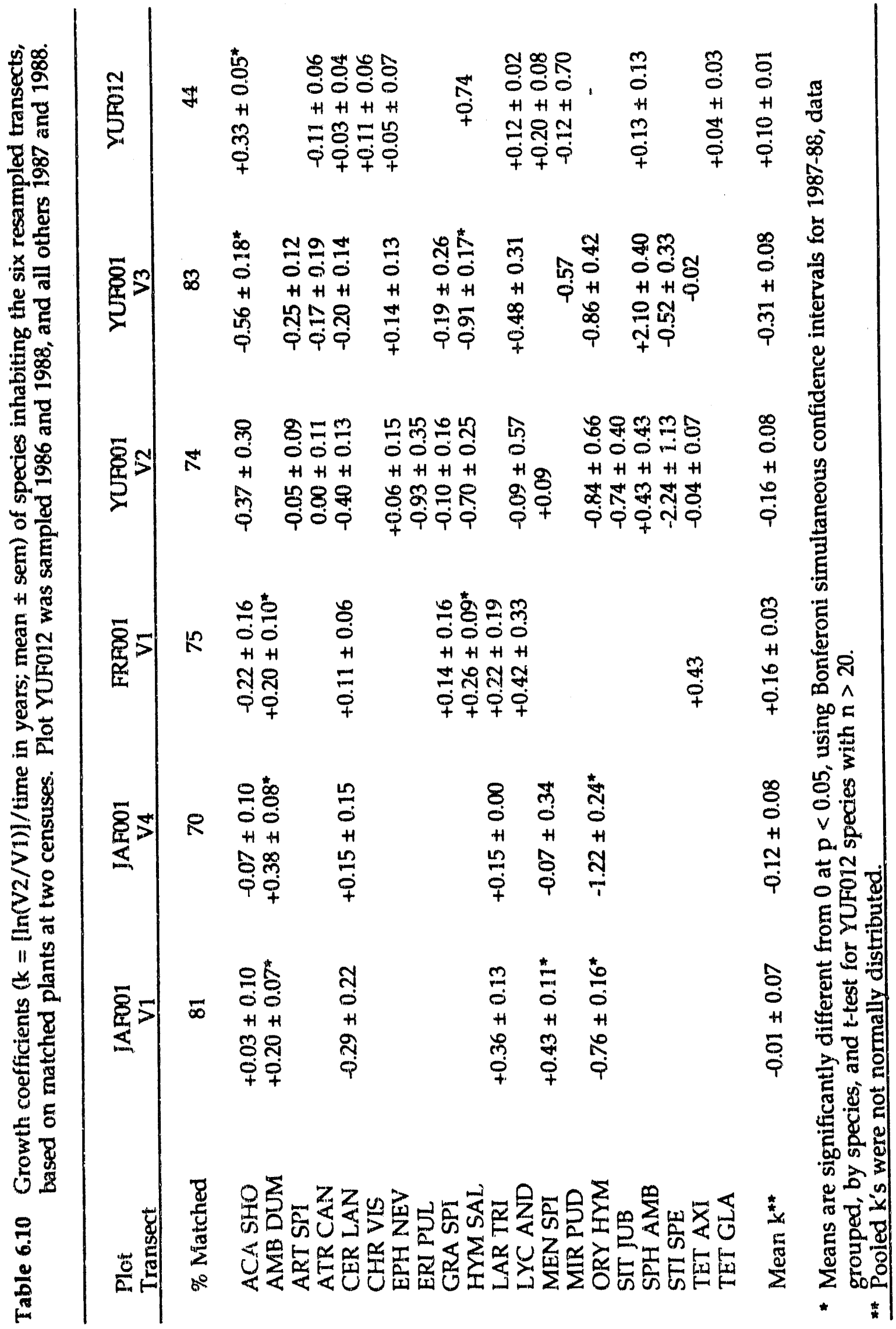

尽 
Table 6.11 Times to double in size associated with different growth coefficients $(k=[\ln (V 2 / V 1)] /$ time in years).

\begin{tabular}{cc}
\hline$k$ & time,years \\
\hline 0.1 & 6.93 years \\
0.2 & 3.46 years \\
0.4 & 1.73 years \\
0.7 & 361 days \\
1.0 & 253 days \\
2.0 & 126 days \\
3.0 & 84 days \\
4.0 & 63 days \\
5.0 & 51 days \\
7.0 & 36 days \\
9.0 & 28 days \\
\hline
\end{tabular}

6.4 and 6.5 ), but the ability to use statistical tests on the numerous individuals allowed a better interpretation of changes in size. It should be noted that to show that plants neither grew nor shrank over a one-year period may be a significant observation, rather than an indication of measurement error.

For comparison, a population of Ambrosia dumosa seedlings growing on a disturbed area in Jackass Flats grew at rates up to $k=8.6$ for a thirteen month period (1983-4; Hunter 1989).

Values of growth coefficients from the other plots with matched plants were similar (Table 6.10). They showed a general stasis in plant sizes from 1987 to 1988 , with only a few species either growing or shrinking significantly. The median growth coefficient for 1986 to 1988 (YUF012, Table 6.10) showed a positive but small growth $(k=+0.06, n=203)$, which was significantly different at $p<0.01$ by $t$-test on the difference in medians (Iglewicz, see Hoaglin et al. 1983) from the 1987-88 growth coefficient on the nearest plot YUF001 (median $k=-0.20, n=513$ ). These data suggest plants in Yucca Flat grew slightly from 1986 to 1987, then shrank in 1987-1988.

\section{MEASUREMENTS AT NEW LOCATIONS}

Results of censuses on new plots do not show the changes occurrirg, but provide a one-time picture of vegetative conditions of sites during summer of 1988. They are presented here in order of altitude. 
A roadside in Frenchman Flat (5-03 Road; 977 meters) and an adjacent control area were censused (Tables 6.12 and 6.13). The road has been abandoned and barricaded for approximately 20 years and the shoulders have not been maintained. The area measured was the two meters adjacent to the asphalt covering. Perennial shrubs growing on the verge were long-lived dominant species. They were generally small and young. There were no dead plants. The control area had 9 dead plants and 61 live ones. The distribution of dead categories (see Methods) was significantly different at $p<0.001$ (Chi-square), as $70 \%$ of the roadside plants had no dead parts (category 0 ), compared to only $18 \%$ of those on the control. Seventy-six percent of control plants were 1-25\% dead (category 1). Unusually healthy plant populations near the roadbed are a common phenomenon in the desert, probably resultirig from several factors, including reduced competition (note the lower cover and total live volume values), runoff water received at the road edge, and storage of water under the asphalt.

An area in Mercury Valley denuded of perennial shrubs (MER002, 1076 meters) by gophers and other small animals (Hunter et al. 1980) had a very reduced perennial plant cover (Table 6.14). The only shrub inhabiting the area was Stanleya pinnata a species which is not grazed significantly, apparently because of bad flavor (Hunter 1987). It sometimes occurs on seleniferous soils (Munz 1974) and is therefore sometimes toxic to wildlife from selenium accumulation. A few other herbaceous perennials made up the sparse population. Dead shrubs were largely S. pinnata. The two Sitanion jubatum were unusual for Mercury Valley, but did not constitute a significant population. In contrast, the control area (MER003; 525m to the NE, 3618 feet) had total cover of about $44 \%$ (Table 6.16) by a mixed shrub community dominated by Larrea tridentata. There were no Stanleya pinnata on the control transect. S. pinnata is normally restricted to areas where the dominant, drought-tolerant vegetation is absent, probably due to an inability to compete for water with the dominant shrub species.

An area scraped for cleanup of radioactive waste (Waste Management Consolidation Site $3 b$ ) in Yucca Flat (XUF011, 4075 feet) had no live perennial plants. It is about $100 \mathrm{M}$ north of the control area sampled in both 1986 and 1988 (YUF012, Table 6.9).

Two disturbed sites sampled were in large areas whose vegetation was removed by blasts from above-ground nuclear bomb testing during the 1950s (T1 and T3). The perennial plant population on YUF013 (T3) consisted of two species, Mirabilis pudica, a herbaceous perennial which dies to ground level yearly, and the bunchgrass Oryzopsis hymenoides. Cover was even lower than on the gopher-denuded areas, but did not consist of grazing-resistant species. As with other blast areas in Yucca Flat, the annual weed Salsola paulsenii was abundant (see ephemeral report). The control area for this site was YUF012 (Table 6.9), about $225 \mathrm{~m}$ east of YUF013. 


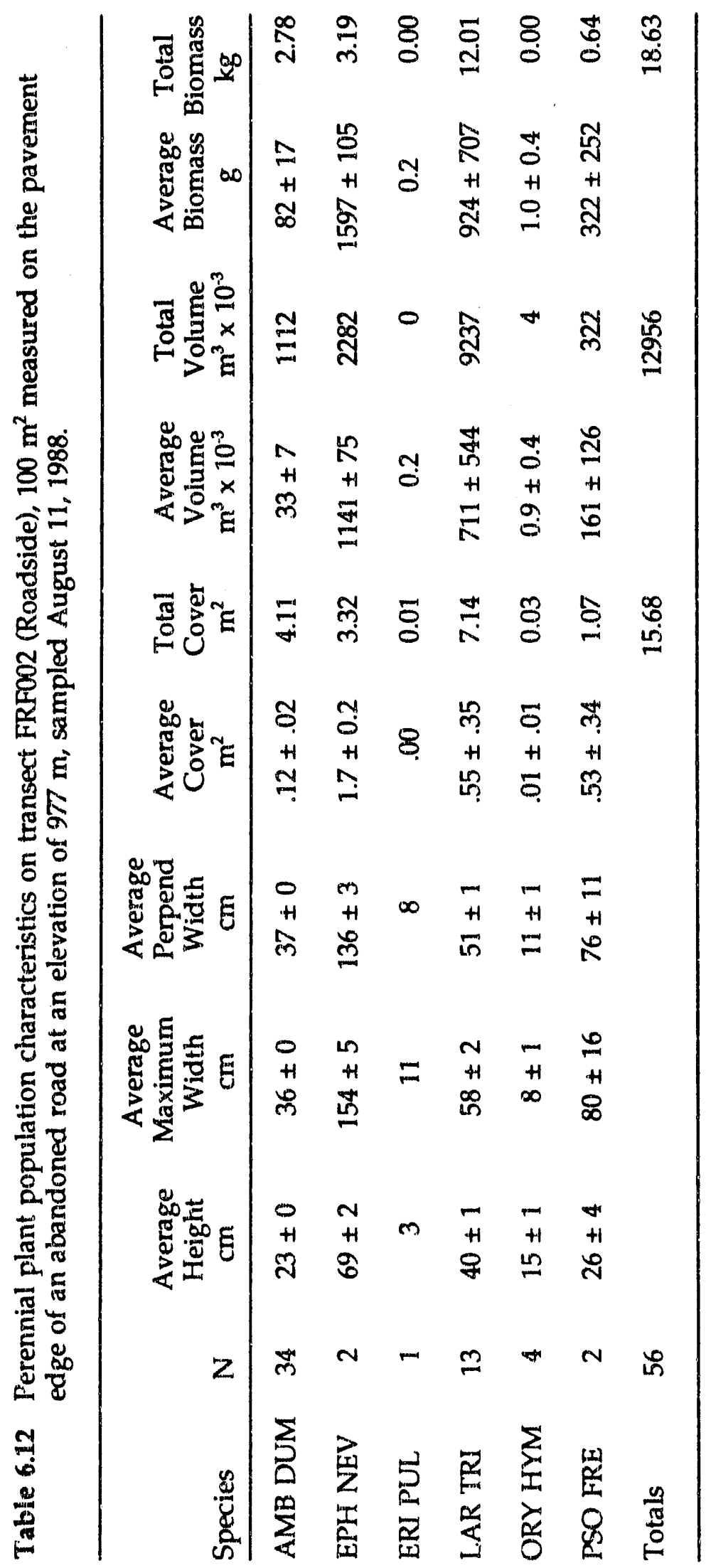




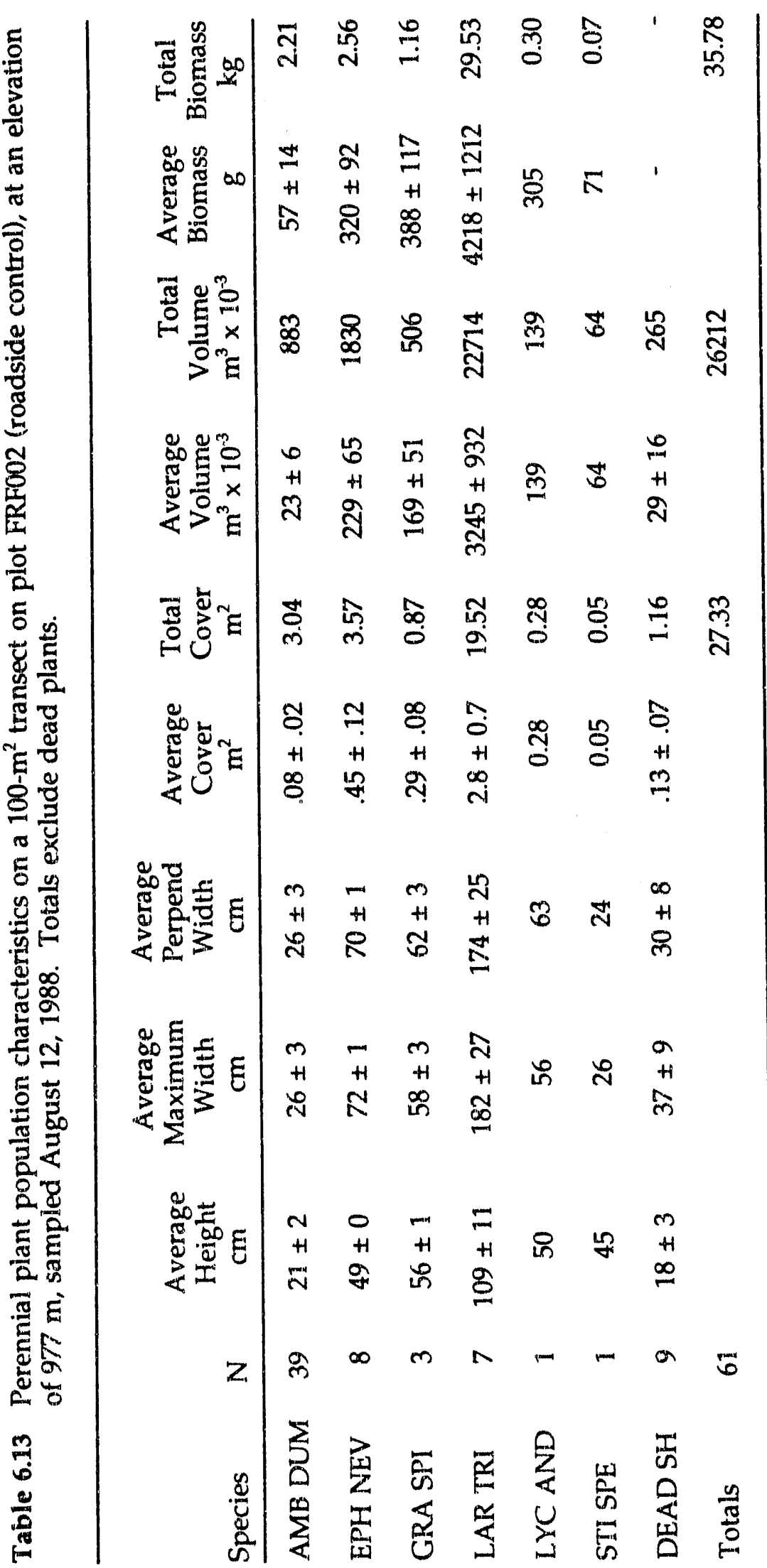




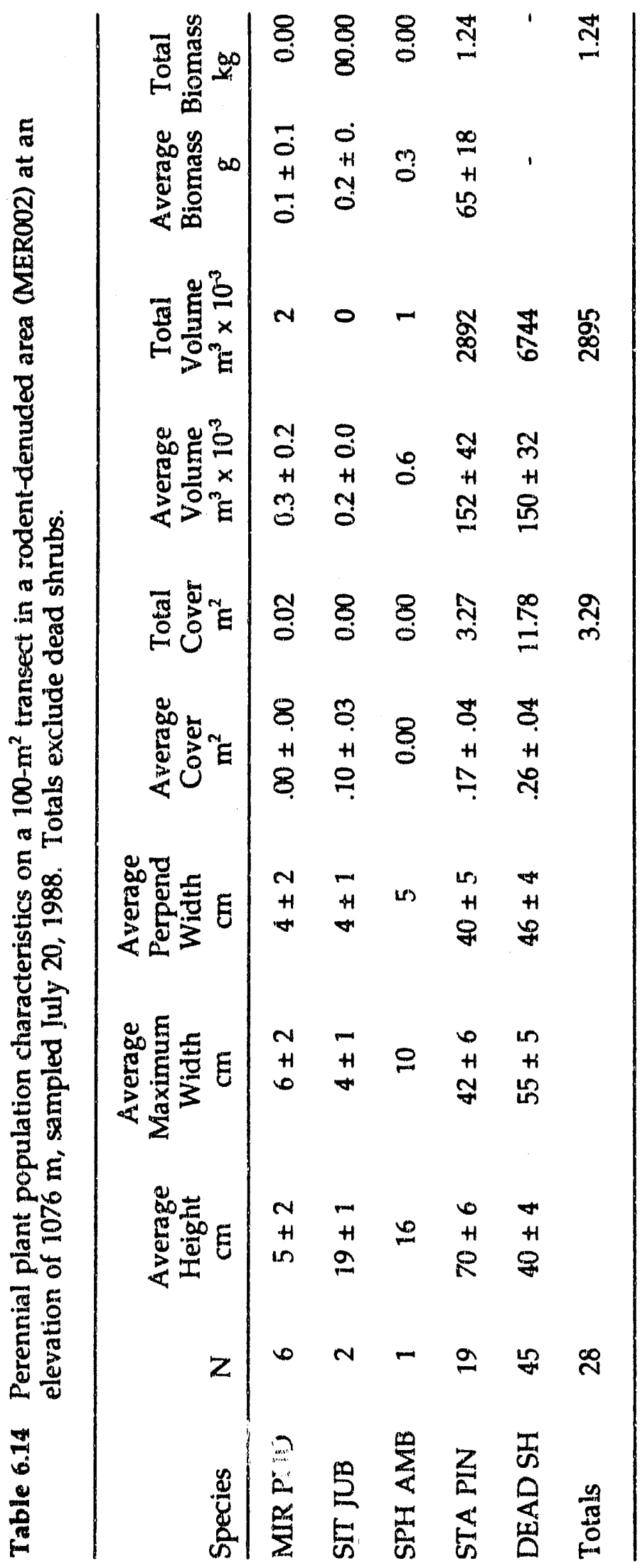

$\stackrel{\infty}{\circ}$ 


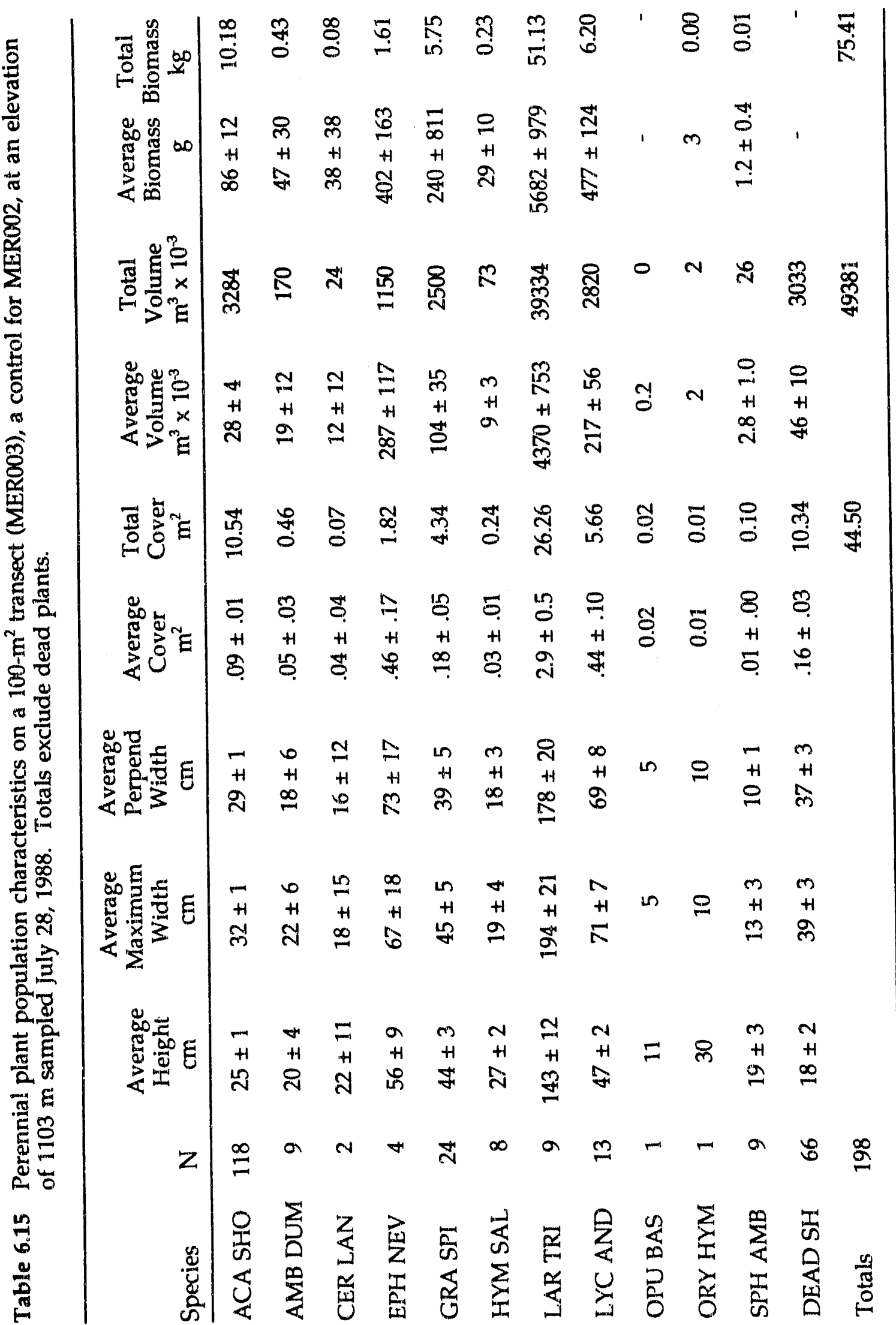




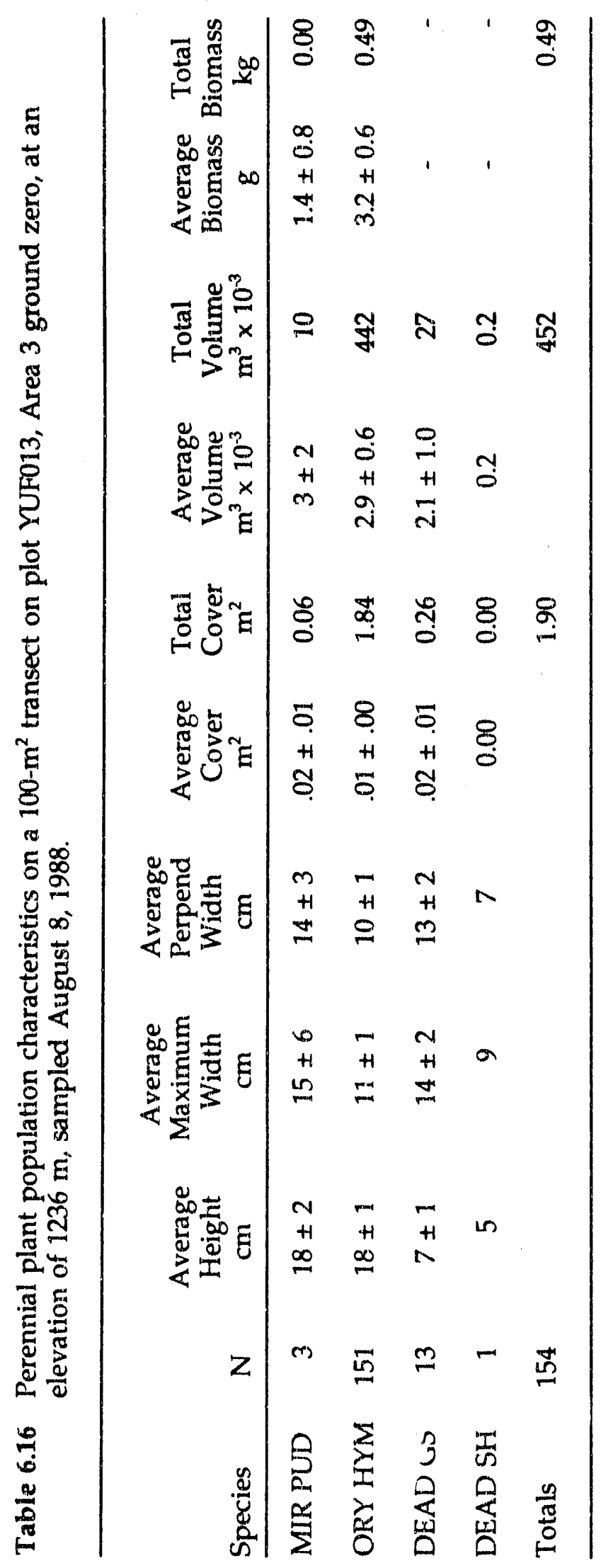

$\stackrel{1}{N}$ 


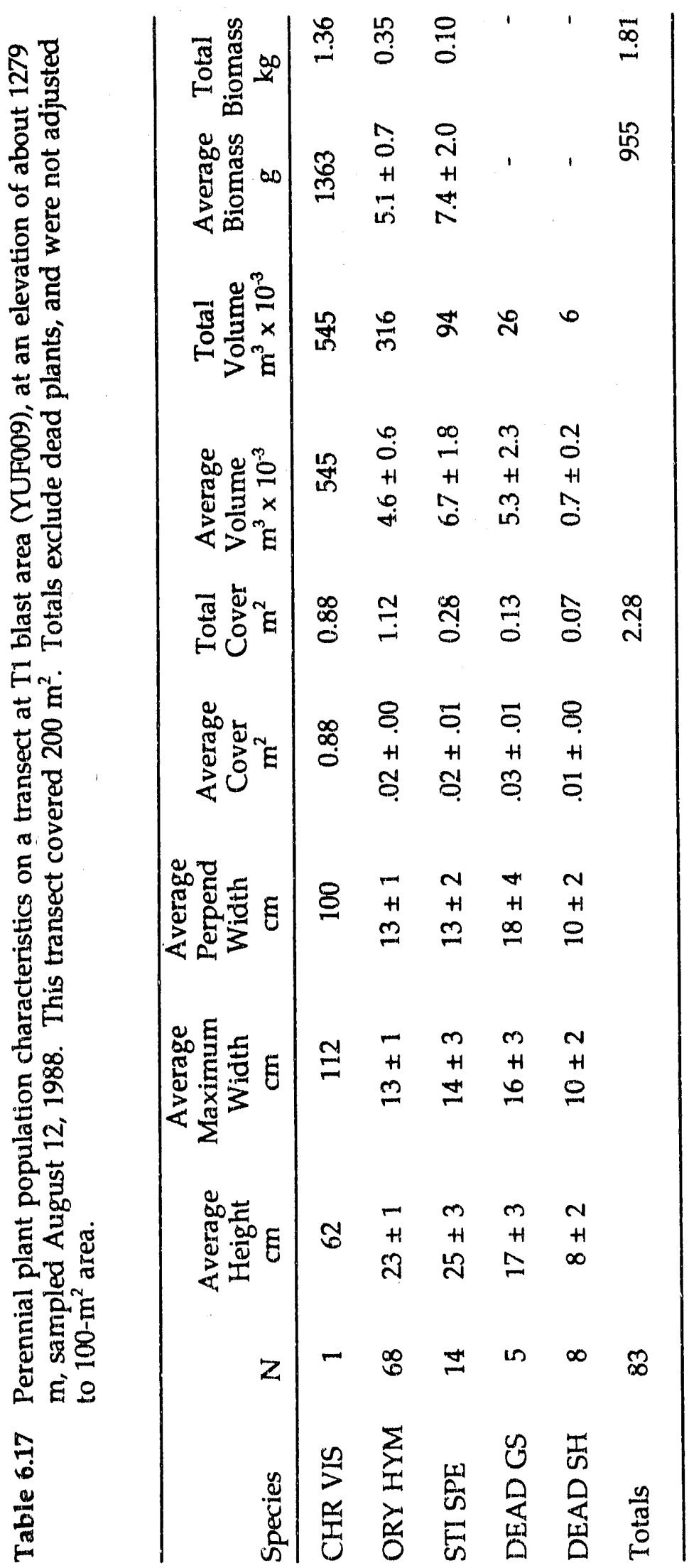




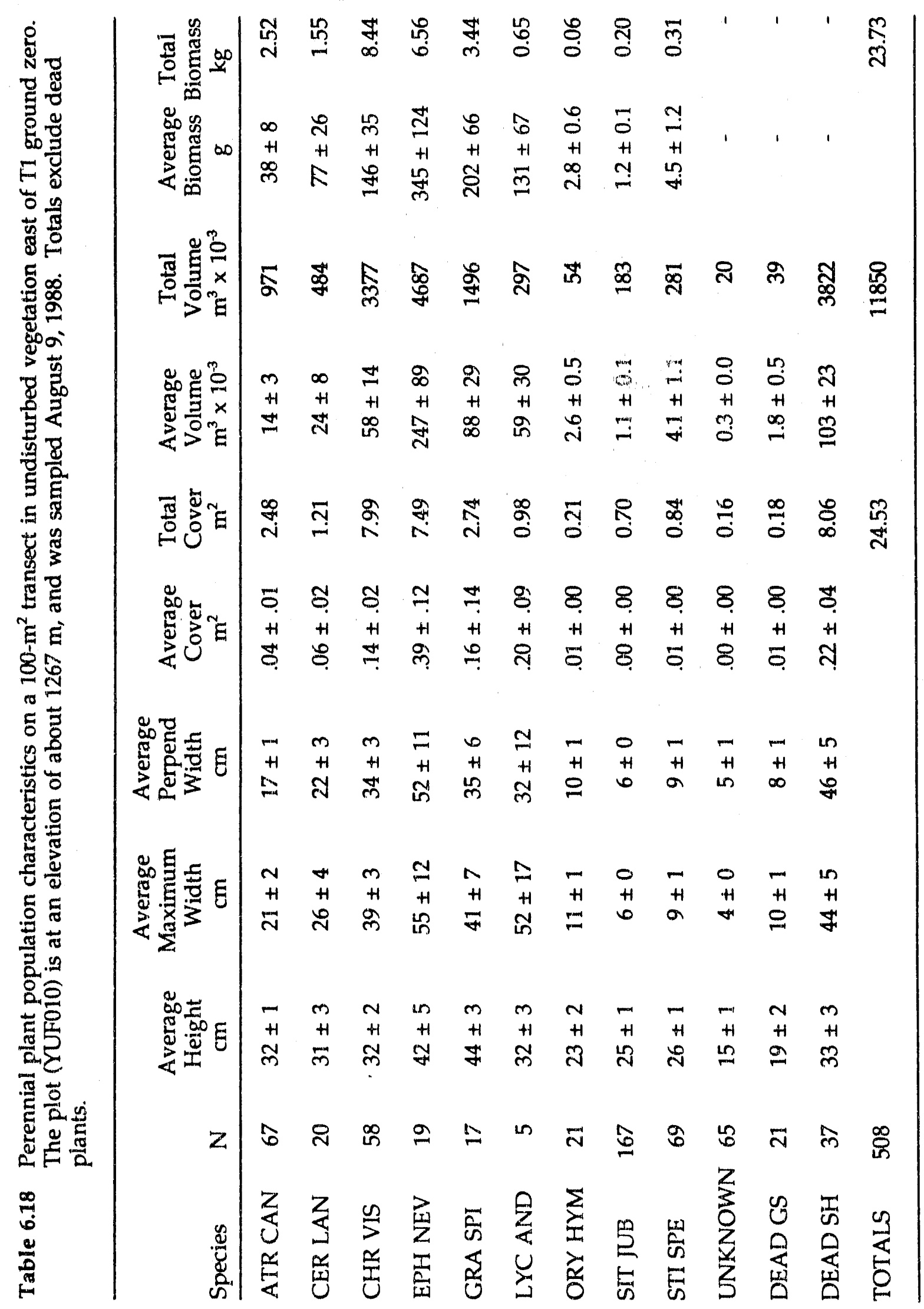


The blast area at $\mathrm{T} 1$ sampled in 1988 (Table 6.17; YUF009, 1279 meters) was similarly dominated by Oryzopsis hymenoides, with the addition of some Stipa speciosa, another bunchgrass. One Hymenoclea salsola was present in $200 \mathrm{~m}^{2}$. The dead shrubs were old dead stumps which could not be identified. They were possibly remains of shrubs present before the blasts occurred from 1952 to 1957 . Its control area, around $600 \mathrm{~m}$ east, was a wellmixed shrub community dominated by Ephedra nevadensis and Chrysothamnus viscidiflorus (Table 6.18; YUF010, $1267 \mathrm{~m}$ ).

Dr. E. M. Romney monitored grass seedlings at two sites within the blast area at T1 from 1959 through 1987. The seedlings were marked after germination events with small wooden stakes, and those plants were followed for two years after marking (E. M. Romney, personal communication). New seedlings were apparent in 1971, 1978, and 1983; Table 6.19 shows the results of those studies. It should be noted that the numbers are not densities of grass on a site, but numbers of seedlings only; established plants were not followed. These data illustrate the nature of the influx of grasses to the blast area, i.e., germination in response to particular rainfall patterns, followed by erratic establishment depending on the weather.

In addition to the grass seedling data, in 1986 and 1987 Romney and Hunter measured plant cover in five plots established by Romney and Rhoads in 1959, when there were no perennial plants. Table 6.21 summarizes the results of those measurements. They demonstrate the presence of the bunchgrasses and Hymenoclea salsola as in the BECAMP plots measured in 1988 (YUF013 and YUF009).

Another blast area sampled was that around the peaceful nuclear excavation test which produced Sedan crater. The sites sampled were described earlier by UCLA (Martin 1963), Allred et al. (1963), and by Hunter et al. (1987). As at the other blast areas, the area near the crater (YUF016, $305 \mathrm{~m}$ from GZ) was populated solely by the bunchgrasses Oryzopsis hymenoides and Stipa speciosa (Table 6.21) and ephemerals (see ephemerals report). From May 1983 to July 1988 the numbers of bunchgrasses had increased from $0.19 / \mathrm{m}^{2}$ (Hunter et al.1987) to $1.08 / \mathrm{m}^{2}$. Cover was slight, less than $1 \%$. At $914.4 \mathrm{~m}$ from GZ, which was past the area of deposits from the crater (Martin 1963), a similar area (YUF017) was also populated by grasses, but the shrub Hymenoclea salsola dominated the vegetation, contributing 

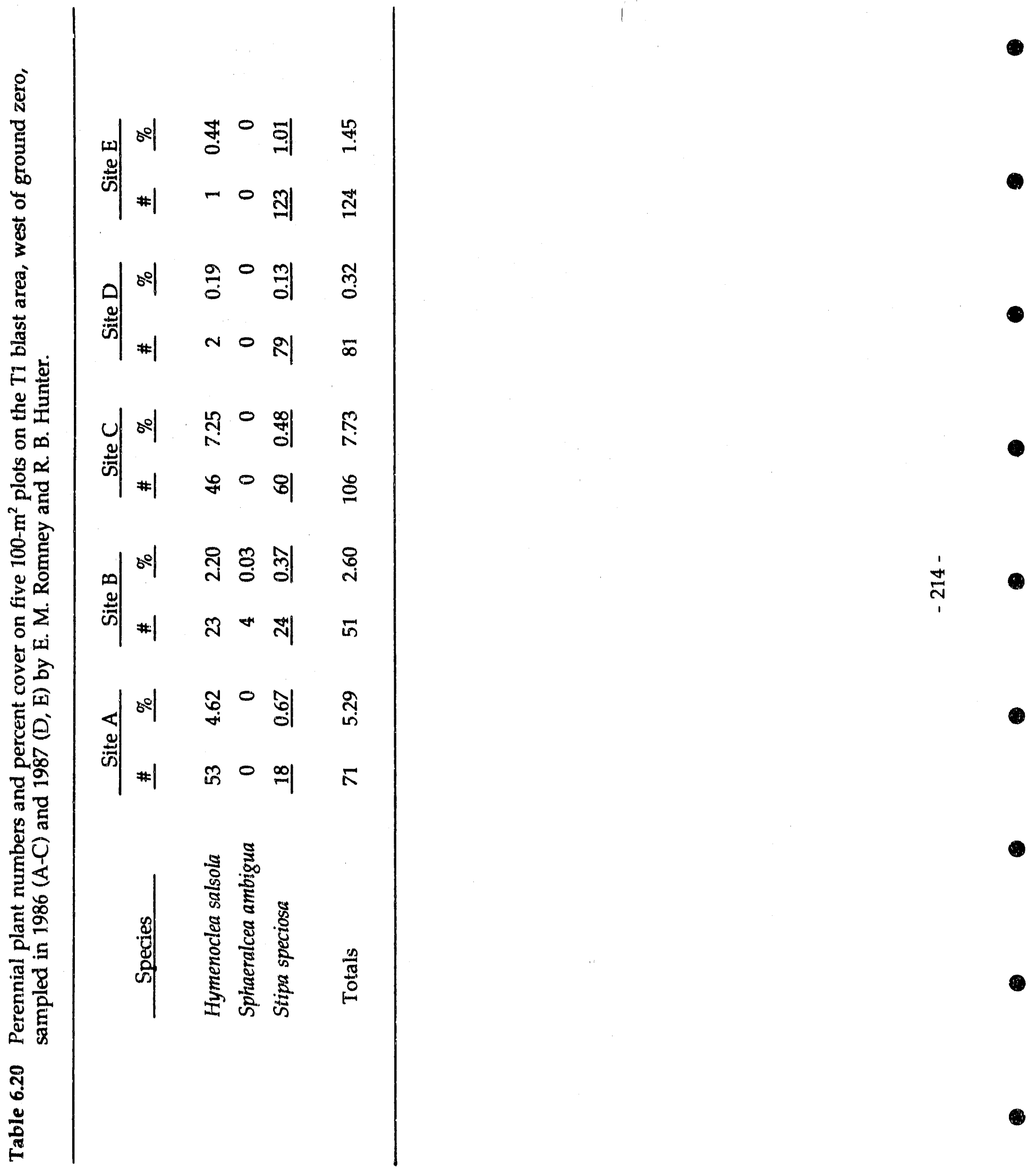


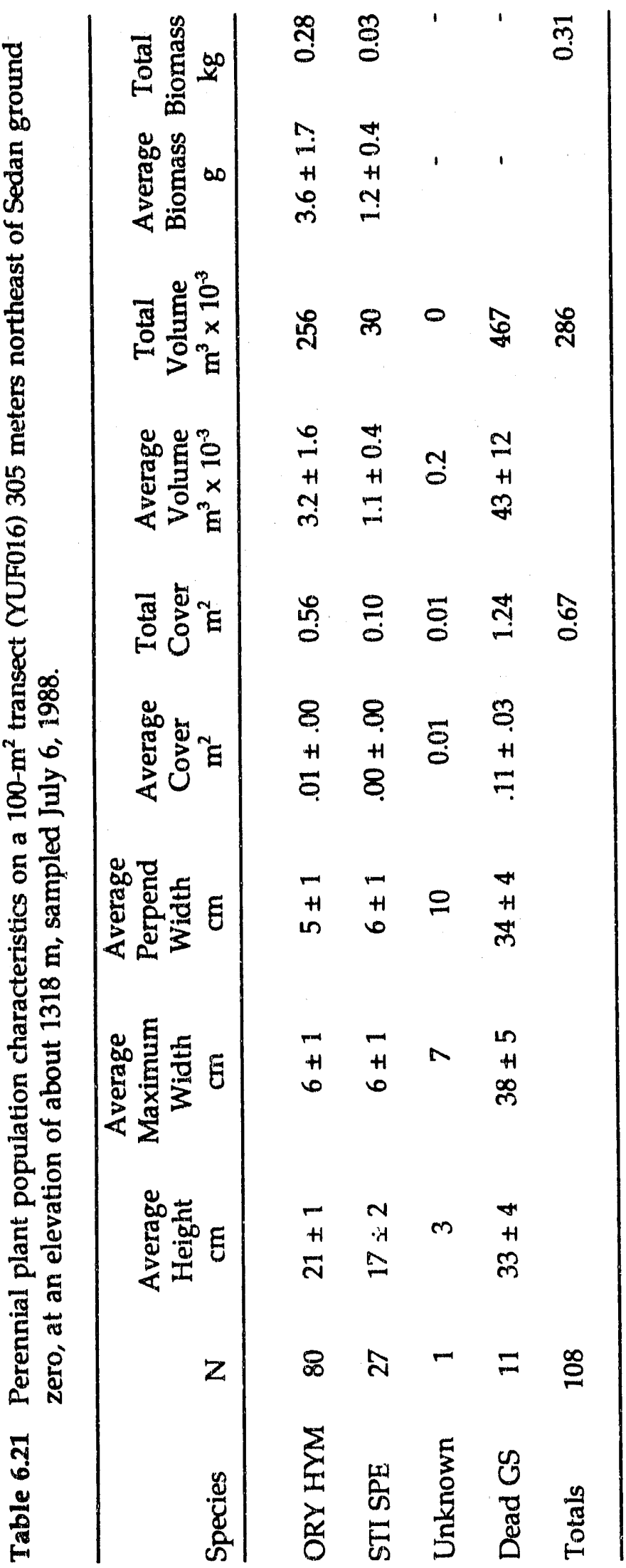


almost $8 \%$ cover (Tabie 6.23 ). H. salsola increased from $3 / 100 \mathrm{~m}^{2}$ in 1983 to $58 / 100 \mathrm{~m}^{2}$ in 1988, while the other shrub present, Lycium andersonii, declined from three to two individuals (there was one in 1976, none in 1964; Hunter et al. 1987). At $1524 \mathrm{~m}$ from GZ, just past the edge of the area of blast removal of shrubs (Martin 1963), the vegetation was dominated by Coleogyne ramosissima (YUF018, Table 6.23), whose population remained intact after the blast. Total shrub numbers declined from $99 / 100 \mathrm{~m}^{2}$ in 1963 (Martin 1963) to $82 / 100 \mathrm{~m}^{2}$ in 1988 (Table 6.23, excluding grasses). Data from 1962-1964 (H. O. Hill, unpublished data) showed cover at this approximate location to be dominated by Coleogyne ramosissima(14\%) and Grayia spinosa (7\%), but the G. spinosa was absent in 1988. In 1975 one G. spinosa was seen in a 29- $\mathrm{m}^{2}$ area sampled (Hunter, R. B. and H. O. Hill, unpublished data). This location was not sampled in 1983.

These data on the vegetation in the blas rea of the Sedan event show a succession of the Salsola-dominated vegetation to perennial bunchgrasses during the 1970 s and invasion by the shrub $H$. salsola to the edge of the throw-out zone (i.e., to $762 \mathrm{~m}$ from GZ) during the $1980 \mathrm{~s}$.

Two new baseline sites were sampled in 1988, one on Pahute Mesa and one on Rainier Mesa, to make more cornplete the number of vegetative communities sampled. The plot on Pahute Mesa (PAM001, 6310 feet) was in an Arternisia nova community with a few small scattered Juniperus osteosperma (junipers) and a rare Artemisia tridentata. The almost total dominance of cover by $A$. nova is seen in Table 6.24. Ephedra nevadensis and the bunchgrasses Orysopsis hymenoides and Sitanion jubatum contributed significantly to the numbers of plants. The plot is on a very rocky soil on a knoll. The Ephedra nevadensis plants were small in comparison to lower-altitude sites, and they appeared to have reproduced mostly vegetatively, with rhizomes. It was not possible to determine which of the small Ephedra shoots were individual plants and which parts of a clone, so the numbers are dependent on an arbitrary classification.

The baseline plot on Rainier Mesa (RAM001, 2283 meters) was chosen where lizard and small mammal studies were performed by Brigham Young University in the 1960s (Tanner and Hopkin 1972; Jorgensen and Hayward 1965). The community was very dense and diverse (Table 6.25), and only $54 \mathrm{~m}^{2}$ were measured due to lack of time. The dominant species were Pinus monopinylla (pinyon pine) and Cowania mexicana. Numerical dominance was by the grass $P$ on sandbergii, the small shrub Linanthus nuttallii, and Artemisia tridentata (big sage). Scattered Juniperus osteosperma occurred in the area, but all were missed in the small transect sampled. A summary of the vegetative characteristics measured in 1988 is presented in Table 6.26, which shows live cover, volume, and biomass estimates for the sites sampled. Excluding the Rainier Mesa plot, there was no significant correlation of altitude with cover, volume, or biomass. Cover and altitude on undisturbed plots correlated significantly $(r=+0.70 ; p<0.01)$ only when Rainier Mesa was included. 


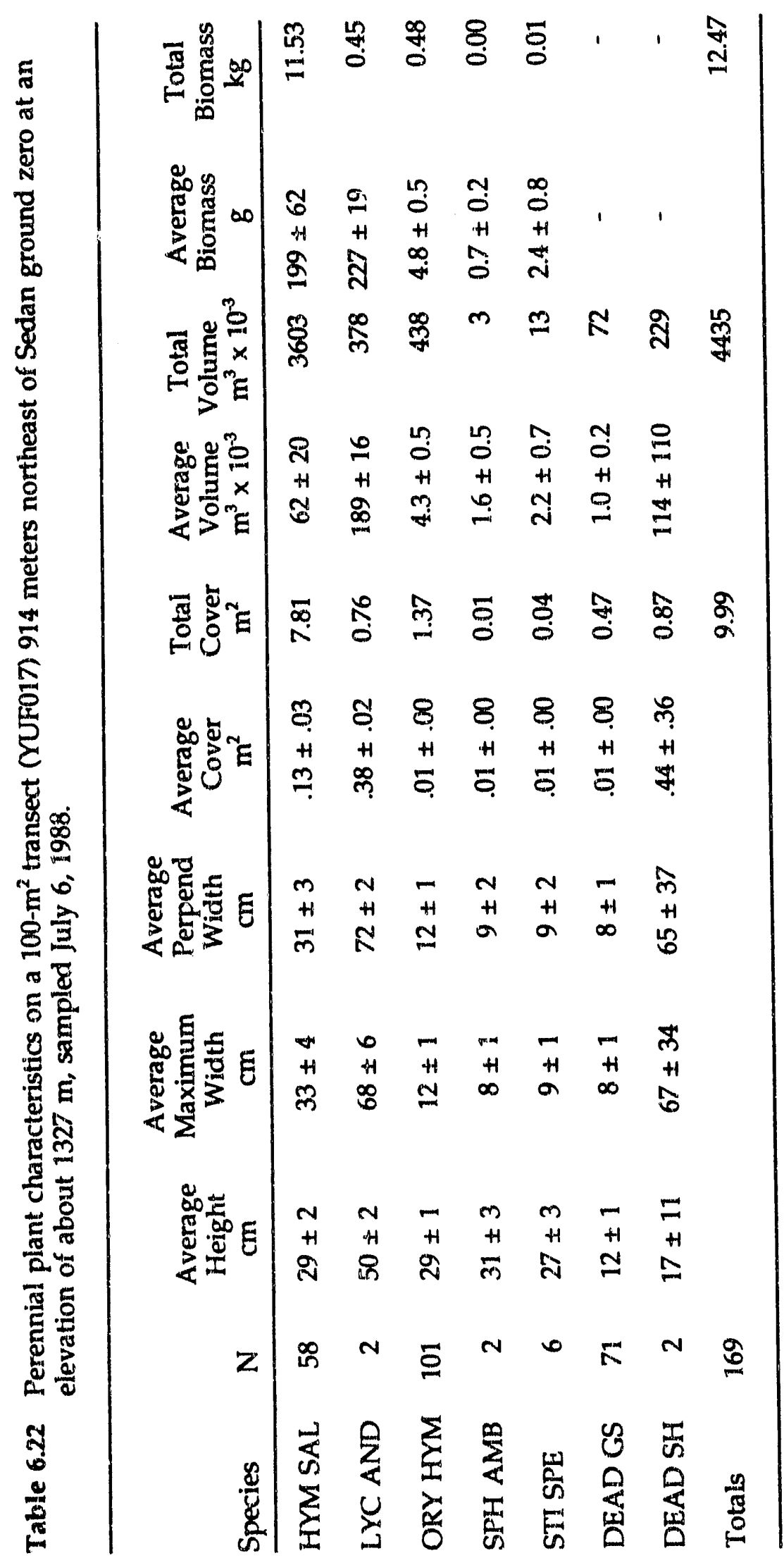




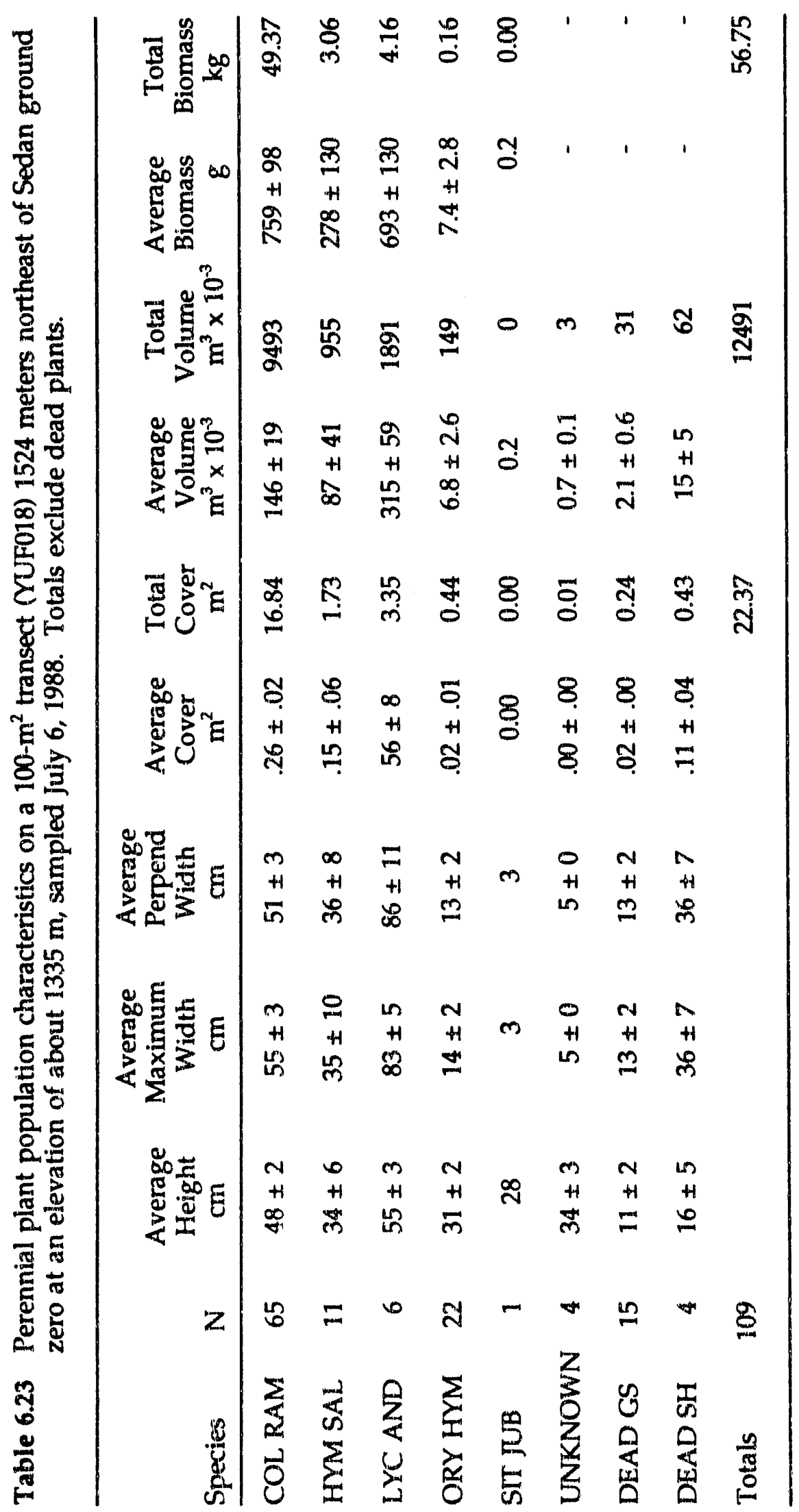

$\frac{1}{\sqrt{2}}$ 


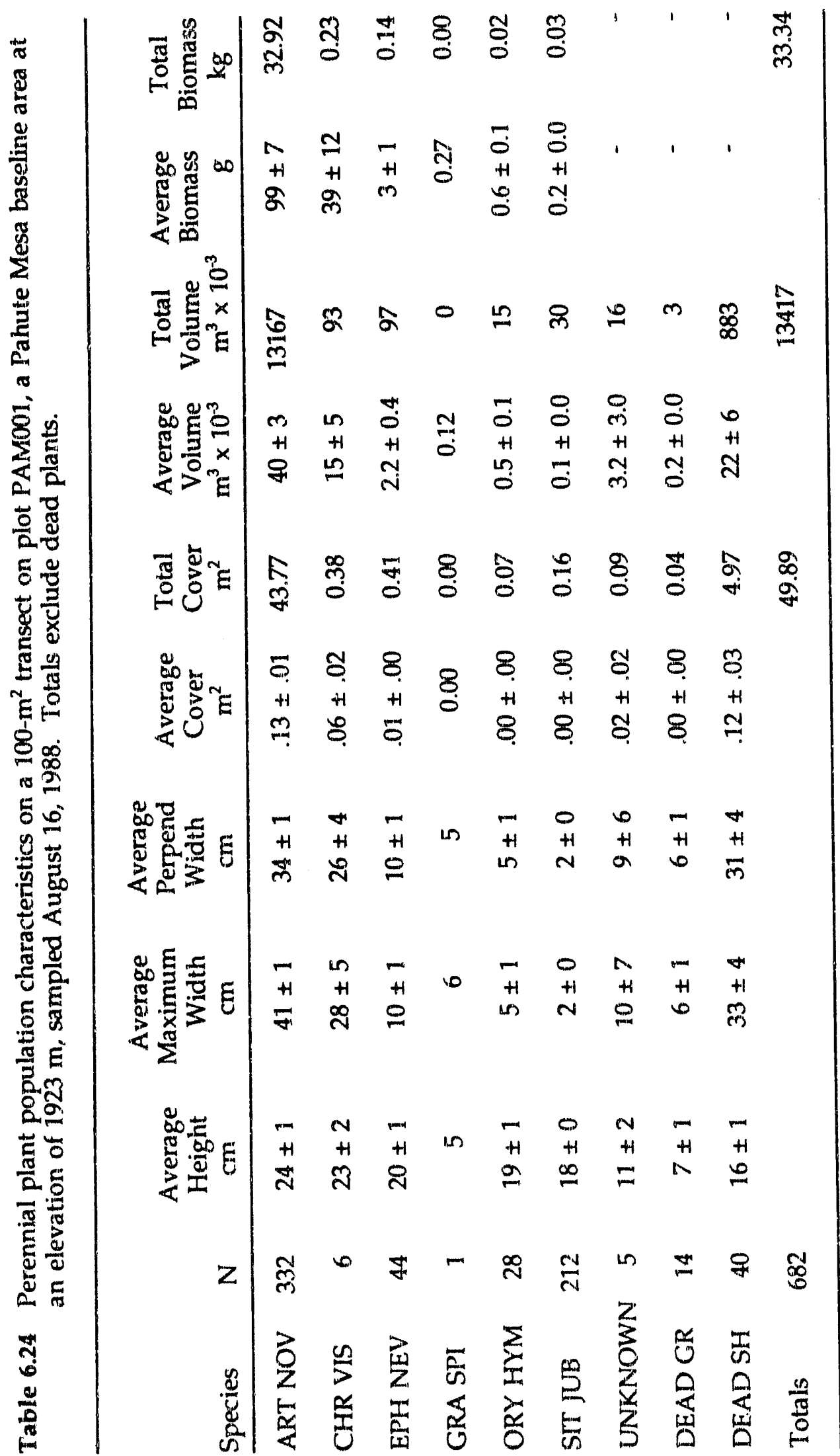




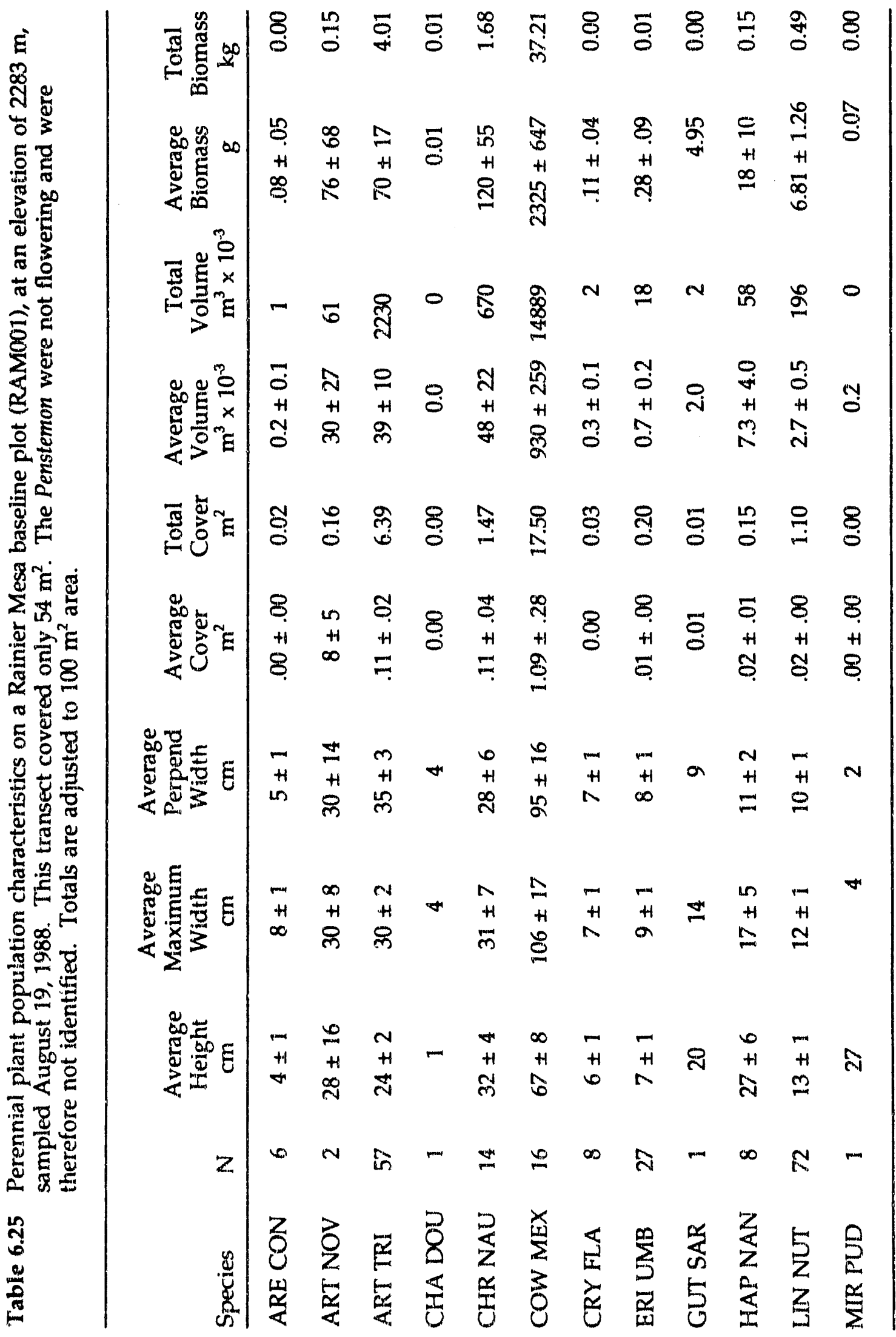




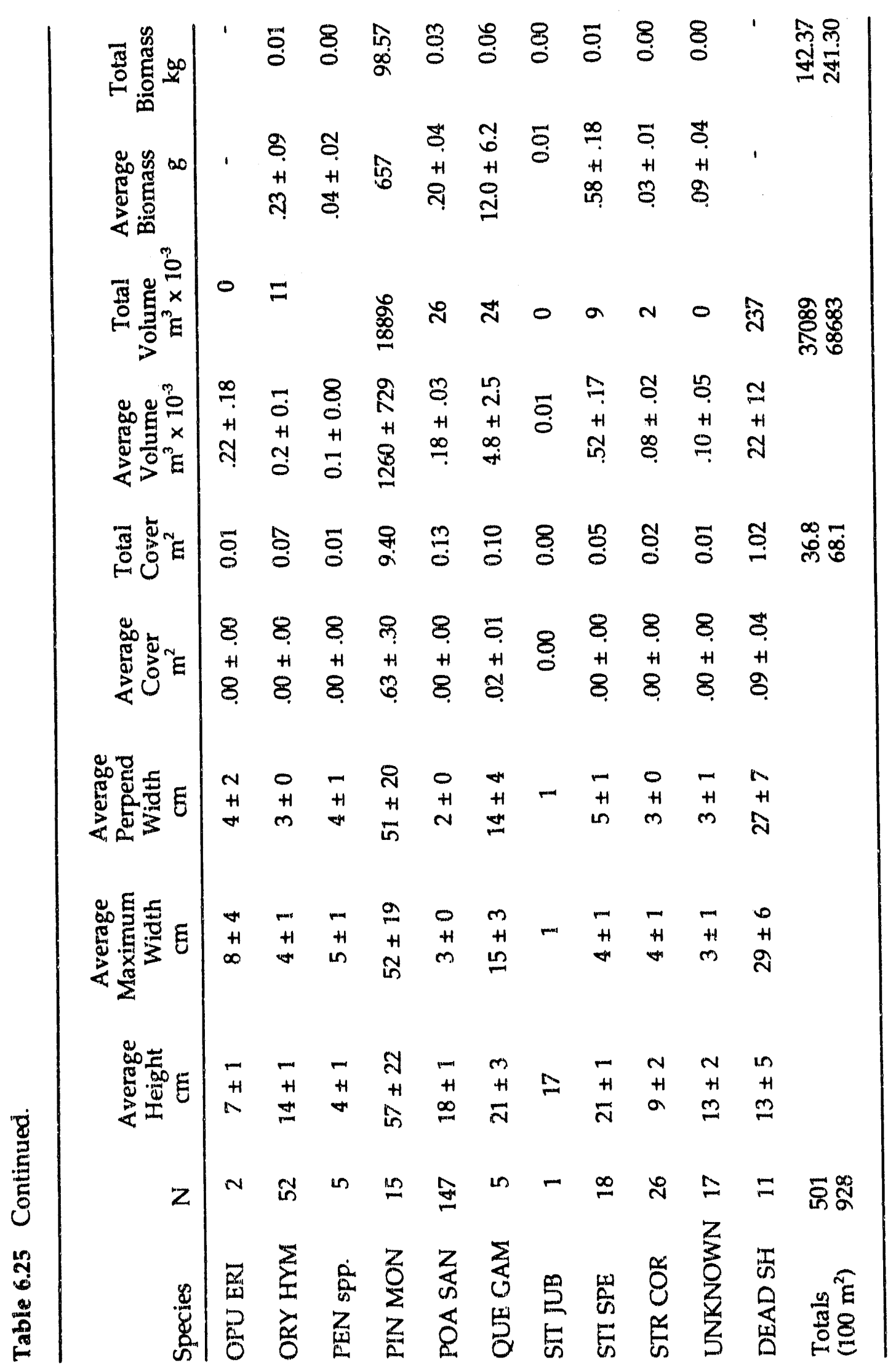


Table 6.26 Numbers of live plants, live cover (\%), total live volume $\left(\mathrm{m}^{3} / 100 \mathrm{~m}^{2}\right)$, and estimated biomass $\left(\mathrm{kg} / 100 \mathrm{~m}^{2}\right)$ and altitudes (meters) for sites sampled in 1988.

\begin{tabular}{|c|c|c|c|c|c|c|}
\hline \multicolumn{2}{|c|}{ Site } & \multirow{2}{*}{$\begin{array}{c}\text { Altitude } \\
954\end{array}$} & \multirow{2}{*}{$\frac{\mathrm{n} / 100 \mathrm{~m}^{2}}{263}$} & \multirow{2}{*}{$\begin{array}{c}\text { Cover } \\
46\end{array}$} & \multirow{2}{*}{$\begin{array}{c}\text { Volume } \\
26\end{array}$} & \multirow{2}{*}{$\begin{array}{c}\text { Biomass } \\
57\end{array}$} \\
\hline JAF001 & V1 & & & & & \\
\hline & V4 & 954 & 219 & 32 & 16 & 37 \\
\hline FRF001 & V1 & 965 & 154 & 25 & 19 & 35 \\
\hline \multirow[t]{2}{*}{ FRF002 } & ROADSIDE & 977 & 56 & 16 & 13 & 19 \\
\hline & CONTROL & 977 & 61 & 27 & 26 & 36 \\
\hline MER002 & GOPHER & 1076 & 28 & 3 & 3 & 1 \\
\hline MEROO3 & CONTROL & 1103 & 198 & 44 & 49 & 75 \\
\hline YUF013 & $3 B \mathrm{GZ}$ & 1236 & 154 & 2 & 0 & 0 \\
\hline \multirow{2}{*}{ YUF001 } & $\mathrm{V} 2$ & 1237 & 375 & 39 & 17 & 36 \\
\hline & V3 & 1237 & 335 & 29 & 14 & 32 \\
\hline YUF012 & 3B CONTROL & 1239 & 232 & 22 & 11 & 28 \\
\hline YUF011 & 3B SCRAPED & 1239 & 0 & 0 & 0 & 0 \\
\hline YUF010 & T1 CONTROL & 1267 & 508 & 25 & 12 & 24 \\
\hline XUF009 & $\mathrm{T} 1 \mathrm{GZ}$ & 1279 & 42 & 1 & 0 & 1 \\
\hline YUF016 & SEDAN $1000^{\circ}$ & 1318 & 108 & 1 & 0 & 0 \\
\hline YUF017 & SEDAN $3000^{\circ}$ & 1327 & 169 & 10 & 4 & 12 \\
\hline YUF018 & SEDAN $5000^{\prime}$ & 1335 & 109 & 22 & 12 & 57 \\
\hline PAM001 & V1 & 1923 & 682 & 50 & 13 & 33 \\
\hline RAM001 & V1 & 2283 & 928 & 68 & 69 & 241 \\
\hline
\end{tabular}




\section{DISCUSSION}

Overall these data suggest the year 1988 was one of stasis for the established perennial plants on the NTS. The dominant shrub species neither grew significantly nor shrank significantly. Herbaceous perennials and bunchgrasses declined somewhat in numbers and sizes, and a young population of Hymenoclea salsola decreased in numbers and sizes except on disturbed areas.

Data from disturbed areas showed some patterns. The three sampled areas affected by blasts from nuclear tests (T1, T3, and Sedan) had sparse populations of bunchgrasses (Oryzopsis hymenoides, Sitanion jubatum, and Stipa speciosa), with some invasion of Hymenoclea salsola at Sedan and T1. The reduced cover on these locations after several decades was not a reflection of an inability of plants to grow, but rather an indication of the desert habitat, in which episodes of germination and establishment are rare. At Sedan, the more advanced successional stage of the blast area outside the area of subsoil deposits from the crater suggested an inhibition of seedling establishment by those deposits. The area of deposits was invaded shortly after the blast by Salsola paulsenii (Russian thistle) (Martin 1963; Hill unpublished data), which was dominant until the area was invaded by bunchgrasses during the 1970s (Hunter et al. 1987). Initial stages in vegetative recovery from these blasts were reported earlier (Shields et al. 1963; Beatley 1966; Rickard and Sauer 1982).

The gopher-denuded area sampled in Mercury Valley differed somewhat from the blast areas and also burned areas (Hunter and Medica 1989) in that the vegetation was dominated by the semi-shrub Stanleya pinnata, and the bunchgrasses were largely absent. The obvious explanation is that these areas are heavily grazed by gophers and other rodents and lagomorphs (Hunter et al. 1980), and S. pinnata is resistant to grazing (Hunter 1987).

The monitoring of the roadside habitat was an initial effort that did not adequately describe the interaction between road and desert vegetation. The location picked was on an abandoned section of paved road which had not been maintained (shoulders scraped) for about 20 years, allowing colonization by shrubs, which were young and vigorous. However, driving along the road for some distance showed that the species established on the road verge differed considerably at different locations. It was not possible to make generalizations about the vegetation along this road, because the perennial plant species and densities were too varied. The site sampled must be considered a specific site at which relative growth and species composition may be followed through time, but we cannot say at this time that it is representative of other roadside sites. A study was published by Johnson et al. (1975) which showed increases in both numbers of shrubs and cover associated with roadsides in the Mojave Desert. Our study site had lower cover near the road, a result of past scraping and road maintenance. The difference is probably the result of placement of the transects - we placed our two-meter wide transect immediately adjacent to the asphalt pavement, while 
Johnson et al. apparently adjusted the width of their transects to cover the observably larger plants adjacent to the scraped area. A proper assessment of the roadside effect would require a different sampling design repeated at more locations, which was beyond the scope of the present study.

Some of the rather simple changes in field technique used for the BECAMP studies resulted in significant changes in analytical procedures. In the past a representative area was sampled $\left(100 \mathrm{~m}^{2}\right)$ and several replicates could be used to indicate reliability of the measurements (Hunter and Medica 1989). An analysis of 1987 data (R. O. Gilbert, personal communication) showed that for the most common species five transects would allow measurement of densities to within about $35 \%$ of the true value. For intermediate and rare species the densities could be estimated within roughly $\pm 50 \%$ and $\pm 110 \%$, respectively. To get reliable estimates within $\pm 10 \%$ of density, cover, or biomass for any given species would not be possible with fewer than 70 transects. (However, total plant density could be estimated within $10 \%$, cover within about $\pm 20 \%$, and biomass within about $\pm 25 \%$ using five transects.) The amount of work involved in accurately determining these parameters for individual species is therefore not practical, and statistical comparisons between different sites and times cannot be practically made. This is largely a result of a high natural variability in these vegetative parameters, rather than inaccuracies in the technique.

Repeated sampling of the same transect, however, allowed remeasurement of individual shrubs to determine growth rates (Tables 6.10 and 6.12), establishment and survivorship. The data apply only to the measured sites, and therefore it is necessary to carefully mark the transects used so that they may be repeatedly sampled. Analyses of the 1987-1988 data (Tables 6.4-6.8) showed deficiencies occurred in the matching of plants from year to year, and these deficiencies need to be addressed and corrected in the future. The poor matching did not prevent measurement of rather small changes in the shrub sizes (Table 6.12), but did make it difficult to interpret small changes in numbers of plants. That information is important to documentation of death and establishment of shrubs.

One important measurement parameter which caused problems with matching plants was defining an individual when more than one shoot emerged from the ground. Many of the dominant species (Larrea tridentata, Ephedra nevadensis, Coleogyne ramosissima, Lycium andersonii, Yucca brevifolia, and Yucca schidigera) can produce either multiple stems or rhizomatous shoots. This problem can be addressed in several ways (Ebert and Ebert 1989), including training and "definition", but cannot be absolutely eliminated.

An addition to the data presented for 1988 is the total volume for a species on a transect. This allows a different interpretation of dominance from that provided by the traditional measurement of total cover. Total volume utilizes the information available on plant height, and in some locations there were significant differences in height among the species. In 
particular, Larrea tridentata dominance was better estimated, because it was generally the tallest species in its communities (Tables 6.4-6.6, 6.13 and 6.15).

The ratios of live to dead shrubs were generally greater than 6 . This suggests either that shrub life spans are much longer than the decades required for their wood to decay, or that their populations were young and not at equilibrium. Lifespans and decay rates are currently unknown, but future monitoring at these locations should provide estimates of both those factors.

In the future these sites will be monitored at three to five-year intervals. Techniques will be refined to improve the ability to match plants from year to year, and new sites will be established. They will be permanently marked to allow repeat sampling over long intervals. 


\section{REFERENCES}

Allred, D. M., D E. Beck, and C. D. Jorgensen. 1963. Close-in effects of an underground nuclear detonation on small mammals and selected invertebrates. Report PNE-226p, U.S. Atomic Energy Commission, Available from NTIS, U.S. Dept. of Commerce, Springfield, VA 22161.

Beatley, J. C. 1966. Winter annual vegetation following a nuclear detonation in the northern Mojave Desert (Nevada Test Site). Radiation Botany 6:69-82.

1976. Vascular plants of the Nevada Test Site and Central-southern Nevada: ecologic and geographic distributions. Energy Res. Devel. Admin. Report TID-26881. NTIS, U.S. Dept. of Commerce, Springfield, VA 22161. 308pp.

1979. Shrub and tree data for plant associations across the Mojave/Great Basin Desert transition of the Nevada Test Site, 1963-1975. Report DOE/EV/2307-15, U.S. Dept. of Energy. N IS, U.S. Dept. of Commerce, 5285 Port Royal Road, Springfield, VA 22161.

Ebert, T. A. and C. A. Ebert. 1989. A method for studying vegetation dynamics when there are no obvious individuals - virtual-population analysis applied to the tundra shrub Betula nana. L. Vegetatio 85:33-44.

Erickson, R. O. 1976. Modelling of plant growth. Annual Review of Plant Physiology 27:407434.

Hoaglin, D. C., F. Mosteller, and J. W. Tukey. 1983. Understanding Robust and Exploratory Data Analysis. New York, NY. John Wiley \& Sons.

Hunter, R. B. 1987. Jackrabbit-shrub interactions in the Mojave Desert. Proceedings, Symposium on Plant-Herbivore Interactions. USDA Forest Service Gen. Tech. Rep. INT222:88-92.

1989. Competition between adult and seedling shrubs of Ambrosia dumosa in the Mojave Desert, Nevada. Great Basin Naturalist 49:79-84.

and P. A. Medica. 1989. Status of the flora and fauna on the Nevada Test Site: results of continuing basic environmental research January through December 1987. Report DOE/NV/10630-2. 103pp. Available from NTIS.

Hunter, R. B., E. M. Romney, and A. Wallace. 1980. Rodent-denuded areas of the northern Mojave Desert. Great Basin Naturalist Memoirs 4:208-211. 
1987. Revegetation on disturbed desert land at NUWAX and Sedan. Pp. 79-97 in

Dynamics of transuranics and other radionuclides in natural environments, W. A. Howard and R. G. Fuller, (Eds.), U.S. Dept. of Energy Report NVO-272. Available from NTIS, U.S. Department of Commerce, Springfield, VA 22161.

Johnson, H. B., F. C. Vasek, and T. Yonkers. 1975. Productivity, diversity and stability relationships in Mojave Desert roadside vegetation. Bulletin of the Torrey Botanical Club 102:106-115.

Jorgensen, C. D. and C. L. Hayward. 1965. Mammals of the Nevada Test Site. Brigham Young Univ. Sci. Bull, Biol. Ser. 6(3). 81pp.

Kartesz, J. T. and R. Kartesz. 1980. A synonymized checklist of the vascular flora of the United States, Canada, and Greenland. Univ. North Carolina Press, Chapel Hill, NC. 500pp.

Lathrop, E. W. and E. F. Archbold. 1980. Plant response to Los Argeles Aqueduct construction in the Mojave Desert. Environmental Management 4:137-148.

Martin W. E. 1963. Close-in effects of an underground nuclear detonation on vegetation. 1. Immediate effects of cratering, throw-out, and blast. USAEC Report PNE-228p.

Munz, P. A. 1974. A Flora of Southern California. Univ. Calif. Press, Berkeley, CA 94720. 1086pp.

Pearson, L. C. 1975. Effects of temperature and moisture on productivity of Oryzopsis hymenoides. US/IBP Desert Biome Res. Memo. 75-6. Utah State Univ., Logan 84322. $16 \mathrm{pp}$.

Rickard, W. H., and R. H. Sauer. 1982. Self-revegetation of disturbed ground in the deserts of Nevada and Washington. Northwest Science 56(1):41-47.

Romney, E. M., V. Q. Hale, A. Wallace, O. R. Lunt, J. D. Childress, H. Kaaz, G. V. Alexander, J. E. Kinnear, and T. L. Ackerman. 1973. Some characteristics of soil and perennial vegetation in northern Mojave Desert areas of the Nevada Test Site. Report UCLA \#12916, TID-4500, available from NTIS, U.S. Dept. of Commerce, Springfield, VA 22151. 340 pp.

Shields, L. M., P. V. Wells and W. H. Rickard. 1963. Vegetational recovery on atomic target areas in Nevada. Ecology 44(4):697-705.

Sternberg, L. 1976. Growth forms of Larrea tridentata. Madroño 23(8): 405-417. 
Tanner, W. W., and J. M. Hopkin. 1972. Ecology of Sceloporus occidentalis longipes Baird and Uta stansburiana Baird and Girard on Rainier Mesa, Nevada Test Site, Nye County, Nevada. Brigham Young Univ. Sci. Bull., Biol. Ser. 15(4). 39pp.

Tausch, R. J., and P. T. Tueller. 1990. Foliage biomass and cover relationships between tree-dominated and shrub-dominated communities in pinyon-juniper woodlands. Great Basin Naturalist 50:121-134.

Van, T. R., and W. G. Spaulding. 1979. Development of vegetation and climate in the southwestern United States. Science 204:701-710.

Webb, R. H., and H. G. Wilshire. 1980. Recovery of soils and vegetation in a Mojave Desert ghost town. Journal of Arid Environments. 3:291-303.

Welsh, S. L., N. D. Atwood, S. Goodrich, and L. C. Higgins, editors. 1987. A Utah Flora. Great Basin Naturalist Memoirs 9. 894pp.

West, N. E., and C. Gunn. 1974. Phenology, productivity and nutrient dynamics of some cool desert shrubs. US/IBP Desert Biome Res. Memo. 74-7. Utah State Univ., Logan 84322. $6 \mathrm{pp}$. 
1.4 and 1.5), but the ability to use statistical tests on the numerous individuals allowed a better interpretation of changes in size. It should be noted that to show that plants neither grew nor shrank over a one-year period may be a significant observation, rather than an indication of measurement error. 
Thomas I. Ackexman

1515 Alice Street

No. 16

Oakland, California 94612 (1)

Lynn R. Anspaugh, Division Leader

Environmental Sciences Division

Lawrence Iivermore National Laboratory

I 453

P. O. Box 5507

Livermore, California 94550 (2)

BECAMP Library

$M / S 740$ (5)

Arden E. Bicker, Assistant Manager

Envixonment, Safety and Health

$\mathrm{M} / \mathrm{S} 765$ (1)

Frank E. Bingham, Manager

Environmental Compliance office

$M / S 711$ (1)

Brigham Young University Library

Exchange Section

Provo, Utah 84601

Charles D. Canham

Institute of Ecosystem studies

The New York Botanical Garden

Mary Elagler Gary Arboretum

Box AB

Millbrook, New York 12545 (1)

Dr. Donald R. Clark

Section of Environmental. Studies

Patuxent Wildife Research Center

Laurel, Maryland 20810 (2)

John Donaldson, Regional Manager

Nevada Department of Wildiife

Region III

State Mailroom Complex

Las Vegas, Nevada 89158

Dr. Charles L. Douglas

Department of Biological sciences

University of Nevada Las Vegas

4505 S. Maryland Parkway

Las Vegas, Nevada 89154 (1) 


\section{DISIRIBUTION ISST (continued)}

Donald R. Elle, Director

Environmental Protection Division DOE Nevada Field Office

P.O. Box $98518 \mathrm{M} / \mathrm{S} 505$

Las Vegas, Nevada 89193-8518

Dr. Edward H. Essington

Los Alamos National Laboratory

Environmental Science Group

HSE 12

Los Alamos, New Mexico 87545

Robert E. Friedrichs

DOE Nevada Field Office

P.O. Box $98518 \mathrm{M} / \mathrm{S} 505$

Las Vegas, Nevada 89193-8518

Bert Friesen

Raytheon Services Nevada

P.O. Box $95487 \mathrm{M} / \mathrm{S} 605$

Las Vegas, Nevada 89193-5487

Robert Furlow

DOE Nevada Field Office

P. O. Box $98518 \mathrm{M} / \mathrm{S} 505$

Las Vegas, Nevada 89193-8518

Dr. Thomas H. Fritts

U. S. Fish and Wildlife Service

National Museum of Natural History

Smithsonian Institution

Washingt on DC 20560 (1)

Dr. Whitfield Gibbons

Savannah River Ecology Lab

Drawer $\mathrm{E}$

Aiken, South Carolina 29801

Dr. Ri.chard 0. Gilbert

Battelle

Pacific Northwest Laboratories

P. O. Box 999

Richland, washington 99352

Ron Green

EG\&G/EM

P. O. Box $1912 \mathrm{M} / \mathrm{S} \mathrm{V}-01$

Las Vegas, Nevada 89125 


\section{DISTRIBUTIOA IIST (continued)}

Paul Greger

UCLA

P.O. Box $495 \mathrm{M} / \mathrm{S} 740$

Mercury, Nevada 89023

Harold Grey

EG\&G/EM

P. O. Box $1912 \mathrm{M} / \mathrm{S} \mathrm{V}-01$

Las Vegas, Nevada 89125

John B. Hall

DOE Nevada Field office

P. O. Box $98518 \mathrm{M} / \mathrm{S} 505$

Ilas Vegas, Nevada 89193-8518 (2)

Dr. K. T. Harper

Department of Botany

Brigham Young University

Provo, Utah 84602 (1)

Dr. Howard Hawthorne

REECO

P. O. Box $98521 \mathrm{M} / \mathrm{S} 708$

Las Vegas, Nevada 89193-8521

Dr. Richard B. Hunter

REECO

P. O. BOX $98521 \mathrm{M} / \mathrm{S} 740$

Las Vegas, Nevada 89193-8521

Dr. Clive D. Jorgensen

Brigham Young University

Department of Zoology

574 Widtsoe Building

Provo, Utah 84602 (1)

Michael Mc Laurin

EG\&G/EM

P. O. Box $1912 \mathrm{M} / \mathrm{S} \mathrm{V}-01$

Las Vegas, Nevada 89125

Philip A. Medica

P. O. Box 26569

Las Vegas, Nevada 89126

Stephen A. Mellington

DOE Nevada Field office

P. O. Box 98518

Las Vegas, Nevada 89193-8518 
Dr. Michael J. O'Farrell

$2912 \mathrm{~N}$. Jones Boulevard

Las Vegas, Nevada 89108

Dr. Thomas P. O'Farrell

511 Avenue $\mathrm{H}$

Boulder City, Nevada 89108

Kent Ostier

EG\&G/EM

P. O. Box $1912 \mathrm{M} / \mathrm{S} \mathrm{V}-01$

Las Vegas, Nevada 89125 (1)

Scott E. Patton

Lawrence Livermore National Laboratory

$\mathrm{L}-453$

P. O. Box 5507

Livermore, California 94550

Dr. Ionnie C. Pippin

Desert Research Institute

Social Sciences Center

R. O. Box 60220

Reno, Nevada 89506

Daniel Rekestraw

EG\&G/EM

P. O. Box $1912 \mathrm{M} / \mathrm{S} \mathrm{V}-01$

Las Vegas, Nevada 89125

Kurt Rauthenstrauch

EG\&G/EM

P. O. Box $1912 \mathrm{M} / \mathrm{S} \mathrm{V}-01$

Las Vegas, Nevada 89125

David Rabrock, Head

Special Collections

UNLV Iibrary

4505 S. Maryland Parkway

Las Vegas, Nevada 89154

Dr. Evan M. Romney

$11074 \mathrm{~N}$. Wahoo Trail

Dunnelion, Florida 32630

Mary Saethre

UCI,A

P. O. Box $495 \mathrm{M} / \mathrm{S} 740$

Mercury, Nevada 89023 


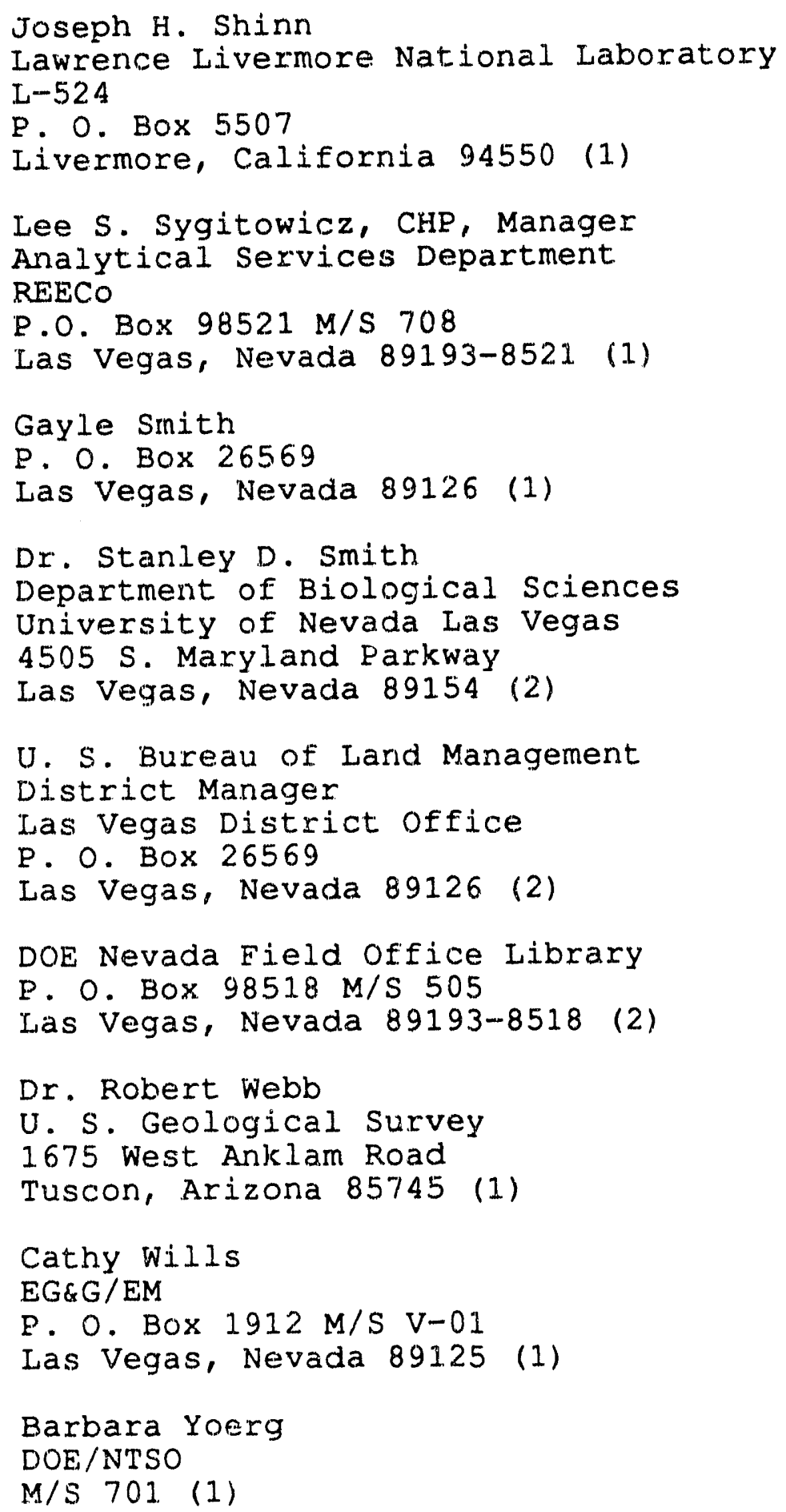



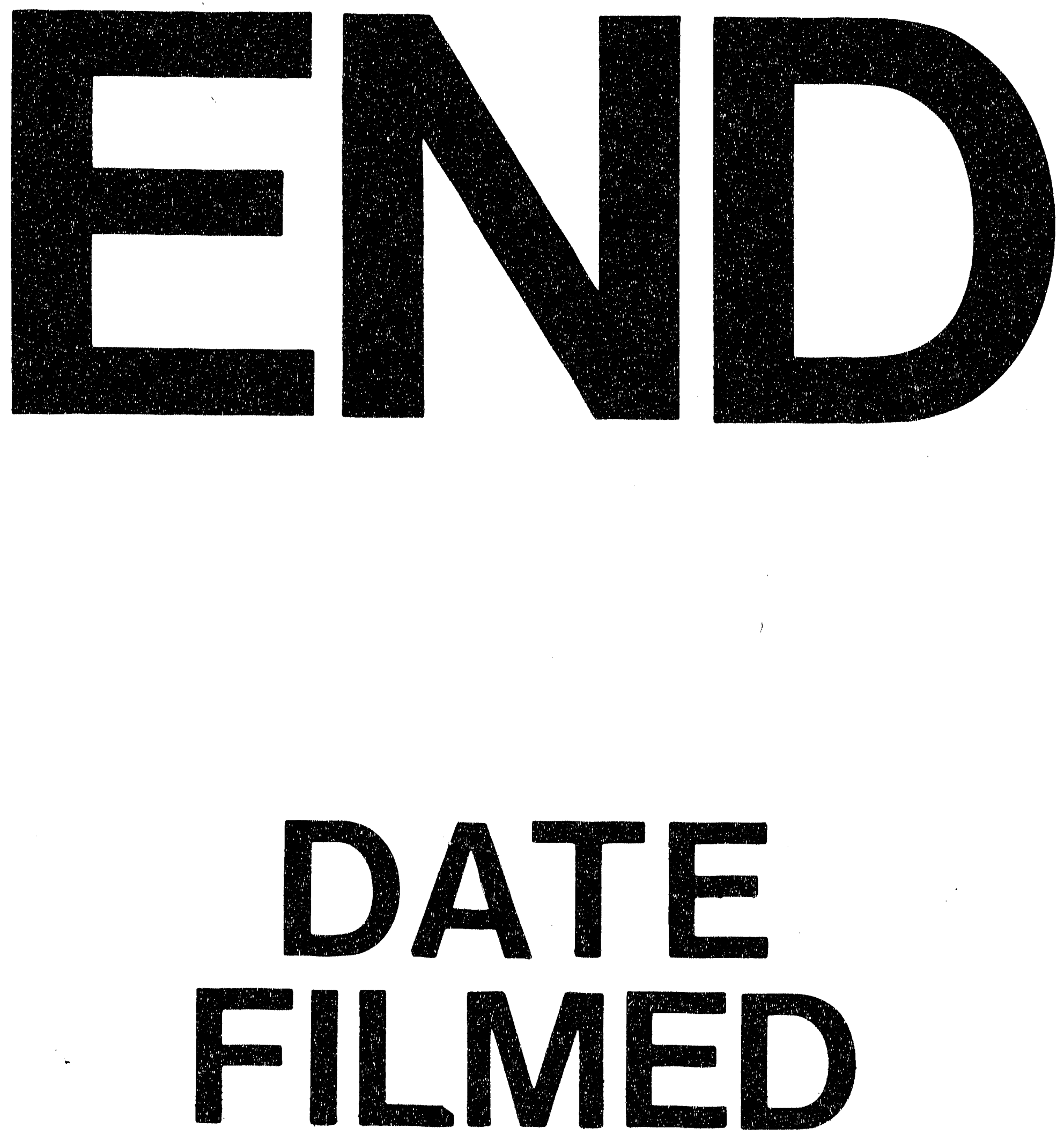

爱

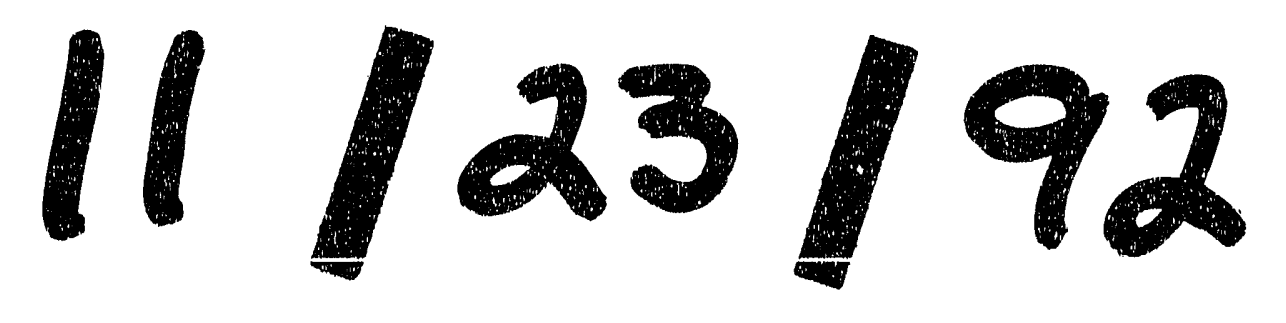


